\title{
NEW STRATEGIES FOR THE DETECTION OF FUSARIUM INFECTION AND MYCOTOXIN CONTAMINATION OF CEREALS AND MAIZE
}

\author{
Dissertation \\ zur Erlangung des Doktorgrades \\ der Fakultät für Agrarwissenschaften \\ der Georg-August-Universität Göttingen \\ vorgelegt von \\ Eva-Maria Becker (geb. Kuhl) \\ geboren in Marburg
}

Göttingen, im März 2013 
D 7

1. Referentin/Referent: Prof. Petr Karlovsky

2. Korreferentin/Korreferent: Prof. Stefan Schütz

Tag der mündlichen Prüfung: $\quad$ 14. Mai 2013 


\section{Contents}

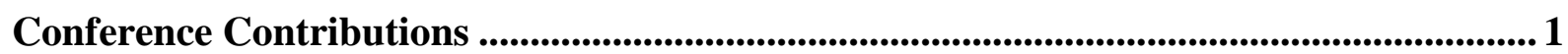

Project \& Funding ...................................................................................................................... 1

Patent Application .................................................................................................................................. 2

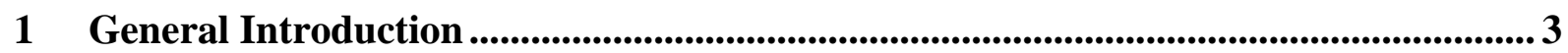

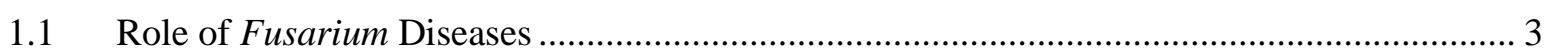

1.2 Symptoms and Epidemiology of Fusarium Diseases in Wheat and Maize ............................. 4

1.2.1 'Fusarium Head Blight' (FHB) of Wheat....................................................................... 4

1.2.2 'Gibberella Ear Rot' and 'Fusarium Ear Rot' of Maize ..................................................... 4

1.2.3 Epidemiology of $F$. graminearum and $F$. verticillioides ............................................. 5

1.3 Important Secondary Metabolites in the Fusarium - Plant Pathosystem .................................. 6

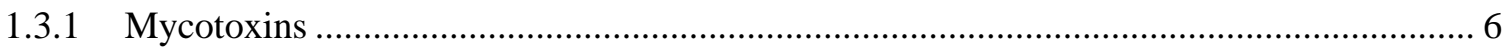

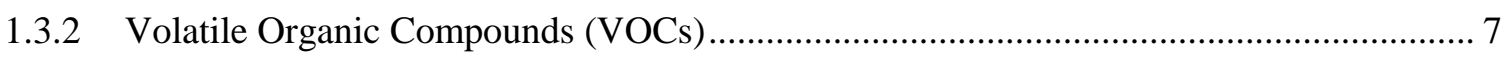

1.3.3 Other Secondary Metabolites Involved in Plant Defense Reactions ............................... 11

1.4 A Fungal Antagonist of Fusarium spp. and its Potential Use for the Specific Detection of

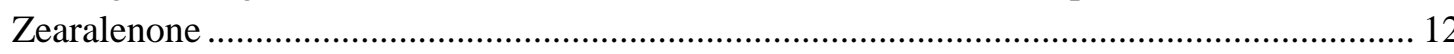

1.4.1 The Mycoparasite Gliocladium roseum, its Features and its Use as a Biocontrol Agent Against Phytopathogenic Fungi ............................................................................. 12

1.4.2 Bioassays for the Identification of Estrogenic Zearalenone ............................................ 14

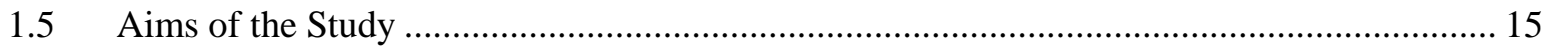

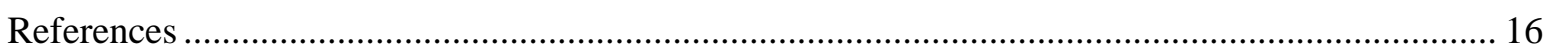

2 Volatile Markers for the Infection of Maize (Zea mays L.) Ears with Fusarium spp...

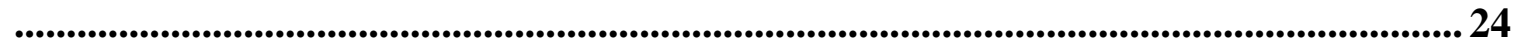

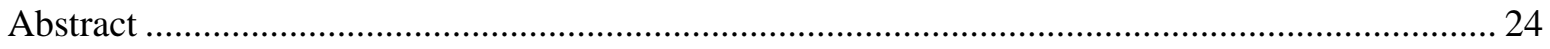

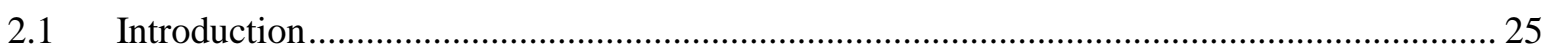

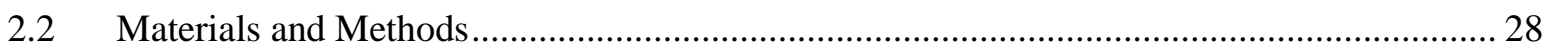

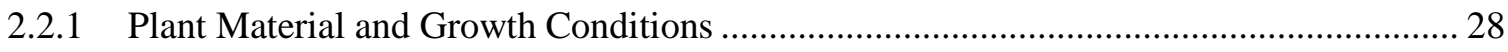


2.2.2 Fungal Strains 28

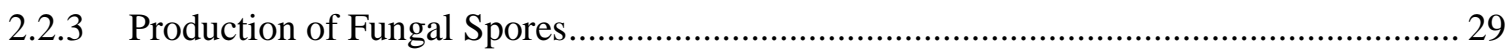

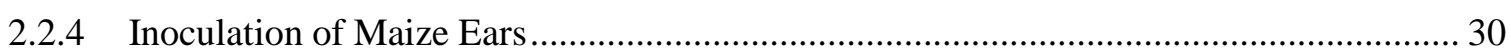

2.2.5 Handling of Plant Material for Chemical and Molecular Analysis.................................... 31

2.2.6 Collection of Volatiles Using Static Procedure …........................................................ 33

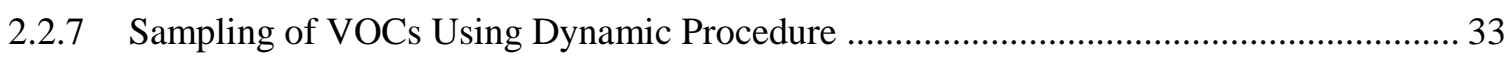

2.2.8 Gas Chromatography (GC) Conditions for Volatile Profiling .......................................... 35

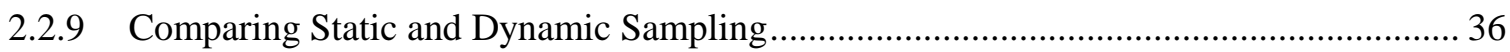

2.2.10 In vitro Assay with Fusarium Cultures on Autoclaved Maize and Rice Kernels........ 36

2.2.11 Effect of VOCs from Infected Ear Material on Fungal Growth .................................. 37

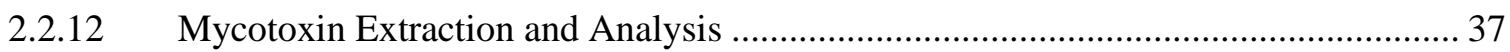

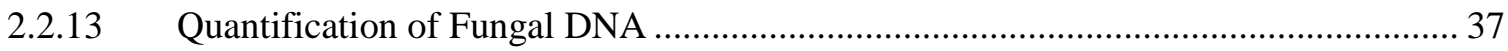

2.2.14 Analysis of Terpene Synthase Genes tps6/11 …........................................................ 39

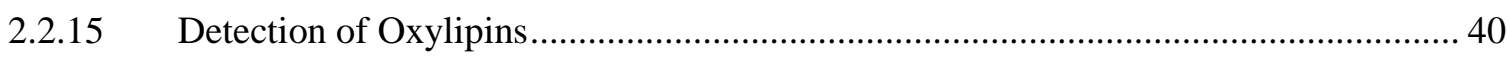

2.2.16 Extraction and Analysis of Zealexins and Jasmonic Acid (JA)................................. 40

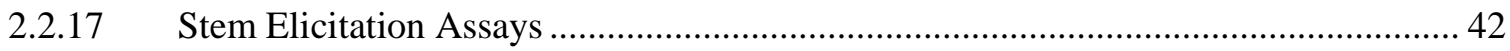

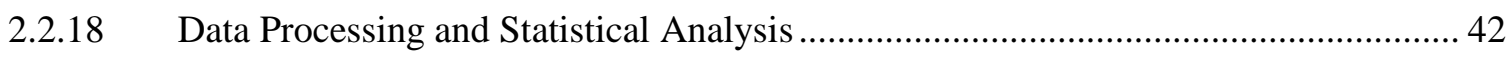

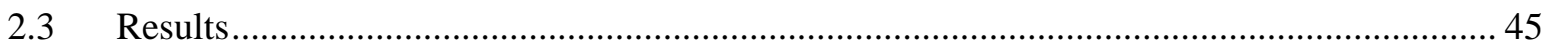

2.3.1 Optimization of a Specific Real-Time PCR Assay for F. subglutinans …........................ 45

2.3.2 Comparison of Dynamic and Static Sampling Strategies for the Collection of VOCs..... 46

2.3.3 Set of Volatile Biomarkers for Fusarium Infection of Maize Ears by SPME/GC-MS

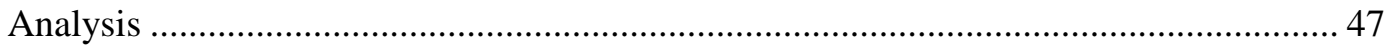

2.3.4 Volatiles Found in Mixed Infection Treatments of Hybrid Maize by SPME/GC-MS

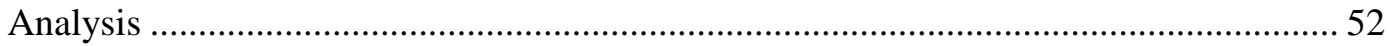

2.3.5 Temporal Release of Volatile Compounds During Pathogenesis (SPME/GC-MS Analysis)

2.3.6 Confirmed VOC Biomarkers by Non-Destructive Sampling (OLS) and Range of

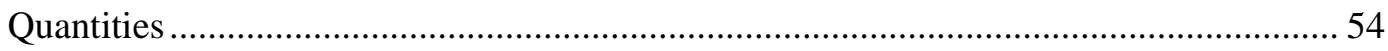

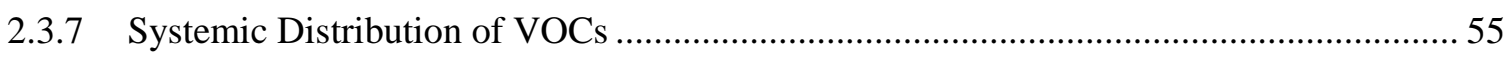

2.3.8 Volatile Spectra of in vitro Cultures on Maize and Rice and Effect of Infected Maize Volatiles on Fungal Growth..... 
2.3.9 Fungal Biomass, Disease Severities and Mycotoxin Production in Infected Hybrid Maize

2.3.10 Transcription of Terpene Synthase Genes in Infected Dwarf Maize ............................ 58

2.3.11 Oxylipins in Fusarium Infected Hybrid Maize Ears..................................................... 59

2.3.12 Induction of Volatiles Upon Stem Treatment of Dwarf Maize with Jasmonic Acid and

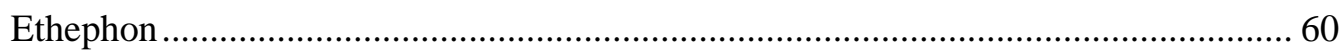

2.3.13 Zealexins and Phytohormones in Fusarium Infected Dwarf Maize Ears .................... 61

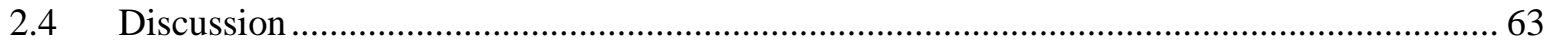

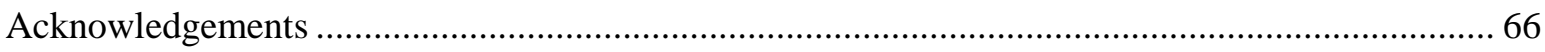

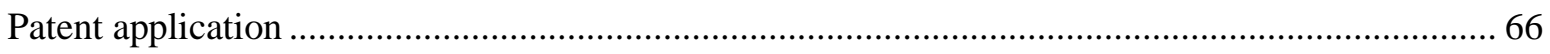

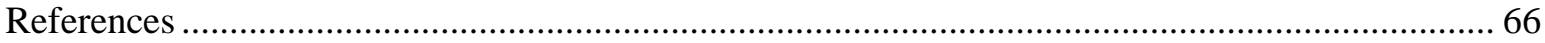

3 Volatile Biomarkers for the Fusarium Infection of Wheat (Triticum aestivum L.)

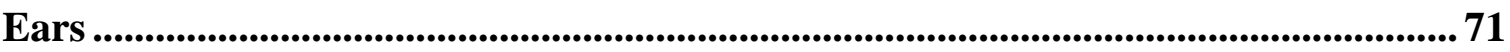

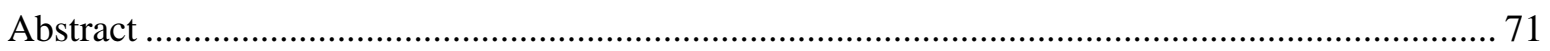

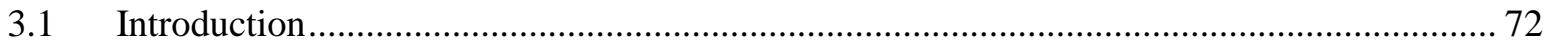

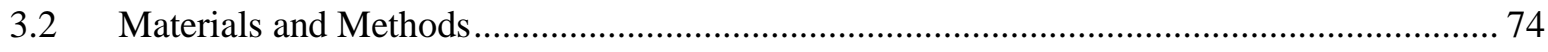

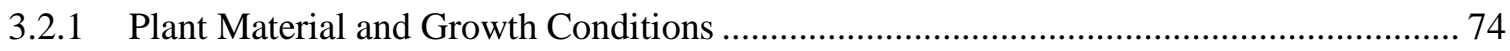



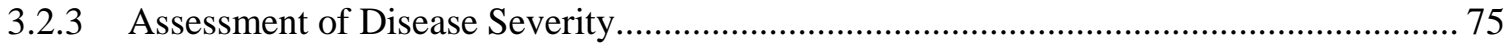

3.2.4 Sample Preparation and Collection of Volatile Molecules ................................................ 76

3.2.5 In vitro Assay with Autoclaved Wheat and Rice Kernels ................................................. 77

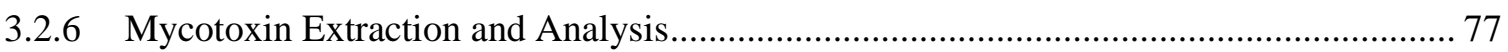

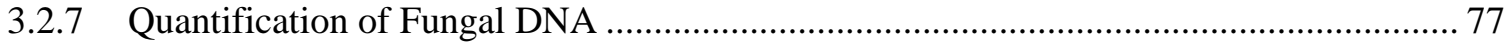

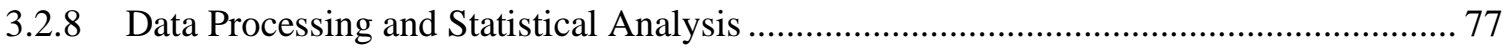

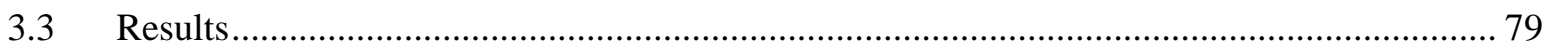

3.3.1 Selected Volatile Markers for the Fusarium Infection of Wheat..................................... 79

3.3.2 Volatile Terpenoids Released Upon Moderate Infection with $F$. avenaceum and $F$. poae ..

3.3.3 Time Series - Temporal Release of Volatile Compounds from Fusarium Infected Wheat Ears . 


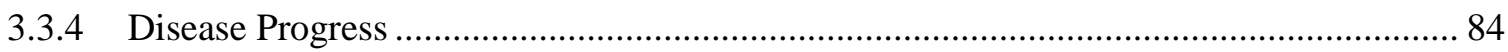

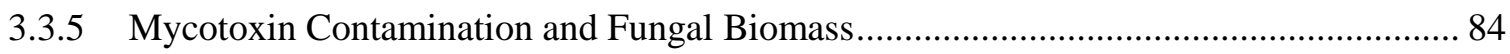

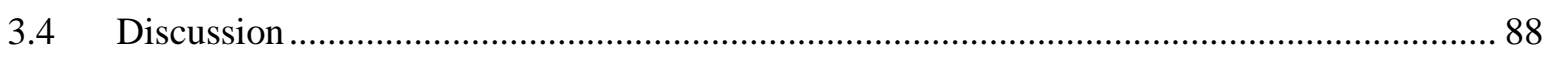

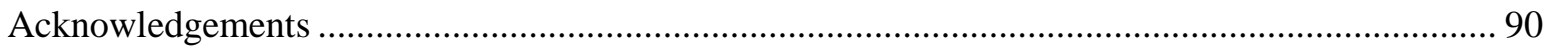

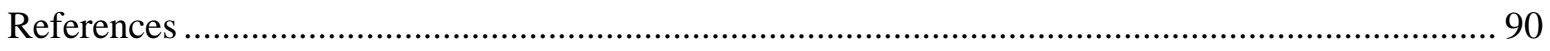

\section{A Bioassay for Zearalenone (ZEN) Based on the Mycoparasite Gliocladium roseum..}

Abstract 92

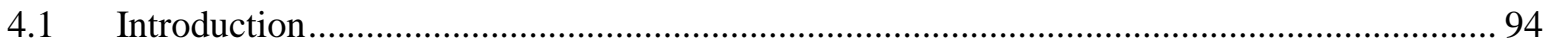



4.2.1 Recombinant Gliocladium roseum Strain and Spore Production ..................................... 97

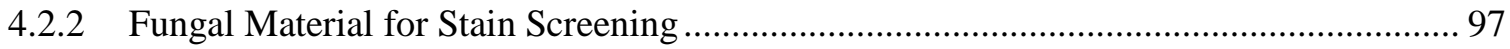

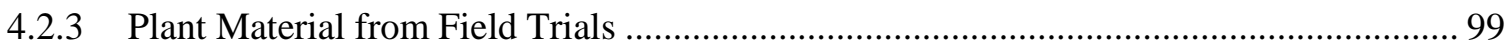

4.2.4 General Setup of the G. roseum Bioassay and Measurement Procedure .......................... 99

4.2.5 Assessment of Bioassay Kinetics and Detection Limits in General Procedure .............. 100

4.2.6 Evaluation of Assay Specifity to ZEN, its Reductive Metabolites and Other Estrogens 101

4.2.7 Alternative Setup - A Procedure with Direct Exposure of G. roseum Spores to ZEN... 101

4.2.8 General Procedure with Solvent Extracts of Maize Field Samples After Clean-up with

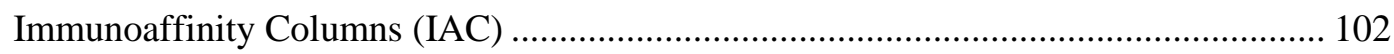

4.2.9 General Procedure with Extracts of Maize Field Samples After Conventional Solid-Phase

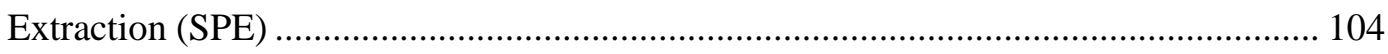

4.2.10 Calculation of Matrix Correction Factors for IAC and Conventional SPE Clean-up of Maize Samples in General Procedure

4.2.11 Bioassay with Extracts from Field Samples Employing Conventional SPE Clean-up and

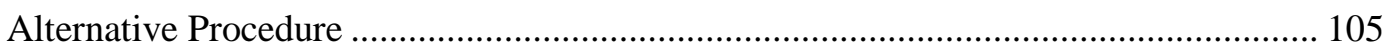

4.2.12 Data Processing and Calculation of ZEN Concentration in Field Samples ................ 105

4.2.13 Cultivation of Fungal Strains and Extraction of ZEN for Strain Screening .............. 106

4.2.14 Plate Layout for Fungal Strain Screening ............................................................. 106

4.2.15 Use of Defatted Crude Extracts from in vitro Cultures for Fungal Strain Screening 107

4.2.16 Data Evaluation for Fungal Strain Screening ....................................................... 107 


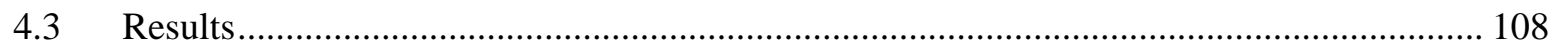

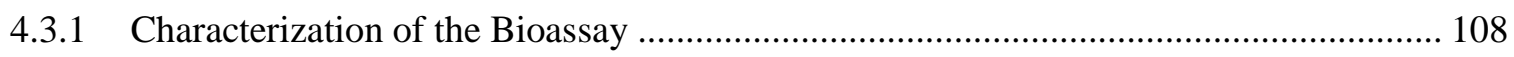

4.3.1.1 Response of G. roseum zes2::gfp Strain to ZEN and Other Estrogens .................108

4.3.1.2 Assay Kinetics in General Procedure with Pre-Culturing of G. roseum ................109

4.3.1.3 Assay Kinetics in Alternative Procedure with Direct Exposure of G. roseum

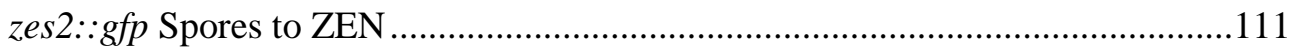

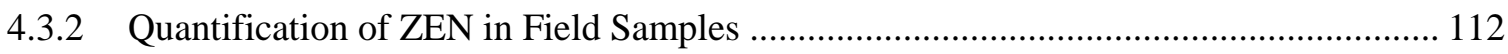

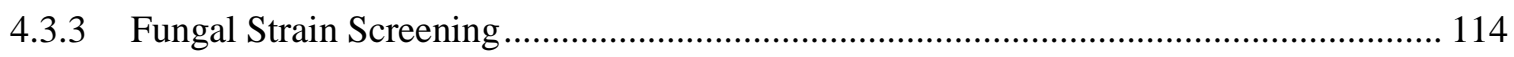

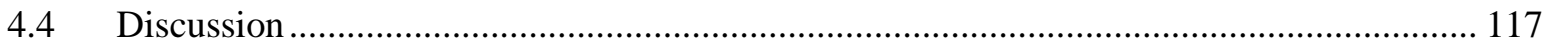

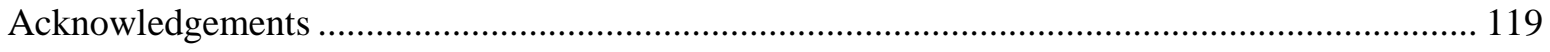

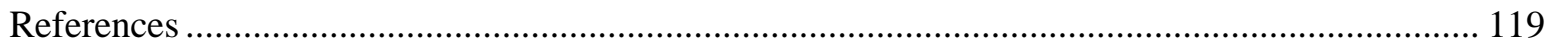

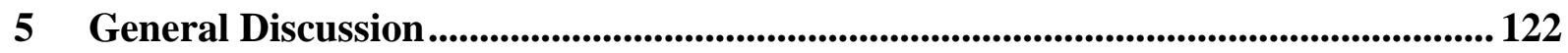

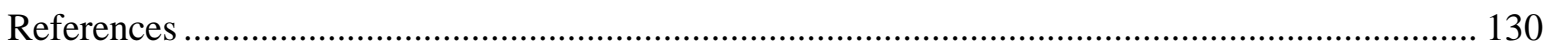

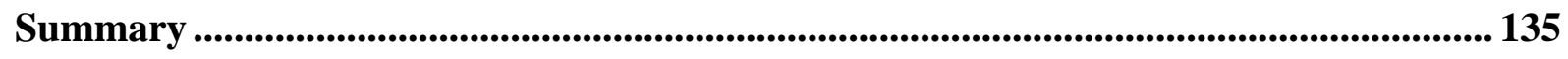

Zusammenfassung ................................................................................................................................. 137

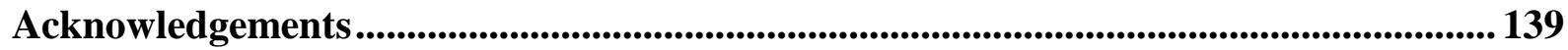

Curriculum vitae ................................................................................................................................... 141

Eidesstattliche Erklärungen .......................................................................................................... 142 


\section{Conference Contributions}

Eva-Maria Kuhl, C. Goebel, I. Feußner and P. Karlovsky (2010), Mycotoxin production in maize inoculated with Fusarium graminearum, Fusarium verticillioides and Fusarium subglutinans, $32^{\text {nd }}$ Mycotoxin Workshop, Lyngby, Denmark (poster presentation)

Eva-Maria Becker, J. Utermark and P. Karlovsky (2012), Bioassay based on Gliocladium roseum zes2-GFP strain responds to zearalenone but not other estrogens, $34^{\text {nd }}$ Mycotoxin workshop, Braunschweig, Germany (poster presentation)

Eva-Maria Becker, R. Splivallo and P. Karlovsky (2012), Early prediction of mycotoxin contamination in maize based on detection of volatile organic compounds, $34^{\text {nd }}$ Mycotoxin workshop, Braunschweig, Germany (oral presentation)

Eva-Maria Becker, R. Splivallo and P. Karlovsky (2012), Changes in the composition of volatile organic compounds (VOCs) of maize cobs infected with mycotoxin producing Fusarium spp., 58. Deutsche Pflanzenschutztagung, Braunschweig, Germany (poster presentation)

\section{Project \& Funding}

The work on volatile biomarkers for the infection of wheat and maize with toxigenic Fusarium species was kindly funded by the Federal Ministry of Education and Research and supported by the German Aerospace Centre (DLR). Further information on the project 'MykoSensExpert' is available at http://www.proplant.de/MykoSensExpert/index.html. 


\section{Patent Application}

A patent on early volatile biomarkers for Fusarium infection in maize was filed in March 2012 (German title 'Volatile Biomarker fuer die Detektion von Mykotoxin produzierenden pilzlichen Pathogenen bei Maispflanzen', DE 101012204237.7). The University of Goettingen (Germany) is the patent holder. Inventors are Eva-Maria Becker, Dr. Richard Splivallo and Prof. Dr. Petr Karlovsky. The application process was kindly guided by Dr. Carlos Guentner (MbM Science Bridge, University of Goettingen, Germany). 


\section{General Introduction}

\subsection{Role of Fusarium Diseases}

The widespread genus Fusarium belongs to the phylum Ascomycota (tubular fungi), which represents about $30 \%$ of all known fungal species. The genus was first described by Link (1809). Wollenweber \& Reinking (1935) established the basis for a taxonomic system, although nomenclatural aspects of Fusarium were always discussed controversially (Summerell et al., 2010; Leslie \& Bowden, 2008; Samson \& Gams, 1984). In this context, there are morphological, biological and phylogenetic species concepts available (Taylor et al., 2000; Gerlach \& Nirenberg, 1982; Mayr, 1963). Anamorph and teleomorph genera were described and the teleomorph genus Gibberella (anamorph Fusarium) includes some of the most important species of plant pathogens (Summerell et al., 2010; Desjardins, 2003). Fusarium diseases occur worldwide on important agricultural and horticultural crops and lead to the reduction of yield, seed quality and contamination of food commodities with toxic secondary metabolites, so called mycotoxins (Osborne \& Stein, 2007; Windels, 2000). Fusarium spp. are of outstanding relevance for the production of maize (Zea mays L.) and wheat (Triticum aestivum L.) as well as other small-grain cereals (Logrieco et al., 2002; McMullen et al., 1997). As a consequence of increasing demands for energy use, animal food and human diet, the world maize production is increasing steadily (Shiferaw et al., 2011; Nuss \& Tanumihardjo, 2010; Oslaj et al., 2010). Most important maize producers in 2010 were USA with 316 million tons (37\%, 33 million hectares), China with 178 million tons (22\%, 33 million hectares) and Brazil with 56 million tons (7 \%, 13 million hectares). Entire world production of maize in 2010 was about 844 million tons on 162 million hectares (FAO, 2012). Because of its excellent nutrition quality, wheat flour is the main commodity for bakery products. Moreover, it is used as a substrate for malt and starch production and to lower extends for energy purposes and animal food (Entwistle et al. 1998, Faridi et al., 1989). Wheat production was about 654 million tons on 217 million hectares in 2010. Most important producers in 2010 were China (18\%), India (12\%) and USA (9\%). Wheat production increased over the last decades (FAO, 2012). 
From the phytopathological point of view, plant residues of the former crop, especially maize, seem to be a major source of inoculum (Maiorano et al., 2008; Parry et al., 1995). It is known that the increase of conservation tillage, as economically favoured treatment, facilitates Fusarium development and contamination with mycotoxins in the following crop (Blandino et al., 2010; Dill-Macky \& Jones, 2000). The increase of cultivation area, trends in post-harvest treatment and short intercropping phases between host plants lead to an intensification of the Fusarium problem (Edwards, 2004).

\subsection{Symptoms and Epidemiology of Fusarium Diseases in Wheat and Maize}

\subsection{1 'Fusarium Head Blight' (FHB) of Wheat}

Fusarium spp. play a serious role as pathogens in the worldwide production of wheat and other small-grain cereals (Bottalico \& Perrone, 2002). The symptom is described as Fusarium head blight (FHB). It occurs as darkened necrotic lesions at the bottom of the ear and typical tissue bleaching ('blight') of upper spikelets. Some infected spikelets may show a brown discolouration (Goswami \& Kistler, 2004). The disease is predominated by F. graminearum Schwabe, F. culmorum (W. G. Smith) Sacc. and F. avenaceum (Fr.) Sacc., but other Fusarium spp. might be associated (Parry et al., 1995).

\subsection{2 'Gibberella Ear Rot' and 'Fusarium Ear Rot' of Maize}

Fusarium infection of maize can be divided in two major characteristics, Giberella ear rot or 'red ear rot' and Fusarium ear rot or 'pink ear rot'. Red ear rot of maize is essentially caused by F. graminearum (Discolour section). Associated symptoms, like extensive red or pink mold, usually emerge from the tip of the ear and extend downwards (Munkvold, 2003). Other Fusarium spp., like F. culmorum and F. avenaceum, may be grouped with this disease (Logrieco et al., 2002). Pink ear rot is mainly caused by F. verticillioides (Sacc.) Nirenberg (syn. F. moniliforme, Liseola section) (Nirenberg \& O’Donnell, 1998). Typical symptoms occur on single kernels or a small group of kernels as white or pink mold. Associated species are F. proliferatum (Matsushima) Nirenberg and F. subglutinans (Wollenweber \& Reinking) P.E. Nelson, Toussoun \& Marasas (Logrieco et al., 2002). 


\subsubsection{Epidemiology of $F$. graminearum and $F$. verticillioides}

The main inducer of red ear rot and FHB is homothallic $F$. graminearum. The species bridges the time gap between host crops by development of chlamydospores on remaining plant residues (Nyvall, 1970). During favourable conditions, it produces perithecia (teleomorph Gibberella zeae). The released ascospores can be considered a main source of inoculum, but the release of macroconidia from sporodochia may also play a role (Paul et al., 2004). The propagules can be distributed by wind and water splashes (Parry et al., 1995). Considering red ear rot, the infection pathway through the silk channel of maturing maize ear is most important (Miller et al., 2007). Furthermore, the transmission of $F$. graminearum from seeds to seedlings of maize was described by Kabeere et al. (1997). Infection of wheat ears occurs during anthesis or shortly after. Optimal temperature for F. graminearum development is considered to be in the range of $15-25^{\circ} \mathrm{C}$. High moisture content, frequent rainfall and moderate temperature conditions facilitate fungal growth and production of mycotoxins (Osborne \& Stein, 2007, Gilbert \& Fernando, 2004, Doohan et al., 2003).

Pink ear rot is mainly caused by $F$. verticillioides. During time gaps between host crops, F. verticillioides produces thickened hyphae as survival structures. Under favourable conditions, it produces a large number of microconidia and macroconidia on plant residues. Those asexual spores can be considered a major source of airborne inoculum. Infection through silk channel, as described for $F$. graminearum, is assumed to be the most important pathway (Munkvold et al., 1997). The transmission of $F$. verticillioides from infected seeds to the maize ear, via colonization of the entire plant, has been discussed recently (MurilloWilliams \& Munkvold, 2008). In this context, a symptomless infection of vegetative plant organs and maize ears has been described (Bacon \& Hinton, 1996). Another infection pathway might be the transfer of inoculum by insects, such as European corn borers (Ostrinia nubilalis) or sap beetles (Coleoptera: Nitidulidae) (Parsons \& Munkvold, 2010; Schulthess et al., 2002). Even the attraction of insects by volatile compounds, released by F. verticillioides, was discussed (Bartelt \& Wicklow, 1999). Growth of F. verticillioides is facilitated by warm and dry conditions. A temperature range around $30{ }^{\circ} \mathrm{C}$ and even drought stress was described to be suitable for fungal development and production of mycotoxins (Munkvold, 2003; Reid et al., 1999; Marin et al., 1995). The related species F. proliferatum and F. subglutinans share most important infection characteristics. Nevertheless, they have not been extensively studied and key elements of their development need to be understood (Munkvold, 2003). 


\subsection{Important Secondary Metabolites in the Fusarium - Plant Pathosystem}

\subsubsection{Mycotoxins}

Mycotoxins are secondary metabolites of filamentous fungi with pharmacological activity. The metabolites are related to diseases of humans and vertebrates (Bennett \& Klich, 2003). Most important mycotoxins, associated with fusarioses, are trichothecenes, such as deoxynivalenol (DON, syn. vomitoxin) and nivalenol (NIV), but also fumonisins and zearalenone (Placinta et al., 1999).

Structurally, trichothecenes are based on an epoxide containing sesquiterpenoid skeleton. They can be divided by their macrocyclic and nonmacrocyclic structures. The group of nonmacrocyclic trichothecenes is, furthermore, subdivided in type A trichothecenes, such as T-2 and HT-2 toxin, and type B trichothecenes, including DON/ADON (3/15ADON as acetyled derivates) and NIV (Kimura et al, 2007; Bennett \& Klich 2003). The formation of type A trichothecenes is predominated by $F$. sporotrichioides, while type B trichothecenes are produced by $F$. graminearum and F. culmorum (Placinta et al., 1999). Previous investigations indicate a role of trichothecenes in the pathogenesis in plants (Langevin et al., 2004; Proctor et al., 2002; Proctor, 1995). DON is known to exert multiple toxic effects in humans and animals, such as inhibition of protein synthesis, immunosuppression, anorexia and weight loss, but has comparatively low acute toxic effects ( $\mathrm{LD}_{50 \text { (mouse) }} 78 \mathrm{mg} \mathrm{kg}^{-1}$ (oral), $49 \mathrm{mg} \mathrm{kg}^{-1}$ (intraperitoneal, i.p.)) (Rotter, 1996; Forsell et al., 1987). Lethal toxicity of NIV is described to be 10 times higher (Ueno, 1984). Thresholds for maximal DON concentration in agricultural commodities are established in the European Union. The DON threshold for unprocessed wheat and maize is fixed to $1.75 \mathrm{mg} \mathrm{kg}^{-1}$ (Commission Regulation (EC) no. 1126/2007) and the blending of highly contaminated and less contaminated batches is not permitted.

The occurrence of fumonisins in Fusarium infected maize and maize products plays an important role as health risk. Fumonisins are polar mycotoxins, mainly produced by $F$. verticillioides. They derive from the condensation of alanine into an acetate-derived precursor. At least 15 compounds belong to the group of fumonisins, but fumonisin B1 (FB1) is considered to be the most important compound (Bennett \& Klich, 2003). Fumonisins were first mentioned in context with leokoencephalomalacia in horses (Marasas et al., 1976) and are known to have cancer-promoting activities (Gelderblom et al., 1988). A treshold of 
$4.0 \mathrm{mg} \mathrm{kg}^{-1}$ for the concentration of fumonisins B1 and B2 in unprocessed maize is actually implemented by the European commission (Commission Regulation (EC) no. 1126/2007). Although fumonisins are phytotoxic, it is assumed that they are not required during pathogenesis on maize (Desjardins \& Plattner, 2000).

Zearalenone (ZEN), previously known as F-2 toxin, is a resorcyliyc acid lactone, produced through the polyketide pathway by Fusarium species. Most important producers are $F$. graminearum and F. culmorum. ZEN is known to have a relatively low toxicity $\left(\mathrm{LD}_{50 \text { (rat) }}>\right.$ $10000 \mathrm{mg} \mathrm{kg}^{-1}$ ), although its estrogenic properties play an important role in animal production, especially in the production of pigs (Zinedine et al., 2007). The mycotoxin, which was also described as phyto- or mycoestrogen, resembles $\beta$-estradiol and binds to estrogen receptors in mammalian cells. The estrogenic activity is increased by reduction of ZEN to a-zearalenol (Kuiper et al., 1998; Kuiper-Goodman et al., 1987). A formulation including $\alpha$-zearalanol (zeranol, Ralgro ${ }^{\circledR}$ ) is still used as an anabolic agent in animal production outside of the European Union (Thevis et al., 2011; Bennett \& Klich, 2003).

In the last decades, enzyme-linked immunosorbent assays (ELISA) were used for the detection of mycotoxins. Nowadays, liquid and gas chromatography coupled with mass spectrometry are the favoured methods (Placinta et al., 1999).

\subsubsection{Volatile Organic Compounds (VOCs)}

Volatile organic compounds (VOCs) are low-molecular carbons with a high vapour pressure. They belong to several chemical classes and can travel large distances in heterogeneous environments. Most organisms, i.e. plants and microbes, are able to release VOCs. However, each organism tends to produce a specific mixture of volatiles that might be characteristic of that organism under a given environmental and physiological condition (Holopainen \& Gershenzon, 2010; Kesselmeier \& Staudt, 1999).

Plant volatiles are released from the generative and vegetative parts and belong to the group of terpenes, non-terpene aliphatics, phenylpropanoids and benzenoids (Tholl et al., 2006). It is well known that plant VOCs are a major component of essential oils (Bakkali et al., 2008). They play a role in indirect plant defense mechanisms, such as the attraction of parasitoids after herbivory (Kessler \& Baldwin, 2001; Dicke et al., 1990; Turlings et al., 1990). Moreover, they are known to have antimicrobial and antifungal properties 
(Soliman \& Badeaa, 2002, Daferera et al., 2000, Baratta et al., 1998). Upon injury, a fast de-novo synthesis of $\mathrm{C}_{6}$ compounds, so called green leaf volatiles (GLVs), with defensive features was observed (Bate \& Rothstein, 1998). In contrast to other studies, Mérey et al. (2011) found no attraction of beneficial insects in maize fields when synthetic GLVs were released. The group observed a dose dependant attraction of herbivores by GLVs. In context with stress situations, the role of VOCs in communication between neighbouring plants, so called 'plant talk', was discussed intensively (Baldwin et al., 2006, Arimura et al., 2005, Dicke et al., 2003). Besides biotic stress situations, abiotic stress, like drought and high temperatures, can influence the volatile composition that is released by plants (Loreto \& Schnitzler, 2010, Gouinguene \& Turlings, 2002).

Filamentous fungi produce a number of characteristic volatiles on stored cereals, such as 1-octen-3-ol, 3-methyl-1-butanol and 3-octanone, but also terpenoid substances (Boerjesson et al., 1989; Kaminski et al., 1974). The volatiles may be part of infection and competition processes (Kramer \& Abraham, 2011; Linton \& Wright, 1993). Previous studies indicate the use of these fungal compounds for the evaluation of food quality in storage processes (Magan \& Evans, 2000).

In general, the sampling of VOCs is possible through either dynamic or static sampling. The dynamic system or loop-stripping system was first described by Grob (1973), who applied the method on water samples. In the case of open-loop-stripping (OLS), a stream of ambient air is pulled or respectively pushed by a pump through a sample covering vessel. Volatile molecules enrich selectively on an adsorbing matrix (i.e. carbon or Tenax ${ }^{\circledR}$ ), which is usually packed in a glass cartridge. This assembly is called the volatile trap. It is placed behind the sample container in the direction of a pulling pump. In contrary to a closed-loop system (CLSA), the incoming air is renewed continuously. A dynamic sampling takes typically several hours (Jelen et al. 1995). After this process, the volatiles can be released from the adsorbing matrix by solvent extraction or by thermal desorption. The dynamic sampling of volatiles was extensively used in the last decades. Each application requires a distinct optimization with respect to sample properties (Vichi et al., 2007; Dickschat et al., 2004; Bestmann et al., 1997; Buttery \& Ling, 1995; Donath \& Boland 1995).

In the static procedure of volatile collection the sample is placed in a glass vial that is sealed air tight. Volatiles are released from the sample by diffusion and can be trapped on an adsorbent material that is exposed in the gas phase around the sample, the so called headspace 
(HS). The most common procedure for static headspace collection is the solid-phase microextraction (SPME), which was first described by Arthur \& Pawliszyn (1990). The SPME technique is based on a fused silica fiber that is coated with a specific polymer, the stationary phase. Common polymers are polydimethyl-siloxane (PDMS), divinylbenzene (DVB) and Carboxen. The sensitive fiber is assembled in a syringe-like device. Like this, the matrix is exposed into the headspace of any sample. Volatile molecules adsorb selectively on the matrix. They can be released from the fiber by thermal desorption or via solvent extraction. The application of SPME technique for volatile analysis was prevalently described in the last two decades (Vas \& Vékey, 2004; Kataoka, 2000; Roberts et al., 2000; Zhang \& Pawliszyn, 1993).

The most common way of volatile analysis is by gas chromatography (GC), which is usually combined with mass spectrometry (GC-MS). The obtained total ion chromatograms (TIC) comprise information about the retention time and mass spectra of each compound (mass-tocharge, $\mathrm{m} / \mathrm{z}$ ). Commercial mass spectral databases (i.e. NIST or Wiley libraries) can be employed for the identification of compounds. In this case, it is strongly recommended to calculate the Kováts' retention index (KI) of a separated compound on the basis of a homologous series of $n$-alkanes as an additional parameter of identity. Other common detection systems are flame ionization detectors (FID), which are used for quantitative purposes, or time-of-flight (TOF)-MS detectors that are used for the identification of overlapping peaks, obtained from a fast GC procedure (Tholl et al., 2006).

Beside the precise but very time-consuming and expensive analysis by GC-MS, portable electronic sensors (E-noses) can also potentially detect specific VOCs. They are typically constructed as multisensor arrays consisting of different types of chemo-resistive metal oxide sensors (i.e. $\mathrm{In}_{2} \mathrm{O}_{3}, \mathrm{SnO}_{2}$ ) (Presicce et al., 2006; Abramson et al., 2005, Falasconi et al., 2005, Dickinson et al., 1998). Gobbi et al. (2011) and Falasconi et al. (2005) investigated the use of an electronic nose system for the prediction of fumonisin contamination of maize in vitro. Olsson et al. (2002) used E-noses and GC-MS for the detection of Ochratoxin A and DON in barley (Hordeum vulgare L.) grains. Balasubramanian et al. (2007) applied E-noses for the classification among barley samples, based on their ergosterol content.

The ion mobility spectrometry (IMS) has become a useful tool for mobile volatile analysis. The procedure is based on the velocity of gas-phase ions in an electric field under atmospheric pressure. So far, the technique, which is also available in portable devices, has been used for 
the detection of biohazards, chemical weapons, and also for environmental studies (Kolakowski \& Mester, 2007; $\quad$ Borsdorf \& Eiceman, 2006; $\quad$ Eiceman \& Karpas, 2005; Guevremont, 2004).

The emission of volatiles from infected maize and small-grain cereals by pathogenic fungi was extensively studied over the last decades. In this context, terpenoid compounds that are mainly released from plants become more important (Gershenzon \& Dudareva, 2007). Terpenoids, also known as isoprenoids, are the largest family of natural compounds (> 30000 molecules). They derive from the mevalonate pathway in the cytosol (Aharoni et al., 2005). So far, a large number of natural monterpenes $\left(C_{10}\right)$, sesquiterpenes $\left(C_{15}\right)$, diterpenes $\left(C_{20}\right)$ and triterpenes $\left(\mathrm{C}_{30}\right)$ are known. The large structural diversity of terpenes is caused by the high number of enzymes that catalyse their formation (terpene synthases) and the fact that they are able to generate multiple products from a single substrate (Degenhardt et al., 2009). Especially maize produces a multitude of terpenoid compounds (Schnee et al., 2006). Koellner et al. (2008) described the production of $\beta$-bisabolene and $\beta$-macrocarpene (first described by Cool (2005)) by the terpene synthases TPS6 and TPS11 in maize. Recently, Huffaker et al. (2011) perceived the formation of non-volatile terpenoid phytoalexins, so called zealexins, that resemble $\beta$-macrocarpene. The authors investigated maize tissue that was exposed to herbivory or fungal infection. Furthermore, a class of diterpenoid phytoalexins, so called kauralexins, was currently described in maize (Schmelz et al., 2011). The recent findings illustrate the relevance of terpenoid compounds in plant defense mechanisms.

In the specific case of Fusarium, the sesquiterpene hydrocarbon trichodiene has been identified as a volatile marker for the biosynthesis of fungal derived trichothecenes. This was first described by Jeleń et al. (1997), who cultivated different Fusarium spp. on autoclaved wheat grains. For a long time, the production of trichodiene was only reported in context with trichothecene producers. However, Dickschat et al. (2011) described trichodiene as a principal component in the volatile spectrum of $F$. verticillioides, a species that is not able to produce trichothecenes. The group analysed in vitro cultures on CM agar medium. Demyttenaere et al. (2004) reported the production of trichodiene and other sesquiterpenes by toxigenic Fusarium sp. on malt extract and potato dextrose agar. The group was able to distinguish between toxigenic strains on the basis of the terpene profile. Girotti et al. (2010) investigated the VOC emission of different Fusarium spp. growing on sterile rice cultures and 
could detect a broad spectrum of unknown sesquiterpenes beside trichodiene. They were also able to discriminate between species on the basis of the volatile profile.

It has to be considered that volatile profiles of in vitro cultures with fungi growing on sterile plant substrate differ considerably from profiles of infected living plants in the field or greenhouse. In vitro experiments with autoclaved plant substrate, consequently, do not render the plant response signals towards infection and fungal growth stays unchallenged. Apart from this aspect, the choice of medium and nutrients (i.e. grains or artificial medium) as well as incubation conditions have an effect on the presence and concentration of certain volatile compounds (De Lucca et al., 2012; Christen et al., 1997; Wheatley et al., 1997). Hence, under in vitro conditions the attack-defense interaction and associated VOC emission between fungus and plant cannot be described properly. For the first time Perkowski et al. (2008) monitored the volatile emission of wheat and triticale grains, infected with $F$. culmorum, under field conditions. They could detect trichodiene and no other relevant terpenoid compound under these conditions. Girotti et al. (2012) were able to predict an early F. graminearum infection of wheat and, respectively, the contamination with DON and its derivates from field samples via SPME/GC-MS. The authors were mainly referring to trichodiene. There is no in vivo investigation of the VOC emission on Fusarium infected maize ears, including the identification of specific volatile markers for infection to this day.

\subsubsection{Other Secondary Metabolites Involved in Plant Defense Reactions}

Injury by insects or pathogens can activate defense mechanisms in the plant. These protective processes include the induction of secondary metabolites, such as glucosinolates, phenolics, terpenoid compounds and phytoalexins (Bennett \& Wallsgrove, 1994).

Plant oxylipins play a role in the defense response to pathogen attack. They are oxidized lipophilic molecules that derive from the oxidation of polyunsaturated fatty acids (PUFAS), such as linoleic acid, $\alpha$-linolenic acid and roughanic acid (Goebel \& Feussner, 2009; Howe \& Schilmiller, 2002; Brash, 1999). The formation of plant oxylipins occurs by either non-enzymatic or enzymatic reactions. The non-enzymatic synthesis is the peroxidation of membrane lipids upon oxidative stress (Goebel et al., 2003). The enzymatic reaction is the incorporation of molecular oxygen into free fatty acids by lipoxygenases (LOX) (Feussner \& Wasternack, 2002). Plant oxylipins show a high structural diversity. They occur as free molecules in the cytosol, stroma and peroxisomal matrix. Furthermore, they are bound 
in complex lipids. Oxylipins in plants have signalling functions (i.e. jasmonic acid and its methyl ester) and antimicrobial, antifungal and anti-insecticidal activities (i.e. leaf aldehydes, divinyl ethers) (Prost et al., 2005; Reymond \& Farmer, 1998). The analysis of oxylipins is possible via fluorescence detection or by liquid and gas chromatography, as described in detail by Goebel \& Feussner (2009). The formation of oxylipins, as compounds involved in development and response, also plays a role in other organisms, such as mammals and fungi. The occurrence of oxylipins in fungi was recently reviewed by Brodhun \& Feussner (2011). Up to now, the regulation of oxylipins in infected and non-infected maize ears is unknown.

Phytoalexins are a group of inducible compounds with antifungal and antibacterial properties. They are synthesized in plant tissues close to the site of infection (Bennett \& Wallsgrove, 1994). As mentioned earlier, a novel class of phytoalexins, so called zealexins, was described by Huffaker et al. (2011). Zealexins are non-volatile acidic sesquiterpenoid hydrocarbons. Their skeleton resembles $\beta$-macrocarpene, a terpenoid volatile compound. Zealexins are known to play a role in the chemical defense of injured maize plants, i.e. during pathogen and herbivore attack. Furthermore, the authors describe an induction under exposition with stress hormones (i.e. jasmonic acid and ethylene). The concentration of zealexins reveals a positive correlation with the transcript levels of terpene synthase genes tps 6 and tps 11 . These terpene synthases catalyse the formation of $\beta$-bisabolene and $\beta$-macrocarpene, the volatile zealexin precursors, in several plant tissues (Koellner et al., 2008). The analysis of zealexins can be accomplished by GC-MS after derivatization.

\subsection{A Fungal Antagonist of Fusarium spp. and its Potential Use for the Specific Detection of Zearalenone}

\subsubsection{The Mycoparasite Gliocladium roseum, its Features and its Use as a Biocontrol Agent Against Phytopathogenic Fungi}

The parasitism of fungi, named mycoparasitism, occurs in a biotrophic mode, with little harm to the host fungus, or in a destructive mode. In the latter case, the parasite kills the fungal host with toxic secondary metabolites, i.e. toxins and antibiotics (Barnett \& Lilly, 1962). 
The common mycoparasite Gliocladium roseum Bainier (syn. Clonostachys rosea (Link: Fr.) Schoers, Samuels, Siefert and W. Gams; teleomorph Bionectria ochroleuca) occurs worldwide as a non-pathogenic parasite in vegetative and generative tissues of plants, but is also associated with nematodes and resting structures of phytopathogenic fungi in the soil (Rodríguez et al., 2011; Zhang et al., 2008). The hyphomycete produces one-celled conidia on two different types of conidiophores (Sutton et al., 1997). The activity of cell wall-degrading enzymes from G. roseum might play a key role in its mycoparasitic activity (Roberti et al., 2002). Besides glucanases (Chatterton \& Punja, 2009), the fungus releases chitinases that catalyze the decomposition of chitin, the major component of fungal cell walls and the second most abundant polysaccharide in the world after cellulose (Mamarabadi et al., 2008; Gan et al., 2007). Apart from G. roseum, a multitude of other organisms are able to produce chitinases (Inglis \& Kawchuk, 2002).

The cyclopentapeptide argifin, which is produced by Gliocladium sp. was investigated by Omura et al. (2000) and Shiomi et al. (2000). Argifin is a chitinase inhibitor and can suppress the maturation and reproduction of insects. It is also known as an inhibitor of chitinase B1 in Aspergillus fumigatus (Dixon et al., 2005). Its application against fungi and insects was discussed extensively (Hirose, 2012, Rush et al., 2010).

As mentioned earlier, the estrogenic mycotoxin ZEN is produced by phytopathogenic Fusarium spp., mainly $F$. graminearum and $F$. culmorum. It was assumed that it plays a role during competition, because the toxin has an inhibitory effect on the growth of other filamentous fungi than G. roseum (Utermark \& Karlovsky, 2007). The mycoparasite G. roseum protects itself from the inhibitory effects of ZEN by the secretion of a specific zearalenone lactonase (el-Sharkawy \& Abul-Hajj, 1988). This enzyme converts ZEN to a cleavage product (1-(3,5-dihydroxyphenyl)-10'-hydroxy-1'E-undecene-6'-one), which has no estrogenic activity. The gene that encodes the specific lactonase was described by two distinct research groups (Kakeya et al., 2002; Takahashi-Ando et al., 2002; E. H. Crane, J. T. Gilliam, P. Karlovsky, and J. R. Maddox, 3 October 2002, World Trade Organization patent application 02/ 076205; P. Karlovsky, E. H. Crane, J. T. Gilliam, and J. R. Maddox, U.S. patent application 20030073239). Recently, the ability of the yeast Trichosporon mycotoxinivorans to detoxify ZEN was described by Vekiru et al. (2010).

G. roseum was extensively studied as a biocontrol agent against phytopathogenic fungi. Its application against the important pathogen Botrytis cinerea Pers. ex. Fr., a producer of grey 
mould in fruits and vegetables, was described (Morandi et al., 2000; Sutton et al., 1997). Teperi et al. (1998) screened several fungal isolates for their ability to control seed-borne $F$. culmorum infection in wheat and found isolates of $G$. roseum to be the most effective antagonists. Luongo et al. (2005) showed the use of G. roseum cultures to colonize and decompose wheat and maize debris in agricultural soils and, therefore, to inhibit the development of Fusarium spp. on these plant residues. Apart from its application as biocontrol agent, it was reported that G. roseum has a growth promoting effect on its host plant and enhances productivity. This effect might be stimulated by its antifungal abilities or by a direct interaction between the mycoparasite and the plant (Sutton et al., 2006).

\subsubsection{Bioassays for the Identification of Estrogenic Zearalenone}

Because the analysis of ZEN and other estrogenic compounds by liquid chromatography is comparatively expensive, it was attempted to develop cheaper bioassays. A bioluminescence assay, based on Photobacterium phosphoreum for several mycotoxins, including ZEN, was presented by Yates \& Porter (1982). A bioassay, based on MCF-7 cells (breast cancer cell line), for estrogens in feedstuff was reported by Welshons et al. (1990). The group investigated common estrogens, like estradiol and ZEN, as well as phytoestrogens (i.e. genistein) and could not specifically distinguish between different estrogenic compounds. Mayr et al. (1992) presented a bioassay for myco- and phytoestrogens in cereal extracts on the basis of engineered susceptible cell lines that exhibited estrogen-specific proteins. An engineered yeast assay (strain: Saccharomyces cerevisiae, YZRM7), based on human estrogen receptors, was used by Mitterbauer et al. (2003) for the detection of estrogenic compounds from cereal extracts. Besides ZEN and its derivates, their assay also responded to $17-\beta$ estradiol. Bovee et al. (2004) constructed a yeast bioassay, based on the fusion of human estrogen receptors $\alpha$ and $\beta$ with green fluorescent protein (GFP). Their assay showed a broad response to estrogenic compounds, including ZEN. Later, the group tested their assay with contaminated animal feed and could successfully detect hormonal substances (Bovee et al. (2006). Winter et al. (2008) established a bioassay for estrogens on the basis of the human estrogen receptors $\alpha$ and $\beta$ in embryonal kidney cells. They detected phytoestrogens and other extrogenic compounds, including ZEN, in the diet of pigs.

As mentioned earlier, pigs are highly susceptible to ZEN and there is an urgent need to prevent the transfer of ZEN contaminated agricultural products to their diet (Marin et al., 2010; D’Mello et al., 1999). The protein demand in pig nutrition is, nowadays, 
mainly covered with soy, which contains huge amounts of phytoestrogens (Winter et al., 2008). Hence, to identify a true contamination with estrogenic Fusarium metabolites it is necessary not only to establish a bioassay for estrogenic compounds, but to provide a specific assay with respect to ZEN and its derivates. All previously described assays, based on the human estrogen receptors $\alpha$ and $\beta$, respond to physiological estrogens as well as to phyto- and mycoestrogens (Kuiper et al., 1998; Martin et al., 1978).

The use of the mycoparasite G. roseum for the detection of ZEN was reported by Utermark \& Karlovsky (2006). The group used an engineered G. roseum strain, carrying a fusion of the ZEN sensing promoter element zes2 and a reporter gene (GFP), to compare the use of a real-time PCR cycler and a common fluorescence reader. They detected an increase of the fluorescence signal with increasing ZEN concentration.

\subsection{Aims of the Study}

To prevent the transfer of contaminated material from the field to the food chain of animals and humans is a major goal of applied Fusarium research and there is an urgent need for a reliable method for the early and fast detection of Fusarium infections in pre- and postharvest processes. With the exception of the visual observation of cereal crops by farmers and experts, there is no efficient system for the early detection of Fusarium infection in fields until now. The exact determination of fungal biomass by molecular methods (i.e. quantitative real-time polymerase chain reaction, PCR) and the determination of toxin contamination with analytical methods (high performance liquid chromatography/mass spectrometry, HPLC-MS) is laborious, expensive and not adequate as fast decision guidance for farmers and traders (Magan et al., 2000).

Volatile organic compounds provide a potential alternative to detect Fusarium infection in crops. The purpose of this study was to investigate the suitability of a fast detection system for Fusarium infected wheat and maize on the basis of specific volatile biomarkers. Moreover, we wanted to optimize a simple and inexpensive bioassay for the detection and quantification of the estrogenic mycotoxin zearalenone in agricultural commodities. These methods can be used as risk-management tools in modern agriculture and food production. 


\section{References}

Abramson D, Hulasare R, York RK, et al. (2005) Mycotoxins, ergosterol, and odor volatiles in durum wheat during granary storage at $16 \%$ and $20 \%$ moisture content. Journal of Stored Products Research. 41:67-76.

Aharoni A, Jongsma M, Bouwmeester H (2005) Volatile science? Metabolic engineering of terpenoids in plants. Trends in Plant Science. 10:594-602.

Anonymus (2007) Commission regulation (EC) no. 1126/2007amending Regulation (EC) No $1881 / 2006$ setting maximum levels for certain contaminants in foodstuffs as regards Fusarium toxins in maize and maize products. Official Journal of the European Union 255:14-17.

Arimura G, Kost C, Boland W (2005) Herbivoreinduced, indirect plant defences. Biochimica et Biophysica Acta (BBA) Molecular and Cell Biology of Lipids. 1734:91-111.

Arthur CL, Pawliszyn J (1990) Solid phase microextraction with thermal desorption using fused silica optical fibers. Analytical Chemistry. 62:2145-2148.

Bacon CW, Hinton DM (1996) Symptomless endophytic colonization of maize by Fusarium moniliforme. Canadian Journal of Botany. 74:1195-1202.

Bakkali F, Averbeck S, Averbeck D, Idaomar M (2008) Biological effects of essential oils A review. Food and Chemical Toxicology. 46:446-475.

Balasubramanian S, Panigrahi S, Kottapalli B, Wolf-Hall CE (2007) Evaluation of an artificial olfactory system for grain quality discrimination. LWT - Food Science and Technology. 40:1815-1825.

Baldwin IT, Halitschke R, Paschold A (2006) Volatile Signaling in Plant-Plant Interactions: 'Talking Trees' in the Genomics Era. Science. 311:812-815.

Baratta MT, Dorman, HJD, Deans SG, et al. (1998) Antimicrobial and antioxidant properties of some commercial essential oils. Flavour and Fragrance Journal. 13:235-244.

Barnett HL, Lilly VG (1962) A destructive mycoparasite, Gliocladium roseum. Mycologia 54:72-77.
Bartelt RJ, Wicklow DT (1999) Volatiles from Fusarium verticillioides (Sacc.) Nirenb. and Their Attractiveness to Nitidulid Beetles. Journal of Agricultural and Food Chemistry. 47:2447-2454.

Bate NJ, Rothstein SJ (1998) C6-volatiles derived from the lipoxygenase pathway induce a subset of defense-related genes. The Plant Journal. 16:561-569.

Bennett JW, Klich M (2003) Mycotoxins. Clinical Microbiology Reviews. 16:497-516.

Bennett RN, Wallsgrove RM (1994) Tansley review no. 72. Secondary metabolites in plant defence mechanisms. New Phytologist. 127:617-633.

Bestmann HJ, Winkler L, Von Helversen O (1997) Headspace analysis of volatile flower scent constituents of bat-pollinated plants. Phytochemistry. 46:1169-1172.

Blandino M, Pilati A, Reyneri A, Scudellari D (2010) Effect of maize crop residue density on Fusarium head blight and on deoxynivalenol contamination of common wheat grains. Cereal Research Communications. 38:550-559.

Boerjesson T, Stoellman U, Adamek, P, Kaspersson A (1989) Analysis of volatile compounds for detection of molds in stored cereals. Cereal Chemistry. 66:300-304.

Borsdorf H, Eiceman GA (2006) Ion Mobility Spectrometry: Principles and Applications. Applied Spectroscopy Reviews. 41:323375.

Bottalico A, Perrone G (2002) Toxigenic Fusarium species and mycotoxins associated with head blight in small-grain cereals in Europe. European journal of plant pathology 108:611-624.

Bovee TFH, Bor G, Heskamp HH, et al. (2006) Validation and application of a robust yeast estrogen bioassay for the screening of estrogenic activity in animal feed. Food Additives and Contaminants. 23:556-568. 
Bovee TFH, Helsdingen RJR, Rietjens IMCM, et al. (2004) Rapid yeast estrogen bioassays stably expressing human estrogen receptors $\alpha$ and $\beta$, and green fluorescent protein: a comparison of different compounds with both receptor types. The Journal of Steroid Biochemistry and Molecular Biology. 91:99-109.

Brash AR (1999) Lipoxygenases: Occurrence, Functions, Catalysis, and Acquisition of Substrate. Journal of Biological Chemistry. 274:23679-23682.

Brodhun F, Feussner I (2011) Oxylipins in fungi. FEBS Journal. 278:1047-1063.

Buttery RG, Ling LC (1995) Volatile Flavor Components of Corn Tortillas and Related Products. Journal of Agricultural and Food Chemistry. 43:1878-1882.

Chatterton S, Punja ZK (2009) Chitinase and $\beta$-1,3glucanase enzyme production by the mycoparasite Clonostachys rosea f. catenulata against fungal plant pathogens. Canadian Journal of Microbiology. 55:356-367.

Christen P, Meza JC, Revah S (1997) Fruity aroma production in solid state fermentation by Ceratocystis fimbriata: influence of the substrate type and the presence of precursors. Mycological Research. 101:911-919.

Cool LG (2005) Sesquiterpenes from Cupressus macrocarpa foliage. Phytochemistry. 66:249-260.

D’Mello JPF, Placinta CM, Macdonald AMC (1999) Fusarium mycotoxins: a review of global implications for animal health, welfare and productivity. Animal Feed Science and Technology. 80:183-205.

Daferera DJ, Ziogas BN, Polissiou MG (2000) GCMS Analysis of Essential Oils from Some Greek Aromatic Plants and Their Fungitoxicity on Penicillium digitatum. Journal of Agricultural and Food Chemistry. 48:2576-2581.

Degenhardt J, Koellner TG, Gershenzon J (2009) Monoterpene and sesquiterpene synthases and the origin of terpene skeletal diversity in plants. Phytochemistry 70:1621-1637.

Demyttenaere JC, Moriña RM, De Kimpe N, Sandra P (2004) Use of headspace solidphase microextraction and headspace sorptive extraction for the detection of the volatile metabolites produced by toxigenic Fusarium species. Journal of Chromatography A. 1027:147-154.
Desjardins AE (2003) Gibberella From a(venaceae) To $z($ eae $)$ Annual Review of Phytopathology. 41:177-198.

Desjardins AE, Plattner RD (2000) Fumonisin B1 Nonproducing Strains of Fusarium verticillioides Cause Maize (Zea mays) Ear Infection and Ear Rot. Journal of Agricultural and Food Chemistry. 48:5773-5780.

Dicke M, Agrawal AA, Bruin J (2003) Plants talk, but are they deaf? Trends in Plant Science. $8: 403-405$.

Dicke M, Vanbeek TA, Posthumus MA, et al. (1990) Isolation and identification of volatile kairomone that affects acarine predatorprey interactions Involvement of host plant in its production. Journal of Chemical Ecology. 16:381-396.

Dickinson TA, White J, Kauer JS, Walt DR (1998) Current trends in 'artificial-nose' technology. Trends in Biotechnology. 16:250-258.

Dickschat JS, Brock NL, Citron CA, Tudzynski B (2011) Biosynthesis of Sesquiterpenes by the Fungus Fusarium verticillioides. ChemBioChem. 12:2088-2095.

Dickschat JS, Wenzel SC, Bode HB, et al. (2004) Biosynthesis of Volatiles by the Myxobacterium Myxococcus xanthus. ChemBioChem. 5:778-787.

Dill-Macky R, Jones RK (2000) The Effect of Previous Crop Residues and Tillage on Fusarium Head Blight of Wheat. Plant Disease. 84:71-76.

Dixon MJ, Andersen OA, Van Aalten, DMF, Eggleston IM (2005) An efficient synthesis of argifin: A natural product chitinase inhibitor with chemotherapeutic potential. Bioorganic \& Medicinal Chemistry Letters. 15:4717-4721.

Donath J, Boland W (1995) Biosynthesis of acyclic homoterpenes: enzyme selectivity and absolute configuration of the nerolidol precursor. Phytochemistry 39:785-790.

Doohan FM, Brennan J, Cooke BM (2003) Influence of climatic factors on Fusarium species pathogenic to cereals. European Journal of Plant Pathology. 109:755-768.

Edwards SG (2004) Influence of agricultural practices on Fusarium infection of cereals and subsequent contamination of grain by trichothecene mycotoxins. Toxicology Letters. 153:29-35. 
Eiceman GA, Karpas Z (2005) Ion mobility spectrometry. CRC Press, Boca Raton.

el-Sharkawy S, Abul-Hajj YJ (1988) Microbial cleavage of zearalenone. Xenobiotica 18:365-371.

Entwistle G, Bachelor S, Booth E, Walker K (1998) Economics of starch production in the UK. Industrial Crops and Products. 7:175-186.

Falasconi M, Gobbi E, Pardo M, et al. (2005) Detection of toxigenic strains of Fusarium verticillioides in corn by electronic olfactory system. Sensors and Actuators B: Chemical. 108:250-257.

Faridi H, Finley JW, D'Appolonia B (1989) Improved wheat for baking. Critical Reviews in Food Science and Nutrition. 28:175-209.

Feussner I, Wasternack C (2002) The Lipoxygenase Pathway. Annual Review of Plant Biology. 53:275-297.

Food and Agriculture Organization of the United Nations - FAOSTAT. 2012. Available at: http://faostat.fao.org/?lang=en [Accessed August 23, 2012]

Forsell JH, Jensen R, Tai J-H, et al. (1987) Comparison of acute toxicities of deoxynivalenol (vomitoxin) and 15acetyldeoxynivalenol in the B6C3F1 mouse. Food and Chemical Toxicology. 25:155-162.

Gan Z, Yang J, Tao N, et al. (2007) Cloning and expression analysis of a chitinase gene Crchil from the mycoparasitic fungus Clonostachys rosea (syn. Gliocladium roseum). Journal of Microbiology-Seoul 45:422.

Gelderblom WC, Jaskiewicz K, Marasas, WF, et al. (1988) Fumonisins-novel mycotoxins with cancer-promoting activity produced by Fusarium moniliforme. Applied and Environmental Microbiology. 54:18061811.

Gerlach W, Nirenberg H (1982) The genus Fusarium - a pictorial atlas. BerlinDahlem: Mitteilungen aus der Biologischen Bundesanstalt fuer Landund Forstwirtschaft.

Gershenzon J, Dudareva N (2007) The function of terpene natural products in the natural world. Nature Chemical Biology. 3:408414.
Gilbert J, Fernando WGD (2004) Epidemiology and biological control of Gibberella zeae / Fusarium graminearum. Canadian Journal of Plant Pathology. 26:464-472.

Girotti JR, Malbrán I, Lori GA, Juárez MP (2010) Use of solid phase microextraction coupled to capillary gas chromatographymass spectrometry for screening Fusarium spp. based on their volatile sesquiterpenes. World Mycotoxin Journal. 3:121-128.

Girotti JR, Malbrán I, Lori GA, Juárez MP (2012) Early detection of toxigenic Fusarium graminearum in wheat. World Mycotoxin Journal. 5:143-152.

Gobbi E, Falasconi M, Torelli E, Sberveglieri G (2011) Electronic nose predicts high and low fumonisin contamination in maize cultures. Food Research International. 44:992-999.

Goebel C, Feussner I (2009) Methods for the analysis of oxylipins in plants. Phytochemistry. 70:1485-1503.

Goebel C, Feussner I, Rosahl S (2003) Lipid Peroxidation during the Hypersensitive Response in Potato in the Absence of 9Lipoxygenases. Journal of Biological Chemistry. 278:52834-52840.

Goswami RS, Kistler HC (2004) Heading for disaster: Fusarium graminearum on cereal crops. Molecular Plant Pathology. 5:515525.

Gouinguene SP, Turlings TCJ (2002) The Effects of Abiotic Factors on Induced Volatile Emissions in Corn Plants. Plant Physiology. 129:1296-1307.

Grob K (1973) Organic substances in potable water and in its precursor. Journal of Chromatography A. 84:255-273.

Guevremont R (2004) High-field asymmetric waveform ion mobility spectrometry: A new tool for mass spectrometry. Journal of Chromatography A. 1058:3-19.

Hirose T (2012) Study on the discovery of novel chitinase inhibitors based on natural products. Yakugaku Zasshi 132:10011010.

Holopainen JK, Gershenzon J (2010) Multiple stress factors and the emission of plant VOCs. Trends in plant science 15:176184.

Howe GA, Schilmiller AL (2002) Oxylipin metabolism in response to stress. Current Opinion in Plant Biology. 5:230-236. 
Huffaker A, Kaplan F, Vaughan MM, et al. (2011) Novel acidic sesquiterpenoids constitute a dominant class of pathogen-induced phytoalexins in maize. Plant physiology 156:2082-2097.

Inglis GD, Kawchuk LM (2002) Comparative degradation of oomycete, ascomycete, and basidiomycete cell walls by mycoparasitic and biocontrol fungi. Canadian Journal of Microbiology. 48:60-70.

Jeleń HH, Latus-Ziętkiewicz D, Wąsowicz E, Kamiński E (1997) Trichodiene as a volatile marker for trichothecenes biosynthesis. Journal of microbiological methods 31:45-49.

Kabeere F, Hampton J, Hill M (1997) Transmission of Fusarium graminearum (Schwabe) from maize seeds to seedlings. Seed Science and Technology. 25:245-252.

Kakeya H, Takahashi-Ando N, Kimura M, et al. (2002) Biotransformation of the mycotoxin, zearalenone, to a nonestrogenic compound by a fungal strain of Clonostachys sp. Bioscience, Biotechnology and Biochemistry 66:27232726.

Kaminski E, Stawicki S, Wasowicz E (1974) Volatile flavor compounds produced by molds of Aspergillus, Penicillium, and Fungi imperfecti. Applied microbiology. 27:1001-1004.

Kataoka H, Lord HL, Pawliszyn J (2000) Applications of solid-phase microextraction in food analysis. Journal of Chromatography A. 880:35-62.

Kesselmeier J, Staudt M (1999) Biogenic volatile organic compounds (VOC): an overview on emission, physiology and ecology. Journal of atmospheric chemistry. 33:2388.

Kessler A, Baldwin IT (2001) Defensive Function of Herbivore-Induced Plant Volatile Emissions in Nature. Science. 291:21412144.

Kimura M, Tokai T, Takahashi-Ando N, et al. (2007) Molecular and Genetic Studies of Fusarium Trichothecene Biosynthesis: Pathways, Genes, and Evolution. Bioscience, Biotechnology, and Biochemistry. 71:2105-2123.
Koellner TG, Schnee C, Li S, et al. (2008) Protonation of a Neutral (S)- $\beta$-Bisabolene Intermediate Is Involved in (S)- $\beta$ Macrocarpene Formation by the Maize Sesquiterpene Synthases TPS6 and TPS11. Journal of Biological Chemistry. 283:20779-20788.

Kolakowski BM, Mester Z (2007) Review of applications of high-field asymmetric waveform ion mobility spectrometry (FAIMS) and differential mobility spectrometry (DMS). The Analyst. 132:842-864.

Kramer R, Abraham W-R (2011) Volatile sesquiterpenes from fungi: what are they good for? Phytochemistry Reviews. 11:15-37.

Kuiper GGJM, Lemmen JG, Carlsson B, et al. (1998) Interaction of Estrogenic Chemicals and Phytoestrogens with Estrogen Receptor. Endocrinology. 139:4252-4263.

Kuiper-Goodman T, Scott PM, Watanabe H (1987) Risk assessment of the mycotoxin zearalenone. Regulatory Toxicology and Pharmacology. 7:253-306

Langevin F, Eudes F, Comeau A (2004) Effect of Trichothecenes Produced by Fusarium graminearum during Fusarium Head Blight Development in Six Cereal Species. European Journal of Plant Pathology 110:735-746.

Leslie JF, Bowden RL (2008) Fusarium graminearum: When species concepts collide. Cereal Research Communications 36:609-615.

Link HF (1809) Observationes in ordines plantarum naturals, Dissetatio I. Magazin fuer die neuesten Entdeckungen in der gesammten Naturkunde, Gesellschaft Naturforschender Freunde zu Berlin 3:342.

Linton CJ, Wright SJL (1993) Volatile organic compounds: microbiological aspects and some technological implications. Journal of Applied Microbiology 75:1-12.

Logrieco A, Mulé G, Moretti A, Bottalico A (2002) Toxigenic Fusarium species and mycotoxins associated with maize ear rot in Europe. European Journal of Plant Pathology 108:597-609.

Loreto F, Schnitzler JP (2010) Abiotic stresses and induced BVOCs. Trends in plant science 15:154-166. 
De Lucca AJ, Boue SM, Carter-Wientjes C, Bhatnagar D (2012) Volatile profiles and aflatoxin production by toxigenic and nontoxigenic isolates of Aspergillus flavus grown on sterile and non-sterile cracked corn. Annals of Agricultural and Environmental Medicine 19:91-98.

Luongo L, Galli M, Corazza L, et al. (2005) Potential of fungal antagonists for biocontrol of Fusarium spp. in wheat and maize through competition in crop debris. Biocontrol Science and Technology 15:229-242.

Magan N, Evans P (2000) Volatiles as an indicator of fungal activity and differentiation between species, and the potential use of electronic nose technology for early detection of grain spoilage. Journal of Stored Products Research 36:319-340.

Maiorano A, Blandino M, Reyneri A, Vanara F (2008) Effects of maize residues on the Fusarium spp. infection and deoxynivalenol (DON) contamination of wheat grain. Crop Protection 27:182-188.

Mamarabadi M, Jensen B, Luebeck M (2008) Three endochitinase-encoding genes identified in the biocontrol fungus Clonostachys rosea are differentially expressed. Current Genetics 54:57-70.

Marasas WF, Kellerman TS, Pienaar JG, Naudé TW (1976) Leukoencephalomalacia: a mycotoxicosis of Equidae caused by Fusarium moniliforme Sheldon. Onderstepoort Journal of Veterinary Research 43:113-122.

Marin DE, Taranu I, Burlacu R, Tudor DS (2010) Effects of zearalenone and its derivatives on the innate immune response of swine. Toxicon 56:956-963.

Marin S, Sanchis V, Magan N (1995) Water activity, temperature, and $\mathrm{pH}$ effects on growth of Fusarium moniliforme and Fusarium proliferatum isolates from maize. Canadian Journal of Microbiology 41:1063-1070.

Martin PM, Horwitz KB, Ryan DS, McGuire WL (1978) Phytoestrogen Interaction with Estrogen Receptors in Human Breast Cancer Cells. Endocrinology 103:18601867.

Mayr E (1963) Animal species and evolution. Harvard University Press, Cambridge
Mayr U, Butsch A, Schneider S (1992) Validation of two in vitro test systems for estrogenic activities with zearalenone, phytoestrogens and cereal extracts. Toxicology 74:135149.

McMullen M, Jones R, Gallenberg D (1997) Scab of Wheat and Barley: A Re-emerging Disease of Devastating Impact. Plant Disease 81:1340-1348.

Mérey G, Veyrat N, Mahuku G, et al. (2011) Dispensing synthetic green leaf volatiles in maize fields increases the release of sesquiterpenes by the plants, but has little effect on the attraction of pest and beneficial insects. Phytochemistry 72:1838-1847.

Miller SS, Reid LM, Harris LJ (2007) Colonization of maize silks by Fusarium graminearum, the causative organism of gibberella ear rot. Canadian Journal of Botany 85:369376.

Mitterbauer R, Weindorfer $\mathrm{H}$, Safaie $\mathrm{N}$, et al. (2003) A Sensitive and Inexpensive Yeast Bioassay for the Mycotoxin Zearalenone and Other Compounds with Estrogenic Activity. Applied and Environmental Microbiology 69:805-811.

Morandi MAB, Sutton JC, Maffia LA (2000) Effects of host and microbial factors on development of Clonostachys rosea and control of Botrytis cinerea in rose. European Journal of Plant Pathology 106:439-448.

Munkvold GP (2003) Epidemiology of Fusarium diseases and their mycotoxins in maize ears. European Journal of Plant Pathology 109:705-713.

Munkvold GP, McGee DC, Carlton WM (1997) Importance of different pathways for maize kernel infection by Fusarium moniliforme. Phytopathology 87:209-217.

Murillo-Williams A, Munkvold GP (2008) Systemic Infection by Fusarium verticillioides in Maize Plants Grown Under Three Temperature Regimes. Plant Disease 92:1695-1700.

Nirenberg HI, O’Donnell K (1998) New Fusarium Species and Combinations within the Gibberella fujikuroi Species Complex. Mycologia 90:434-458.

Nuss ET, Tanumihardjo SA (2010) Maize: A Paramount Staple Crop in the Context of Global Nutrition. Comprehensive Reviews in Food Science and Food Safety 9:417436. 
Nyvall RF (1970) Chlamydospores of Fusarium roseum "Graminearum" as Survival Structures. Phytopathology 60:1175-1177.

Olsson J, Boerjesson T, Lundstedt T, Schnuerer J (2002) Detection and quantification of ochratoxin A and deoxynivalenol in barley grains by GC-MS and electronic nose. International Journal of Food Microbiology 72:203-214.

Omura S, Arai N, Yamaguchi Y, et al. (2000) Argifin, a new chitinase inhibitor, produced by Gliocladium sp. FTD-0668. I. Taxonomy, fermentation, and biological activities. Journal of Antibiotics 53:603608.

Osborne LE, Stein JM (2007) Epidemiology of Fusarium head blight on small-grain cereals. International Journal of Food Microbiology 119:103-108.

Oslaj M, Mursec B, Vindis P (2010) Biogas production from maize hybrids. Biomass and Bioenergy 34:1538-1545.

Parry DW, Jenkinson P, McLEOD L (1995) Fusarium ear blight (scab) in small grain cereals - a review. Plant Pathology 44:207-238.

Parsons MW, Munkvold GP (2010) Associations of planting date, drought stress, and insects with Fusarium ear rot and fumonisin B1contamination in California maize. Food Additives \& Contaminants: Part A 27:591-607.

Paul PA, El-Allaf SM, Lipps PE, Madden LV (2004) Rain Splash Dispersal of Gibberella zeae Within Wheat Canopies in Ohio. Phytopathology 94:1342-1349.

Perkowski J, Busko M, Chmielewski J, et al. (2008) Content of trichodiene and analysis of fungal volatiles (electronic nose) in wheat and triticale grain naturally infected and inoculated with Fusarium culmorum. International Journal of Food Microbiology 126:127-134.

Placinta C., D’Mello JP., Macdonald AM. (1999) A review of worldwide contamination of cereal grains and animal feed with Fusarium mycotoxins. Animal Feed Science and Technology 78:21-37.

Presicce DS, Forleo A, Taurino AM, et al. (2006) Response evaluation of an E-nose towards contaminated wheat by Fusarium poae fungi. Sensors and Actuators B: Chemical 118:433-438.
Proctor RH (1995) Reduced Virulence of Gibberella zeae Caused by Disruption of a Trichothecene Toxin Biosynthetic Gene. Molecular Plant-Microbe Interactions 8:593-601.

Proctor RH, Desjardins AE, McCormick SP, et al. (2002) Genetic analysis of the role of trichothecene and fumonisin mycotoxins in the virulence of Fusarium. European Journal of Plant Pathology 108:691-698.

Prost I, Dhont S, Rothe G, et al. (2005) Evaluation of the Antimicrobial Activities of Plant Oxylipins Supports Their Involvement in Defense against Pathogens. Plant Physiology 139:1902-1913.

Reid LM, Nicol RW, Ouellet T, et al. (1999) Interaction of Fusarium graminearum and F. moniliforme in Maize Ears: Disease Progress, Fungal Biomass, and Mycotoxin Accumulation. Phytopathology 89:10281037.

Reymond P, Farmer EE (1998) Jasmonate and salicylate as global signals for defense gene expression. Current Opinion in Plant Biology 1:404-411.

Roberti R, Zakrisson E, Flamigni F, et al. (2002) Antagonistic fungi producing hydrolytic enzymes, active in degrading the cell wall of some foot rot pathogens (Fusarium spp.) of wheat. Journal of Plant Diseases and Protection 109:101-108.

Roberts DD, Pollien P, Milo C (2000) Solid-Phase Microextraction Method Development for Headspace Analysis of Volatile Flavor Compounds. Journal of Agricultural and Food Chemistry 48:2430-2437.

Rodríguez MA, Cabrera G, Gozzo FC, et al. (2011) Clonostachys rosea BAFC3874 as a Sclerotinia sclerotiorum antagonist: mechanisms involved and potential as a biocontrol agent. Journal of Applied Microbiology 110:1177-1186.

Rotter BA (1996) Invited review: Toxicology of deoxynivalenol (vomitoxin). Journal of Toxicology and Environmental Health 48:1-34.

Rush CL, Schuettelkopf AW, Hurtado-Guerrero R, et al. (2010) Natural product-guided discovery of a fungal chitinase inhibitor. Chemistry and Biology 17:1275-1281.

Samson RA, Gams W (1984) The taxonomic situation in the hyphomycete genera Penicillium, Aspergillus and Fusarium. Antonie van Leeuwenhoek 50:815-824. 
Schmelz EA, Kaplan F, Huffaker A, et al. (2011) Identity, regulation, and activity of inducible diterpenoid phytoalexins in maize. Proceedings of the National Academy of Sciences 108:5455-5460.

Schnee C, Koellner TG, Held M, et al. (2006) The products of a single maize sesquiterpene synthase form a volatile defense signal that attracts natural enemies of maize herbivores. Proceedings of the National Academy of Sciences of the United States of America 103:1129-1134.

Schulthess F, Cardwell KF, Gounou S (2002) The Effect of Endophytic Fusarium verticillioides on Infestation of Two Maize Varieties by Lepidopterous Stemborers and Coleopteran Grain Feeders. Phytopathology 92:120-128.

Shiferaw B, Prasanna BM, Hellin J, Baenziger M (2011) Crops that feed the world 6. Past successes and future challenges to the role played by maize in global food security. Food Security 3:307-327.

Shiomi K, Arai N, Iwai Y, et al. (2000) Structure of argifin, a new chitinase inhibitor produced by Gliocladium sp. Tetrahedron Letters 41:2141-2143.

Soliman K., Badeaa R. (2002) Effect of oil extracted from some medicinal plants on different mycotoxigenic fungi. Food and Chemical Toxicology 40:1669-1675.

Summerell BA, Laurence MH, Liew ECY, Leslie JF (2010) Biogeography and phylogeography of Fusarium: a review. Fungal Diversity 44:3-13.

Sutton JC, Liu W, Ma J, et al. (2006) Evaluation of the fungal endophyte Clonostachys rosea as an inoculant to enhance growth, fitness and productivity of crop plants. IV International Symposium on Seed, Transplant and Stand Establishment of Horticultural Crops; Translating Seed and Seedling 782. pp 279-286

Sutton JC, Li DW, Peng G, et al. (1997) Gliocladium roseum a versatile adversary of Botrytis cinerea in crops. Plant Disease 81:316-328.

Takahashi-Ando N, Kimura M, Kakeya H, et al. (2002) A novel lactonohydrolase responsible for the detoxification of zearalenone: enzyme purification and gene cloning. Biochemistry J 365:1-6.
Taylor JW, Jacobson DJ, Kroken S, et al. (2000) Phylogenetic Species Recognition and Species Concepts in Fungi. Fungal Genetics and Biology 31:21-32.

Teperi E, Keskinen M, Ketoja E, Tahvonen R (1998) Screening for fungal antagonists of seed-borne Fusarium culmorum on wheat using in vivo tests. European journal of plant pathology 104:243-251.

Thevis M, Fußhoeller G, Schaenzer W (2011) Zeranol: doping offence or mycotoxin? A case-related study. Drug Testing and Analysis 3:777-783.

Tholl D, Boland W, Hansel A, et al. (2006) Practical approaches to plant volatile analysis. The Plant Journal 45:540-560.

Turlings TCJ, Tumlinson JH, Lewis WJ (1990) Exploitation of Herbivore-Induced Plant Odors by Host-Seeking Parasitic Wasps. Science 250:1251-1253.

Ueno Y (1984) Toxicological features of T-2 toxin and related trichothecenes. Fundamental and Applied Toxicology 4:124-132.

Utermark J, Karlovsky P (2007) Role of Zearalenone Lactonase in Protection of Gliocladium roseum from Fungitoxic Effects of the Mycotoxin Zearalenone. Applied and Environmental Microbiology 73:637-642.

Utermark J, Karlovsky P (2006) Quantification of green fluorescent protein fluorescence using real-time PCR thermal cycler. BioTechniques 41:150-154.

Vas G, Vékey K (2004) Solid-phase microextraction: a powerful sample preparation tool prior to mass spectrometric analysis. Journal of Mass Spectrometry 39:233-254.

Vekiru E, Hametner C, Mitterbauer R, et al. (2010) Cleavage of Zearalenone by Trichosporon mycotoxinivorans to a Novel Nonestrogenic Metabolite. Applied and Environmental Microbiology 76:23532359.

Vichi S, Guadayol J, Caixach J, et al. (2007) Comparative study of different extraction techniques for the analysis of virgin olive oil aroma. Food Chemistry 105:11711178.

Welshons WV, Rottinghaus GE, Nonneman DJ, et al. (1990) A sensitive bioassay for detection of dietary estrogens in animal feeds. Journal of veterinary diagnostic investigation 2:268-273. 
Wheatley R, Hackett C, Bruce A, Kundzewicz A (1997) Effect of substrate composition on production of volatile organic compounds from Trichoderma spp. Inhibitory to wood decay fungi. International Biodeterioration \& Biodegradation 39:199-205.

Windels CE (2000) Economic and Social Impacts of Fusarium Head Blight: Changing Farms and Rural Communities in the Northern Great Plains. Phytopathology 90:17-21.

Winter P, Nau H, Lampen A, Kamphues J (2008) Detection of estrogenically active substances in diets for sows by an in vitro bioassay supported by HPLC analysis. Journal of Animal Physiology and Animal Nutrition 92:337-344.

Wollenweber HW, Reinking OA (1935) Die Fusarien, ihre Beschreibung, Schadwirkung und Bekaempfung. Paul Parey, Berlin
Yates IE, Porter JK (1982) Bacterial bioluminescence as a bioassay for mycotoxins. Applied and environmental microbiology 44:1072-1075.

Zhang L, Yang J, Niu Q, et al. (2008) Investigation on the infection mechanism of the fungus Clonostachys rosea against nematodes using the green fluorescent protein. Applied Microbiology and Biotechnology 78:983-990.

Zhang Z, Pawliszyn J (1993) Headspace solidphase microextraction. Analytical Chemistry 65:1843-1852.

Zinedine A, Soriano JM, Moltó JC, Mañes J (2007) Review on the toxicity, occurrence, metabolism, detoxification, regulations and intake of zearalenone: An oestrogenic mycotoxin. Food and Chemical Toxicology 45:1-18. 


\title{
2 Volatile Markers for the Infection of Maize (Zea mays L.) Ears with Fusarium spp.
}

\begin{abstract}
Volatile organic compounds (VOCs) belong to various chemical classes. They are known to mediate numerous interactions and play a role in plant response to abiotic and biotic stress, such as high temperature, drought and attack by pathogenic fungi and insects.

In the present study, we investigated the volatile profile of healthy and fungal-infected maize (Zea mays L.) ears under greenhouse conditions. For this purpose, maize ears were infected at the main flowering stage with several strains of Fusarium graminearum, F. verticillioides and $F$. subglutinans as well as mixtures of $F$. graminearum and $F$. verticillioides by injection of spore suspensions into the silk channel. Volatile profiles of maize ears were investigated at 24 dpi and in time series between 4 and 24 dpi. Sample collections were carried out by solidphase microextraction (SPME) and with a non-destructive open-loop-stripping (OLS) procedure. Analysis was carried out by gas chromatography mass spectrometry.

The volatile profiles of healthy and infected maize ears differed significantly and it was possible to identify a set of 27 volatile biomarkers for Fusarium infection. The marker set included common $\mathrm{C}_{5}$ to $\mathrm{C}_{8}$ volatiles and specific sesquiterpenoid compounds, such as $\alpha$ - and $\beta$-selinene, $\beta$-macrocarpene and $\beta$-bisabolene. Furthermore, trichodiene, the well known volatile marker for trichothecene production was found. Markers were detected within 4-8 dpi and their emission continued until early milk ripeness ( $24 \mathrm{dpi}$ ). The volatile biomarkers from infected maize ears were not found under in vitro conditions. To link volatile signatures with other defense and interaction mechanisms, we further assessed the formation of plant and fungal secondary metabolites, such as mycotoxins, zealexins and oxylipins. The results indicate a broad defense response of the plant towards pathogen attack.

Volatile biomarkers for Fusarium infection and associated mycotoxin contamination appear particularly useful for the early detection of fungal infection before disease symptoms become visible.
\end{abstract}




\subsection{Introduction}

Phytopathogenic fungi cause severe problems in agricultural crops each year. Fungal infections lead to considerable economic losses and to decreasing product quality by the contamination of food commodities with toxic metabolites (Bennett \& Klich, 2003; D’Mello et al., 1999). In this context Fusarium spp. are of particular interest. They play an important role in maize (Zea mays L.), but also in wheat (Triticum aestivum L.) and other small-grain cereals (Logrieco et al., 2002; Placinta et al., 1999). Maize ear rot is induced by a broad Fusarium species complex, including F. verticillioides (Sacc.) Nirenberg, F. graminearum Schwabe, F. avenaceum (Fr.) Sacc., F. subglutinans (Wollenweber \& Reinking) P.E. Nelson, Toussoun \& Marasas and F. proliferatum (Matsushima) Nirenberg (Dorn et al., 2009, Logrieco et al., 2002). Fusarium spp. produce secondary metabolites (mycotoxins) that exhibit multiple toxic effects in mammals and humans (Zinedine et al., 2007; Rotter, 1996; Gelderblom et al., 1991). Main Fusarium mycotoxins regularly found in maize, include trichothecenes, fumonisins, zearalenone and moniliformin (Glenn, 2007; Placinta et al., 1999).

Volatile organic compounds (VOCs) are hydrocarbons with a high vapour pressure. They are released by most organisms and belong to various chemical classes, such as alcohols, esters and terpenes. Due to their volatility, these molecules can travel large distances in heterogeneous environments (Tholl et al., 2006; Kesselmeier \& Staudt, 1999). Moreover, volatiles are known to mediate various ecological interactions (Dicke \& Baldwin, 2010; Wenke et al., 2009). The headspace of a plant-pathogen-system comprises volatiles that are likely to be emitted by the pathogen, such as 1-octen-3-ol and 3-octanone (Boerjesson et al., 1989), but also volatile signals that might be released by the host plant (i.e. 3-(Z)-hexenal) (Bate \& Rothstein, 1998). It has to be considered that a plant releases protective volatile signals not only upon pathogen or insect attack, but also upon abiotic stress, such as drought or high temperatures (Holopainen \& Gershenzon, 2010). The emission of volatiles from biotic or abiotic stress, can co-occur with the formation of other secondary metabolites involved in plant defense, such as zealexins, a class of non-volatile sesquiterpenoid phytoalexins (Huffaker et al., 2011), and plant oxylipins that derive from the oxidation of polyunsaturated fatty acids (PUFAS) and contribute to various plant defense mechanisms (Goebel \& Feussner, 2009). 
The use of volatiles for the recognition of fungal spoilage was extensively discussed in the last years (Turner \& Magan, 2004; Schnuerer et al., 1999; Jeleń \& Wąsowicz, 1998). So far, two common methods have been described for the collection of volatiles under controlled conditions, a dynamic and a static procedure. The time-consuming dynamic procedure (also loop-stripping), as described by Grob (1973) and Grob \& Zuercher (1976), is based on an air stream that circulates through a sample covering container and volatile molecules enrich on an adsorbing matrix (volatile trap). The dynamic procedure, which is considered as nondestructive, may be operated in a closed-loop or an open-loop mode (Tholl et al., 2006). The second procedure, the static volatile sampling, is based on an adsorbing matrix (i.e. polymer) that is exposed into a sealed container, which includes the sample. The solid-phase microextraction (SPME), which was first described by Arthur \& Pawliszyn (1990), is up to now the most common technique for the static sampling of volatiles (Jelen et al., 2012; Vas \& Vékey, 2004). After a volatile collection with either static or dynamic procedure, the trapped molecules can be released from the adsorbent material by solvent extraction or thermal desorption. Usually, the following analysis is carried out by gas chromatography mass spectrometry (GC-MS) (Tholl et al., 2006). Beside these sensitive bench-top techniques for volatile profiling and analysis, there are new transportable devices available, such as compact ion mobility spectrometers (IMS) (Borsdorf \& Eiceman, 2006) or physicochemical sensors, so called electronic-noses (Turner \& Magan, 2004). These devices enable the analysis of volatiles in the field and in post-harvest and storage processes (Campagnoli et al., 2011; Vautz et al., 2006).

Recent studies on the volatile emission of maize tissue, infected with Fusarium spp., report a broad volatile profile, including leaf alcohols and terpenoid compounds (Piesik et al., 2011). Of particular interest is the sesquiterpene trichodiene. Its use as a volatile marker for the biosynthesis of trichothecenes was first reported by Jeleń et al. (1997).

Until now, most approaches in context with Fusarium infection of crops are based on in vitro studies with fungi growing on sterile grain or artificial media (Dickschat et al., 2011; Girotti et al., 2010; Demyttenaere et al., 2004; Bartelt \& Wicklow, 1999). Under such conditions, the response of the host plant towards fungal infection (i.e. release of volatiles in terms of plant defense and plant-plant signaling) is excluded and, therefore, it is expected to find a more diverse volatile spectrum on live plants being infected with Fusarium species. Of particular interest is a specific set of volatile biomarkers to discriminate not only between 
'infected' and 'non-infected' fields/plants, but also between different Fusarium spp. and the associated spectrum of mycotoxins. Because an application of fungicides against Fusarium is only reasonable at early stages of infection, it has to be assessed how early these infection specific volatiles are detectable. These markers could be a useful tool for crop protection and consequently to limit the transfer of contaminated material to the food chain of humans and animals.

The objective of the present work is to investigate the volatile profiles of Fusarium infected maize ears over the time and with different sampling strategies to establish a reliable set of volatile markers for the infection of maize with toxigenic Fusarium species. Furthermore, the induction of secondary metabolites from the plant and the fungus (i.e. mycotoxins, compounds of plant defense) has to be examined and linked with the emission of volatile compounds. 


\subsection{Materials and Methods}

\subsubsection{Plant Material and Growth Conditions}

Experiments were performed on two maize varieties, including commercial hybrid maize (Ronaldinio, obtained from KWS Saat AG, Einbeck, Germany) and a dwarf maize cultivar (Gaspe Flint landrace, originally collected in Quebec, Canada, provided by Prof. Dr. J. Schirawski, Department for Molecular Biology of Plant-Microbe Interaction, University of Goettingen, Germany).

Hybrid maize was grown under greenhouse conditions at $26 \pm 5{ }^{\circ} \mathrm{C}$. The photoperiod was set to $16 \mathrm{~h}$ using mercury vapour lamps (6600 lux at ear height). Relative humidity (RH) was $58 \%$. Greenhouse experiments were repeated twice, once in summer and once in winter. Dwarf maize was grown under the same greenhouse conditions as described for hybrid maize but also under controlled conditions in a climate chamber (Kaelte Klima, Northeim, Germany, $26^{\circ} \mathrm{C}, 70 \% \mathrm{RH}, 16 \mathrm{~h}$ photoperiod, 30000 lux $)$.

Maize seeds were sown in mixed soil consisting of $30 \%$ sand $(<2 \mathrm{~mm}$ granularity) and $70 \%$ commercial plant substrate (Fruhstorfer Erde, Typ T25, HAWITA Group, Vechta, Germany). After 21 days of growth, the hybrid maize plants were separated and single plants were transferred to plastic pots $(18 \times 18 \times 25 \mathrm{~cm})$. Dwarf maize seedlings were sown and raised in plastic pots $(11 \times 11 \times 12 \mathrm{~cm})$. Plants were irrigated with tap water as required. After one month of growth, plants were supplemented weekly with a macronutrient fertilizer $(\mathrm{N}: \mathrm{P}: \mathrm{K}=15: 10: 15)$, dissolved in irrigation water.

\subsubsection{Fungal Strains}

Infections of hybrid maize ears were carried out with two strains of $F$. graminearum (including a nivalenol (NIV) and a deoxynivalenol (DON) chemotype), two strains of $F$. verticillioides and one strain of $F$. subglutinans. Infection of dwarf maize ears included one strain of each $F$. graminearum, $F$. verticillioides and $F$. avenaceum. 
Apart from the strains that were used for maize infections, eight strains of $F$. subglutinans were used for the optimization of a species-specific real-time PCR assay for F. subglutinans. The sources of fungal material and abbreviations used in this study are described in Tab. 2.1.

Tab. 2.1 Overview of fungal material

\begin{tabular}{|c|c|c|c|}
\hline Fusarium sp. & Name & Source & $\begin{array}{l}\text { Abbreviation } \\
\text { used in the } \\
\text { present study }\end{array}$ \\
\hline \multirow[t]{2}{*}{ F. graminearum } & $\mathrm{Fg} 71^{\mathrm{a}}$ & T. Miedaner, University of Hohenheim, Germany & FG1 \\
\hline & $\mathrm{Fg} 210.1 \mathrm{wt}^{\mathrm{b}}$ & $\begin{array}{l}\text { Phytopathological strain collection, Division of Plant Pathology and } \\
\text { Crop Protection, University of Goettingen, Germany }\end{array}$ & $\mathrm{FG} 2$ \\
\hline \multirow[t]{2}{*}{ F. verticillioides } & Fv Ita 1 & A. Prodi, University of Bologna, Italy & FV1 \\
\hline & FM8114 & Fusarium Research Centre, Pennsylvania State University, USA & FV2 \\
\hline \multirow[t]{8}{*}{ F. subglutinans } & CBS 215.76 & CBS-KNAW Fungal Biodiversity Centre, Utrecht, The Netherlands & FSUB \\
\hline & 43.92 & H. Lew and A. Adler, Linz, Austria & \\
\hline & 215.96 & H. Lew and A. Adler, Linz, Austria & \\
\hline & Fsub2-17 & P. Karlovsky, Shaam 6-39, China & \\
\hline & Fsub2215 & E. Moeller, field isolate from maize, Radzików, Poland & \\
\hline & Fsub2213 & E. Moeller, field isolate from maize, Radzików, Poland & \\
\hline & Fsub2209 & E. Moeller, field isolate from maize, Radzików, Poland & \\
\hline & Fsub2220 & E. Moeller, field isolate from maize, Radzików, Poland & \\
\hline F. avenaceum & DSM 62161 & $\begin{array}{l}\text { Leibniz Institute - German Collection of Microorganisms and Cell } \\
\text { Cultures, Braunschweig, Germany }\end{array}$ & FA \\
\hline
\end{tabular}

\footnotetext{
${ }^{a}$ Nivalenol (NIV) chemotype

${ }^{\mathrm{b}}$ Deoxynivalenol (DON) chemotype
}

\subsubsection{Production of Fungal Spores}

Fungal spores were produced under sterile conditions in liquid mung bean medium following a modified protocol of Bai \& Shaner (1996). Modifications included a pre-cultivation step for 5 days at $28{ }^{\circ} \mathrm{C}$ to enhance final spore concentration. Final cultures were filtrated through cotton wool and centrifuged at $7320 \mathrm{~g}$ for $5 \mathrm{~min}$. The supernatant was discarded and spores 
were, subsequently, re-dissolved in glycerol (15\%, v/v, Carl Roth, Karlsruhe, Germany). Suspensions were mixed gently and the number of spores was counted with a Thoma chamber $\left(0.0025 \mathrm{~mm}^{2}\right)$. Spore suspensions were stored at $-70{ }^{\circ} \mathrm{C}$ and germination tests were regularly performed on potato-dextrose-agar (PDA, Carl Roth, Karlsruhe, Germany) to assess the viability of spores.

\subsubsection{Inoculation of Maize Ears}

Commercial hybrid maize ears were infected either with single fungal strains (FG1, FG2, FV1, FV2, FSUB) or with mixtures of $F$. graminearum strains and $F$. verticillioides strains. The mixed infection treatment MIX A included spores of FG1, mixed with FV1, whereas treatment MIX B included spores of FG2 and FV2. Experiments with dwarf maize included treatments with FG1, FV1 and FA (no mixtures).

Inoculum for the infection of maize ears was produced in autoclaved tap water. For single infection of hybrid maize ears, spore concentrations of $F$. verticillioides and F. subglutinans were set to $10^{6} \mathrm{~mL}^{-1}$. The spore concentration of $F$. graminearum (higher aggressiveness than other Fusarium spp. used in this study) was reduced to $10^{5} \mathrm{~mL}^{-1}$. Inoculum for mixed infection treatments was produced with a spore concentration of $10^{5} \mathrm{~mL}^{-1}$ for each species. Control plants were treated with water only. Each hybrid maize ear was treated with one $\mathrm{mL}$ inoculum by injecting the spore suspension into the silk-channel at main flowering stage (BBCH 65) following the inoculation method of Reid et al. (1995) (BBCH growth stages are based on the scale of Zadoks et al. (1974)).

For infections of dwarf maize ears, spore concentrations were set to $10^{5} \mathrm{~mL}^{-1}$ for $F$. graminearum and $2 \times 10^{5} \mathrm{~mL}^{-1}$ for $F$. verticillioides and $F$. avenaceum. Considering the small ear of dwarf maize, only $500 \mu \mathrm{L}$ inoculum per ear were used. In case of dwarf maize, $20 \mu \mathrm{L}$ of adhesion detergent Tween ${ }^{\circledR} 20$ (Applichem, Darmstadt, Germany) were added to each suspension. Control plants were treated with water and Tween ${ }^{\circledR} 20$.

Maturing stage of each plant was estimated daily and the date of infection (BBCH 65) was determined individually. Prepared inoculum was stored at $4{ }^{\circ} \mathrm{C}$ for no more than 3 days. Suspensions were kept on ice during inoculations and homogenized before use. Inoculations were carried out between 11 am and $2 \mathrm{pm}$. To avoid cross contaminations, separate syringes were used for each treatment. They were cleaned with ethanol $(80 \%$, v/v, Carl Roth, 
Karlsruhe, Germany), dried and reused. The number of replicates, harvest time point and further details about the experiments with hybrid and dwarf maize are given in Tab. 2.2. The success of infection and cross-contamination between treatments was estimated by quantitative real-time PCR (polymerase chain reaction, section 2.2.13).

\subsubsection{Handling of Plant Material for Chemical and Molecular Analysis}

Hybrid maize ears were harvested at 24 days past inoculation (dpi) $(n=5$, maturity stage of early milk ripeness, $\mathrm{BBCH} 73)$ as well as in time series at 6 different time points $(\mathrm{n}=3$, time points 4, 8, 12, 16, 20 and $24 \mathrm{dpi}$ ) (see Tab. 2.2). Dwarf maize $(\mathrm{n}=6)$ was already harvested at $14 \mathrm{dpi}$. Hybrid maize and dwarf maize ears were collected, dehusked and the evaluation of disease severity, as percentage of affected ear surface, was carried out on the naked ear. Afterwards, maize ears were cut longitudinally in two halves and handled according to the experimental design as illustrated in Fig. 2.1. For the analysis of volatiles, an aliquot of $2 \mathrm{~g}$ from the tip of one half were transferred to an inert glass vial for solid-phase microextraction (SPME) (20 mL volume, VWR, Darmstadt, Germany). The vial was sealed with a screw cap containing a silicone-polytetrafluoroethylene (PTFE) septum (1.3 mm, VWR, Darmstadt, Germany). Prior to use, the glass vial and metal screw cap had been pre-conditioned at $160^{\circ} \mathrm{C}$ for $3 \mathrm{~h}$ in order to remove any traces of volatile contaminants. Collected greenhouse samples in vials were immediately bedded in a closed ice bucket and stored at $-20{ }^{\circ} \mathrm{C}$ within $30 \mathrm{~min}$ from harvest (SPME collection in section 2.2.6). The other half of the ear was used for further analysis and stored at $-20{ }^{\circ} \mathrm{C}$ or $-70{ }^{\circ} \mathrm{C}$ in case of dwarf maize. Hybrid maize material from the second half was freeze-dried and ground with a ball mill (Retsch, Haan, Germany). Aliquots of homogenized material were used for quantitative real-time PCR (section 2.2.13) and for the analysis of mycotoxins and oxylipins (sections 2.2.12 and 2.2.15). Fresh dwarf maize material was processed in liquid nitrogen and used for quantitative real-time PCR, for gene expression analysis (section 2.2.14) and for the analysis of phytohormones and zealexins (section 2.2.16). Aliquots for each step of analysis are given in the corresponding sections below. 


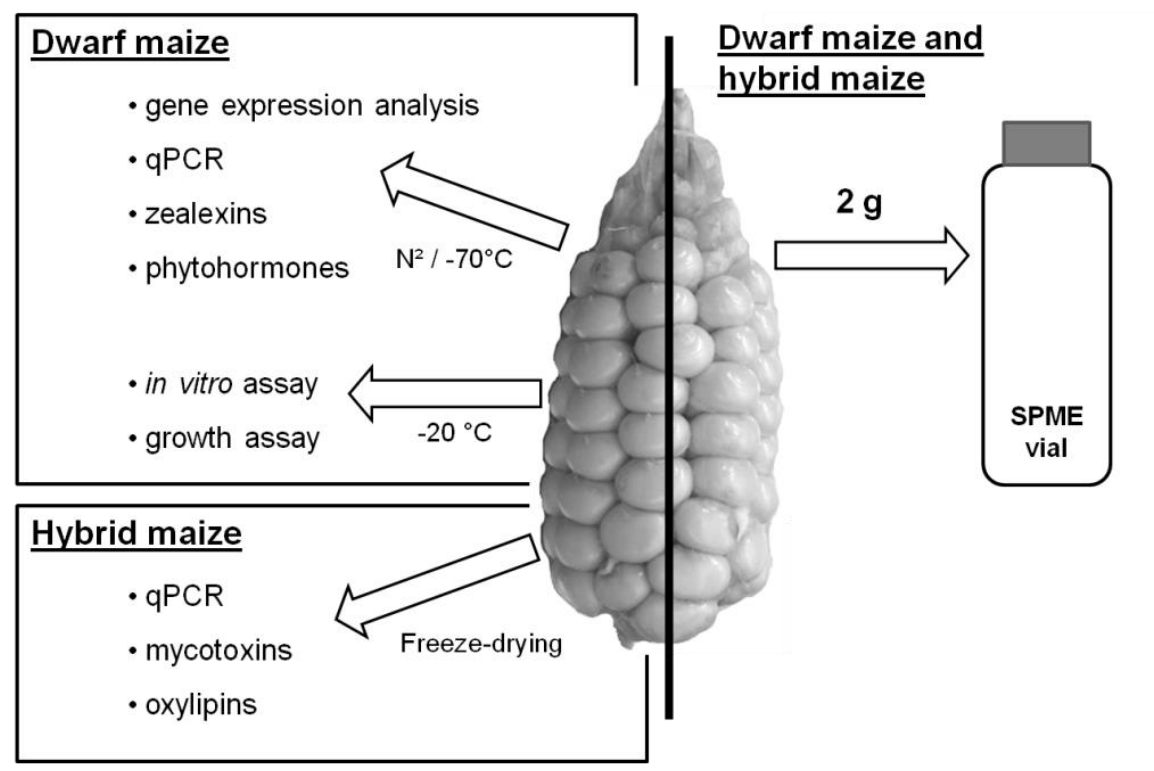

Fig. 2.1 Schematic display of the sample preparation of hybrid maize and dwarf maize ears after harvest: Collected ears were dehusked and cut in two halves: one half was used for (1) volatile profiling by solid-phase microextraction (SPME) followed by gas chromatography mass spectrometry (GC-MS) (SPME/GC-MS) and the other half of the ear was used for (2) supplementary analysis, such as determination of fungal DNA (quantitative real-time PCR, $\mathrm{PPCR}$ ) and transcription levels of genes involved in the formation of volatile compounds, as well as analysis of secondary metabolites (precise aliquots are given in the correspondent extraction protocols)

Furthermore, the systemic distribution of VOCs within different parts of the maize plant was monitored at 24 dpi in either three vertically divided parts of the hybrid maize ear (ear-tip, ear-centre, ear-base) or in three leaves of the plant (second leaf above maize cob, leaf next to the maize cob, second leaf below maize cob, see Tab. 2.2). Samples from maize ear material were collected as described earlier, considering the three different ear sections. Sampling of leaf material was carried out by separating a section of each leaf between $10 \mathrm{~cm}$ and $20 \mathrm{~cm}$ behind the stem. Afterwards, $1 \mathrm{~g}$ of the separated leaf material was coiled up, cut in four parts of the same size and transferred to an SPME glass vial. Samples were transported and stored as described before. 
Tab. 2.2 Experimental schedule for hybrid maize and dwarf maize

\begin{tabular}{|c|c|c|c|c|c|}
\hline \multirow[b]{2}{*}{ Treatment $^{1}$} & \multicolumn{4}{|c|}{ Hybrid maize } & \multirow{2}{*}{$\begin{array}{l}\text { Dwarf maize } \\
\text { SPME / GEA }\end{array}$} \\
\hline & $\begin{array}{c}\text { Static } \\
\text { sampling } \\
\left(\text { SPME }^{2}\right)\end{array}$ & $\begin{array}{c}\text { Dynamic } \\
\text { sampling } \\
\left(\mathrm{OLS}^{3}\right)\end{array}$ & Time series & $\begin{array}{l}\text { Systemic } \\
\text { Distribution }\end{array}$ & \\
\hline CONT (water control) & $\mathrm{n}=4$ & $\mathrm{n}=3$ & - & $\mathrm{n}=5$ & $\mathrm{n}=6$ \\
\hline FG1 & $\mathrm{n}=4$ & $\mathrm{n}=3$ & - & - & $n=6$ \\
\hline FG2 & $\mathrm{n}=4$ & $\mathrm{n}=3$ & 6 time points, $n=3$ & $\mathrm{n}=5$ & - \\
\hline FV1 & $\mathrm{n}=4$ & $\mathrm{n}=3$ & 6 time points, $n=3$ & $\mathrm{n}=5$ & $\mathrm{n}=6$ \\
\hline FV2 & $\mathrm{n}=4$ & $\mathrm{n}=3$ & - & - & - \\
\hline MIX A (FG1, FV1) & $\mathrm{n}=4$ & $\mathrm{n}=3$ & - & - & - \\
\hline MIX B (FG2, FV2) & $\mathrm{n}=4$ & $\mathrm{n}=3$ & - & - & - \\
\hline FSUB & $\mathrm{n}=4$ & $\mathrm{n}=3$ & - & - & - \\
\hline FA & - & - & - & - & $\mathrm{n}=6$ \\
\hline
\end{tabular}

1 Further descriptions and source of fungal material is given in Tab. 2.1

2 Solid-phase microextraction (SPME)

3 Open-loop stripping (OLS)

4 Gene expression analysis (GEA)

\subsubsection{Collection of Volatiles Using Static Procedure}

SPME was employed as static system for the collection of volatile molecules from the gas phase above plant material, the so-called headspace (HS). A SPME fibre, coated with polydimethylsiloxane divinylbenzene (PDMS-DVB, $65 \mu \mathrm{m}$ coating thickness, Supelco USA), was used as adsorption matrix. The fibre was initially cleaned by a bake-out at $260{ }^{\circ} \mathrm{C}$ for $60 \mathrm{~min}$, followed by three blank injections. Prior to volatile analysis, frozen maize samples in vials were allowed to equilibrate to room temperature and centrifuged at $1627 \mathrm{~g}$ for $2 \mathrm{~min}$ to collect maize material at the bottom of the glass vials. Prior to VOC extraction by SPME, samples were heated to $40{ }^{\circ} \mathrm{C}$ for $25 \mathrm{~min}$. The HS-SPME extraction was then carried out for $10 \mathrm{~min}$ at $40{ }^{\circ} \mathrm{C}$ (see Fig. 2.2). Equilibrium of extraction was obtained under these conditions. The fibre was immediately injected in the $\mathrm{GC}$ inlet (heated to $260{ }^{\circ} \mathrm{C}$ ) for VOC desorption and chromatographic analysis.

\subsubsection{Sampling of VOCs Using Dynamic Procedure}

An open-loop-stripping (OLS) system, with continuous supply of new ambient air, was applied on the live maize plant. The procedure is illustrated in Fig. 2.2. Glass cartridges, each packed with $1.5 \mathrm{mg}$ activated charcoal (AC) (obtained from D. Graenicher, Daumazan sur 
Arize, France), were used as volatile traps. Previous to use, these charcoal traps were rinsed 3 times with dichloromethane (DCM, anhydrous, $\geq 99.8 \%$, stabilized with amylene, SigmaAldrich, Taufkirchen, Germany) and 3 times with n-pentane (anhydrous, $\geq 99 \%$, SigmaAldrich, Taufkirchen, Germany). Traces of organic solvents were removed under vacuum (2 min). A gas-washing bottle $(250 \mathrm{~mL}$ total volume, $55 \mathrm{~mm}$ diameter, Carl Roth, Karlsruhe, Germany), filled with $100 \mathrm{~g}$ AC pellets (1 mm diameter, steam activated, Sigma-Aldrich, Taufkirchen, Germany), was used as a pre-filter for incoming ambient air. The head of the bottle was filled with glass wool to hinder leakage of AC pellets during the run. Commercial cooking bags, made of polyester (50 $\mathrm{cm}$ adjusted length, Toppits, Minden, Germany), were used to cover the maize ear. Silicon tubes $(4 \mathrm{~mm}$ inner diameter, Carl Roth, Karlsruhe, Germany) of a defined length (3 different sizes per set) were used to connect all system components (see also Fig. 2.2). Commercial polyvinylchloride tape (Coroplast, Wuppertal, Germany) was used to connect tubes and foil bag as tight as possible without harming the plant. The tape material had no contact with sample or headspace. A vacuum-pump, equipped with a PTFE-coated membrane (model N86KT.18, VWR, Darmstadt, Germany), was used to pull air through the system. A single pump was used for two OLS cycles at the same time via connection with Y-valve (polyprophylene, Carl Roth, Karlsruhe, Germany). The valve was placed behind the volatile traps. The flow rate in a single loop ranged between 1.1 and $1.5 \mathrm{~L}$ $\min ^{-1}$. OLS collections on maize ears were carried out for $24 \mathrm{~h}$ to exclude diurnal variations of volatile emission. The start of each collection was between 11 am and $4 \mathrm{pm}$. Blank runs were performed with empty bags to determine the background signal of the system.

After sampling, cartridges were rinsed with $100 \mu \mathrm{L} \mathrm{DCM}$ spiked with $8 \mu \mathrm{g} \mathrm{ml}^{-1}$ nonyl acetate (FCC grade, Sigma-Aldrich, Taufkirchen, Germany) as internal standard. The eluate was collected in $1.5 \mathrm{~mL}$ glass-vials, equipped with $200 \mu \mathrm{l}$ glass inserts (Macherey-Nagel, Dueren, Germany) and stored at $-20^{\circ} \mathrm{C}$. After Extraction, the volatile traps were cleaned with DCM and n-pentane as described before. Each trap was used up to 100 times. If necessary, VOC traps were rinsed with nitric acid (1 M, Sigma-Aldrich, Taufkirchen, Germany) followed by DCM and n-pentane clean-up. The AC containing gas-washing bottles, sampling bags and tubes were re-conditioned at $160^{\circ} \mathrm{C}$ for $3 \mathrm{~h}$. These components were used several times after re-conditioning. 
HS-SPME

\section{Open-Loop-Stripping}
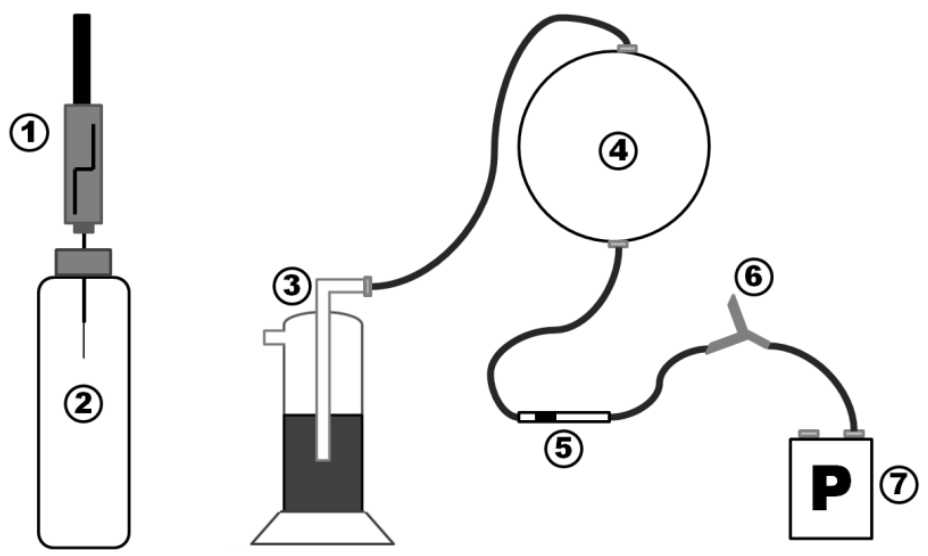

(1) SPME assembly

(2) Glass vial with sample

(3) Gas-washing bottle

(4) Sample containing bag

(5) Volatile trap

(6) Y-valve with second loop

(7) Vacuum pump

Fig. 2.2 Schematic display of the applied static (headspace solid-phase microextraction, HS-SPME) and dynamic (open-loop-stripping, OLS) sampling strategies for volatiles, air flow in OLS follows no. 3 (entry) to no. 7 (exit)

\subsubsection{Gas Chromatography (GC) Conditions for Volatile Profiling}

An Agilent 6890 gas chromatograph coupled with an Agilent 5973 mass selective detector (both obtained from Agilent, Waldbronn, Germany) and equipped with an autosampler for SPME vials (PAL Combi, obtained from CTC Analytics AG, Zwingen, Switzerland) was used to analyse the SPME samples and extracts of dynamic samplings. The GC was equipped with a capillary HP-5MS column (Agilent, Waldbronn, Germany, nominal diameter: $0.25 \mathrm{~mm}$, length: $30 \mathrm{~m}$, nominal film thickness: $0.25 \mu \mathrm{m}$ ). Conditions were as follows: inlet pressure: $48.5 \mathrm{kPa}$; He $19 \mathrm{~mL} \mathrm{~min}^{-1}$; injector: $250{ }^{\circ} \mathrm{C}$; transfer line: $260{ }^{\circ} \mathrm{C}$, electron energy: $70 \mathrm{eV}$. The $\mathrm{GC}$ was programmed as follows: $40{ }^{\circ} \mathrm{C}(2 \mathrm{~min}$ isothermic $)$, increasing at $3.2{ }^{\circ} \mathrm{C}$ $\min ^{-1}$ to $140{ }^{\circ} \mathrm{C}$ followed by $80{ }^{\circ} \mathrm{C} \min ^{-1}$ to $250{ }^{\circ} \mathrm{C}$ (3.38 min isothermic) and operated in splitless mode; carrier gas (He): $1 \mathrm{~mL} \mathrm{~min}^{-1}$. The scan range was between 40 and 450 atomic mass units. OLS samples were injected with a volume of $2 \mu \mathrm{L}$. For liquid injections, the syringe was washed two times with DCM after each injection. Furthermore, a GC with flame ionization detector (FID, GC model 6890n, Agilent, Waldbronn, Germany) was employed to analyse OLS extracts for quantitative purposes. Data processing is described in section 2.2.18. 


\subsubsection{Comparing Static and Dynamic Sampling}

Infected kernels from a hybrid maize ear, treated with F. graminearum spores (strain FG1), were collected after $24 \mathrm{dpi}$ and homogenized with mortar and pestle. Aliquots of $5 \mathrm{~g}$ fresh matter were distributed in 3 replicates to SPME vials and glass Petri dishes (92 mm diameter) (vessels were preconditioned at $160{ }^{\circ} \mathrm{C}$ for $3 \mathrm{~h}$ prior to use). The HS-SPME was conducted as described before (section 2.2.6). For OLS, the glass Petri dishes with infected material were placed in foil bags and connected to a dynamic system as described earlier (section 2.2.7). The OLS was carried out for $4 \mathrm{~h}$. An empty bag was looped as control. Nonyl acetate $(8 \mu \mathrm{g}$ $\mathrm{ml}^{-1}$ ) was used as an internal standard in the OLS procedure. Both, HS-SPME and OLS, were carried out at the same time to exclude differences in VOC signatures due to decomposition of material. GC-MS analysis was carried out as described earlier (section 2.2.8).

\subsubsection{In vitro Assay with Fusarium Cultures on Autoclaved Maize and Rice Kernels}

$F$. graminearum strain 1 (FG1) and $F$. verticillioides strain 2 (FV2) were incubated for 9 days on GM7 medium (Utermark \& Karlovsky, 2007) $\left(25^{\circ} \mathrm{C}\right.$, dark conditions). Aliquots of $3 \mathrm{~g}$ non-infected maize material, harvested in greenhouse trials, were transferred to SPME glass vials. Relative water content was adjusted to $80 \%(\mathrm{w} / \mathrm{w})$ by addition of double distilled water. The equivalent weight of dry rice kernels (not contaminated, BAK Kardeşler GmbH, Gemlik, Turkey) were transferred to glass vials and relative water content was adjusted to $80 \%(\mathrm{w} / \mathrm{w})$ as well. Vials were autoclaved at $121^{\circ} \mathrm{C}$ for $20 \mathrm{~min}$ and cooled down to room temperature. Overgrown GM7-plugs ( $\varnothing 0.5 \mathrm{~mm}$ ) with young fungal mycelium of FG1 and FV2 were separated with a cork borer and transferred to the prepared vials. A sterile GM7-plug was transferred to autoclaved medium as negative control. Prepared cultures $(n=3)$ were incubated for 7 days in darkness at room temperature. During the first 5 days, vials were not sealed airtight to allow gas exchange. To avoid contamination, they were sealed with one layer of Parafilm ${ }^{\circledR}$ M (American National Can, Greenwich, Connecticut, USA). Vials were sealed airtight during the last $48 \mathrm{~h}$ prior to analysis to allow the accumulation of volatiles in the headspace. Gas chromatographical analysis was carried out by SPME/GC-MS as described earlier (section 2.2.8). 


\subsubsection{Effect of VOCs from Infected Ear Material on Fungal Growth}

$F$. graminearum strain FG1 and $F$. verticillioides strain FV1 were incubated on PDA for 3 days $\left(25^{\circ} \mathrm{C}\right.$, darkness). Aliquots of $2 \mathrm{~g}$ maize material from greenhouse trials (control treatment, $F$. verticillioides (FV1) and F. graminearum (FG2) treatment) were blended with $8 \mathrm{~mL}$ DCM. Extracts were shaken for $10 \mathrm{~h}$ and centrifuged for $10 \mathrm{~min}$ at $7320 \mathrm{~g}$. Supernatant was transferred to a new centrifuge tube (Sarstedt, Nuembrecht, Germany) and the total volume was filtered though a PTFE-filter (Perfect Flow, $13 \mathrm{~mm}, 0.2 \mu \mathrm{m}$, Wicom, Heppenheim, Germany). Extracts were stored in glass vials at $-20{ }^{\circ} \mathrm{C}$. PDA medium was poured out in one-half of sterile two-chamber Petri dishes $(90 \mathrm{~mm}$ diameter, Sarstedt, Nuembrecht, Germany). The other half was filled with a layer of sterile cotton wool. An overgrown agar plug with young fungal mycelium of each Fusarium strain ( $5 \mathrm{~mm}$ diameter) was transferred to the centre of each PDA half. Fungal material was incubated at $25^{\circ} \mathrm{C}$ in darkness. After $24 \mathrm{~h}$, the Petri dishes were sealed with one layer of PTFE tape (Wendler, Aue, Germany) and one layer commercial PVC tape. A loophole was gently melted in the lid above the cotton wool using a hot injection needle. Aliquots of $40 \mu \mathrm{L}$ DCM extracts were transferred to the cotton wool. Each growing strain was treated with (1) pure DCM, (2) extracts of control plant material and with (3) extracts of material infected with the same strain $(n=3)$. The hole was immediately closed with a PTFE coated septum. The diameter of the growing culture was measured 3 times within $72 \mathrm{~h}$.

\subsubsection{Mycotoxin Extraction and Analysis}

Mycotoxin content was determined by HPLC-MS using methods described before for thrichothecenes DON and NIV (Adejumo et al., 2007a), zearalenone (ZEN) (Adejumo et al., 2007b) and Fumonisins B1 and B2 (further labelled as FB1, FB2) (Visentin et al., 2012).

\subsubsection{Quantification of Fungal DNA}

DNA was extracted from aliquots of $100 \mathrm{mg}$ maize ear material following a protocol of Brandfass \& Karlovsky (2008). Gel electrophoresis was performed to confirm the quality and uniformity of extraction process. DNA was loaded on $0.8 \%(\mathrm{w} / \mathrm{v})$ agarose gel prepared in TAE buffer (40mM Tris, $1 \mathrm{mM}$ EDTA (ethylenediaminetetraacetic acid) (both substances were obtained from Carl Roth, Karlsruhe, Germany), $\mathrm{pH}$ adjusted to 8.5). Electrophoresis was




Applichem, Darmstadt, Germany) and documented with a gel imaging system (Vilber Lourmat, Marne la Vallee, France). To reduce content of inhibitory TE buffer, DNA was diluted 1:10 (v/v) with double distilled water.

Low-volume quantitative PCR assays were developed by Dastjerdi et al. (unpublished). Reaction mixtures contained double distilled water, filled to $4 \mu \mathrm{L}$ reaction volume per well, $\mathrm{NH} 4$-reaction buffer $\left(16 \mathrm{mM}\left(\mathrm{NH}_{4}\right)_{2} \mathrm{SO}_{4}\right.$ and $67 \mathrm{mM}$ Tris-HCI), species specific primers $(0.3 \mu \mathrm{M}$ forward (fwd) and reverse (rev) primer), $0.1 \times$ SYBR Green I solution (Invitrogen, Karlsruhe, Germany), BIOTaq DNA polymerase, $\mathrm{MgCl}_{2}$ buffer, dNTP solution and $1 \mu \mathrm{L}$ sample template (concentration for reagents, i.e. polymerase and buffers, is presented in Tab. 2.3). Buffers, dNTPs and Taq polymerase were obtained from Bioline (Luckenwalde, Germany). Quantitative real-time PCR analysis was performed with a CFX 800 real-time PCR cycler (BioRad, Hercules, California, USA). Melt curve analysis was included to confirm the specifity of individual PCR product. Wells with unspecific fluorescence signals, i.e. due to primer dimers, were not quantified. An overview of applied PCR procedures is presented in Tab. 2.3 .

Tab. 2.3 Characteristics of PCR assays for the quantification of fungal DNA in maize samples

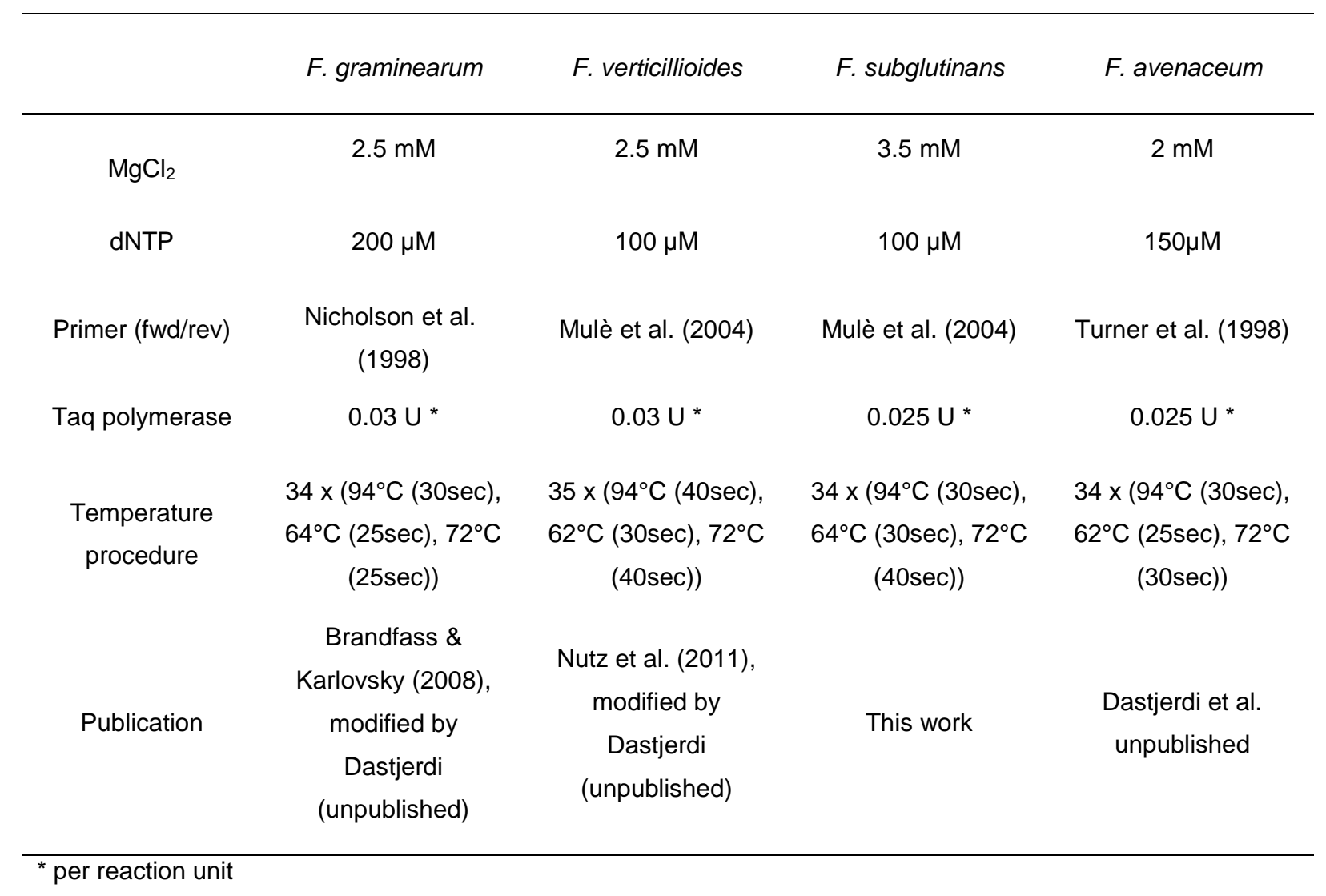


Fluorescence signals were exported and factorized as described in Brandfass \& Karlovsky (2008). Fungal DNA concentrations in unknown samples were calculated according to a standard row (8 DNA concentration steps from $0.51 \mathrm{pg}^{-1}$ to

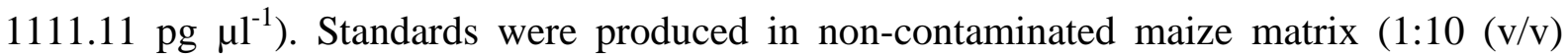
diluted in double distilled water), which was spiked as required with concentrated fungal DNA. To obtain fungal DNA in high concentrations, Fusarium strains were cultivated in potato dextrose broth (PDB, Carl Roth, Karlsruhe, Germany) for 5 to 7 days at $26{ }^{\circ} \mathrm{C}$. Fungal mycelium was collected and freeze-dried. The DNA extraction from homogenized fungal material was conducted as described in Brandfass \& Karlovsky (2008), but included an additional phenol clean-up step to remove impurities. The DNA concentration of standard stock solutions was determined by densitometrical analysis on $1.2 \%(\mathrm{w} / \mathrm{v})$ agarose gel in TAE buffer (60V, 60min) using Multi Analyst software (BioRad, Hercules, California, USA).

\subsubsection{Analysis of Terpene Synthase Genes tps6/11}

Total RNA was extracted from aliquots of $100 \mathrm{mg}$ fresh matter (ground with mortar and pestle in liquid nitrogen) using RNeasy Plant Mini Kit (Quiagen, Hilden, Germany). Quality of extraction was confirmed by gel electrophoresis (1.2\% (w/v) agarose gels).

Further analysis was carried out in the Max Planck Institute for Chemical Ecology in Jena (Germany) (gently guided by Dr. T. Koellner and S. Irmisch). Concentration of RNA was measured with a NanoDrop 2000 spectrophotometer (Thermo Fisher, Wilmington, DE, USA)

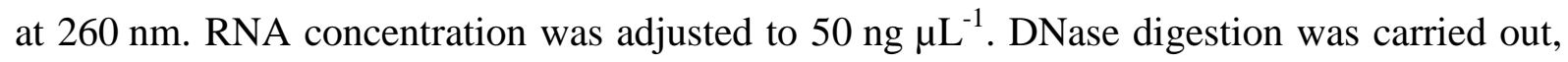
followed by synthesis of cDNA using Super Script III polymerase (Invitrogen, Karlsruhe, Germany). Each volume was diluted 2:1 (v/v). Amplification of DNA was accomplished for each candidate primer pair using Phusion High-Fidelity DNA-Polymerase (Biozym, Hessisch Oldendorf, Germany). Primer pairs were pre-selected on the basis of gel electrophoresis. PCR products were purified with QIAquick PCR Purification KIT (Quiagen, Hilden, Germany). Ligation of DNA fragments in $\mathrm{pCR}^{\circledR}$ Blunt II-TOPO ${ }^{\circledR}$ vector (Invitrogen, Karlsruhe, Germany) followed according to the manufacturers' instructions. The vector was transformed in competent cells of Escherichia coli. Colony PCR was carried out to identify successful transformation. After an incubation period of $24 \mathrm{~h}$ at $37^{\circ} \mathrm{C}$, Plasmid DNA was extracted from E. coli cultures with NucleoSpin Plasmid Kit (Macherey-Nagel, Dueren, Germany). Plasmid inserts were sequenced to confirm correct transformation. Quantitative real-time PCR was carried out for housekeeping genes (putative APT1a for adenine phosphoribosyltransferase 1; 
primer forward HG5: 5' -AGGCGTTCCGTGACACCATC-3', primer reverse HG6: 5' - CTGGCAACTTCTTCGGCTTCC-3') and candidate genes (tps6/11, QRT5: 5'TGGTAATTTGGACTTAGTTTCACG-3'; QRT6: 5' - CACTGTCTCTCCATGAATCCTC3') using Brilliant III Ultra-Fast qPCR master mix (Agilent, Waldbronn, Germany). Relative fold change of target genes in comparison to housekeeping genes was calculated for each treatment.

\subsubsection{Detection of Oxylipins}

The formation of oxylipins was analysed by Dr. C. Herrfurth and Prof. I. Feussner in the Department for Plant Biochemistry, Albrecht-Haller-Institute for Plant Sciences (Goettingen, Germany). Aliquots of freeze-dried hybrid maize material, corresponding to $2 \mathrm{~g}$ fresh weight, were extracted according to the protocol of Goebel et al. (2003) and methylated with trimethylsilyldiazomethane (2 M in hexane, Sigma-Aldrich, Taufkirchen, Germany). The analysis of non-oxidized fatty acid methyl esters (FAMES) was performed with an Agilent 6890 gas chromatograph equipped with a capillary DB-23 column (Agilent, Waldbronn, Germany, nominal diameter: $0.25 \mathrm{~mm}$, length: $30 \mathrm{~m}$, nominal film thickness: $0.25 \mu \mathrm{m}$ ) and heptadecanoic acid as internal standard. Hydroxyl fatty acids were purified on reverse phaseHPLC equipped with ET250/2 Nucleosil 120-5 C18 column (Macherey-Nagel, Dueren, Germany) as described in Goebel et al. (2003). Eluate fraction was collected between 8 and $13.5 \mathrm{~min}$, evaporated to dryness and re-dissolved in $2 \mu \mathrm{L}$ acetonitrile. After addition of $2 \mu \mathrm{L}$ N,O-bis(trimethylsilyl)trifluoroacetamide (Sigma-Aldrich, Taufkirchen, Germany), analysis was carried out with an Agilent 6890 gas chromatograph equipped with DB-23 column and coupled with an Agilent 5973 MS. Standard curves were constructed by plotting ion intensities vs. molar amounts of known hydroxyl fatty acids.

\subsubsection{Extraction and Analysis of Zealexins and Jasmonic Acid (JA)}

Aliquots of $100 \mathrm{mg}$ fresh dwarf maize ear material were transferred to $2 \mathrm{~mL}$ reaction tubes. A volume of $800 \mu \mathrm{L}$ 1-propanol- $\mathrm{H}_{2} \mathrm{O}-\mathrm{HCL}$ (2:1:0.005, v/v/v, fuming HCL, 37\%, Merck, Darmstadt, Germany), containing deuterium labelled jasmonic acid (d5-JA, $0.15 \mu \mathrm{g} \mathrm{ml}^{-1}$, Dr. Ehrenstorfer, Augsburg, Germany) as internal standard, was added to each tube (extraction steps were performed in darkness). After $60 \mathrm{~min}$ of incubation on ice, samples were shaken for $30 \mathrm{~min}$. Aliquots of $1 \mathrm{~mL}$ cold DCM were added and samples were shaken again for $15 \mathrm{~min}$ on ice. Extracts were centrifuged for $30 \mathrm{~s}$ at $10000 \mathrm{~g}$ and the bottom layer (DCM-1- 
propanol phase) was transferred into $4 \mathrm{~mL}$ glass vials. Two aliquots of $400 \mu \mathrm{L}$ were collected from each sample. One of the aliquots was used for phytohormone analysis and the other one for the analysis of zealexins.

The phytohormone analysis was carried out by Dr. R. Splivallo (Molecular Phytopathology and Mycotoxin Research, University of Goettingen, Germany). For this purpose, samples were evaporated to dryness under vacuum, stored and re-dissolved in $200 \mu \mathrm{L}$ methanol-water $(1: 1, \mathrm{v} / \mathrm{v})$ containing $7 \mathrm{mM}$ acetic acid (Sigma-Aldrich, Taufkirchen, Germany) for HPLCMS analysis. Aliquots of $10 \mu \mathrm{L}$ were injected to HPLC and eluted at $40{ }^{\circ} \mathrm{C}$ with a flow rate of $0.2 \mathrm{~mL} \mathrm{~min}^{-1}$. Chromatography was performed on a Kinetex ${ }^{\circledR} \mathrm{C} 18$ column $(100 \mathrm{~mm} \times 2.10 \mathrm{~mm}$ with $2.6 \mu \mathrm{m}$ particle size) equipped with a C18 guard column $(2.0 \mathrm{~mm})$ purchased from Phenomex Inc. (Aschaffenburg, Germany). The following program was used for elution: $90 \%(\mathrm{v} / \mathrm{v})$ solvent A ([95:5] [ $\mathrm{H}_{2} 0$ :Acetonitrile] containing $7 \mathrm{mM}$ acetic acid) and $10 \%(\mathrm{v} / \mathrm{v})$ solvent $\mathrm{B}$ (methanol containing $7 \mathrm{mM}$ acetic acid) for $1 \mathrm{~min}$; ramp to $20 \%(\mathrm{v} / \mathrm{v}) \mathrm{B}$ in $3 \mathrm{~min}$ $19 \mathrm{sec}$, ramp to $30 \%(\mathrm{v} / \mathrm{v}) \mathrm{B}$ in $36 \mathrm{sec}$; ramp to $98 \%(\mathrm{v} / \mathrm{v}) \mathrm{B}$ in $1 \mathrm{~min}$; hold for $2 \mathrm{~min} 32 \mathrm{~s}$ followed by re-equilibration to $10 \% \mathrm{~B}$. Under those conditions, JA eluted at $7.4 \mathrm{~min}$. Phytohormones were detected in the mass spectrometer in multiple reaction monitoring modus (MRM) with the following mass transitions: JA, $208.9>59.0$ (9.5 eV); d5-JA, $214>61.8(14.0 \mathrm{eV})$. A calibration curve based on the ratio of the peak areas of unlabeled JA standard to d5-JA standard was used for the quantification of JA.

For zealexin analysis, samples were derivatized with $4 \mu$ mol trimethylsilyldiazomethane ( $2 \mu \mathrm{L}$ of a $2 \mathrm{M}$ solution in hexane), as described in Schmelz et al. (2004). Samples were injected with a volume of $1 \mu \mathrm{L}$ to an Agilent $6890 \mathrm{GC}$, equipped with a DB-23 column, coupled with an Agilent 5973 MS. Conditions were as follows: inlet pressure: $52.5 \mathrm{kPa}$; He $11 \mathrm{~mL} \min ^{-1}$; injector: $260{ }^{\circ} \mathrm{C}$; transfer line: $260{ }^{\circ} \mathrm{C}$, electron energy: $70 \mathrm{eV}$. The $\mathrm{GC}$ was programmed as follows: $50{ }^{\circ} \mathrm{C}\left(1 \mathrm{~min}\right.$ isothermic), increasing at $10.0{ }^{\circ} \mathrm{C} \min ^{-1}$ to $260{ }^{\circ} \mathrm{C}$ (15 min isothermic) and operated in split mode (split ratio of 7:1); carrier gas (He): $1 \mathrm{~mL} \mathrm{~min}^{-1}$. Data processing included integration of chromatograms and identification by mass-spectral data (refer to section 2.2.18). 


\subsubsection{Stem Elicitation Assays}

A stem phytoalexin elicitation assay was carried out according to the descriptions of Huffaker et al. (2011). Stock solutions of JA and Ethephon (ETP) were prepared in ethanol (PA grade) (Sigma-Aldrich, Taufkirchen, Germany). Working solutions were prepared in double distilled water (ethanol $5 \%$, v/v; (1) 200 mmol JA (as $\mathrm{Na}^{+}$salt) and (2) $66 \mathrm{nmol}$ ETP in $20 \mu \mathrm{L}$ volume, (3) mix of $200 \mathrm{nmol} \mathrm{JA}$ and 66nmol ETP (JA-ETP) in $40 \mu \mathrm{l}$ volume). Five week old dwarf maize plants, cultivated in greenhouse as described earlier (section 2.2.1), were cut off $5 \mathrm{~cm}$ above soil. Two sections of $3 \mathrm{~cm}$ were separated from the lower stem part of each plant. A defined incision of $2 \mathrm{~mm}$ depth was cut in each section at full length. Prepared solutions of JA, ETP and JA-ETP were transferred to the incisions. Aliquots of $20 \mu \mathrm{L}$ ethanol $5 \%(\mathrm{v} / \mathrm{v})$ were used as control treatment $(\mathrm{n}=3)$. Inoculated stem sections were embedded in $20 \mathrm{~mL}$ inert glass vials and incubated for $24 \mathrm{~h}$ at room temperature and normal light conditions. After incubation, samples were stored at $-20^{\circ} \mathrm{C}$. HS-SPME sampling and GC-MS analysis were carried out as described earlier (section 2.2.8).

Stems of healthy dwarf maize plants were treated following the descriptions of Huffaker et al. (2011). For this purpose, stems were incised with a scalpel $2 \mathrm{~cm}$ above the soil. Incisions were filled gently with $20 \mu 1100 \mathrm{~nm}$ JA and $33 \mathrm{~nm}$ ETP (dissolved in a $5 \%(\mathrm{v} / \mathrm{v}$ ) ethanol solution) or with $20 \mu \mathrm{l}$ ethanol $5 \%$ (v/v) as control treatment. After $24 \mathrm{~h}$ of incubation, stem sections with incisions were collected and transferred to SPME vials. Samples were analyzed by SPME/GC-MS as described in the previous section (2.2.8).

\subsubsection{Data Processing and Statistical Analysis}

Chromatograms of OLS and SPME samples were integrated automatically using Agilent MSD ChemStation software (version D.01.02.16, 15 June 2004, Agilent, Waldbronn, Germany). Integration parameters were set to: initial area reject $=1$; initial peak width $=0.02$; shoulder detection $=\mathrm{OFF}$; initial threshold $=14$. Kováts' retention indices $(\mathrm{KI})$ were calculated based on a homologous series of $n$-alkanes (Alencar et al., 1984).

Species-specific real-time PCR was used to validate fungal infections. Samples that were infected with other Fusarium sp. than the intended species were removed from analysis. 
To allow a statistical data evaluation for both sampling strategies (SPME/GC-MS and OLS/GC-MS), retention times were aligned manually to one decimal place. The alignment of retention times was justified with respect to the high resolution and low temporal shift among peaks. A matrix was created, in which the peak areas of each sample were assigned to the corresponding retention times. Accordingly, the designed data matrix included all retention times and assigned peak areas occurring in one experiment. In cases were chromatograms revealed no integrable areas for specific retention times, the peak areas were set to zero.

Prior to statistical analysis, no data normalization was performed for the SPME/GC-MS data, while OLS/GC-MS peak areas were normalized to the peak area of the internal standard.

An R-script (version 2.14.2011-12-16 for Windows, http://www.r-project.org/) was applied for further data processing. The non-parametric Kruskal-Wallis test (one-way analysis of variance by ranks), as described in Siegel \& Castellan (1988), was employed to identify significant differences between control and individual Fusarium infection treatments. The formula is given below:

$$
\begin{array}{ll}
\left|\bar{R}_{c}-\bar{R}_{u}\right| \geq z_{\alpha / 2(k-1)} \sqrt{\frac{N(N+1)}{12}} \times\left(\frac{1}{n_{c}}+\frac{1}{n_{u}}\right) \\
\bar{R} & =\text { average of ranks within group } \\
c & =\text { control group } \\
u & =\text { group (treatment, other than control) } \\
k & =\text { number of groups (treatments) } \\
\mathrm{N} & =\text { number of cases in the combined sample } \\
n & =\text { number of cases within group }
\end{array}
$$

The Kruskal-Wallis test is included in the contributing package 'pgirmess: data analysis in ecology' (version 2.14.2, recent version is available at http://cran.rproject.org/web/packages/pgirmess/index.html). The test was conducted on each retention time vector containing peak areas of all replicates within treatments (= groups). These vectors correspond to individual volatile compounds. Vectors that revealed significant differences between one or more infection treatments and the healthy control were considered as markers.

Marker identification was performed by calculation of retention indices (KI), by injection of authentic standards (only major isoprenes) and by comparison of their MS fragmentation pattern to those available in mass spectral libraries, including NIST 08 (National institute of Standards and Technology, Gaithersburg, Maryland, USA), the Adams Library (Robert P. 
Adams, 2007, Identification of Essential Oil Components by Gas Chromatography/ Mass Spectrometry, Allured Publishing Corporation, Carol Stream, Illinois, USA) and the Wiley Registry of Mass Spectral Data ( $8^{\text {th }}$ edition, John Wiley \& Sons Inc., Hoboken, New Jersey, USA).

Quantitative data, i.e. for toxin concentration, fungal biomass and oxylipins (quantification by standard rows), were averaged within treatments. Results below LOQ were set to 0. Multiple comparisons were carried out in a post-hoc Scheffé test $(p<0.05)$ for data sets with unequal sample size and Tukey's honestly significant difference test $(\mathrm{p}<0.05)$ for balanced data sets following ANOVA. 


\subsection{Results}

\subsubsection{Optimization of a Specific Real-Time PCR Assay for F. subglutinans}

Three published primer pairs were evaluated for the optimization of a specific real-time PCR assay for F. subglutinans (Primer pair 1: Fsub565 fwd (5'-TCATTGGTA TGTTGTCGCTCATG - 3'), Fsub622A rev (5'- GTGATATGTTAGTACGAATAAAGGGA GAAC-3'), published by Nicolaisen et al. (2009), Primer pair 2: 61-2 F (5'- GGCCACTCAAGAGGCGAAAG - 3'), 61-2 R (5' - GTCAGACCAGAGCAAT GGGC - 3'), published by Moeller et al. (1999), Primer pair 3: SUB 1 (5'-CTGT CGCTAACCTCTTTATCCA - 3'), SUB 2 (5'-CAGTATGGACGTTGGTATTATAT CTAA - 3'), published by Mulè et al. (2004)). In a first step, preliminary primer specifity tests were performed using pure standard DNA of eight $F$. subglutinans isolates (No. 43.92, No. 215.96 (H. Lew and A. Adler, Linz, Austria), Fsub2-17 (P. Karlovsky, Shaam 6-39, China), Fsub2215, Fsub2213, Fsub2209, Fsub2220 (E. Moeller, field isolates from maize, Radzików, Poland) and FSUB (CBS 215.76, full reference in section 2.2.2). Furthermore, we included F. graminearum strain 1 (FG1) and F. verticillioides strain 1 (FV1) as reference DNA for non-target sequences. A standardized PCR protocol was established according to the published optimal conditions of all three primer pairs $\left(2 \mathrm{mM} \mathrm{MgCl}_{2}, 200 \mu \mathrm{M} \mathrm{dNTP}\right.$, annealing temperature: $62{ }^{\circ} \mathrm{C}$ for $60 \mathrm{sec}$ ). Preliminary PCR assays, including melt curve analysis, were carried out with a BioRad iCycler (BioRad, Hercules, California, USA). Primer pair 1 resulted in unspecific PCR products for water control, F. subglutinans DNA and reference DNA and was, consequently, excluded from further analysis. Primer pair 2 and 3 amplified F. subglutinans DNA with the same efficiency, but primer pair 2 did not specifically amplify target DNA. Consequently, further PCR optimization was carried out with primer pair 3 and DNA extracted from the described FSUB strain. Gradient real-time PCR using CFX 800 realtime PCR cycler was carried out for an annealing temperature range between $54{ }^{\circ} \mathrm{C}$ and $68{ }^{\circ} \mathrm{C} . \mathrm{MgCl}_{2}$ concentrations were tested in the range of $2.0-4.5 \mathrm{mM}$ and applied dNTP concentrations ranged between $100-250 \mu \mathrm{M}$. Considering ct-values and specifity of primer annealing, it can be recommended to use a $\mathrm{MgCl}_{2}$ concentration of $3.5 \mathrm{mM}$ and a dNTP concentration of $100 \mu \mathrm{M}$. The PCR assay can be operated in a broad temperature range. Nevertheless, $64-66{ }^{\circ} \mathrm{C}$ turned out to be most suitable to achieve low ct-values and specifity of primer annealing. 


\subsubsection{Comparison of Dynamic and Static Sampling Strategies for the Collection of VOCs}

SPME is a non-exhaustive equilibrium based extraction technique, which permits quick extraction of volatiles from complex matrices without time consuming sample preparation (Ouyang \& Pawliszyn, 2008; Vas \& Vékey, 2004). SPME is considered to be at best semiquantitative without the use of proper calibration accounting for possible matrix effects. For SPME extractions, we not only normalized sample mass (always $2 \mathrm{~g}$ ), extraction time (10 min) and temperature $\left(40{ }^{\circ} \mathrm{C}\right)$, but also compared the results of SPME with OLS, an exhaustive extraction technique for which we used an internal standard for data normalization. Comparison of the results, obtained with both sampling methods, indicates a high correlation $\left(\mathrm{R}^{2}=0.998\right)$ between OLS data, normalized to the internal standard, and the non-normalized SPME data (see Fig. 2.3). This illustrates that matrix effects are negligible during SPME sampling. It also implies that our SPME data, from maize ear samples, yield quantitative results, which permits to directly compare a given volatile peak area, from one sample to the other.



Fig. 2.3 Comparison of dynamic (openloop-stripping, OLS) and static (SPME) sampling strategies reveals strong correlation between both sampling types, $\left(R^{2}=0.998\right)$, comparison was carried out for 5 sesquiterpenoid compounds, emitted by F. graminearum infected maize kernels, TIC $=$ total ion chromatogram, data presented as means \pm standard deviation, $\mathrm{n}=3$ 


\subsubsection{Set of Volatile Biomarkers for Fusarium Infection of Maize Ears by SPME/GC- MS Analysis}

The volatile profile of Fusarium infected hybrid maize ears differed considerably from the profile of non-infected maize. Statistically significant differences $(\mathrm{p}<0.01)$ between non-infected and infected maize ears were observed for a set of 27 volatile compounds (infection markers), detected at $24 \mathrm{dpi}$ (Tab. 2.4 and Fig. 2.4 A). We observed de novo synthesis of VOCs upon infection as well as a relative increase or decrease in VOC emission. This illustrates qualitative (absence or presence) and quantitative differences. Depending on their occurrence, markers were specific to single species of Fusarium (i.e. trichodiene for $F$. graminearum infection) or general of Fusarium infection (i.e. $\beta$-selinene occurred in infected ear material independent from the species). The set of markers contained alcohols and carbonyls $\left(\mathrm{C}_{5}-\mathrm{C}_{8}\right.$ compounds), as well as sesquiterpenoid compounds $\left(\mathrm{C}_{15}\right.$, SQTs).

A large group of volatile terpenoid markers was found in infected hybrid maize. Among this group, 5 sesquiterpenes, $\beta$-selinene, $\alpha$-selinene, $\beta$-macrocarpene, $\beta$-bisabolene and trichodiene (only in FG1/FG2), were identified as main markers, because this group appeared only upon infection and peak abundances were remarkably higher than for other volatile signals. Fig. 2.5 presents a stacked chromatogram of a control and a $F$. graminearum treatment, illustrating the formation of these main VOC markers upon infection. The infection with $F$. graminearum resulted in a diverse set of other isoprenoid markers, including (+)-aromadendrene, longifolene, germacrene $\mathrm{D}, \quad(\mathrm{E})-\beta$-farnesene, $\alpha$-muurolene, but also unidentified sesquiterpenoids. The mass spectra of unknown SQTs, extracted from chromatograms of hybrid maize samples, infected with $F$. graminearum (strain FG1), are presented in Fig. 2.7. The unknown SQT (KI 1418), germacrene D and $\alpha$-muurolene were also released in all other infection treatments but not considered as markers for these treatments, whereas (E)- $\beta$-farnesene and two unidentified SQTs (KI 1488 and KI 1547) were also present in F. subglutinans infected ears (in this case as well no significant differences observed). Non-infected hybrid maize ears released (+)-cycloisosativene and $\alpha$-ylangene at highest levels. These volatile SQTs were absent or found with minor abundances in the headspace of Fusarium infected samples. 
Tab. 2.4 Overview of volatile markers for Fusarium infection of hybrid maize ears and statistical differences to control treatment at time point 24 days post-inoculation (dpi) (analysis by SPME/GC-MS)

\begin{tabular}{|c|c|c|c|c|c|c|c|c|c|c|}
\hline \multirow{2}{*}{$\mathrm{KI}_{\text {calc }}^{1}$} & \multirow{2}{*}{ Chemical identity } & \multirow{2}{*}{$\begin{array}{c}\text { Mode of } \\
\text { identification }^{2}\end{array}$} & \multirow{2}{*}{$\mathrm{OLS}^{3}$} & \multicolumn{7}{|c|}{ Observed differences ${ }^{4}$ between infected ${ }^{5}$ and non-infected ${ }^{6}$} \\
\hline & & & & $\mathrm{FG} 1$ & FG2 & FSUB & FV1 & FV2 & MIXA & MIXB \\
\hline 753 & 1-pentanol & $\mathrm{KI}, \mathrm{MS}$ & & 2.5 & 0 & 4.8 & $12.8^{*}$ & 0.8 & 0.5 & 1.5 \\
\hline 856 & 3-hexen-1-ol & $\mathrm{KI}, \mathrm{MS}$ & & $11.4^{*}$ & $11.4^{*}$ & 3 & 1.4 & 7.5 & 11.4 & 11.4 * \\
\hline 889 & no ID & & & 7.1 & 11.4 * & 3.1 & 1.8 & 4.8 & 8.4 & 2.6 \\
\hline 904 & 2-heptanol & $\mathrm{KI}, \mathrm{MS}$ & & $13.3^{*}$ & $17.4^{*}$ & 7.8 & 5.3 & 8.8 & 10.3 & 17.1 * \\
\hline 978 & 1-octen-3-ol & $\mathrm{KI}, \mathrm{MS}$ & & 9.8 & $11.8^{*}$ & 0.3 & 5.7 & 3.8 & 9.5 & 3.5 \\
\hline 985 & 3-octanone & $\mathrm{KI}, \mathrm{MS}$ & & 9.3 & 9 & 2.5 & $15.5^{*}$ & 15.2 * & $17.5^{*}$ & $20.8^{*}$ \\
\hline 993 & 3-octanol & $\mathrm{KI}, \mathrm{MS}$ & & 4 & 0 & 0 & 9 & 7.7 & 9 & $15.3^{*}$ \\
\hline 1017 & no ID & & & 0.8 & 0.6 & 1.5 & 7 & 9.7 & $12.5^{*}$ & 10.9 * \\
\hline 1375 & (+)-cycloisosativene & $\mathrm{KI}, \mathrm{MS}$ & + & $11.8^{*}$ & 18 * & 2.8 & 2.5 & $11.8^{*}$ & 12.5 * & $18^{*}$ \\
\hline 1380 & $\alpha$-ylangene & $\mathrm{KI}, \mathrm{MS}$ & + & 13.4 * & $15.5^{*}$ & 0.3 & 0.3 & 7.8 & $12.3^{*}$ & $15.5^{*}$ \\
\hline 1410 & (+)-aromadendrene & $\mathrm{KI}, \mathrm{MS}$ & & 17 * & 8.4 & 0 & 0 & 0 & 0 & 9.6 \\
\hline 1412 & longifolene & $\mathrm{KI}, \mathrm{MS}$ & + & 17.3 * & 11 * & 0 & 0 & 0 & 0 & 10.3 \\
\hline 1418 & SQT unknown & & + & $18^{*}$ & 14 * & 3.9 & 3.3 & 0.1 & 0.4 & 10.3 \\
\hline 1431 & germacrene D & $\mathrm{KI}, \mathrm{MS}$ & + & 12.5 * & 5.8 & 3.7 & 1.5 & 6.7 & 10 & 5.1 \\
\hline 1442 & SQT unknown & & & $17^{*}$ & 10.5 & 0 & 0 & 0 & 0 & 11 * \\
\hline 1445 & SQT unknown & & & $16.5^{*}$ & $11.3^{*}$ & 0 & 0 & 0 & 0 & $10.8^{*}$ \\
\hline 1477 & (E)- $\beta$-farnesene & $\mathrm{KI}, \mathrm{MS}$ & + & $15.5^{*}$ & 7.5 & 9.3 & 0 & 0 & 3.5 & 2.4 \\
\hline 1485 & SQT unknown & & & $16.8^{*}$ & 8.4 & 0 & 0 & 0 & 0 & 9.9 \\
\hline 1488 & SQT unknown & & & $16.5^{*}$ & $13.5^{*}$ & 2.8 & 0 & 0 & 0 & 9.9 \\
\hline 1496 & $\alpha$-muurolene & $\mathrm{KI}, \mathrm{MS}$ & & 13.1 * & 0.3 & 2.9 & 2.9 & 2.9 & 2.9 & 3.1 \\
\hline 1499 & $\beta$-selinene & STD & + & $13.3^{*}$ & $12.5^{*}$ & $17.7^{*}$ & $18.7^{*}$ & 8 & 11.3 & $13^{*}$ \\
\hline 1509 & a-selinene & STD & + & 12.9 * & 7 & 13 * & 14.2 * & 5.2 & 4.5 & 8.5 \\
\hline 1517 & $\beta$-macrocarpene & STD & + & 22.5 * & 19 * & 9.2 & 10.2 & 5.2 & 9.2 & $17.3^{*}$ \\
\hline 1525 & $\beta$-bisabolene & STD & & 19.3 * & 16 * & 10.3 & 10.3 & 2 & 10 & 13.8 * \\
\hline 1533 & trichodiene & $\mathrm{KI}, \mathrm{MS}$ & + & $16.5^{*}$ & $13^{*}$ & 0 & 0 & 0 & 0 & 9 \\
\hline 1547 & SQT unknown & & & 8.3 & 15 * & 4.2 & 0 & 0 & 3.2 & 6.3 \\
\hline 1603 & no ID & & & 1.3 & 10 & $18.7^{*}$ & 11 & 7 & $12.3^{*}$ & 14 * \\
\hline \multicolumn{11}{|c|}{$\begin{array}{l}1 \text { Calulated Kováts' indices (KI calc), based on a homologous series of } n \text {-alkanes } \\
2 \text { Identification by mass spectral libraries (MS), by Kováts' indices (KI) or by comparison with authentic standard (STD) }\end{array}$} \\
\hline \multicolumn{11}{|c|}{3 Volatile markers, confirmed by dynamic open-loop-stripping (OLS) are labelled with ' + ' } \\
\hline \multicolumn{11}{|c|}{$\begin{array}{l}4 \text { Observed differences between 'Fusarium infected' and 'non-infected' following Kruskal-Wallis test; significant differences are } \\
\text { labelled with '*' ( } p<0.01 \text {, critical difference for FG1, FG2 and MIXB is 10.5, for others 11.4), } 3<n<4\end{array}$} \\
\hline \multicolumn{11}{|c|}{$\begin{array}{l}5 \text { Inoculation of maize ears with Fusarium strains, abbreviations } \mathrm{FG} 1, \mathrm{FG} 2=F . \text { graminearum strains, } \mathrm{FSUB}=\text { F.subglutinans } \\
\text { strain, } \mathrm{FV} 1, \mathrm{FV} 2=F \text {. verticillioides strains, } \mathrm{MIXA}=\mathrm{FG} 1 \text { and } \mathrm{FV} 1, \mathrm{MIXB}=\mathrm{FG} 2 \text { and } \mathrm{FV} 2 \text {, source of fungal material is presented } \\
\text { in Tab. 2.1 }\end{array}$} \\
\hline \multicolumn{11}{|c|}{6 Control treatment (ears inoculated with water) } \\
\hline
\end{tabular}

Beside the important group of isoprenoids, other volatiles were identified as markers in hybrid maize trials. The group includes well known compounds that might be emitted by the plant, such as 3-hexen-1-ol and 2-heptanol, but also by the fungus, such as 1-octen-3-ol, 3-octanone and 3-octanol. 


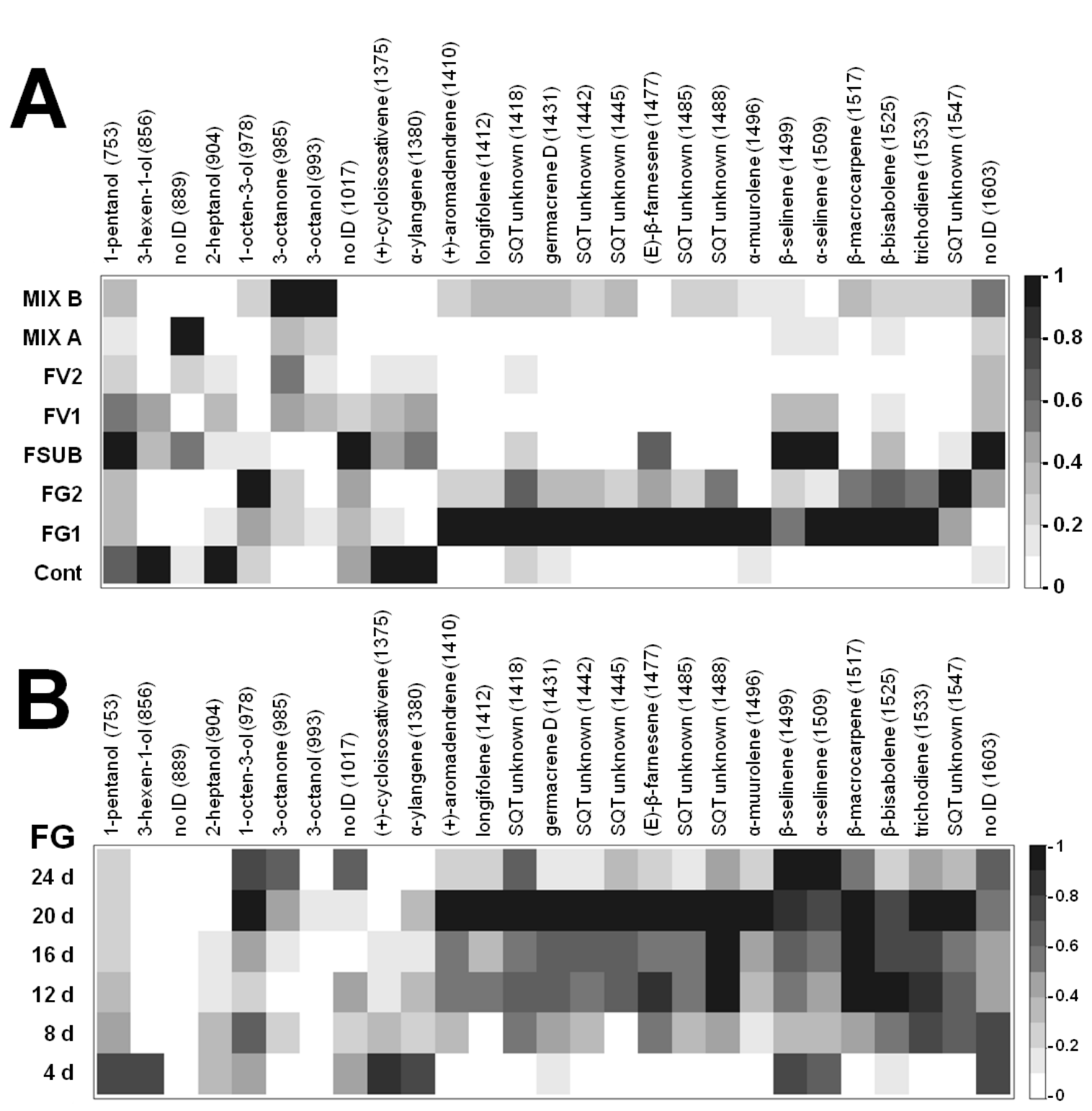

FV



Fig. 2.4 Distribution of selected volatile markers (A) among all Fusarium infection treatments of hybrid maize ears at time point 24 days post-inoculation (dpi) $(3<n<4)$ and (B) in time series among infection with $F$. graminearum (FG, strain FG2) and $F$. verticillioides (FV, strain FV1) at time points $4-24 \mathrm{dpi}(\mathrm{n}=3, \mathrm{~d}=$ days); sampling and analysis by SPME/GC-MS; color coding, as specified in legend, is based on normalized data (first normalization: peak area by total ion chromatogram (TIC), second normalization: means by maximum, $0=$ absent (white), $1=$ maximum (black)), abbreviations for treatments (FG1, FG2, FSUB, FV1, FV2, MIX A, MIX B) are given in Tab. 2.4, Cont = control treatment; markers are ordered by calculated Kováts' retention indices (KI, shown in brackets) 
The dwarf maize variety was selected as reference because it completes its life cycle faster than the commercial hybrid maize (3 months until harvest for dwarf maize, compared to 4 months for hybrid maize) and occupies less space in greenhouse. The selected set of volatile markers from commercial hybrid maize was largely confirmed by the analysis of dwarf maize. Alcohols and carbonyls, such as 3-hexen-1-ol, 2-heptanol and 3-octanone, occurred in the same pattern. Most sesquiterpenes, including the main group of markers, were present. Especially $F$. avenaceum infected dwarf maize ears, showing an intermediate disease severity (89\%) between highly aggressive F. graminearum (100\%) and almost symptomless $F$. verticillioides $(14 \%)$, yielded in a diverse volatile spectra, including main group of VOC markers (except trichodiene) with high signal intensities. However, 8 selected SQT compounds from hybrid maize trials, unknown compound (KI 889), unknown compound (KI 1017), (+)-aromadendrene, unknown SQT (KI 1418), germacrene D, unknown SQT (KI 1442), $\alpha$-muurolene and unknown SQT (KI 1547), were not detectable in dwarf maize.

Fig. 2.6 illustrates symptoms of Fusarium infection on dwarf maize ears.

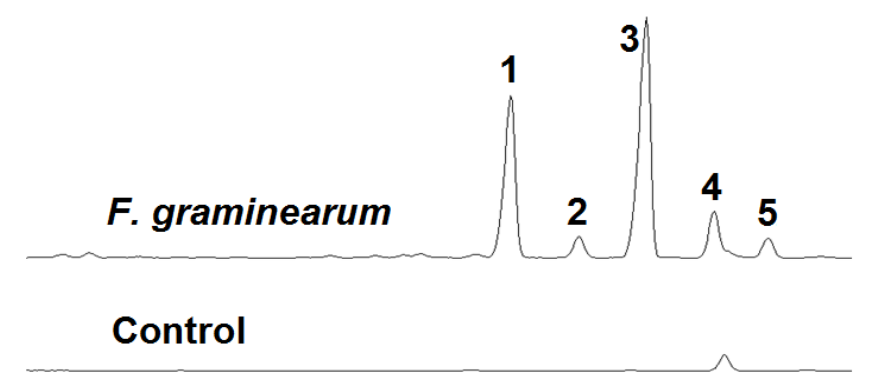

Fig. 2.5 Stacked chromatograms of control and F. graminearum treatment of maize ears, illustrating de novo synthesis of defined main VOC markers for infection, (1) $\beta$-selinene, (2) $\alpha$-selinene, (3) $\beta$-macrocarpene, (4) $\beta$-bisabolene, (5) trichodiene (only in F.graminearum infection)
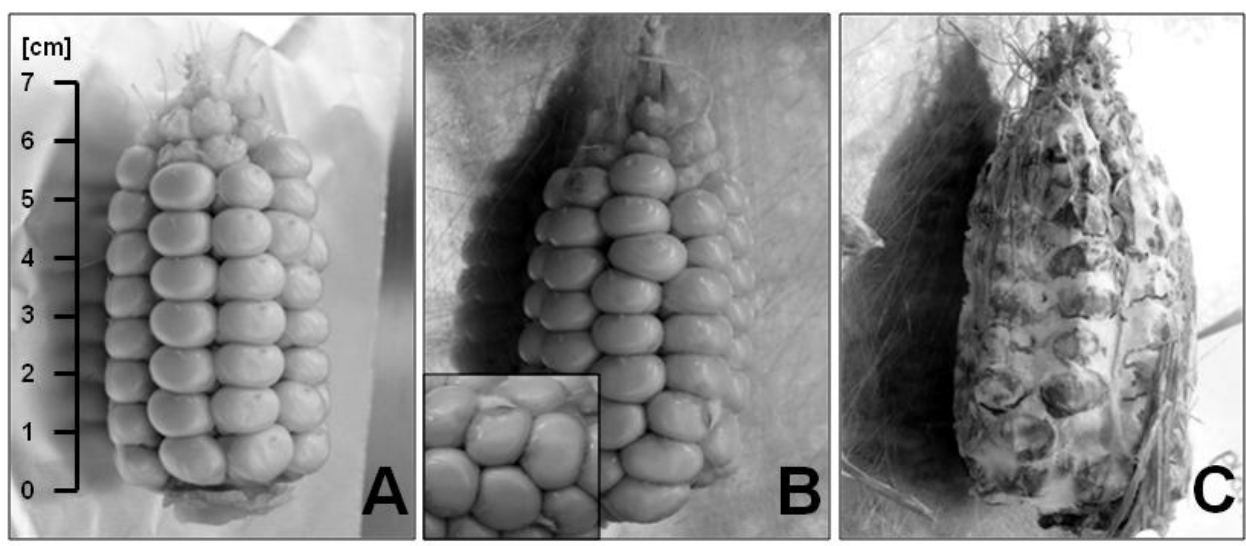

Fig. 2.6 Typical symptoms of Fusarium infection on dwarf maize ears, inoculated with (A) water as control treatment (no symptoms), with (B) F. verticillioides spores and with (C) spores of F.graminearum after 14 days post-inoculation (dpi). F. verticillioides infected ears show fungal mycelium on single kernels, whereas $F$. graminearum infected ears show extensive development of fungal mycelium on the surface 

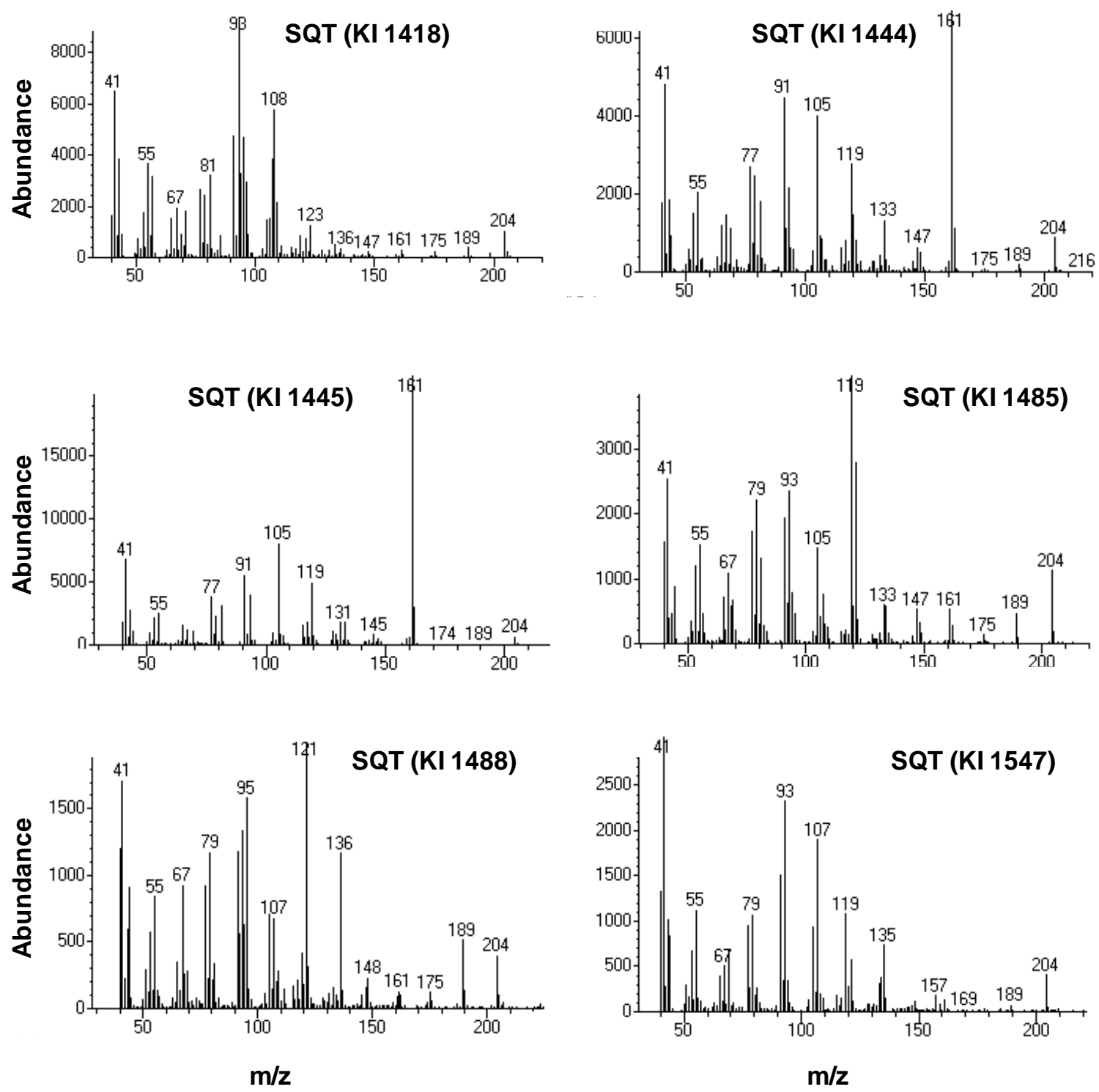

Fig. 2.7 Mass spectra of unknown sesquiterpenoid compounds, defined as markers for ear infection of hybrid maize with $F$. graminearum; $\mathrm{m} / \mathrm{z}=$ mass to charge ratio; sampling and analysis by SPME/GC-MS; mass spectra were extracted from infected maize kernels with F. graminearum strain FG1; calculated Kováts' retention indices (KI) are presented in brackets; SQT = sesquiterpene 


\subsubsection{Volatiles Found in Mixed Infection Treatments of Hybrid Maize by SPME/GC-MS Analysis}

The mixed infection of hybrid maize ears with strains of $F$. graminearum and F. verticillioides (MIX A, MIX B) revealed a mixture of volatile signals that were previously described for both single infections (see also Tab. 2.4 and Fig. 2.4 A). Thus, specific signals that were only detected upon single infection with $F$. graminearum, such as trichodiene or longifolene, were also detected if this species grew together with a competing species. On the other hand, compounds that were most likely released by the fungus (i.e. 3-octanone), which were of minor importance in $F$. graminearum single treatments, showed high abundances if the species grew together with $F$. verticillioides.

\subsubsection{Temporal Release of Volatile Compounds During Pathogenesis (SPME/GC-MS Analysis)}

Temporal emission ( $4-24 \mathrm{dpi}$ ) of the given set of volatile markers is displayed as colour code in Fig. 2.4 B. SPME/GC-MS analysis revealed that the majority of markers for $F$. graminearum (FG2) or $F$. verticillioides (FV1) infection were detectable after 4 to 8 dpi. Most of the markers were continuously released until 24 dpi. Fig. 2.8 illustrates quantitative differences for 6 selected markers in F. graminearum and F. verticillioides infected tissue between 4 dpi and 24 dpi. For the latest time point ( $24 \mathrm{dpi})$ it was demonstrated that 2-heptanol and (+)-cycloisosativene were largely emitted by control plants. In accordance with this, time series revealed a quantitative decrease of both compounds with disease progress of FG2 and FV1. The markers 3-octanone, $\beta$-selinene, $\beta$-macrocarpene and $\beta$-bisabolene showed a temporal increase in abundance. Except for 3-octanone, FG2 induced volatile markers to a larger extent than FV1. For the last time point ( $24 \mathrm{dpi}$ ), it was observed that signal intensities dropped in some cases.

Time point 4 dpi allowed to discriminate between 'healthy' and 'infected'. By time point 8 dpi it was possible to distinguish between red ear rot and pink ear rot. Qualitative biomarkers for $F$. graminearum, identified at $24 \mathrm{dpi}$, never appeared in $F$. verticillioides treatment. Moreover, a quantitative decrease of healthy control signals, such as 3-hexen-1-ol, was observed with progress in maturity. 


\section{2-heptanol}
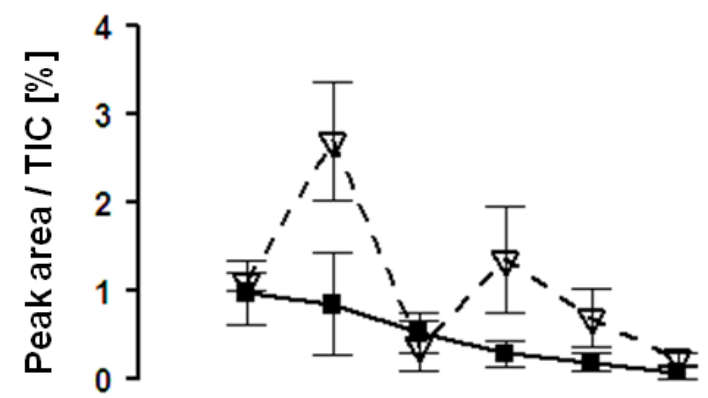

(+)-cycloisosativene
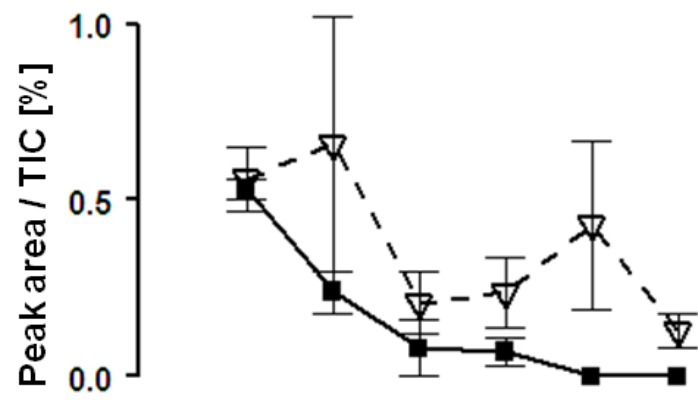

$\beta$-macrocarpene

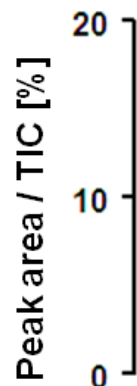

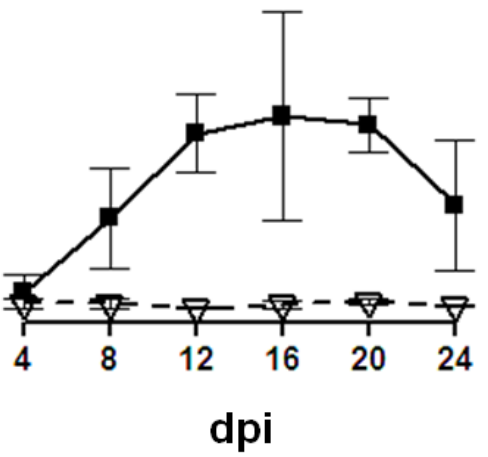

F. graminearum (FG2) 3-octanone
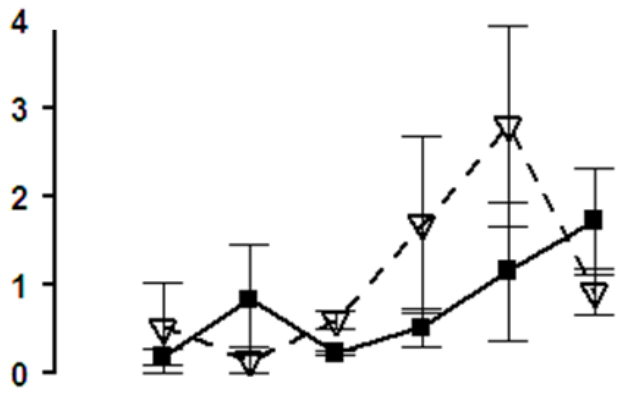

$\beta$-selinene



$\beta$-bisabolene

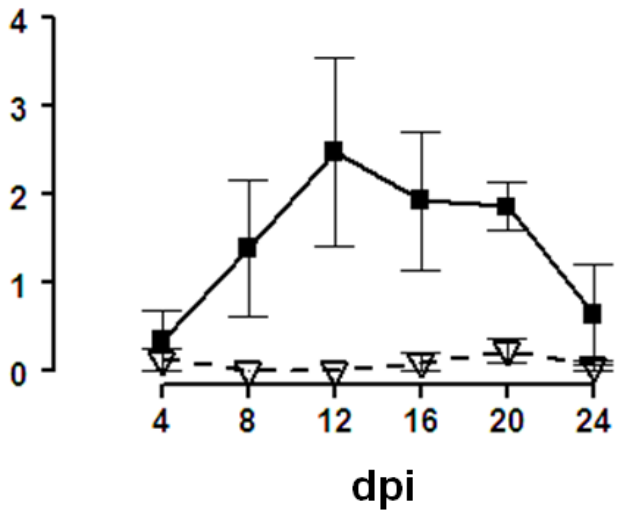

₹ F. verticillioides (FV1)

Fig. 2.8 Effect of Fusarium infection treatment and time point of harvest on the emission of 6 selected volatile markers from hybrid maize kernels; data given as mean peak areas, normalized by total ion chromatogram (TIC) \pm standard error; ears were infected with $F$. graminearum (strain FG2) and F. verticillioides (strain FV1); measurements at 6 time points (4-24 days post-inoculation, dpi); sampling and analysis by SPME/GC-MS; $n=3$ 


\subsubsection{Confirmed VOC Biomarkers by Non-Destructive Sampling (OLS) and Range of Quantities}

Because of the unnatural conditions used for the SPME sampling (i.e. cutting and freezing of sample material), another volatile sampling method was used to confirm that markers identified by SPME/GC-MS were actually emitted from undamaged plants.

In a preliminary test, a non-destructive closed-loop-stripping (CLSA) procedure, which generally has a strong ability to enrich volatiles (Kunert et al., 2009), was tested and turned out to be inadequate for samples with high water content, such as the maturing maize ear. The test revealed that in case of CLSA the accumulation of humidity over a sampling period of several hours was very high, which resulted in a soaking of the charcoal matrix and subsequently the blocking of volatiles adsorption. The reduction of water content in CLSA was tested with $100 \mathrm{mg}$ magnesium sulphate (powder, 99.5\%, Sigma-Aldrich, Taufkirchen, Germany), packed in glass cartridges between glass wool. The $\mathrm{MgSO}_{4}$ cartridge was placed between sample and adsorbent. It became soaked with water within a few hours, which consequently clogged the complete system. Because of the impropriety of the closed-loop procedure, a dynamic OLS, with continuous supply of ambient air and equal temperature and humidity conditions, compared to greenhouse conditions, was applied (conditions were assessed, data not shown).

The $24 \mathrm{~h}$ OLS system on hybrid maize plants in greenhouse confirmed 10 volatile markers of the previously described marker set with the static sampling (37 \%, see also Tab. 2.4). Confirmed markers included the sesquiterpene cluster identified by SPME/GC-MS, with the exception of $\beta$-bisabolene, (+)-cycloisosativene, $\alpha$-ylangene, longifolene, an unknown SQT (KI 1418), germacrene D and (E)- $\beta$-farnesene. Quantitative results, as mean peak area, normalized by peak area of nonyl acetate as internal standard, are presented in Tab. 2.5.

Estimating the concentration of sesquiterpenoid volatiles was performed on a flame ionization detector (FID) and revealed that SQTs were emitted from infected maize ears at $0.03-0.3 \mu \mathrm{g}$ $24 \mathrm{~h}^{-1}$ OLS sampling. 
Tab. 2.5 Occurrence of volatile markers in dynamic sampling (OLS) on Fusarium infected and non-infected maize ear material at time point 24 days post-inoculation (dpi) (infection treatments are explained in Tab. 2.4)

\begin{tabular}{|c|c|c|c|c|c|c|c|c|}
\hline \multirow{2}{*}{$\begin{array}{l}\text { VOC } \\
\text { No. }{ }^{1}\end{array}$} & \multicolumn{8}{|c|}{ Mean peak area normalized by peak area of internal standard ${ }^{2} \pm$ standard error } \\
\hline & CONT & FG1 & FG2 & FSUB & FV1 & FV2 & MIXA & MIXB \\
\hline 1 & $0.33 \pm 0.05$ & $0.22 \pm 0.08$ & $0.47 \pm 0.09$ & $0.76 \pm 0.16$ & $0.93 \pm 0.26$ & $0.97 \pm 0.22$ & $0.87 \pm 0.21$ & $0.98 \pm 0.06$ \\
\hline 2 & $0.18 \pm 0.02$ & $0.16 \pm 0.07$ & $0.32 \pm 0.05$ & $0.50 \pm 0.12$ & $0.63 \pm 0.18$ & $0.66 \pm 0.14$ & $0.60 \pm 0.20$ & $0.73 \pm 0.05$ \\
\hline 3 & $0.14 \pm 0.02$ & $0.32 \pm 0.21$ & $0.21 \pm 0.06$ & $0.25 \pm 0.03$ & $0.12 \pm 0.00$ & - & $0.26 \pm 0.16$ & $0.37 \pm 0.08$ \\
\hline 4 & $0.34 \pm 0.08$ & $0.45 \pm 0.06$ & $0.41 \pm 0.11$ & $0.62 \pm 0.04$ & $0.40 \pm 0.02$ & $0.43 \pm 0.02$ & $0.32 \pm 0.07$ & $0.42 \pm 0.01$ \\
\hline 5 & - & $0.17 \pm 0.11$ & $0.57 \pm 0.16$ & $0.35 \pm 0.08$ & $0.24 \pm 0.08$ & $0.27 \pm 0.08$ & $0.45 \pm 0.18$ & $0.64 \pm 0.02$ \\
\hline 6 & - & $0.06 \pm 0.05$ & $0.17 \pm 0.01$ & $0.11 \pm 0.06$ & $0.05 \pm 0.05$ & - & $0.10 \pm 0.10$ & $0.18 \pm 0.02$ \\
\hline 7 & - & $0.46 \pm 0.44$ & $1.36 \pm 0.47$ & $0.23 \pm 0.04$ & $0.22 \pm 0.06$ & $0.24 \pm 0.05$ & $1.44 \pm 0.73$ & $0.98 \pm 0.36$ \\
\hline 8 & - & $0.09 \pm 0.08$ & $0.21 \pm 0.05$ & $0.04 \pm 0.04$ & $0.11 \pm 0.06$ & $0.10 \pm 0.05$ & $0.30 \pm 0.21$ & $0.23 \pm 0.06$ \\
\hline 9 & - & $0.70 \pm 0.67$ & $1.56 \pm 0.24$ & $0.05 \pm 0.05$ & $1.59 \pm 1.59$ & $0.04 \pm 0.04$ & $0.97 \pm 0.53$ & $1.29 \pm 0.36$ \\
\hline 10 & - & $0.09 \pm 0.08$ & $0.18 \pm 0.04$ & - & - & - & $0.06 \pm 0.06$ & $0.05 \pm 0.05$ \\
\hline 2 & $\begin{array}{l}\text { (1) (+)-cycloi } \\
\text { (6) (E)- } \beta \text {-farne } \\
\text { Nonyl acetate }\end{array}$ & $\begin{array}{l}\text { sativene (2 } \\
\text { ene (7) } \beta \text {-s€ } \\
\text { s internal s }\end{array}$ & $\begin{array}{l}\text {-ylangene }(3 \\
\text { ene (8) } \alpha \text {-se } \\
\text { idard, } 8 \mu \mathrm{g} \mathrm{m}\end{array}$ & ngifolene (4 & $\overline{Q T}(\mathrm{KI} 141$ & $\begin{array}{l}\text { (5) germacrer } \\
\text { ) trichodiene }\end{array}$ & & \\
\hline
\end{tabular}

\subsubsection{Systemic Distribution of VOCs}

Since spore suspensions were inserted into the silk channel of the maize ear, the infection started at the tip of the ear and moved downwards. Fungal biomass, measured with quantitative real-time PCR, was highest in the upper part of the ear for both species, $F$. graminearum and $F$. verticillioides (data not shown). As expected, signal intensities of volatile markers were highest in the upper part of the maize ear and decreased longitudinally moving towards the base of the ears (data not shown). Furthermore, none of the selected volatile markers from infected ears could be detected in collected maize leaves.

\subsubsection{Volatile Spectra of in vitro Cultures on Maize and Rice and Effect of Infected Maize Volatiles on Fungal Growth}

It is known that the volatile profile changes according to the substrate (i.e. plant material, artificial medium). To check whether the VOC spectrum changes on dead (sterile) plant material, the emission of volatile signals was investigated under in vitro conditions. Volatile markers from the Fusarium infected maize ears in greenhouse (identified with SPME/GC-MS) were not detected under in vitro conditions on autoclaved maize material. 
Under in vitro conditions on sterile maize kernels, few terpenoid compounds that did not appear in greenhouse trials were detected (no data presented). In vitro cultures on autoclaved rice kernels did not release volatiles of the selected marker set for hybrid maize as well. Similar to sterile maize, few terpenoid compounds were detected (data not shown).

The effect of VOC extracts, derived from the maize-pathogen system, on the growth of Fusarium spp. was observed under in vitro conditions (maintainable volume of DCM was determined empirically in a preliminary experiment, data not shown). DCM extracts of Fusarium infected maize ear material had no significant effect on the fungal growth of $F$. graminearum and $F$. verticillioides.

\subsubsection{Fungal Biomass, Disease Severities and Mycotoxin Production in Infected Hybrid Maize}

Concentration of fungal DNA, as indicator for fungal biomass in infected plant tissue, was investigated. Quantitative real-time PCR revealed a proper infection for all treatments with Fusarium strains at time point 24 dpi (few replicates, showing an inadequate infection were excluded from analysis). In single infection treatments with $F$. graminearum, the biomass of FG1 was higher than FG2, whereas the F. verticillioides strain FV1 produced a higher biomass than FV2 (data not shown). In time series, the presence of fungal DNA of $F$. graminearum and F. verticillioides was confirmed for all 6 time points from 4 to 24 dpi. In the mixed infection treatments at $24 \mathrm{dpi}$, the quantitative real-time PCR indicated a complete suppression of FG1, if the strain grew together with FV1. Fungal biomass of FG2 was not suppressed by mixed infection. No suppression of $F$. verticillioides biomass (both strains) was observed in the mixed infection treatments.

Mean disease severities at time point 24 dpi were estimated to be $91 \%$ for FG1, $50 \%$ for FG2, $60 \%$ for FV1, $7 \%$ for FV2, $25 \%$ for FSUB, $13 \%$ for MIX A and $75 \%$ for MIX B. Disease progress, monitored as disease severity at each time point of the time series, was continuous from 4 to 24 dpi for both species.

The relationship between formation of infection specific VOCs and toxic secondary metabolites of the fungus was investigated. An overview of mycotoxin concentrations after 24 dpi is given in Tab. 2.6. In single infection treatments, FG1 produced NIV and, respectively, FG2 produced DON to a high extent (DON and NIV concentration $>50 \mathrm{mg} \mathrm{kg}^{-1}$ on average). Estrogenic ZEN was irregularly produced by both F. graminearum strains. 
Fumonisins B1 and B2 were produced by both F. verticillioides strains, whereas FV1 produced fumonisins on a larger scale.

Tab. 2.6 Influence of Fusarium infection treatment on the formation of mycotoxins deoxynivalenol (DON), zearalenone (ZEN), nivalenol (NIV) and fumonisins B1 and B2 (FB1, FB2) in infected hybrid maize ears after 24 days past inoculation (dpi)

\begin{tabular}{|c|c|c|c|c|c|}
\hline \multirow{2}{*}{ Infection treatment ${ }^{a}$} & \multicolumn{5}{|c|}{ Toxin concentration [mg kg ${ }^{-1}$ dry weight \pm standard error ] ${ }^{b}$} \\
\hline & DON & ZEN & NIV & FB1 & FB2 \\
\hline CONT & - & - & - & - & - \\
\hline FG1 & - & - & $>50^{c}$ & - & - \\
\hline FG2 & $>50^{c}$ & $0.55 \pm 0.45$ & - & - & - \\
\hline FV1 & - & - & - & $>10^{c}$ & $>10^{c}$ \\
\hline FV2 & - & - & - & $1.07 \pm 0.45$ & $0.51 \pm 0.22$ \\
\hline FSUB & - & - & - & - & - \\
\hline $\operatorname{MIX} A(F G 1, F V 1)$ & - & - & - & $1.67 \pm 1.33$ & $0.23 \pm 0.14$ \\
\hline MIX B (FG2, FV2) & $>50^{c}$ & $0.03 \pm 0.03$ & - & $0.12 \pm 0.12$ & $0.08 \pm 0.08$ \\
\hline Analysis with HPLC-MS, $4<\mathrm{n}<5$ & \multicolumn{5}{|c|}{ CONT = control treatment (water inoculated), fungal treatments are explained in Tab. 2.4} \\
\hline \multicolumn{6}{|c|}{$\begin{array}{l}\text { c Concentration exeeds range of detection (trichothecenes (DON, NIV): }>50 \mathrm{mg} \mathrm{kg}^{-1} \text {; fumonisins (FB1, } \\
\text { FB2): }>10 \mathrm{mg} \mathrm{kg}^{-1} \text { ) }\end{array}$} \\
\hline
\end{tabular}

In time series, with $F$. graminearum strain $\mathrm{FG} 2$ and $F$. verticillioides strain FV1, DON was detected at $4 \mathrm{dpi}$. As expected, fumonisins were detected from a later time point of $16 \mathrm{dpi}$. Results revealed no clear trend of time-dependent DON and fumonisin accumulation in maize ear (see Fig. 2.9).

Relation between emission of volatile markers and mycotoxin concentration over time is presented in Fig. 2.9. It is illustrated that the relative quantity of trichodiene, as volatile precursor in biosynthesis of trichothecenes, showed roughly the same pattern as DON production over all time points. The correlation between quantity of trichodiene and DON was high until $20 \mathrm{dpi}\left(\mathrm{R}^{2}\right.$ 20dpi $\left.=0.86\right)$, but dropped afterwards. 3-Octanone, a common volatile compound with probable fungal origin and highest abundances in F. verticillioides treated maize ears, was found to show same temporal development than FB1 concentration. Hence, mean quantities of 3-octanone and FB1 from $F$. verticillioides infected maize ears correlated with $\mathrm{R}^{2}=0.86$ over time. 

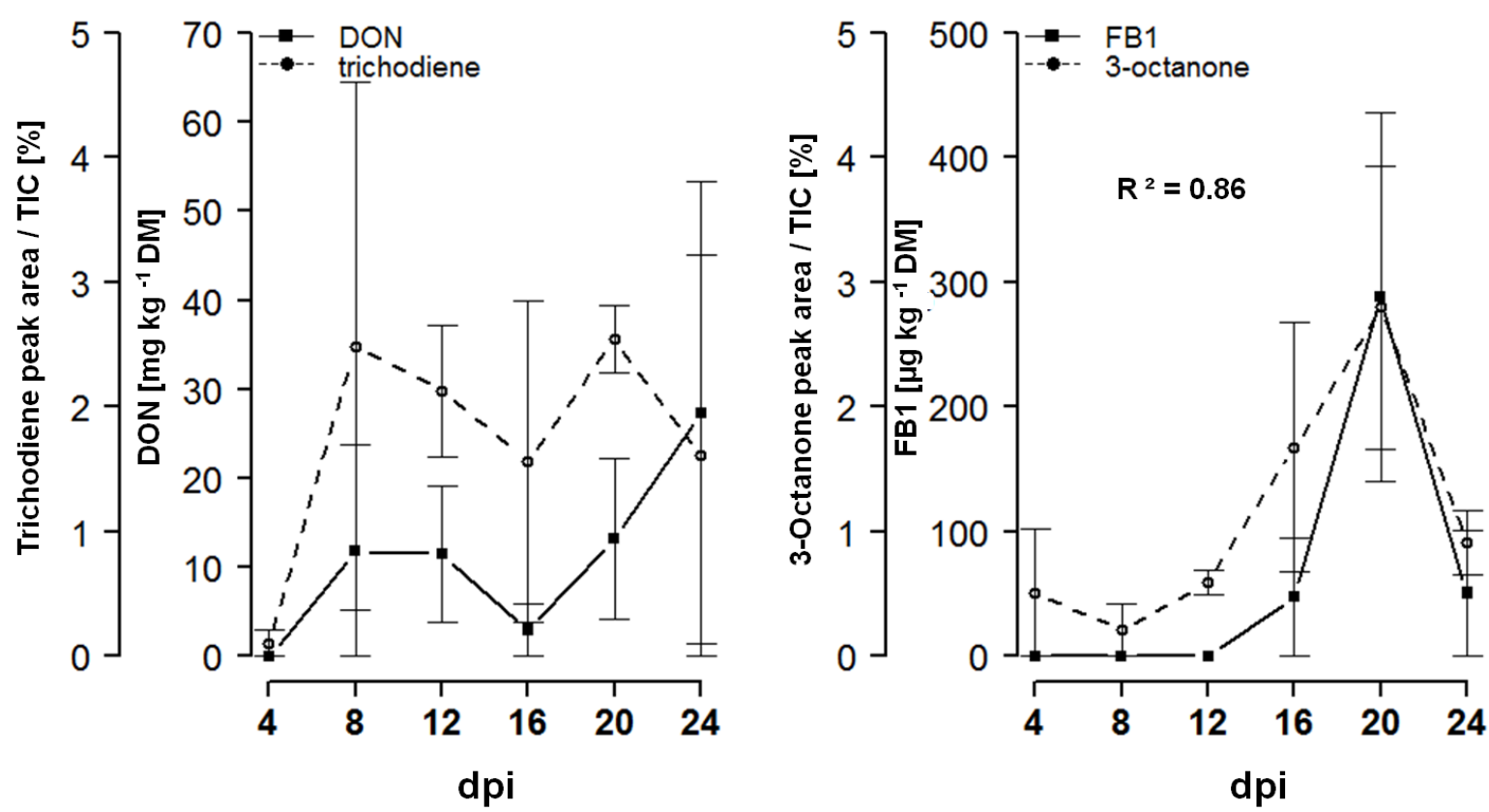

Fig. 2.9 Relative quantity of volatile compounds trichodiene and 3-octanone as mean peak area, normalized by total ion chromatogram (TIC) \pm standard error, and mycotoxin concentration (deoxynivalenol (DON, $\mathrm{mg} \mathrm{kg}^{-1}$ dry matter (DM) \pm standard error), fumonisin B1 (FB1, $\mathrm{g} \mathrm{kg} \mathrm{k}^{-1} \mathrm{DM} \pm$ standard error)) in hybrid maize ears over 6 time points from 4 to 24 days post-inoculation (dpi); toxin analysis was performed by HPLC-MS, VOC analysis was conducted by SPME/GC-MS, $n=3$

In mixed infection treatments with $F$. graminearum and $F$. verticillioides, strain FG1 stopped the production of NIV (in accordance with biomass), whereas DON production by FG2 was not affected. The concentrations of Fumonisins FB1 and FB2, produced by $F$. verticillioides strains, were lower in the mixed infection treatments compared to single infection.

\subsubsection{Transcription of Terpene Synthase Genes in Infected Dwarf Maize}

Transcript levels of terpene synthase genes tps6 and tps11, involved in the formation of $\beta$-bisabolene and $\beta$-macrocarpene (Koellner et al., 2008), were investigated. The transcription of tps6 and tps11 was not found to be significantly upregulated by infection of dwarf maize ears with Fusarium species (Scheffé test, p < 0.05). Nevertheless, the transcription level of tps6/11, expressed as relative fold change compared to the housekeeping gene, was $36 \mathrm{x}$ higher than control in the $F$. graminearum treatment and $24 \mathrm{x}$ higher than control in the F. avenaceum treatment. Transcription level in F. verticillioides was only $4 \mathrm{x}$ higher than in the non-infected control. We found no correlation between $\beta$-bisabolene and $\beta$-macrocarpene formation and transcription of tps6/11 (data not shown). Relative fold changes in transcription of terpene synthase genes, and correlation between disease severity and transcription level are illustrated in Fig. 2.10 A and B. 

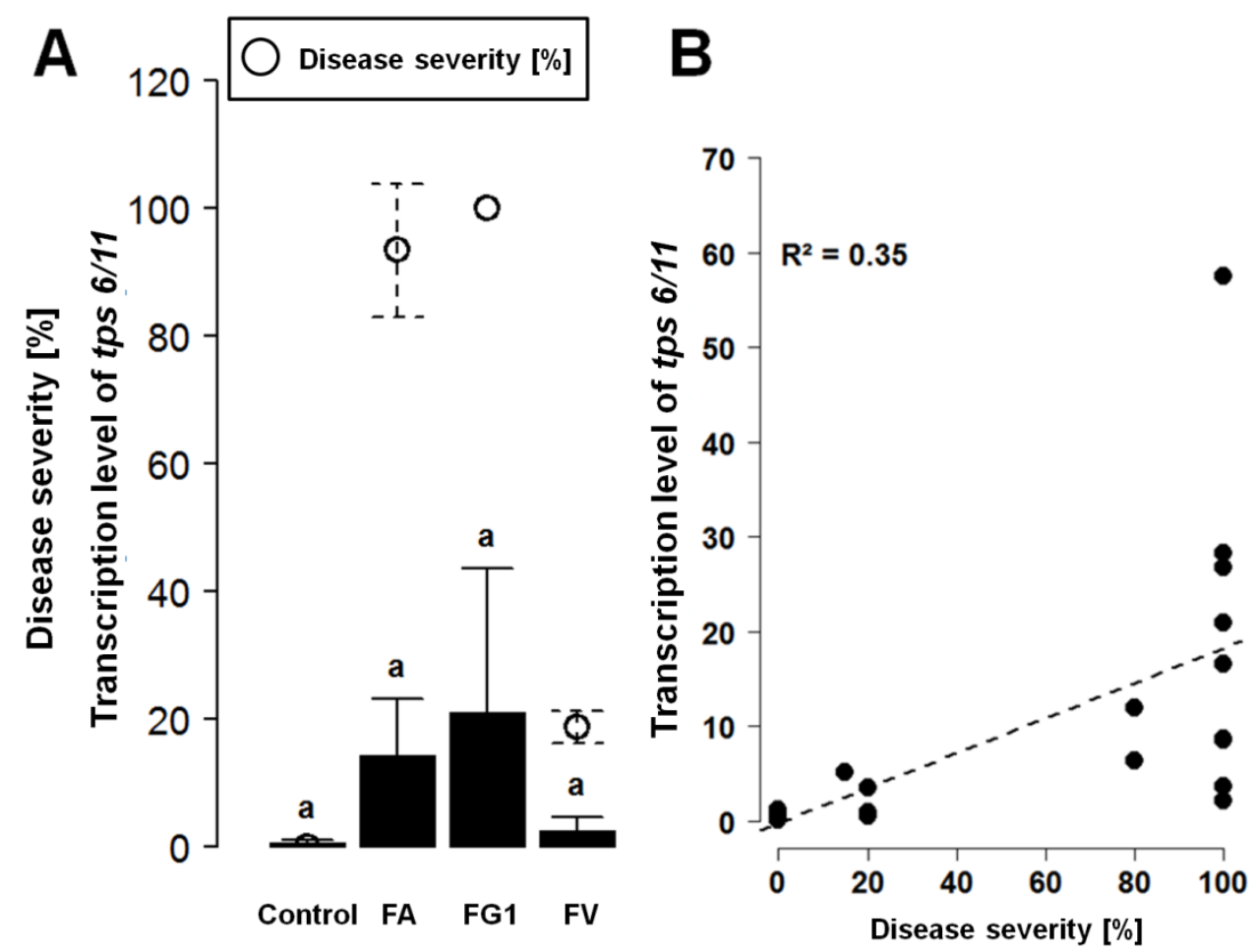

Fig. 2.10 Effect of fungal infection on the transcription of terpene synthase genes involved in plant defense reactions: (A) Influence of Fusarium infection treatment on transcription level of terpene synthase genes tps 6 and tps 11 (expressed as relative fold increase, compared to transcription of house-keeping gene) and estimates of infection as percentage of disease severity, no significant differences in tps6/11 transcription between treatments were found (Scheffé test); control = water inoculated ears, fungal treatments are described in Tab. 2.4; $4<\mathrm{n}<6$; (B) Low correlation between disease severity and transcription of terpene synthase genes $\left(R^{2}=0.35\right)$

\subsubsection{Oxylipins in Fusarium Infected Hybrid Maize Ears}

Results of oxylipin analysis in infected hybrid maize ears illustrate clear differences between control treatment and Fusarium infected material. Levels of oxidized fatty acids (by 9- and 13-lipoxygenases) were largely upregulated in infected tissue. Fig. 2.11 presents concentrations of oxidized fatty acids (9/13-hydroxyoctadecadienoic acid (9/13-HOD) and 9-/13-hydroxyoctadecatrienoic acid (9/13-HOT)) among infection treatments. F. graminearum strain FG1 induced highest levels of oxylipins, whereas infections with both F. verticillioides strains did not result in statistically significant increases compared to the control treatment. Maize samples, treated with mixed spore suspensions of $F$. graminearum and $F$. verticillioides, expressed intermediate levels of plant oxylipins. No significant differences between fatty acids, such as $\alpha$-linoleic acid (18:3) and linoleic acid (18:2), were observed among infection treatments (data not shown). Thus, there was no effect of the infection treatment on the availability of substrates for lipid peroxidation. 

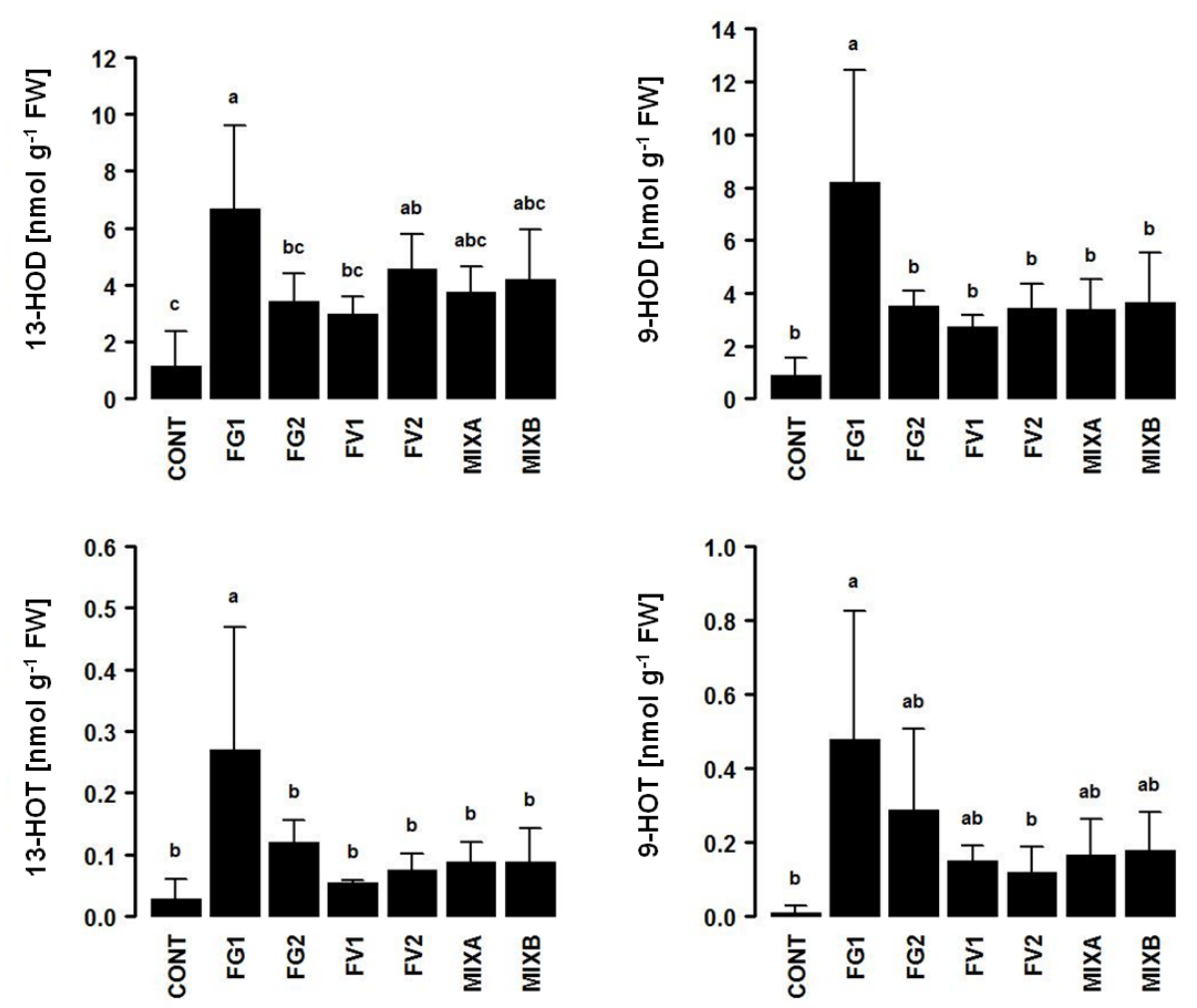

Fig. 2.11 Influence of Fusarium infection treatment on the formation of oxidized fatty acids (nmol g ${ }^{-1}$ fresh weight (FW); analysis of maize samples was performed with GC-MS; within plots different letters (a-c) represent statistical differences between treatments (Scheffé test $(p<0.05), 4<n<7)$. Abbreviations: CONT = water inoculated ears, Fusarium treatments FG1, FG2, FV1, FV2, MIX A and MIX B are explained in Tab. 2.4, 13-HOD = 13-hydroxyocatdecadienoic acid, 9-HOD = 9-hydroxyocatdecadienoic acid, 13-HOT = 13-hydroxyoctadecatrienoic acid, 9-HOD = 9-hydroxyoctadecatrienoic acid

\subsubsection{Induction of Volatiles Upon Stem Treatment of Dwarf Maize with Jasmonic Acid and Ethephon}

The stem elicitation assay in dwarf maize plants revealed few differences between control and phytohormone treatments. Abundances of sesquiterpenoids (+)-cycloisosativene and $\alpha$-ylangene were slightly upregulated by ethephon and JA-ethephon treatments compared to the control treatment of collected stem sections. Abundances in volatile $\beta$-caryophyllene, which is ubiquitous and was not identified as volatile marker, were insignificantly upregulated by all phytohormone treatments. Same trends were observed under in vivo conditions (data not shown). 


\subsubsection{Zealexins and Phytohormones in Fusarium Infected Dwarf Maize Ears}

Zealexins and phytohormones play a role in plant defense reactions. Changes in the formation of these secondary metabolites upon infection with Fusarium spp. were checked. The sesquiterpenoid zealexins A1 and A3 were absent in the water inoculated control and present in Fusarium infected dwarf maize material. The detected mass spectra of zealexins and the co-occurrence of zealexins and their known volatile precursors, $\beta$-bisabolene and $\beta$-macrocarpene, are presented in Fig. 2.13. A strong correlation between quantities of zealexin A3 and its precursor zealexin A1 $\left(\mathrm{R}^{2}=0.899\right)$ was found.

No statistical difference was found between concentrations of JA among Fusarium infection treatments (see Fig. 2.12) of dwarf maize ears.

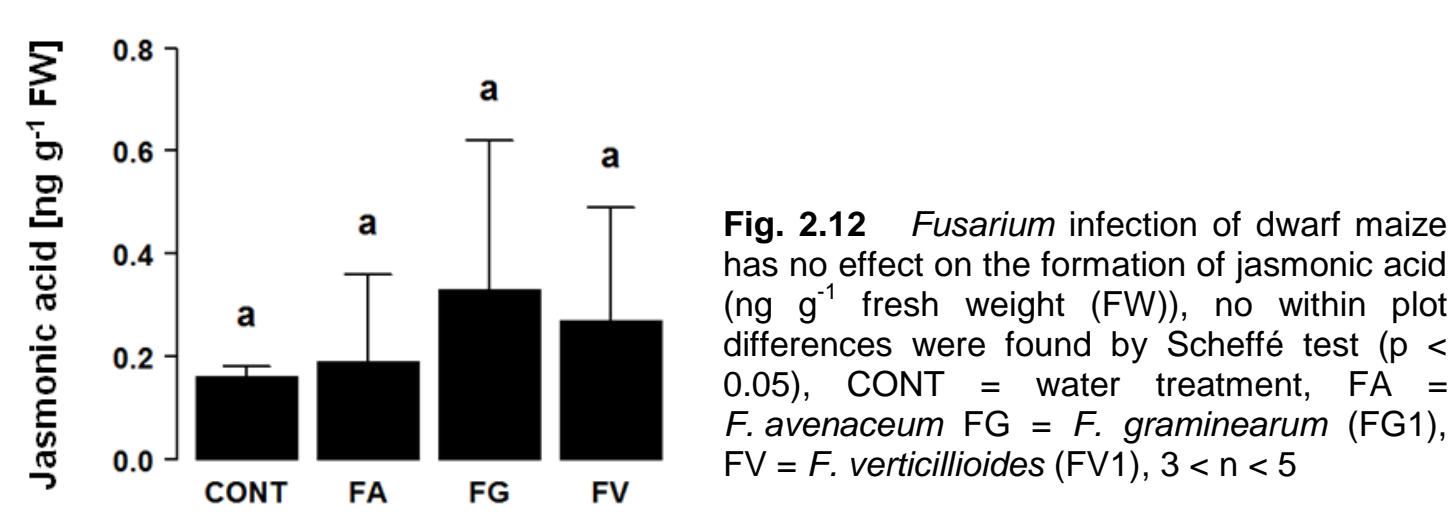




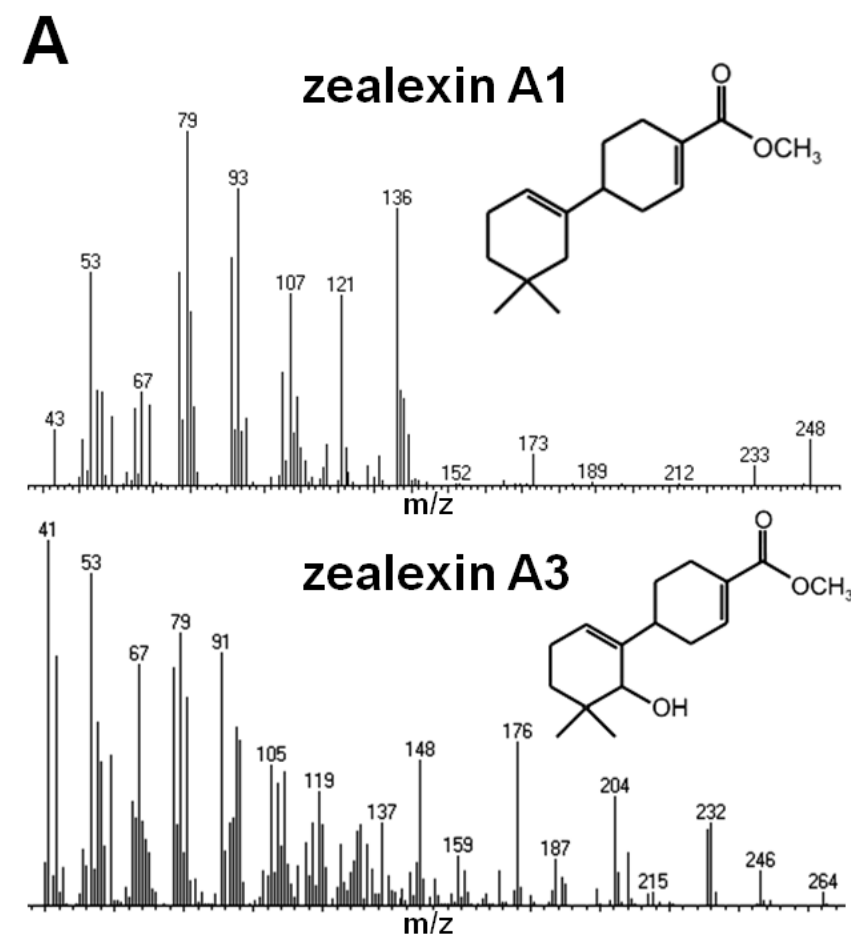

B

(2E,6E)-farnesyl diphosphate

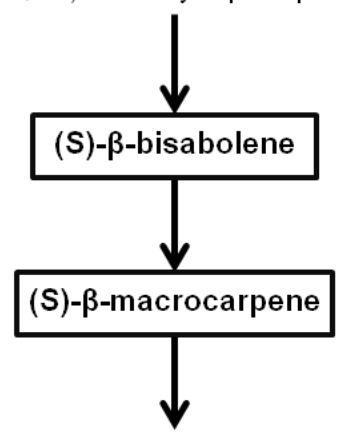

[4-(5,5-dimethylcyclohex-1-en-1-yl)cyclohex-1-en-1-yl] methanol

4-(5,5-dimethylcyclohex-1-en-1-yl)cyclohex-1-en-1- carbaldehyde
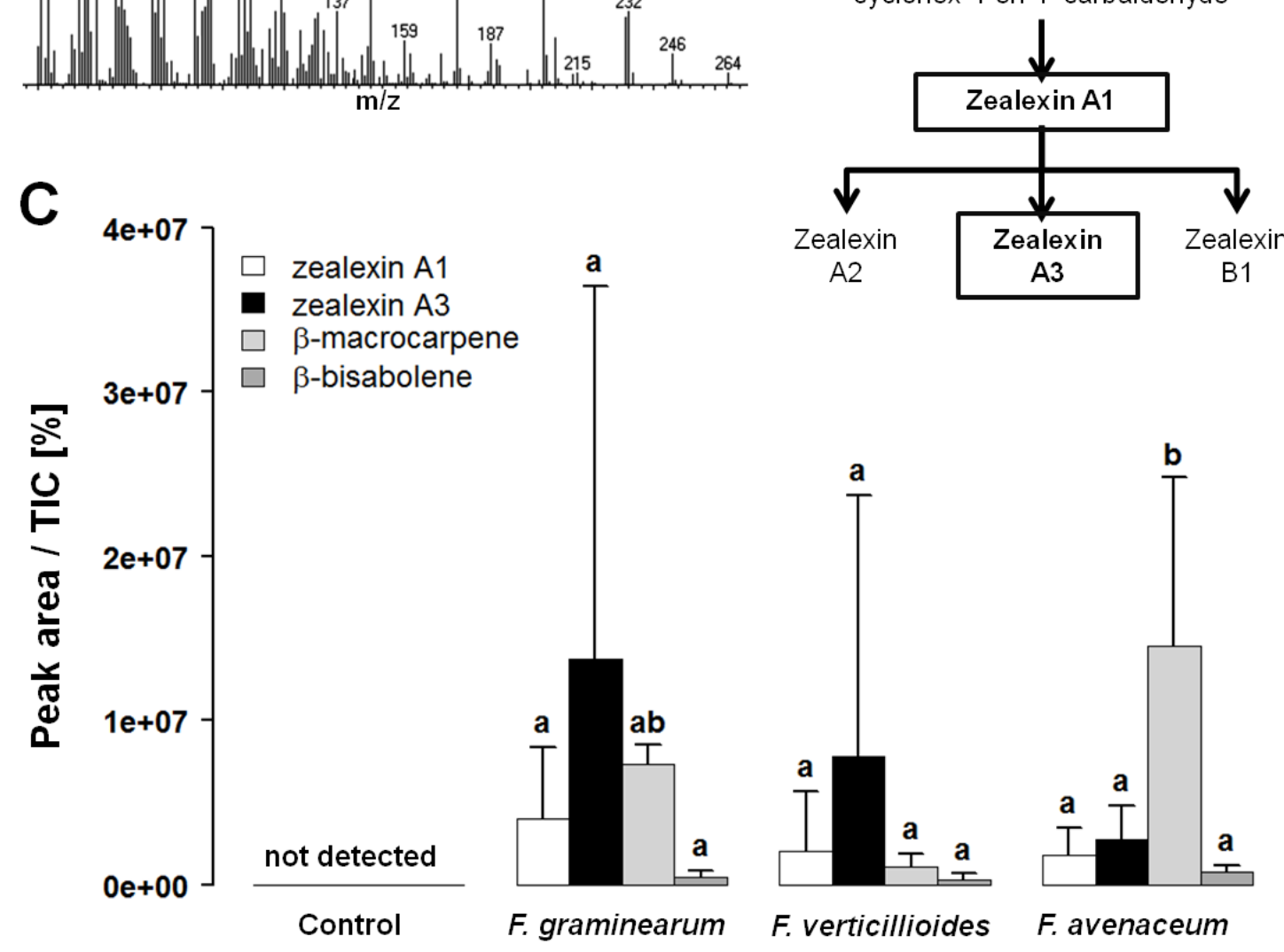

Fig. 2.13 Occurrence of zealexins in dwarf maize ears infected with Fusarium spp. (analysis by GC-MS after derivatization), (A) mass spectra of zealexin A1 / A3, structures were published by Huffaker et al. (2011), $\mathrm{m} / \mathrm{z}$ = mass-to-charge-ratio, (B) zealexin biosynthesis includes precursors $\beta$-bisabolene and $\beta$-macrocarpene (pathway modified from http://pmn.plantcyc.org/PLANT/NEWIMAGE?type=PATHWAY\&object=PWY-6888; accessed November 03, 2012), (C) influence of infection treatment on the formation of zealexins and their volatile precursors $\beta$-macrocarpene and $\beta$-bisabolene in dwarf maize $(3<n<6)$, given as peak area, normalized by total ion chromatogram (TIC) \pm standard deviation, treatments: Control = water treatment, F. graminearum (strain FG1), $F$. verticillioides (strain $\mathrm{FV} 1$ ), $F$. avenaceum (strain $\mathrm{FA}$ ), statistical differences between treatments within terpenoid compounds are illustrated with within plots different letters (Scheffé test, $p<0.05$ ) 


\subsection{Discussion}

In this study, we identified a set of 27 volatile markers (see Tab. 2.4 and Fig. 2.4) specific to maize ears infected with toxigenic Fusarium spp., such as F. graminearum and $F$. verticillioides, the causal agents of ear rot in maize and producers of toxic metabolites. These volatile markers included compounds already reported in fungi and plants. For example 3-octanone, a common eight carbon containing $\left(\mathrm{C}_{8}\right)$ volatile of probable fungal origin, which has been suggested to be a marker of microbial spoilage (Magan \& Evans, 2000; Jeleń \& Wasowicz, 1998; Boerjesson et al., 1989; Kaminski et al., 1974). Six carbon containing volatiles $\left(\mathrm{C}_{6}\right)$, such as $3-(\mathrm{Z})$-hexenal, were similarly reported to be induced in plants upon pathogen or herbivore attack and some studies described their induction after abiotic stress (Holopainen \& Gershenzon, 2010; Matsui, 2006; Rostás et al., 2006; Engelberth et al., 2004; Farag et al., 2004).

In contrast to these $\mathrm{C}_{6}$ and $\mathrm{C}_{8}$ hydrocarbons, which are widespread among plants and microbes, we also identified a group of sesquiterpenoid markers for Fusarium infection of maize ears, which occurred only in our pathosystem and a limited number of other plant species. These sesquiterpenes included five compounds, which appeared as major signals in the chromatograms of infected maize ears (see Fig. 2.5). Among these five major compounds, two ( $\beta$-macrocarpene and $\beta$-bisabolene) are supposed to be precursors of zealexins, a class of phytoalexins. Huffaker et al. (2011) reported these acidic non-volatile sesquiterpenes to occur in young maize stems upon fungal infection, herbivory and treatments with stress hormones. Beside zealexins, the group also detected $\beta$-macrocarpene in fungal infected maize stems. Previously, Koellner et al. (2008) investigated volatiles from maize leaves after herbivory and could not detect $\beta$-macrocarpene but low amounts of $\beta$-bisabolene. In the present study we demonstrated the occurrence of $\beta$-macrocarpene and $\beta$-bisabolene as well as the induction of zealexins A1 and A3 in Fusarium infected maize ears. These results demonstrate that in addition to non-volatile zealexins, maize ears also produce large amounts of sesquiterpenoid volatiles, which might possibly be involved in direct defense against pathogens, but could also play a role in plant-plant signalling.

Major sesquiterpene infection markers further included $\alpha$ - and $\beta$-selinene, known components of celery oil (Rao et al., 2000). In plants, different from maize, both compounds have been reported to be involved in plant defense against pathogens and insects (Tuetun et al., 2008; Momin et al., 2000) and might consequently play a similar role in maize. 
The last major terpenoid infection marker was trichodiene, the well known volatile precursor in the biosynthesis of trichothecenes (Jeleń et al., 1997). The compound was exclusively induced upon $F$. graminearum infection. Trichodiene was extensively described as a volatile marker for $F$. graminearum, F. culmorum and the associated mycotoxin contamination (Girotti et al., 2012; Perkowski et al., 2008).

Other sesquiterpenoid markers for Fusarium infection (minor signals in chromatograms from infected maize, compared to major group of markers) included (+)-aromadendrene, (E)- $\beta$-farnesene and $\alpha$-muurolene, which have already been described in infected plants by others (Sabater-Jara et al., 2010; Soković et al., 2009; Schnee et al., 2006; Bernasconi et al., 1998) and a set of unidentified sesquiterpenoid compounds. In summary, among the 27 volatile markers for Fusarium infection identified in this study, sesquiterpenes were the most infection specific.

The source of volatile markers (maize plant or fungus), identified in the present study is not always evident. $\mathrm{C}_{6}$ and $\mathrm{C}_{8}$ hydrocarbons or sesquiterpenes can be produced by numerous plants and microbes, and possibly by the intimate interaction of both classes. Monitoring the emission of volatiles over time and linking the volatile levels with factors, such as fungal biomass, mycotoxin analysis or plant gene expression, can help to distinguish which organism (plant or fungus) is behind the production of volatiles. For example, we found a strong correlation between normalized peak area of $\mathrm{C}_{8}$ volatile 3-octanone and $\mathrm{FB} 1$ concentration in F. verticillioides infected maize ears over time $\left(\mathrm{R}^{2}=0.86\right.$, Fig. 2.9), which supports the assumption that the volatile compound is of fungal origin. We further observed the cooccurrence of volatile trichodiene and DON (Fig. 2.9) in F. graminearum infected samples until $20 \mathrm{dpi}\left(\mathrm{R}^{2}{ }_{20 \mathrm{dpi}}=0.86\right)$. After this time stage, the volatile precursor dropped quantitatively, while the toxin concentration still increased. One reason might be that quantitative fluctuations between precursor and product of fungal origin undergo a temporal shift. Although the differences were not significant, we observed high fold changes in the transcription of terpene synthase genes tps6/11 in infected dwarf maize ears compared to control (Fig. 2.10). These plant genes encode terpene synthases that catalyse the formation of $\beta$-macrocarpene from $\beta$-bisabolene intermediates (Koellner et al., 2008), which indicates that both detected volatiles are of plant origin.

Interestingly, we could not detect any of the 27 selected volatile markers for Fusarium infection of maize ears in the headspace of in vitro cultures with Fusarium spp. growing on 
sterilized maize and rice kernels. Therewith, our results are in contrast to other studies reporting the occurrence of trichodiene, and also other common terpenes, under these conditions (Girotti et al., 2010; Jeleń et al., 1997).

Numerous volatile markers for Fusarium infection, identified through SPME/GC-MS, were also detected through OLS/GC-MS. Both methods were, however, not always in agreement. For example, the dynamic OLS captured sesquiterpenoid compounds but no other volatiles, such as $\mathrm{C}_{6}$ hydrocarbons. One reason might be that many short-chain alcohols are poorly retained on activated carbon (Harper, 2000), which was used as adsorbing matrix in OLS, while they are better trapped by polymers (i.e. PDMS-DVB) that were used in the static sampling (Snow, 2000). Employing a multiple layer adsorption in the OLS, as suggested in Liu et al. (2008) and Brancaleoni et al. (1999), or the parallel use of different adsorbing materials could have solved the problem. Another reason for the observed differences between OLS and SPME might be the fact that the SPME sampling was performed on cut and frozen sample material, while the OLS was carried out on live plants. Nevertheless, it has to be stressed that despite these major differences, both methods agreed by $37 \%$ and both confirmed the occurrence of sesquiterpenoid volatiles (including major group of markers) in the headspace of Fusarium infected maize ears.

The majority of volatile markers for Fusarium infection could be detected at an early time point of 4 - $8 \mathrm{dpi}$. At this time stage, a visual detection of fungal infection is hardly possible without opening the ear. Thus, the volatile markers could serve as a tool for an early, nondestructive detection of fungal infection in the field (patent application, University of Goettingen, Germany, DE 101012204237.7).

Sensitive portable devices, such as ion mobility spectrometers (IMS), could possibly be used to transfer the technology to the field or to post-harvest processes (i.e. storage, transport or feed production). The early and fast detection of fusarioses in maize fields by volatile markers and respectively the classification into 'healthy' and 'contaminated' crops, could serve as decision guidance for the application of pesticides (until now not for the field application in Germany) and the selection of a convenient marketing strategy. If highly contaminated fields with fusarioses cannot be used for human and animal consumption (following the regulation for maximum levels of mycotoxins by Commission Regulation (EC) no. 1126/2007), they still might be suitable for energy use. Sensors with a proper sensitivity to distinguish between 
single volatile compounds could be used to monitor the product quality in post-harvest processes, by screening the headspace for target VOCs.

As a whole, the detection of specific volatile markers, as shown in this study, could be a useful risk-management tool for the early and fast detection of Fusarium infection in the field and in post-harvest processes.

\section{Acknowledgements}

We acknowledge all project partners in MykoSensExpert (2010 - 2012), which was funded by the German Ministry of Education and Research and supported by the German Aerospace Centre (DLR). We further acknowledge KWS Saat AG who kindly provided seeds of hybrid maize and Prof. Dr. Jan Schirawski for the supply with dwarf maize seeds. We thank Dr. Tobias Koellner and Sandra Irmisch for their help with the gene expression analysis and for the support with standards for the identification of VOCs. We further acknowledge Dr. Cornelia Herrfurth and Prof. Dr. Ivo Feußner for the analysis of oxylipins. Dr. Richard Splivallo is gratefully acknowledged for the guidance and accomplishment of phytohormone analysis.

\section{Patent application}

A patent on volatile biomarkers for Fusarium infection in maize was filed in March 2012. Patent holder is the University of Goettingen, Germany (DE 101012204237.7). Inventors are Prof. Dr. Petr Karlovsky, Dr. Richard Splivallo and Eva-Maria Becker.

\section{References}

Adejumo TO, Hettwer U, Karlovsky P (2007a) Occurrence of Fusarium species and trichothecenes in Nigerian maize. International Journal of Food Microbiology 116:350-357.

Adejumo TO, Hettwer U, Karlovsky P (2007b) Survey of maize from south-western Nigeria for zearalenone, alpha- and betazearalenols, fumonisin B-1 and enniatins produced by Fusarium species. Food Additives and Contaminants 24:993-1000.
Alencar JW, Craveiro AA, Matos FJA (1984) Kovats' Indices as a Preselection Routine in Mass Spectra Library Searches of Volatiles. Journal of Natural Products 47:890-892.

Anonymus (2007) Commission regulation (EC) no. 1126/2007amending Regulation (EC) No $1881 / 2006$ setting maximum levels for certain contaminants in foodstuffs as regards Fusarium toxins in maize and maize products. Official Journal of the European Union 255:14-17. 
Arthur CL, Pawliszyn J (1990) Solid phase microextraction with thermal desorption using fused silica optical fibers. Analytical Chemistry 62:2145-2148.

Bai G-H, Shaner G (1996) Variation in Fusarium graminearum and Cultivar Resistance to Wheat Scab. Plant Disease 80:975-979.

Bartelt RJ, Wicklow DT (1999) Volatiles from Fusarium verticillioides (Sacc.) Nirenb. and their attractiveness to nitidulid beetles. Journal of Agricultural and Food Chemistry 47:2447-2454.

Bate NJ, Rothstein SJ (1998) C6-volatiles derived from the lipoxygenase pathway induce a subset of defense-related genes. The Plant Journal 16:561-569.

Bennett JW, Klich M (2003) Mycotoxins. Clinical Microbiology Reviews 16:497-516.

Bernasconi ML, Turlings TCJ, Ambrosetti L, et al. (1998) Herbivore-induced emissions of maize volatiles repel the corn leaf aphid, Rhopalosiphum maidis. Entomologia Experimentalis et Applicata 87:133-142.

Boerjesson T, Stoellman U, Adamek P, Kaspersson A (1989) Analysis of volatile compounds for detection of molds in stored cereals. Cereal Chemistry 66 (4): 300-304.

Borsdorf H, Eiceman GA (2006) Ion Mobility Spectrometry: Principles and Applications. Applied Spectroscopy Reviews 41:323375.

Brancaleoni E, Scovaventi M, Frattoni M, et al. (1999) Novel family of multi-layer cartridges filled with a new carbon adsorbent for the quantitative determination of volatile organic compounds in the atmosphere. Journal of Chromatography A 845:317-328.

Brandfass C, Karlovsky P (2008) Upscaled CTABBased DNA Extraction and Real-Time PCR Assays for Fusarium culmorum and $F$. graminearum DNA in Plant Material with Reduced Sampling Error. International Journal of Molecular Sciences 9:2306-2321.

Campagnoli A, Cheli F, Polidori C, et al. (2011) Use of the Electronic Nose as a Screening Tool for the Recognition of Durum Wheat Naturally Contaminated by Deoxynivalenol: A Preliminary Approach. Sensors 11:4899-4916.

D'Mello JPF, Placinta CM, Macdonald AMC (1999) Fusarium mycotoxins: a review of global implications for animal health, welfare and productivity. Animal Feed Science and Technology 80:183-205.

Demyttenaere JC., Moriña RM, De Kimpe N, Sandra P (2004) Use of headspace solidphase microextraction and headspace sorptive extraction for the detection of the volatile metabolites produced by toxigenic Fusarium species. Journal of Chromatography A 1027:147-154.

Dicke M, Baldwin IT (2010) The evolutionary context for herbivore-induced plant volatiles: beyond the 'cry for help'. Trends in Plant Science 15:167-175.

Dickschat JS, Brock NL, Citron CA, Tudzynski B (2011) Biosynthesis of Sesquiterpenes by the Fungus Fusarium verticillioides. ChemBioChem 12:2088-2095.

Dorn B, Forrer H-R, Schuerch S, Vogelgsang S (2009) Fusarium species complex on maize in Switzerland: occurrence, prevalence, impact and mycotoxins in commercial hybrids under natural infection. European Journal of Plant Pathology 125:51-61.

Engelberth J, Alborn HT, Schmelz EA, Tumlinson JH (2004) Airborne signals prime plants against insect herbivore attack. Proceedings of the National Academy of Sciences 101:1781-1785.

Farag MA, Fokar M, Abd H, et al. (2004) (Z)-3Hexenol induces defense genes and downstream metabolites in maize. Planta 220:900-909.

Gelderblom WCA, Kriek NPJ, Marasas WFO, Thiel PG (1991) Toxicity and carcinogenicity of the Fusarium moniliforme metabolite, fumonisin $\mathrm{B} 1$, in rats. Carcinogenesis 12:1247-1251.

Girotti JR, Malbrán I, Lori GA, Juárez MP (2012) Early detection of toxigenic Fusarium graminearum in wheat. World Mycotoxin Journal 5:143-152.

Girotti JR, Malbrán I, Lori GA, Juárez MP (2010) Use of solid phase microextraction coupled to capillary gas chromatographymass spectrometry for screening Fusarium spp. based on their volatile sesquiterpenes. World Mycotoxin Journal 3:121-128.

Glenn AE (2007) Mycotoxigenic Fusarium species in animal feed. Animal Feed Science and Technology 137:213-240.

Goebel C, Feussner I (2009) Methods for the analysis of oxylipins in plants. Phytochemistry 70:1485-1503. 
Goebel C, Feussner I, Rosahl S (2003) Lipid Peroxidation during the Hypersensitive Response in Potato in the Absence of 9Lipoxygenases. Journal of Biological Chemistry 278:52834-52840.

Grob K, Zuercher F (1976) Stripping of Trace Organic Substances from Water Equipment and Procedure. Journal of Chromatography 117: 285 - 294.

Grob K (1973) Organic substances in potable water and in its precursor. Journal of Chromatography A 84:255-273.

Harper M (2000) Sorbent trapping of volatile organic compounds from air. Journal of chromatography. A 885 (1-2): 129-151.

Holopainen JK, Gershenzon J (2010) Multiple stress factors and the emission of plant VOCs. Trends in plant science 15 (3): 176-184.

Huffaker A, Kaplan F, Vaughan MM et al. (2011) Novel acidic sesquiterpenoids constitute a dominant class of pathogen-induced phytoalexins in maize. Plant physiology 156 (4): 2082-2097.

Jeleń HH, Majcher M, Dziadas M (2012) Microextraction techniques in the analysis of food flavor compounds: A review. Analytica Chimica Acta 738: 13-26.

Jeleń HH, Wąsowicz E (1998) Volatile fungal metabolites and their relation to the spoilage of agricultural commodities. Food reviews international 14 (4): 391-426.

Jeleń HH, Latus-Ziętkiewicz D, Wąsowicz E, Kamiński E (1997) Trichodiene as a volatile marker for trichothecenes biosynthesis. Journal of microbiological methods 31 (1): 45-49.

Kaminśki E, Stawicki S, Wąsowicz E (1974) Volatile flavor compounds produced by molds of Aspergillus, Penicillium, and Fungi imperfecti. Applied microbiology 27:1001-1004.

Kesselmeier J, Staudt M (1999) Biogenic volatile organic compounds (VOC): an overview on emission, physiology and ecology. Journal of atmospheric chemistry 33 (1): 23-88.

Koellner TG, Schnee C, Li S, et al. (2008) Protonation of a Neutral (S)- $\beta$-Bisabolene Intermediate Is Involved in (S)- $\beta$ Macrocarpene Formation by the Maize Sesquiterpene Synthases TPS6 and TPS11. Journal of Biological Chemistry 283:20779-20788.
Liu J, Li L, Ning Z, et al. (2008) Multi-layer Cartridges Filled with Multi-Walled Carbon Nanotubes for the Determination of Volatile Organic Compounds in Indoor Air. Analytical Sciences 24:515-519.

Logrieco A, Mulé G, Moretti A, Bottalico A (2002) Toxigenic Fusarium species and mycotoxins associated with maize ear rot in Europe. European Journal of Plant Pathology 108:597-609.

Magan N, Evans P (2000) Volatiles as an indicator of fungal activity and differentiation between species, and the potential use of electronic nose technology for early detection of grain spoilage. Journal of Stored Products Research 36 (4): 319-340.

Matsui K (2006) Green leaf volatiles: hydroperoxide lyase pathway of oxylipin metabolism. Current Opinion in Plant Biology 9:274-280.

Moeller EM, Chełkowski J, Geiger HH (1999) Species-specific PCR Assays for the Fungal Pathogens Fusarium moniliforme and Fusarium subglutinans and their Application to Diagnose Maize Ear Rot Disease. Journal of Phytopathology 147:497-508.

Momin RA, Ramsewak RS, Nair MG (2000) Bioactive Compounds and 1,3-Di [(cis)-9 octadecenoyl]-2-[(cis, cis) $-9,12$ octadecadienoyl] glycerol from Apium graveolens L. Seeds. Journal of Agricultural and Food Chemistry 48:3785-3788.

Mulé G, Susca A, Stea G, Moretti A (2004) A species-specific PCR assay based on the calmodulin partial gene for identification of Fusarium verticillioides, $F$. proliferatum and $F$. subglutinans. European journal of plant pathology 110:495-502.

Nicholson P, Simpson D, Weston G, et al. (1998) Detection and quantification of Fusarium culmorum and Fusarium graminearum in cereals using PCR assays. Physiological and Molecular Plant Pathology 53:17-37.

Nicolaisen M, Supronienė S, Nielsen LK, et al. (2009) Real-time PCR for quantification of eleven individual Fusarium species in cereals. Journal of Microbiological Methods 76:234-240.

Ouyang G, Pawliszyn J (2008) A critical review in calibration methods for solid-phase microextraction. Analytica chimica acta 627 (2): 184-197. 
Perkowski J, Busko M, Chmielewski J, et al. (2008) Content of trichodiene and analysis of fungal volatiles (electronic nose) in wheat and triticale grain naturally infected and inoculated with Fusarium culmorum. International Journal of Food Microbiology 126:127-134.

Piesik D, Lemńczyk G, Skoczek A, et al. (2011) Fusarium infection in maize: Volatile induction of infected and neighboring uninfected plants has the potential to attract a pest cereal leaf beetle, Oulema melanopus. Journal of Plant Physiology 168:1534-1542.

Placinta C, D’Mello JP, Macdonald AM (1999) A review of worldwide contamination of cereal grains and animal feed with Fusarium mycotoxins. Animal Feed Science and Technology 78:21-37.

Rao LJM, Nagalakshimi S, Shankaracharya NB (2000) Studies on chemical and technological aspects of celery (Apium graveolens. Linn) seed. Journal of food science and technology 37 (6): 631-635.

Rostás M, Ton J, Mauch-Mani B, Turlings TCJ (2006) Fungal infection reduces herbivoreinduced plant volatiles of maize but does not affect naive parasitoids. Journal of Chemical Ecology 32:1897-1909.

Rotter BA (1996) Invited review: Toxicology of deoxynivalenol (vomitoxin). Journal of Toxicology and Environmental Health 48:1-34.

Sabater-Jara AB, Almagro L, Belchí-Navarro S, et al. (2010) Induction of sesquiterpenes, phytoesterols and extracellular pathogenesis-related proteins in elicited cell cultures of Capsicum annuum. Journal of Plant Physiology 167:1273-1281.

Schmelz, EA,. Engelberth J, Tumlinson JH, et al. (2004) The use of vapor phase extraction in metabolic profiling of phytohormones and other metabolites. The Plant Journal 39 (5): 790-808.

Schnee C, Koellner TG, Held M, et al. (2006) The products of a single maize sesquiterpene synthase form a volatile defense signal that attracts natural enemies of maize herbivores. Proceedings of the National Academy of Sciences 103:1129-1134.

Schnuerer J, Olsson J, Boerjesson T (1999) Fungal Volatiles as Indicators of Food and Feeds Spoilage. Fungal Genetics and Biology 27 (2-3): 209-217.
Siegel S, Castellan NJ (1988) Nonparametric Statistics for the Behavioral Sciences. 2. Edition. Singapore: McGraw-Hill.

Snow NH (2000) Solid-phase micro-extraction of drugs from biological matrices. Journal of chromatography. A 885 (1-2): 445-455.

Sokovic M, Stojković D, Glamočlija J, et al. (2009) Susceptibility of pathogenic bacteria and fungi to essential oils of wild Daucus carota. Pharmaceutical Biology 47:38-43.

Stewart-Jones A, Poppy GM (2006) Comparison of Glass Vessels and Plastic Bags for Enclosing Living Plant Parts for Headspace Analysis. Journal of Chemical Ecology 32:845-864.

Tholl, D, Boland W, Hansel A, et al. (2006) Practical approaches to plant volatile analysis. The Plant Journal 45 (4): 540560.

Tuetun B, Choochote W, Pongpaibul Y, et al. (2008) Celery-based topical repellents as a potential natural alternative for personal protection against mosquitoes. Parasitology Research 104:107-115.

Turner APF, Magan N (2004) Electronic noses and disease diagnostics. Nature Reviews Microbiology 2 (2): 161-166.

Turner AS, Lees AK, Rezanoor HN, Nicholson P (1998) Refinement of PCR-detection of Fusarium avenaceum and evidence from DNA marker studies for phenetic relatedness to Fusarium tricinctum. Plant Pathology 47:278-288.

Utermark J, Karlovsky P (2007) Role of Zearalenone Lactonase in Protection of Gliocladium roseum from Fungitoxic Effects of the Mycotoxin Zearalenone. Applied and Environmental Microbiology 73(2): 637-642.

Vas G, Vékey K (2004) Solid-phase microextraction: a powerful sample preparation tool prior to mass spectrometric analysis. Journal of Mass Spectrometry 39:233-254.

Vautz W, Zimmermann D, Hartmann M, et al. (2006) Ion mobility spectrometry for food quality and safety. Food Additives and Contaminants 23:1064-1073. 
Visentin I, Montis V, Doell K, Alabouvette C, Tamietti G, Karlovsky P, Cardinale F (2012) Transcription of genes in the biosynthetic pathway for fumonisin mycotoxins is epigenetically and differentially regulated in the fungal maize pathogen Fusarium verticillioides. Eukaryotic Cell 11:252-259.

Wenke K, Kai M, Piechulla B (2009) Belowground volatiles facilitate interactions between plant roots and soil organisms. Planta 231:499-506.

Zadoks JC, Chang TT, Konzak CF (1974) A decimal code for the growth stages of cereals. Weed Research 14:415-421.

Zinedine A, Soriano JM, Moltó JC, Mañes J (2007) Review on the toxicity, occurrence, metabolism, detoxification, regulations and intake of zearalenone: An oestrogenic mycotoxin. Food and Chemical Toxicology 45:1-18. 


\title{
3 Volatile Biomarkers for the Fusarium Infection of Wheat (Triticum aestivum L.) Ears
}

\begin{abstract}
Fusarium species cause huge economic losses in maize and small-grain cereals all over the world. The Fusarium head blight (FHB) of wheat is caused by a species complex of F. graminearum, F. culmorum, F. avenaceum and other species. FHB is often accompanied with the accumulation of toxic secondary metabolites of fungi, named mycotoxins, such as deoxynivalenol (DON) and nivalenol (NIV). These metabolites exert negative effects on humans and animals (i.e. immune-suppression).

In the present study, we investigated the emissions of volatile organic compounds (VOCs) from Fusarium infected summer wheat ears (including F. graminearum, F. avenaceum and $F$. poae single and mixed infections) under greenhouse conditions by SPME/GC-MS. We analysed the mycotoxin production by HPLC-MS and fungal biomass by species-specific real-time PCR. Observations were carried out at 21 days post-inoculation (dpi) as well as in time series (TS) from 7 to 21 dpi (TS only with $F$. graminearum and $F$. avenaceum).

We detected a set of 13 volatile biomarkers for the infection of wheat ears with Fusarium spp. The set included common volatiles already reported in fungi and plants, such as (E)-2-hexenal and 3-octanone, but also two unidentified sesquiterpenes (Kováts' retention indice (KI) 1421 and KI 1467). These terpenoid markers were only released from Fusarium infected material and serve, therefore, as qualitative markers for Fusarium infection. Apart from the volatile analysis, we found abnormally high concentrations of mycotoxins, especially trichothecenes, in infected samples. Furthermore, we observed a suppression of F. avenaceum DNA and enniatin production upon mixed infection with $F$. graminearum.
\end{abstract}

The present set of volatile biomarkers may be transferred to the field (i.e. screening for relevant volatiles with portable detectors) and could serve as fast decision guidance for plant protection strategies. 


\subsection{Introduction}

Pathogenic Fusarium spp. cause severe problems in the production of small-grain cereals, especially wheat (Triticum aestivum L.) all over the world (Goswami \& Kistler, 2004; McMullen et al., 1997). The typical discolouration of the infected ear, described as Fusarium head blight (FHB), is mainly caused by a species complex of F. graminearum Schwabe, F. culmorum (W. G. Smith) Sacc. and F. avenaceum (Fr.), but other species may be involved (Parry et al., 1995). The infection of cereals with Fusarium spp. causes huge economic losses and a reduction of product quality by the contamination with toxic secondary fungal metabolites (mycotoxins). Among this group of Fusarium toxins the sesquiterpenoid trichothecenes, such as deoxynivalenol (DON) and nivalenol (NIV), are of outstanding importance (Bennett \& Klich, 2003; D’Mello et al., 1999; Placinta et al., 1999). These metabolites can cause multiple toxic effects in humans and animals. Moreover, an estrogenic toxin, named zearalenone (ZEN), with strong influences in animal production is produced by some Fusarium spp. (Doell \& Daenicke, 2011; Zinedine et al., 2007; Kuiper-Goodman et al., 1987). Hence, the European Commission established thresholds for the most common Fusarium toxins (Commission Regulation (EC) no. 1126/2007).

To prevent the transfer of contaminated grains to the food chain is a major task for farmers, traders and fabricants, but also governmental authorities (Miller, 2008; Morgavi \& Riley, 2007). Up to now, the exact determination of Fusarium contamination is conducted by time-consuming and expensive methods, such as quantitative real-time PCR for the specific determination of fungal biomass (Niessen, 2007) and liquid chromatography mass spectroscopy (LC-MS) for the evaluation of toxin contamination (Krska et al., 2008). These methods do not provide a fast decision guidance for the production chain, since they require a time-consuming sample preparation. A novel approach for the detection of fungi in agricultural commodities is the analysis of volatile organic compounds (VOCs) as fast indicators for fungal spoilage (Schnuerer et al., 1999). The most common technique for volatile analysis is gas chromatography mass spectrometry (GC-MS) (Tholl et al. 2006), but recent studies report the use of electronic noses (E-noses) or other transportable devices to discriminate between contaminated and non-contaminated material (Campagnoli et al., 2011; Eifler et al., 2011; Presicce et al., 2006).

Jeleń et al. (1997) described the volatile isoprenoid trichodiene as a specific marker for trichothecene syntheses of toxigenic Fusarium species. The outstanding role of trichodiene 
was later supported by other studies (Girotti et al., 2012; Perkowski et al., 2008). Apart from this prominent compound, other sesquiterpenes, but also alcohols and aldehydes were reported as important volatiles emitted by Fusarium infected cereal grains (Piesik et al., 2011; Girotti et al., 2010; Presicce et al., 2006; Boerjesson et al., 1989).

So far, most studies investigating volatile emissions upon Fusarium infection were carried out on sterile grains or artificial media (Girotti et al., 2010; Presicce et al., 2006; Demyttenaere et al., 2004; Jeleń et al., 1997; Boerjesson et al., 1989). $\quad$ Only Girotti et al. (2012) and Perkowski et al. (2008) monitored volatiles from infected wheat trials in the field. Both groups were mainly referring to trichodiene as marker for infection. Currently, there is a lack of knowledge about the emissions of the diverse spectrum of volatiles from living wheat plants, infected with important Fusarium species, and the need to establish a specific set of volatile markers for an infection under natural conditions. In future, this set may provide a possible tool for Fusarium infection management and decision guidance for fungicide application.

In the present study, we intended to investigate, if a set of early volatile biomarkers is available upon infection of wheat plants by major toxigenic Fusarium species originating from different climatic regions ( $F$. graminearum, $F$. avenaceum and $F$.poae) (spatial distributions and climatic conditions, favoured by different Fusarium spp., were discussed by Bottalico \& Perrone (2002) and Parry et al. (1995)). Moreover, we wanted to observe the temporal emission of volatiles and to link our findings with severity of infection and further methods commonly used to screen for the presence of Fusarium (i.e. fungal biomass determined by quantitative PCR and concentrations of mycotoxins determined by LC-MS analysis). 


\subsection{Materials and Methods}

\subsubsection{Plant Material and Growth Conditions}

A commercial summer wheat cultivar ('Taifun', moderate susceptible to FHB, obtained from KWS Saat AG, Einbeck, Germany) was grown under greenhouse conditions in two independent repetitions. Temperature was set to $26 \pm 5{ }^{\circ} \mathrm{C}$. Light regime was set to $16 \mathrm{~h}$ day / $8 \mathrm{~h}$ night using mercury vapour lamps (6600 lux). Relative humidity (RH) was $58 \pm 16 \%$. Four seeds per plastic pot $(11 \times 11 \times 12 \mathrm{~cm})$ were sown in mixed soil consisting of $70 \%$ plant substrate (Fruhstorfer Erde, Typ 25, HAWITA Group, Vechta, Germany) and $30 \%$ sand (0$2 \mathrm{~mm}$ granularity). Plants were bottom-irrigated with tap water as required. Number of wheat plants per pot was reduced to two within 6-8 days after germination. The wheat was provided with a macronutrient fertilizer as described for maize (see chapter 2, section 2.2.1). Side shoots were continuously removed until harvest.

\subsubsection{Fungal Material and Infection}

If not stated otherwise, the details on fungal strains are given in the descriptions for maize infection in chapter 2 (section 2.2.2 and Tab. 2.1). The infections of wheat ears were carried out with two strains of $F$. graminearum (labelled as FG1 and FG2), one strain of F. avenaceum (labelled as FA) and one strain of F. poae (labelled as FP: strain F448, obtained from A. Prodi, University of Bologna, Italy). Each strain was used as single treatment as well as in mixed infection treatments with MIX A, being a mixture of FG1 and FA spores, and MIX B, consisting of FG1 and FP spores.

Fungal spores were produced following a modified protocol of Bai \& Shaner (1996). The modifications included one step of pre-culturing on mung bean medium (mung beans obtained from Alnatura GmbH, Bickenbach, Germany). Spore suspensions of the pre-culture were transferred to the new medium. After 5 days of incubation, the spore suspensions were centrifuged, decanted and spores were re-dissolved in sterile glycerol (15\%, Carl Roth, Karlsruhe, Germany). Suspensions were stored at $-70{ }^{\circ} \mathrm{C}$ and germination tests were carried out regularly on potato dextrose agar (PDA, Carl Roth, Karlsruhe, Germany) to check for the proper viability of spores. 
For the production of inoculum, spores were counted with a Thoma chamber $\left(0.0025 \mathrm{~mm}^{2}\right)$ and dissolved in sterile tap water to obtain concentrations of $2 \times 10^{5}$ spores $\mathrm{mL}^{-1}$. In case of MIX A and MIX B, spores of each strain were produced separately and mixed before use. A volume of $20 \mu \mathrm{L}$ Tween ${ }^{\circledR} 20$ (Applichem, Darmstadt, Germany) was transferred to each spore suspension (prepared in aliquots of $12 \mathrm{~mL}$ ). The control treatment was carried out with water and Tween ${ }^{\circledR} 20$. Prepared inoculum was stored at $4{ }^{\circ} \mathrm{C}$ for a maximum of 3 days.

Infections of wheat ears were carried out by spray inoculation (described by Miedaner et al., 2003) at main flowering stage (stage BBCH 65 as established by Zadoks et al. (1974)). The time point was determined individually by a daily observation of emerging anthers. Spore suspensions were transferred to spray diffusers (Carl Roth, Karlsruhe, Germany, $20 \mathrm{~mL}$ total volume, $0.05 \mathrm{~mL}$ per dose). Each ear was treated with a volume of $1.5 \mathrm{~mL}$ of spore suspension. For this purpose, 15 spray doses were applied on each side of the ear with a distance of $10 \mathrm{~cm}$. The wet ear was subsequently covered for $48 \mathrm{~h}$ with a transparent plastic bag (DM, Karlsruhe, Germany). Prior to use, the bag was moistened with water at the inner surface. If the inoculation time points of two plants growing in one pot were different, the second ear was covered with a plastic bag. To exclude cross-contamination in greenhouse chamber, each ear was covered with a cylindrical PE-tube until sampling.

\subsubsection{Assessment of Disease Severity}

Disease severity (DS) was assessed individually after 7, 14 and 21 days post-inoculation (dpi). The parameter was calculated as described in formula [1] (see below). The area under disease progress curve (AUDPC) was calculated as described in Simko \& Piepho (2012). The equation is given in [2] (see below).

[1] $D S=\frac{\text { number of infected spikelets x percentage of infected surface }}{\text { total number of spikelets per ear }}$

[2] $\quad A U D P C=\left[D S_{1} \times \frac{T P_{2}-T P_{1}}{2}\right]+\left[\sum_{i=2}^{n-1}\left(D S_{i} \times \frac{T P_{i+1}-T P_{i-1}}{2}\right)\right]+\left[D S_{n} \times \frac{T P_{n}-T P_{n-1}}{2}\right]$

AUDPC $=$ Area under disease progress curve

DS = Disease Severity

$\mathrm{TP}=$ Time point of assessment 


\subsubsection{Sample Preparation and Collection of Volatile Molecules}

If not stated otherwise, the used materials and preparative steps in the following descriptions are the same as for the maize experiment (references are given in chapter 2, sections 2.2.5 2.2.6).

A static sampling of volatiles was carried out with head space solid-phase microextraction (HS-SPME). For this purpose, wheat ears were harvested at $21 \mathrm{dpi}$ (end point harvest) for all infection treatments as well as in time series for FG1 and FA treatments after 7, 14 and 21 dpi. Experimental schedule of wheat trials is given in Tab. 3.1. Ears were collected, weighed and DS was estimated. For further analysis, each sample (each replicate) was generated by pooling of 4 ears. Hence, for HS-SPME, all spikelets from one side of an ear (4 ears per replicate) were transferred to a $20 \mathrm{~mL}$ SPME vial, which was thereafter closed air tight with a screw cap equipped with a silicone-polytetrafluoroethylene (PTFE) septum (both obtained from VWR, Darmstadt, Germany) and stored at $-20{ }^{\circ} \mathrm{C}$ prior to analysis of volatiles. The remaining spikelets from the opposite side of the ears were pooled, oven-dried at $45^{\circ} \mathrm{C}$ for 7 - 9 days and ground with a ball mill (Retsch, Haan, Germany). This material was used for supplemental analysis, such as real-time PCR for the determination of fungal biomass in ear tissue (section 3.2.7) and mycotoxin analysis by liquid chromatography (section 3.2.6). Volatile molecules were extracted with HS-SPME and analyzed by GC-MS (SPME/GC-MS) as described for maize (see chapter 2, sections 2.2.6 and 2.2.8).

Tab. 3.1 Experimental schedule for summer wheat trials

\begin{tabular}{|c|c|c|c|c|}
\hline \multirow{2}{*}{$\begin{array}{l}\text { Treatment/ } \\
\text { Fusarium spp. }\end{array}$} & \multirow[b]{2}{*}{ Abbreviation } & \multirow{2}{*}{$\begin{array}{c}\text { Experiment } 1 \\
\text { SPME }^{1}\end{array}$} & \multicolumn{2}{|c|}{ Experiment 2} \\
\hline & & & SPME $^{1}$ & $\begin{array}{l}\text { Time series }^{2} \\
\text { (SPME) }\end{array}$ \\
\hline F. graminearum & FG1 & $\mathrm{n}=8$ & $\mathrm{n}=5$ & 3 time points, $n=3$ \\
\hline F. graminearum & $\mathrm{FG} 2$ & $\mathrm{n}=8$ & $\mathrm{n}=5$ & - \\
\hline F. avenaceum & FA & $\mathrm{n}=8$ & $\mathrm{n}=5$ & 3 time points, $n=3$ \\
\hline F. poae & FP & $\mathrm{n}=8$ & $\mathrm{n}=5$ & - \\
\hline$(F G 1+F A)$ & MIX A & $\mathrm{n}=8$ & $\mathrm{n}=5$ & - \\
\hline$(F G 1+F P)$ & MIXB & $\mathrm{n}=8$ & $\mathrm{n}=5$ & - \\
\hline
\end{tabular}

${ }^{1}$ Solid-phase microextraction (SPME) at time point 21 days post-inoculation (dpi)

${ }^{2}$ SPME at time points 7,14 and $21 \mathrm{dpi}$ 


\subsubsection{In vitro Assay with Autoclaved Wheat and Rice Kernels}

Volatile profile of $F$. graminearum (FG1), F. avenaceum (FA) and $F$. poae (FP) cultures on sterile rice and wheat medium were investigated. The procedure followed the descriptions for maize $(n=3$, chapter 2 , section 2.2.10). Non-inoculated material of summer wheat was collected from greenhouse trials, checked for fungal infection by quantitative real-time PCR (data not shown) and used as wheat medium for fungal cultures.

\subsubsection{Mycotoxin Extraction and Analysis}

Mycotoxins were extracted from aliquots of $300 \mathrm{mg}$ homogenized wheat flour according to the protocols presented in chapter 2 (section 2.2.12).

\subsubsection{Quantification of Fungal DNA}

Similarly to maize, the concentration of fungal DNA was assessed by real-time PCR and used as parameter for fungal biomass. Fungal DNA was extracted from $100 \mathrm{mg}$ wheat flour with the CTAB method as described in Brandfass \& Karlovsky (2008). The preparation of DNA standards in wheat matrix and quantitative real-time PCR analysis followed the descriptions already presented in chapter 2 (section 2.2.13). The PCR assay for $F$. poae was optimized by Dastjerdi (not yet published).

\subsubsection{Data Processing and Statistical Analysis}

Processing of GC-MS data was performed as described for maize (chapter 2, section 2.2.18), including the calculation of Kováts' retention indices (KI) and comparison with mass spectral libraries. Because of possible cross-contamination of the wheat samples, all samples were screened by Fusarium species-specific real-time PCR. Samples showing an infection with Fusarium species that were different from the species used for inoculation were excluded from the analysis. Consequently, samples of control treatment were excluded, if the PCR analysis revealed a fungal infection. Volatile compounds were selected for the set of biomarkers, if they were statistically confirmed by both experiments, (Kruskal-Wallis test, $\mathrm{p}=0.01)$.

Further quantitative data, i.e. toxin concentration and fungal biomass, were averaged within infection treatments. Single results below the limit of quantification (LOQ) were set to 0. If 
not stated otherwise, results are presented for one out of two independent wheat experiments under greenhouse conditions. 


\subsection{Results}

\subsubsection{Selected Volatile Markers for the Fusarium Infection of Wheat}

The two independent greenhouse experiments with summer wheat revealed high variations in disease development (DS was remarkably higher in one out of two experiments among all infection treatments). Thus, only common volatile compounds, statistically confirmed by both experiments, were selected for the set of biomarkers.

The level of 13 volatiles, identified by SPME/GC-MS, significantly differed between Fusarium infected and non-infected summer wheat plants. This set of biomarkers included ketons, aldehydes, alcohols and terpenoid compounds. The concentrations of volatiles were remarkably lower than in maize (see chapter 2, section 2.3). Qualitative and quantitative differences between healthy plants and Fusarium infected plants were detected. An overview about identification, quantities and statistical results for both independent experiments are presented in Tab. 3.2. Volatile (E)-2-hexenal, 2-heptanol, 1-octen-3-ol and an unknown compound (KI 1516) were most abundant in uninfected plants. Compounds, detected in single infections, were also detected in mixed infection treatments, although mixed treatments seemed to be dominated by $F$. graminearum related volatiles. Two sesquiterpenoid compounds (m/z: 204, KI 1421, KI 1467) that were not identified by mass-spectral libraries, appeared in all infection treatments with the exception of $F$. poae treatment. Their mass spectra are presented in Fig. 3.1 A.

Quantitative differences in VOCs emission between infection treatments, given as simple colour code based on normalized data, are illustrated in Fig. 3.1 B. Because of overlapping peaks and mass fragments, it was not possible to distinguish between 2-pentyl-furan, which was also present in maize, but not identified as marker, and 2-octanone.

As previously described for maize (chapter 2), marker compounds were not detected under in vitro conditions on rice and wheat medium. 
Tab. 3.2 Normalized peak areas of 13 selected volatile markers for Fusarium infection in summer wheat ears after 21 days post-inoculation (dpi)

\begin{tabular}{|c|c|c|c|c|c|c|c|c|c|c|c|c|c|c|c|}
\hline \multirow{3}{*}{$\mathrm{KI}_{\text {calc }}{ }^{2}$} & \multirow{3}{*}{$I^{3}$} & \multicolumn{14}{|c|}{ Mean peak area, normailized by total ion chromatogram $(\mathrm{TIC}) \pm$ standad error $^{1}$} \\
\hline & & \multicolumn{7}{|c|}{ Wheat experiment $1(n=8)$} & \multicolumn{7}{|c|}{ Wheat experiment $2(n=5)$} \\
\hline & & CONT & FA & FG1 & FG2 & FP & MIX A & MIX B & CONT & FA & FG1 & FG2 & FP1 & MIX A & MIX B \\
\hline 711 & 2-pentanone & $1.46 \pm 0.37$ & $2.53 \pm 0.21$ & $3.86 \pm 0.18$ * & $5.70 \pm 0.39$ * & $2.57 \pm 0.45$ & $3.72 \pm 0.35$ * & $3.71 \pm 0.38$ * & $2.01 \pm 0.65$ & $0.65 \pm 0.46$ & $3.59 \pm 0.46$ & $3.04 \pm 0.53$ & $2.84 \pm 0.17$ * & $2.24 \pm 0.15$ & $2.76 \pm 0.41$ \\
\hline 754 & 2-hexanone & $0.03 \pm 0.02$ & $0.15 \pm 0.02$ & $0.19 \pm 0.02$ * & $0.24 \pm 0.03$ * & $0.12 \pm 0.04$ & $0.16 \pm 0.04$ * & $0.22 \pm 0.02 *$ & $0.11 \pm 0.11$ & - & $0.35 \pm 0.05$ * & $0.38 \pm 0.07$ * & - & $0.31 \pm 0.08$ & $0.16 \pm 0.06$ \\
\hline 799 & 2-hexenal, (E)- & $1.05 \pm 0.33$ & $0.28 \pm 0.17$ * & $0.01 \pm 0.01$ * & $0.06 \pm 0.04$ * & $0.51 \pm 0.32$ * & $0.08 \pm 0.06$ * & $0.19 \pm 0.19$ * & $0.21 \pm 0.08$ & - & - & - & - & - & - \\
\hline 831 & 2-heptanone & $0.32 \pm 0.04$ & $0.78 \pm 0.16$ & $1.22 \pm 0.11$ * & $1.14 \pm 0.10$ * & $0.52 \pm 0.08$ & $1.08 \pm 0.19$ * & $1.06 \pm 0.12$ * & $0.28 \pm 0.09$ & $0.05 \pm 0.03$ & $1.29 \pm 0.33$ * & $0.69 \pm 0.08$ & $0.17 \pm 0.06$ & $1.18 \pm 0.18$ * & $0.64 \pm 0.12$ \\
\hline 842 & 2-heptanol & $0.98 \pm 0.33$ & $0.13 \pm 0.13$ * & - & - & $0.40 \pm 0.27$ & - & - & $0.16 \pm 0.05$ & - & - & - & - & - & - \\
\hline 919 & no ID & $0.08 \pm 0.01$ & $0.23 \pm 0.07$ * & $0.10 \pm 0.01$ * & $0.23 \pm 0.07$ * & $0.05 \pm 0.02$ & $0.24 \pm 0.12$ * & $0.19 \pm 0.12$ & - & $0.07 \pm 0.05$ & $0.36 \pm 0.12$ * & $0.14 \pm 0.07$ & - & $0.27 \pm 0.04$ * & $0.14 \pm 0.06$ \\
\hline 925 & 1-octen-3-ol & $0.50 \pm 0.07$ & $0.34 \pm 0.12$ & $0.10 \pm 0.01$ * & $0.28 \pm 0.09$ & $0.14 \pm 0.07$ * & $0.36 \pm 0.20$ * & $0.36 \pm 0.27$ * & $0.33 \pm 0.14$ & $0.05 \pm 0.05$ & $0.27 \pm 0.08$ & $0.07 \pm 0.07$ * & - & $0.17 \pm 0.04$ & $0.04 \pm 0.04$ * \\
\hline 933 & 3-octanone & $0.11 \pm 0.02$ & $0.76 \pm 0.32$ & $1.08 \pm 0.17$ * & $1.68 \pm 0.48$ * & - & $0.67 \pm 0.30$ & $1.04 \pm 0.42$ & $0.36 \pm 0.14$ & $0.05 \pm 0.04$ & $9.55 \pm 2.10$ * & $3.72 \pm 0.78$ & $0.05 \pm 0.02$ & $7.90 \pm 0.69$ * & $5.35 \pm 2.08$ * \\
\hline 936 & furan, 2-pentyl- / 2-octanone & $0.43 \pm 0.08$ & $1.24 \pm 0.62$ & $0.77 \pm 0.11$ * & $0.94 \pm 0.15$ * & $0.24 \pm 0.03$ & $0.95 \pm 0.22$ * & $0.62 \pm 0.13$ & $0.62 \pm 0.40$ & $0.27 \pm 0.06$ & $3.78 \pm 0.48$ * & $2.64 \pm 0.18$ & $0.20 \pm 0.10$ & $3.55 \pm 0.35$ * & $2.78 \pm 0.56$ * \\
\hline 945 & 3-octanol & $0.05 \pm 0.04$ & $0.09 \pm 0.05$ & $0.07 \pm 0.01$ * & $0.12 \pm 0.03$ * & $0.01 \pm 0.01$ & $0.12 \pm 0.04$ * & $0.03 \pm 0.01$ & - & $0.02 \pm 0.02$ & $0.22 \pm 0.10$ & $0.13 \pm 0.06$ & - & $0.33 \pm 0.04$ * & $0.09 \pm 0.06$ \\
\hline 1421 & SQT unknown & - & $0.05 \pm 0.02$ * & $0.04 \pm 0.01$ * & $0.01 \pm 0.01$ & - & $0.04 \pm 0.01$ * & $0.01 \pm 0.00$ & - & $0.10 \pm 0.01$ * & $0.04 \pm 0.04$ & - & - & $0.06 \pm 0.03$ & $0.03 \pm 0.03$ \\
\hline 1467 & SQT unknown & - & $0.05 \pm 0.02$ * & $0.03 \pm 0.01$ * & $0.04 \pm 0.01$ * & $0.06 \pm 0.02$ * & $0.04 \pm 0.02$ * & $0.03 \pm 0.01$ & - & - & $0.39 \pm 0.10$ * & $0.27 \pm 0.06$ * & $0.05 \pm 0.05$ & $0.62 \pm 0.24$ * & $0.20 \pm 0.06$ * \\
\hline 1516 & no ID & $0.23 \pm 0.06$ & - & - & & - & $0.01 \pm 0.01$ * & $0.01 \pm 0.01$ * & $0.08 \pm 0.02$ & - & - & - & - & - & - \\
\hline
\end{tabular}

${ }^{1}$ Analysis of HS-SPME samples by GC-MS, significant differences to control treatment are labelled with "*' (statistic: Kruskal-Wallis test, $p=0.01$ ), Treatments: CONT = water treatment;

$\mathrm{FA}=F$. avenaceum; $\mathrm{FG} 1$ and $\mathrm{FG} 2=F$. graminearum strains, $\mathrm{FP}=F$. poae, $\mathrm{MIX} \mathrm{A}=\mathrm{FG} 1$ and $\mathrm{FA}, \mathrm{MIX} \mathrm{B}=\mathrm{FG} 1$ and $\mathrm{FP}$ (further information on strains is provided in chapter 2, Tab. 2.1)

2 Calculated Kováts' retention index, based on homologous series of $n$-alkanes

${ }^{3}$ Chemical identity, obtained from mass spectral libaries 
A
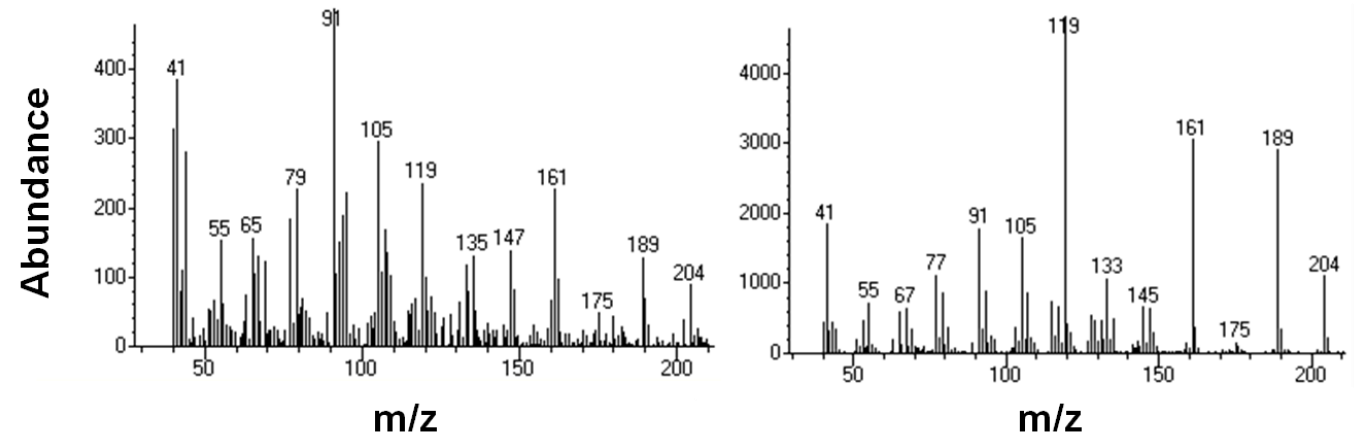

B
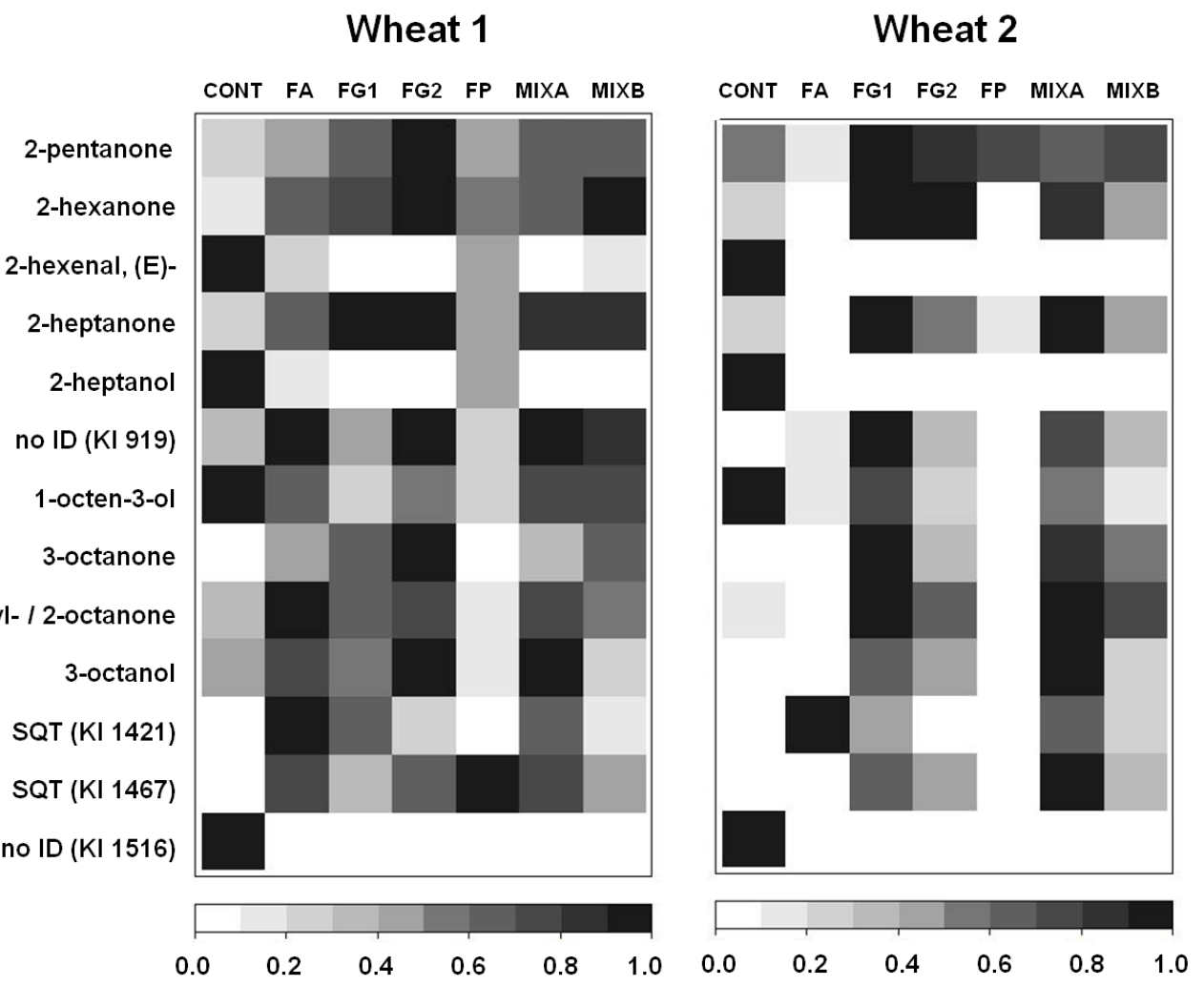

Fig. 3.1 Identified volatile markers for Fusarium infection in wheat, (A) mass spectra of unknown sesquiterpenes (SQT) (m/z = mass-to-charge ratio), calculated Kováts' indices $(\mathrm{KI})$ are presented in brackets, (B) Distribution of selected volatile markers for Fusarium infection in wheat at time point $21 \mathrm{dpi}$, based on normalized GC-MS-data (peak areas, normalized by total ion chromatogram (TIC), and mean values normalized by maximum within marker and experiment), color coding is specified in legend $(1=$ maximum, $0=$ not detected), Abbreviations: Wheat $1=$ first wheat experiment $(n=8)$, Wheat $2=$ second wheat experiment $(n=5)$, infection treatments CONT, FA, FG1, FG2, FP, MIX A and MIX $\mathrm{B}$ are described in Tab. 3.2 


\subsubsection{Volatile Terpenoids Released Upon Moderate Infection with $F$. avenaceum and F.poae}

As mentioned before, estimated disease severities at time point $21 \mathrm{dpi}$ varied among both trials. Mean DS \pm standard deviation observed for FA infection was $33 \pm 12 \%$ in one trial and $71 \pm 8 \%$ in the other trial. Mean DS of FP infection was $11 \pm 10 \%$ in one and $41 \pm 7 \%$ in the other trial. Two monoterpenes (m/z: 136), appearing exclusively upon $F$. avenaceum infection and two sesquiterpenes (SQTs) that were only present in F. poae infected spikelets, were detected in both trials. They were however identified as statistically significant infection markers only in one out of two experiments (only in the case where estimated disease severities were low). Mass spectra of these terpenoids are presented in Fig. 3.2.

\section{F. avenaceum}

Unknown MT (KI 962)

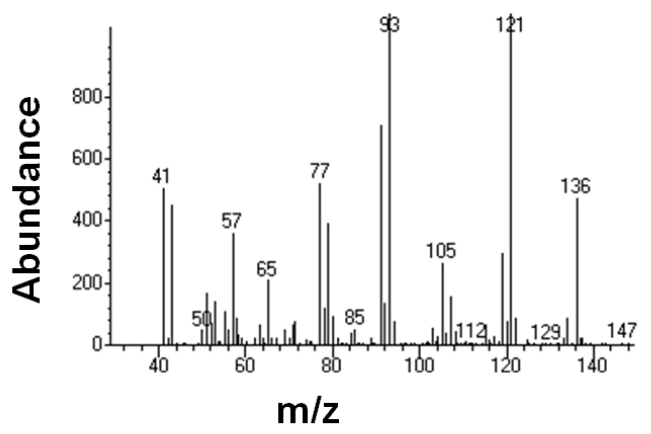

Unknown MT (KI 976)

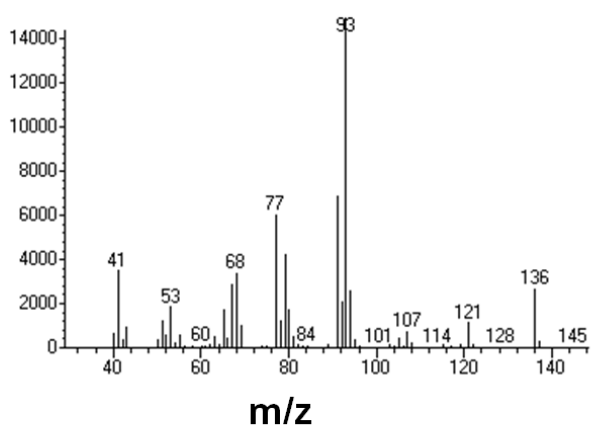

F. poae

Unknown SQT (KI 1541)

Unknown SQT (KI 1578)
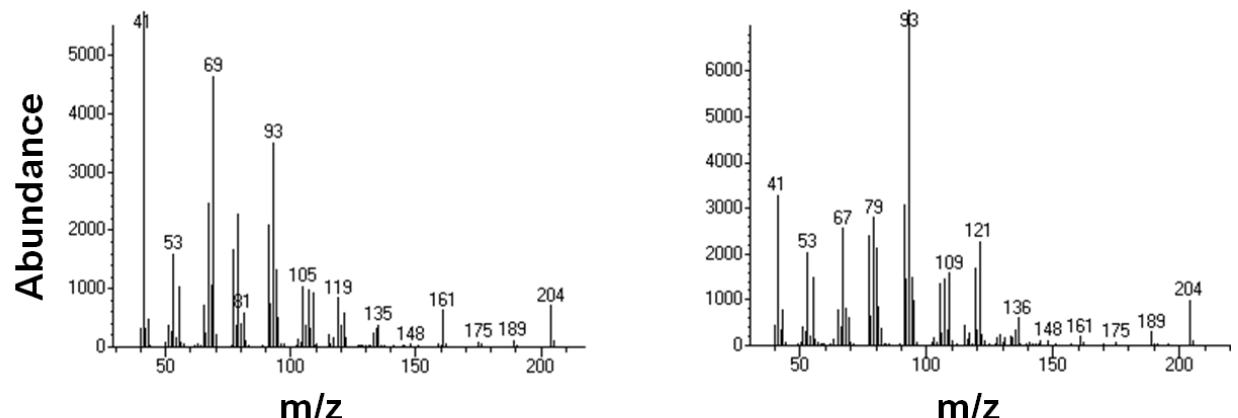

$\mathrm{m} / \mathrm{z}$

Fig. 3.2 Mass spectra of unknown monoterpenes (MT), detected upon $F$. avenaceum infection of wheat and unknown sesquiterpenes (SQT), detected upon F. poae infection; terpenoid volatiles were detected in both experiments, but not statistically confirmed as common markers by Kruskal-Wallis test $(p=0.01)$, mass-to-charge ratio $=\mathrm{m} / \mathrm{z}$, calculated Kovàts' indices $(\mathrm{KI})$ are presented in brackets 


\subsubsection{Time Series - Temporal Release of Volatile Compounds from Fusarium Infected Wheat Ears}

At time point $7 \mathrm{dpi}, 10$ out of 13 selected volatiles were present in both infection treatments with $F$. graminearum strain FG1 and $F$. avenaceum strain FA. Control signals (E)-2-hexenal and unknown compound (KI 1516) were absent in time series with infected ears. The unknown sesquiterpene (KI 1421) was present from 7 dpi until 21 dpi in FA treatment and appeared from 14 dpi upon infection with FG1. Unknown SQT (KI 1467) was present in FG1 infected tissue at time point $21 \mathrm{dpi}$ and absent in F. avenaceum treatment. 2-Heptanol, previously described with highest signal intensities in the control treatment, was absent at 21 dpi, but present at earlier time points. Fig. 3.3 illustrates temporal emission of 6 selected volatile markers for Fusarium infection of wheat ears.

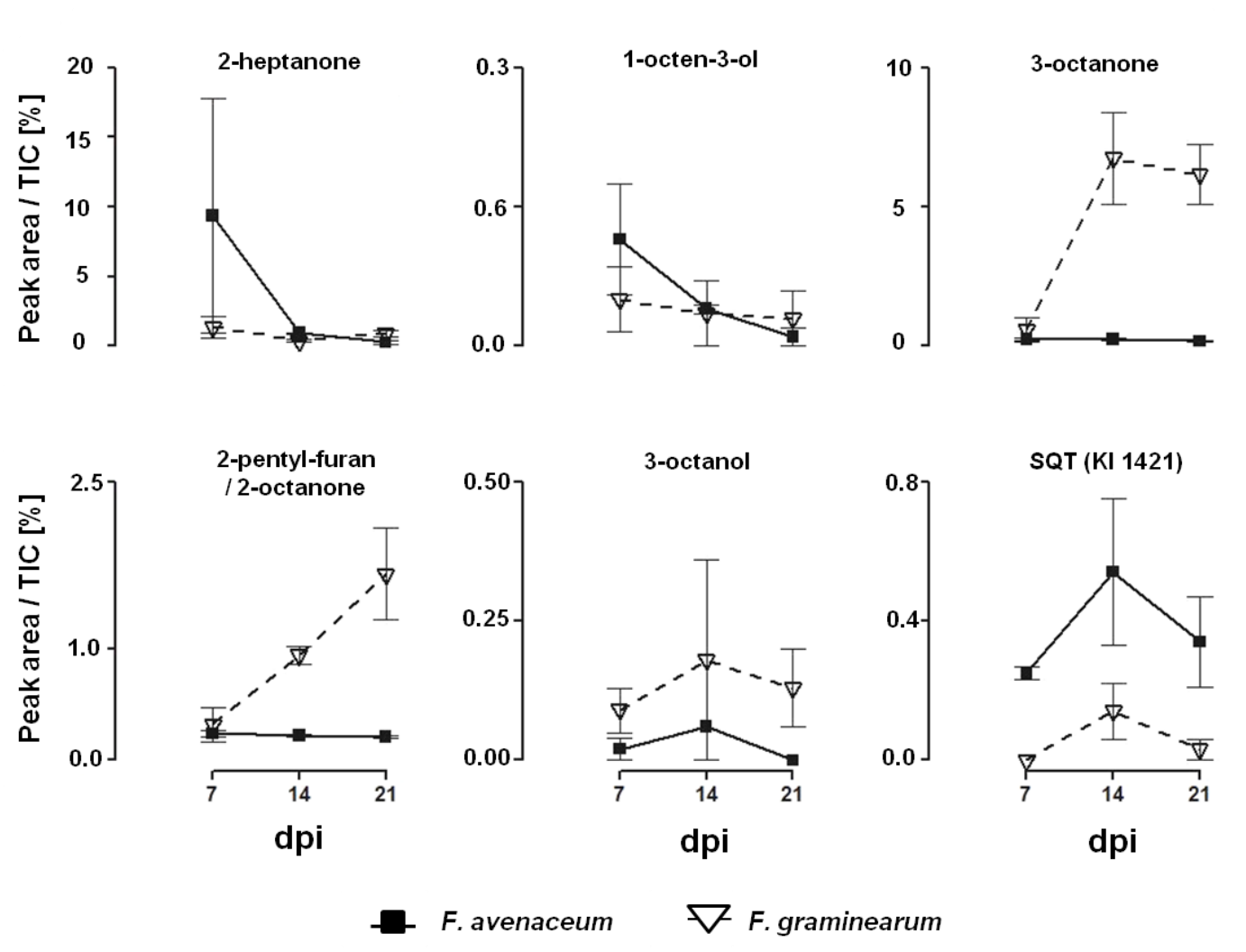

Fig. 3.3 Temporal emission of 6 selected volatile markers in Fusarium infected wheat ears over 3 time points from 7 to 21 days post-inoculation (dpi), data given as peak area normalized by total ion chromatogram (TIC) \pm standard error, analysis was performed with SPME/GC-MS, calculated Kováts' indice (KI) of unknown sesquiterpene (SQT) is presented in brackets, $n=3$ 


\subsubsection{Disease Progress}

Disease symptoms on artificially infected wheat ears were present from time point $7 \mathrm{dpi}$ and intensified over the time. Estimates of disease severity (DS) and calculated AUDPC values, as well as typical symptoms on infected wheat ears (appeared as lesions and discolouration) are presented in Fig. 3.4. Estimated disease severity was highest upon F. graminearum infection, followed by mixed infection (MIX A, MIX B), F. avenaceum and F. poae infection.

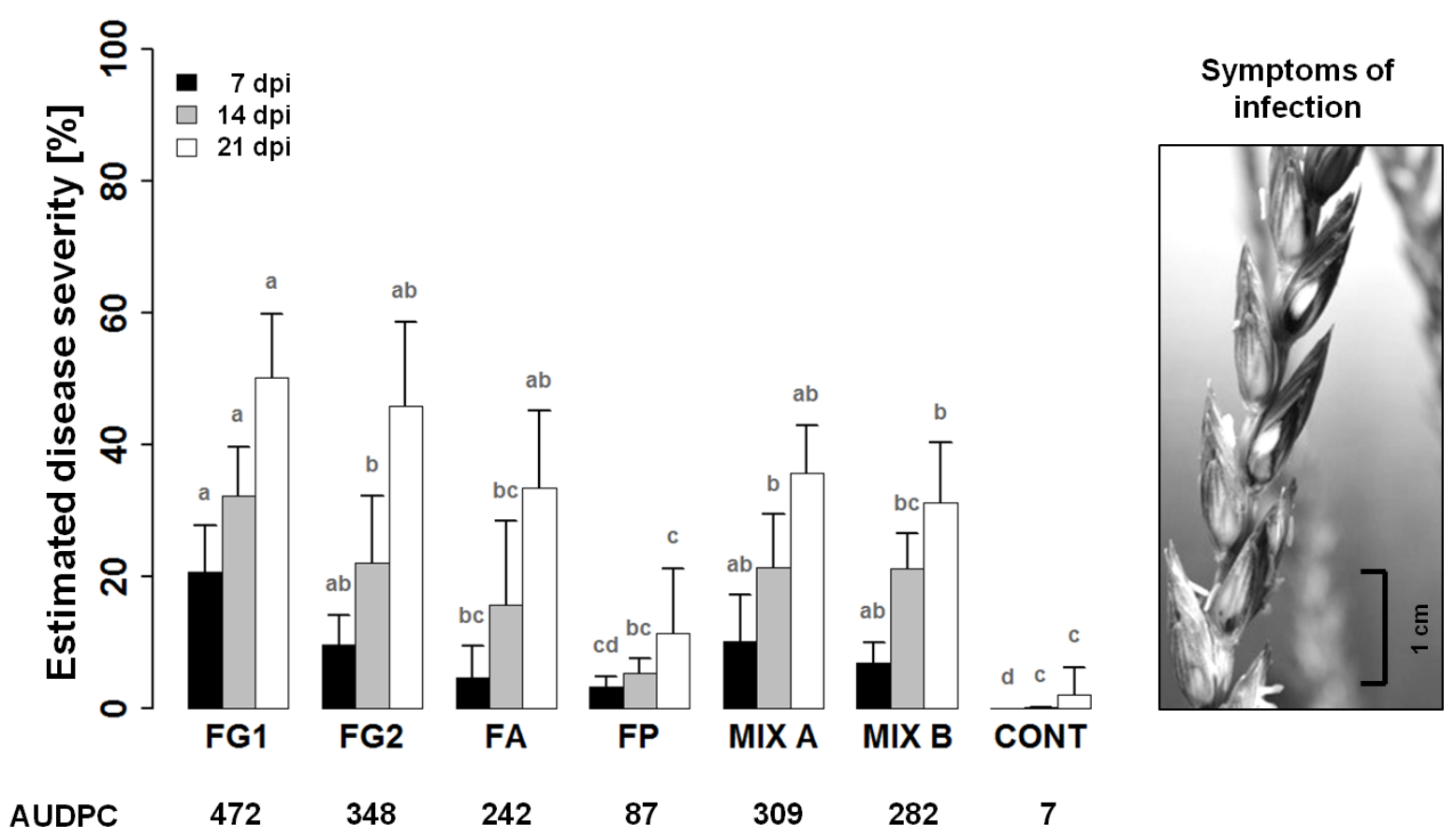

Fig. 3.4 Monitoring of disease progress on Fusarium infected wheat ears, estimated disease severity [\%] as mean \pm standard deviation within treatments (abbreviations of treatments FG1, FG2, FA, FP, MIX A, MIX B and CONT are described in Tab. 3.2); within plots different letters (a-d) represent statistical differences between treatments within time points $(7,14,21$ days postinoculation, dpi) (Scheffé test, $\mathrm{p}<0.05$ ); values for area under disease progress curve (AUDPC) were calculated according to the equation given in section 3.2.3, $7<n<8$; picture shows disease symptoms (as lesions and discoloration) after spray inoculation of the ear with Fusarium spores

\subsubsection{Mycotoxin Contamination and Fungal Biomass}

An overview of mycotoxin concentrations in Fusarium infected wheat at time point 21 dpi (end point) is given in Tab. 3.3. At this time point, DON was exclusively produced by $F$. graminearum strain FG2 (DON chemo type). NIV was produced by $F$. graminearum strain FG1 (NIV chemo type) and F. poae (FP). NIV appeared in both mixed infection treatments to lower extents than in FG1 single infection. Trichothecenes were not detected in control and $F$. avenaceum treated samples (even though trichothecenes appeared in every treatment with 
F. graminearum spores, their volatile precursor trichodiene was not detected by gas chromatographical analysis).

ENN B and ENN B1 appeared in both treatments with $F$. avenaceum spores (FA, MIX A), although concentration in single infection was 10 - 12 fold higher than in mixed treatment with FG1 (see Fig. 3.5). ENN A1 was only present in F. avenaceum single treatment. ENN B1 concentration was found to be 9 - 10 fold lower than ENN B. Furthermore, the concentrations of ENN B1 and ENN B were highly correlated $\left(\mathrm{R}^{2}=0.99\right)$. Beauvericin $(\mathrm{BEA})$ was detected in both treatments with $F$. poae spores (FP, MIX B).

Although BEA production was slightly reduced in mixed infection treatment, there was no suppression of $F$. poae biomass upon co-incubation with $F$. graminearum. The concentration of $F$. avenaceum biomass was 3 times lower in mixed infection treatment, compared to single infection, whereas F. graminearum biomass stayed constant (Fig. 3.5).

Tab. 3.3 Effect of infection treatment on the level of mycotoxins in wheat ears at time point 21 days post-inoculation (dpi)

\begin{tabular}{|c|c|c|c|c|c|c|c|}
\hline \multirow{2}{*}{ Infection treatment } & \multicolumn{7}{|c|}{ Toxin concentration in wheat flour [ $\mathrm{mg} \mathrm{kg}^{-1} \mathrm{DM} \pm{\text { standard deviation }]^{1}}^{1}$} \\
\hline & $\mathrm{DON}^{2}$ & $\mathrm{NIV}^{3}$ & ENN A ${ }^{4}$ & ENN & $\mathrm{NB}^{5}$ & ENN B1 ${ }^{6}$ & $\mathrm{BEA}^{7}$ \\
\hline Control & - & - & - & r & - & - & - \\
\hline F. avenaceum (FA) & - & - & $0.4 \pm 0.2$ & $24.7=$ & \pm 13.1 & $2.9 \pm 1.9$ & - \\
\hline F. graminearum (FG1) & - & $421.1 \pm 195.1$ & - & r & - & - & - \\
\hline F. graminearum (FG2) & $156.6 \pm 81.8$ & - & - & 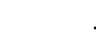 & - & - & - \\
\hline F. poae (FP) & - & $39.2 \pm 11.2$ & - & r & - & - & $0.81 \pm 0.73$ \\
\hline $\mathrm{MIX} A(\mathrm{FG} 1+\mathrm{FA})$ & - & $238.7 \pm 109.8$ & - & $2.1=$ & \pm 1.9 & $0.2 \pm 0.2$ & - \\
\hline $\mathrm{MIX} B(\mathrm{FG} 1+\mathrm{FP})$ & - & $193.3 \pm 62.9$ & - & . & - & - & $0.17 \pm 0.24$ \\
\hline \multicolumn{3}{|c|}{${ }^{1} \mathrm{DM}=$ dry matter, analysis by HPLC-MS, $\mathrm{n}=5$} & \multicolumn{5}{|c|}{${ }^{5}$ Enniatin B (ENN B), LOQ $=0.001 \mathrm{mg} \mathrm{kg}-1 \mathrm{DM}$} \\
\hline \multicolumn{3}{|c|}{2 Deoxynivalenol (DON), LOQ $=1 \mathrm{mg} \mathrm{kg}^{-1} \mathrm{DM}$} & \multicolumn{5}{|c|}{${ }^{6}$ Enniatin B1 (ENN B1), LOQ = $0.001 \mathrm{mg} \mathrm{kg}-1 \mathrm{DM}$} \\
\hline \multicolumn{3}{|c|}{${ }^{3}$ Nivalenol (NIV), LOQ $=1 \mathrm{mg} \mathrm{kg}^{-1} \mathrm{DM}$} & \multicolumn{5}{|c|}{${ }^{7}$ Beauvericin (BEA), $\mathrm{LOQ}=0.001 \mathrm{mg} \mathrm{kg}-1 \mathrm{DM}$} \\
\hline \multicolumn{3}{|c|}{${ }^{4}$ Enniatin A1 (ENN A1), LOQ $=0.001 \mathrm{mg} \mathrm{kg}^{-1} \mathrm{DM}$} & \multicolumn{5}{|c|}{$L O Q=$ Limit of quantification } \\
\hline
\end{tabular}

Time series revealed a constant accumulation of mycotoxins NIV and ENN B over time. The quantitative results are presented in Tab. 3.4 and illustrated in context with fungal biomass and DS over time in Fig. 3.6. The correlation between mean mycotoxin concentration and mean fungal biomass ( $F$. graminearum biomass and NIV concentration: $\mathrm{R}^{2}=1.00$, F. avenaceum biomass and ENN B concentration: $\mathrm{R}^{2}=0.68$ ) as well as between mean 
mycotoxin concentration and disease score over time (DS \% and NIV: $\mathrm{R}^{2}=1.00$, DS $\%$ and ENN B: $\mathrm{R}^{2}=0.99$ ) were high.



Fig. 3.5 Nivalenol (NIV) production and DNA concentration of $F$. graminearum (strain FG1) were not affected by mixed infection of summer wheat ears with $F$. avenaceum (FA) (MIX A is mixed infection treatment with FG1 and FA), while production of enniatins (shown for ENN B) and DNA concentration of $F A$ are significantly reduced in mixed infection compared to single infection treatment with FA only; statistical differences within treatments (t-test, $p<0.05)$ are labeled with letters $(a, b)$; time point of investigation $=21$ days after inoculation; $D M=$ dry matter, $Q 1=$ lower quartile (25\%), Q2 = median, Q3 = upper quartile (75\%); $n=5$

Tab. 3.4 Accumulation of nivalenol (NIV) and enniatins (ENN B/B1) in Fusarium infected wheat ears over 3 time points (7, 14 and 21 days post-inoculation, dpi)

\begin{tabular}{|c|c|c|c|c|c|c|c|c|c|}
\hline \multirow{4}{*}{$\begin{array}{l}\text { Time point } \\
7 \mathrm{dpi}\end{array}$} & \multicolumn{9}{|c|}{ Toxin concentration in wheat flour [ $\mathrm{mg} \mathrm{kg}^{-1} \mathrm{DM} \pm$ standard deviation $]^{\mathrm{a}}$} \\
\hline & \multirow{2}{*}{\multicolumn{3}{|c|}{$\begin{array}{c}\text { F. graminearum } \\
\text { NIV }\end{array}$}} & \multicolumn{6}{|c|}{ F. avenaceum } \\
\hline & & & & \multicolumn{3}{|c|}{ ENN B } & \multicolumn{3}{|c|}{ ENN B1 } \\
\hline & 286.4 & \pm & 18 & 7.5 & \pm & 1.4 & 0.9 & \pm & 0.2 \\
\hline 14 dpi & 419.5 & \pm & 113.5 & 24.9 & \pm & 15.6 & 3.3 & \pm & 2.3 \\
\hline $21 \mathrm{dpi}$ & 517 & \pm & 59.4 & 34.6 & \pm & 4.1 & 4.4 & \pm & 0.7 \\
\hline
\end{tabular}

${ }^{a}$ Mycotoxin concentration in dry flour of wheat ears infected with F. graminearum (FG1) or F. avenaceum (FA), analysis by HPLC-MS, DM = dry matter, limits of quantification (LOQ) are described in Tab. $3.3, n=3$ 
F. graminearum
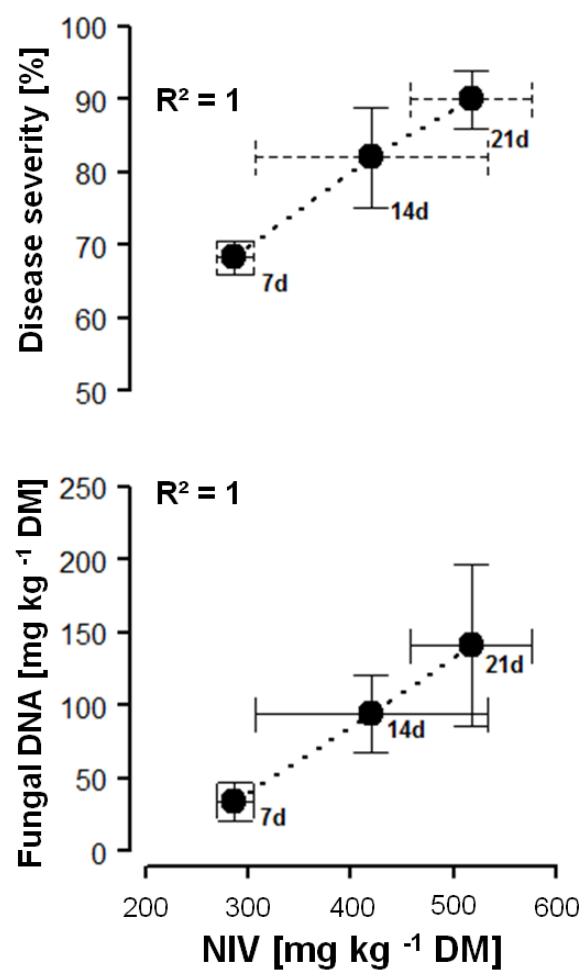

F. avenaceum
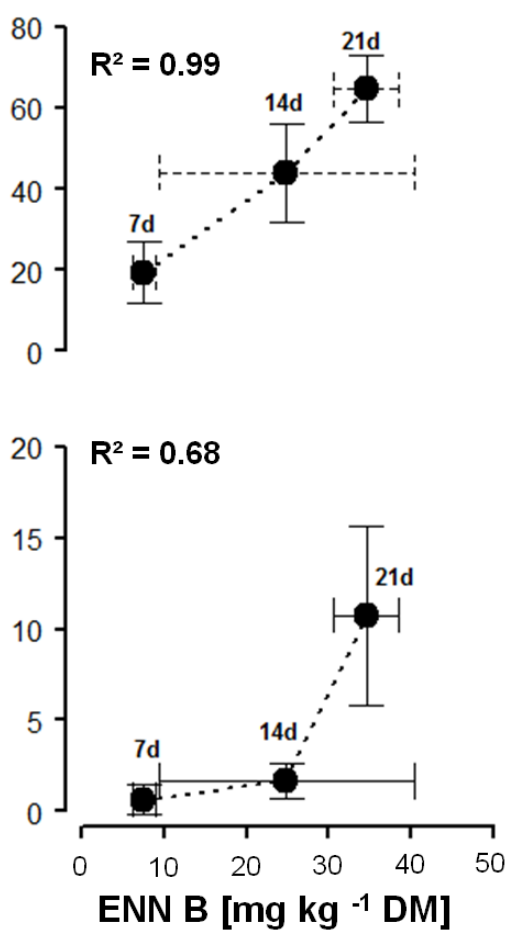

Fig. 3.6 Relation between level of mycotoxins (nivalenol (NIV), enniatin B (ENN B)), fungal DNA ( $F$. graminearum strain FG1, $F$. avenaceum strain FA) and estimated disease severity (DS \%) over three time points from 7 to 21 days after inoculation (d); data presented as mean values \pm standard deviation; correlations were calculated for means per time point; $\mathrm{DM}=$ dry matter; $\mathrm{n}=3$ 


\subsection{Discussion}

In the present study we identified a set of 13 volatile markers by SPME/GC-MS for the infection of summer wheat ears with toxigenic Fusarium species of different climatic allocations (F.graminearum, F. poae, F. avenaceum) (see Fig. 3.1 and Tab. 3.2). The biomarkers were detected from 7 dpi on, which was the first time point of our investigations.

Considering methodology, the suitability of the applied HS-SPME sampling for quantitative comparisons, with respect to the reproducibility of extraction, was examined and already discussed for maize (see chapter 2, section 2.2.9 for methodology and 2.3.2 for results). We assume that observed differences in the volatile profiles between both summer wheat trials and the fact that the profile of the second experiment was much more discriminative than the volatile pattern of the first one (see Fig. 3.1) were based on the different intensity of infection, which was stronger in the second trial. The different levels of disease severities might be caused by seasonal variations that affected greenhouse conditions (i.e. light conditions and temperature).

The volatile markers, released by Fusarium infected wheat ears, included common VOCs that have been reported in fungi and plants, such as (E)-2-hexenal (plant volatile) and 3-octanone (fungal origin). The group of those five to eight carbon containing molecules $\left(\mathrm{C}_{5}-\mathrm{C}_{8}\right)$ was largely coincident with the compounds identified in maize and was already discussed in chapter 2 .

In comparison to the previously reported results in maize, the chromatograms from Fusarium infected summer wheat did not reveal a large spectrum of terpenoid compounds. Other authors as well reported only a small group of terpenes, such as ubiquitous $\beta$-caryophyllene, from wheat attacked by fungal pathogens or insects (Piesik et al., 2011; Jeleń et al., 1995). Only one volatile terpene, trichodiene, was detected by Perkowski et al. (2008) in infected wheat under field conditions. The set of volatile biomarkers, described in the present work (see Tab. 3.2, Fig. 3.1) included only two compounds of terpenoid origin (sesquiterpenes, KI 1421, KI 1467). These compounds could not be identified by mass spectral libraries. Nevertheless, both volatiles were not present in non-infected wheat ears and serve, therefore, as qualitative markers for Fusarium infection (i.e. present or absent). Not statistically confirmed as marker compounds, but of particular interest because of the general assumption 
that terpenoids show a certain specifity to ecological interactions (Pichersky \& Gershenzon, 2002), is the group of other volatile terpenoids that were detected specifically in the case of F. avenaceum (monoterpenes, KI 962, KI 976) and F. poae (sesquiterpenes, KI 1541, KI 1578) infection. The identification by mass spectral libraries was not possible for these terpenes either. In contrast to other observations, including our own results in maize, obtained with the same sampling procedure and adsorbing material, we could not identify volatile trichodiene from Fusarium infected wheat tissue. This was surprising, because we observed the formation of trichothecenes (i.e. DON, NIV) to a large extent. One reason might be that the volatile precursor is efficiently converted to non-volatile products and is, therefore, not released in detectable concentrations.

In genreal, the described selection of 13 volatile markers allows to distinguish between 'infected' and 'non-infected' wheat, but offers few possibilities to categorize between different Fusarium species. In this context, it has to be considered that under natural conditions it is more likely that FHB is caused by a multiple species complex, than by a single species (Waalwijk et al., 2003). In opposite to the described results in maize, we found mainly quantitative (i.e. 2-heptanone, compound KI 919) and few qualitative differences (sesquiterpenes, unknown compound KI 1516) between treatments. Especially F. avenaceum and $F$. graminearum infected plants show quite similar volatile patterns. F. poae infection, revealing lowest disease severities (Fig. 3.4) among all tested Fusarium species, barely shared signals of the other treatments (neither of healthy plants nor of F. graminearum and F. avenaceum infection). Similarly to maize (chapter 2), it has to be checked, whether the present set of biomarkers could be transferred to the field (i.e. for a screening for volatile markers with portable detectors) and serve as fast decision guidance for plant protection and food safety strategies.

Apart from the VOC biomarkers, we monitored disease severities, mycotoxin concentrations and fungal DNA. As expected, these parameters were constantly increasing from 7 dpi to 21 dpi and were highly correlated (as observed for F. graminearum and $F$. avenaceum). It has to be considered that the mycotoxin concentrations in infected ears, especially DON and NIV, were abnormally high. Generally, is not expected to find such high concentrations under natural conditions in the field (Bottalico \& Perrone, 2002; Placinta et al., 1999). Interestingly, we found a significant suppression of $F$. avenaceum DNA and enniatin production upon mixed infection with $F$. graminearum (MIX A), whereas the latter species stayed 
unchallenged in the mixed treatment (Fig. 3.5). A suppression of $F$. avenaceum by $F$. graminearum was not reported yet.

\section{Acknowledgements}

We acknowledge all partners within the project MykoSensExpert (2010 - 2012), which was funded by the German Ministry of Education and Research and supported by German Areospace centre (DLR). Furthermore, we acknowledge KWS Saat AG who supplied the wheat seeds and Dr. Antonio Prodi (University of Bologna, Italy) who kindly provided the $F$. poae strain.

\section{References}

Anonymus (2007) Commission regulation (EC) no. 1126/2007amending Regulation (EC) No $1881 / 2006$ setting maximum levels for certain contaminants in foodstuffs as regards Fusarium toxins in maize and maize products. Official Journal of the European Union 255:14-17.

Bai G-H, Shaner G (1996) Variation in Fusarium graminearum and Cultivar Resistance to Wheat Scab. Plant Disease 80:975-979.

Bennett JW, Klich M (2003) Mycotoxins. Clinical Microbiology Reviews 16:497-516.

Boerjesson T, Stoellman U, Adamek P, Kaspersson A (1989) Analysis of volatile compounds for detection of molds in stored cereals. Cereal Chemistry 66:300-304.

Bottalico A, Perrone G (2002) Toxigenic Fusarium species and mycotoxins associated with head blight in small-grain cereals in Europe. European Journal of Plant Pathology 108:611-624.

Brandfass C, Karlovsky P (2008) Upscaled CTABbased DNA extraction and real-time PCR assays for Fusarium culmorum and F. graminearum DNA in plant material with reduced sampling error. International Journal of Molecular Sciences 9:23062321.

Campagnoli A, Cheli F, Polidori C, et al. (2011) Use of the Electronic Nose as a Screening Tool for the Recognition of Durum Wheat Naturally Contaminated by Deoxynivalenol: A Preliminary Approach. Sensors 11:4899-4916.
D’Mello JPF, Placinta CM, Macdonald AMC (1999) Fusarium mycotoxins: a review of global implications for animal health, welfare and productivity. Animal Feed Science and Technology 80:183-205.

Demyttenaere JC., Moriña RM, De Kimpe N, Sandra P (2004) Use of headspace solidphase microextraction and headspace sorptive extraction for the detection of the volatile metabolites produced by toxigenic Fusarium species. Journal of Chromatography A 1027:147-154.

Doell S, Daenicke S (2011) The Fusarium toxins deoxynivalenol (DON) and zearalenone (ZON) in animal feeding. Preventive Veterinary Medicine 102:132-145.

Eifler J, Martinelli E, Santonico M, et al. (2011) Differential detection of potentially hazardous Fusarium species in wheat grains by an electronic nose. PLoS ONE 6:e21026.

Girotti JR, Malbrán I, Lori GA, Juárez MP (2010) Use of solid phase microextraction coupled to capillary gas chromatographymass spectrometry for screening Fusarium spp. based on their volatile sesquiterpenes. World Mycotoxin Journal 3:121-128.

Girotti JR, Malbrán I, Lori GA, Juárez MP (2012) Early detection of toxigenic Fusarium graminearum in wheat. World Mycotoxin Journal 5:143-152. 
Goswami RS, Kistler HC (2004) Heading for disaster: Fusarium graminearum on cereal crops. Molecular Plant Pathology 5:515525 .

Jeleń HH, Latus-Ziętkiewicz D, Wąsowicz E, Kamiński E (1997) Trichodiene as a volatile marker for trichothecenes biosynthesis. Journal of microbiological methods 31:45-49.

Jeleń HH, Mirocha CJ, Wąsowicz E, Kamiński E (1995) Production of volatile sesquiterpenes by Fusarium sambucinum strains with different abilities to synthesize trichothecenes. Applied and Environmental Microbiology 61:38153820 .

Krska R, Schubert-Ullrich P, Molinelli A, et al. (2008) Mycotoxin analysis: An update. Food Additives \& Contaminants: Part A 25:152-163.

Kuiper-Goodman T, Scott PM, Watanabe H (1987) Risk assessment of the mycotoxin zearalenone. Regulatory Toxicology and Pharmacology 7:253-306.

McMullen M, Jones R, Gallenberg D (1997) Scab of Wheat and Barley: A Re-emerging Disease of Devastating Impact. Plant Disease 81:1340-1348.

Miedaner T, Moldovan M, Ittu M (2003) Comparison of spray and point inoculation to assess resistance to Fusarium head blight in a multienvironment wheat trial. Phytopathology 93:1068-1072.

Miller JD (2008) Mycotoxins in small grains and maize: Old problems, new challenges. Food Additives \& Contaminants: Part A 25:219-230.

Morgavi DP, Riley RT (2007) An historical overview of field disease outbreaks known or suspected to be caused by consumption of feeds contaminated with Fusarium toxins. Animal Feed Science and Technology 137:201-212.

Niessen L (2007) PCR-based diagnosis and quantification of mycotoxin producing fungi. International Journal of Food Microbiology 119:38-46.

Parry DW, Jenkinson P, McLEOD L (1995) Fusarium ear blight (scab) in small grain cereals - a review. Plant Pathology 44:207-238.
Perkowski J, Buśko M, Chmielewski J, et al. (2008) Content of trichodiene and analysis of fungal volatiles (electronic nose) in wheat and triticale grain naturally infected and inoculated with Fusarium culmorum. International journal of food microbiology 126:127-134.

Pichersky E, Gershenzon J (2002) The formation and function of plant volatiles: perfumes for pollinator attraction and defense. Current Opinion in Plant Biology 5:237243.

Piesik D, Panka D, Delaney KJ, et al. (2011) Cereal crop volatile organic compound induction after mechanical injury, beetle herbivory (Oulema spp.), or fungal infection (Fusarium spp.). Journal of Plant Physiology 168:878-886.

Placinta C, D’Mello JP, Macdonald AM (1999) A review of worldwide contamination of cereal grains and animal feed with Fusarium mycotoxins. Animal Feed Science and Technology 78:21-37.

Presicce DS, Forleo A, Taurino AM, et al. (2006) Response evaluation of an E-nose towards contaminated wheat by Fusarium poae fungi. Sensors and Actuators B: Chemical 118:433-438.

Schnuerer J, Olsson J, Boerjesson T (1999) Fungal volatiles as indicators of food and feeds spoilage. Fungal Genetics and Biology 27:209-217.

Simko I, Piepho H-P (2012) The Area Under the Disease Progress Stairs: Calculation, Advantage, and Application. Phytopathology 102:381-389.

Tholl D, Boland W, Hansel A, et al. (2006) Practical approaches to plant volatile analysis. The Plant Journal 45:540-560.

Waalwijk C, Kastelein P, De Vries I, et al. (2003) Major changes in Fusarium spp. in wheat in the Netherlands. European Journal of Plant Pathology 109:743-754.

Zadoks JC, Chang TT, Konzak CF (1974) A decimal code for the growth stages of cereals. Weed Research 14:415-421.

Zinedine A, Soriano JM, Moltó JC, Mañes J (2007) Review on the toxicity, occurrence, metabolism, detoxification, regulations and intake of zearalenone: An oestrogenic mycotoxin. Food and Chemical Toxicology 45:1-18. 


\title{
4 A Bioassay for Zearalenone (ZEN) Based on the Mycoparasite Gliocladium roseum
}

\begin{abstract}
Fusarium spp. cause severe problems in the production of maize (Zea mays L.), wheat (Triticum aestivum L.) and other small-grain cereals worldwide. The infection causes yield losses and contamination of food and feedstuff with toxic secondary metabolites, so called mycotoxins. Among this group of metabolites, the macrocyclic acid lactone zearalenone (ZEN). The toxin exerts estrogenic effects in mammals and humans. It is produced by several Fusarium spp. including F. graminearum, F. culmorum, F. equiseti and F. crookwellense. Until now, several bioassays have been described for an inexpensive detection of ZEN in agricultural commodities. Those bioassays are mainly based on the human estrogen receptors $\alpha$ and $\beta$ and do not specifically respond to ZEN or its derivates.

The soil borne mycoparasite Gliocladium roseum Bainier (syn. Clonostachys rosea) has been extensively studied as a biocontrol agent against fungal diseases of plants. G. roseum is able to detoxify ZEN by secretion of a specific lactonase. The goal of the present study was to develop an inexpensive and specific high-throughput bioassay, based on a recombinant G. roseum strain that carries a fusion of the ZEN-sensing promotor-element (zes2) with a reporter gene (green fluorescent protein, GFP), for the detection of ZEN and its reductive metabolites in complex matrices, such as maize flour.

Maize plants were grown in the field and infected with ZEN-producing Fusarium spp. Harvested ears were dried and ground. Aliquots were extracted and cleaned with different procedures including conventional solid-phase extraction and immunoaffinity clean-up (IAC). The G. roseum indicator strain was grown in 96-well microplates. Fluorescence of GFP was measured with a fluorescence reader (FLX800, Biotek) in time series after addition of ZEN extracts. Apart from the quantitative approach, the use of the bioassay for a qualitative high throughput fungal strain screening for ZEN production was evaluated.
\end{abstract}


We found an approximately linear relation between ZEN concentrations in maize samples predicted by the devoloped G. roseum ZEN-bioassay and the determined concentrations by HPLC-MS after IAC clean-up (ZEN concentrations ranged from $0.9 \mathrm{mg} \mathrm{kg}^{-1}$ to $90 \mathrm{mg} \mathrm{kg}^{-1}$ ). The assay is therefore suitable for complex matrices, such as maize. In a screening of fungal strains, a precise division of strains into ZEN- producers and non-producers was possible.

The presented approach could serve as pioneering work to use sensitive biological systems, such as antagonistic interactions of microorganisms, for the detection of biological active metabolites. 


\subsection{Introduction}

Filamentous fungi of the genus Fusarium cause severe problems in the production of smallgrain cereals and maize (Zea mays L.) worldwide (Logrieco et al., 2002; McMullen et al., 1997). The infection, which is usually caused by a multiple Fusarium species complex, leads to typical symptoms described as 'Fusarium head blight' (FHB) of wheat (Triticum aestivum L.) and other small-grain cereals and 'red ear rot' or 'pink ear rot' of maize. Economical losses are a consequence of the reduction in yield and product quality, due to contamination with toxic metabolites of fungi, named mycotoxins (Miller, 2008; Bennett \& Klich, 2003; Tanaka et al., 1988). Most important Fusarium mycotoxins, as described in D'Mello et al. (1999), are trichothecenes, fumonisins and zearalenone (ZEN).

ZEN (previously known as F-2 toxin) is a macrocyclic $\beta$-resorcylic acid lactone containing an olefinic double bond and keto group [6-(10-hydroxy-6-oxo-trans-1-undecenyil)-resorcylic acid lactone]. The structure was first described by Urry et al. (1966) (see Fig. 4.1). The nonsteroidal compound is produced in the polyketide pathway of numerous Fusarium spp. including F. graminearum Schwabe, F. culmorum (W. G. Smith), F. equiseti (Corda) Sacc. sensu Gordon and F. crookwellense Burgess (Bennett \& Klich, 2003; Bottalico \& Perrone, 2002; Caldwell et al., 1970). The benefit of ZEN for phytopathogenic Fusarium spp. is still not fully understood, but Utermark \& Karlovsky (2007) described its inhibitory effect on the growth of filamentous fungi and emphasized its advantageous role in competition.

ZEN is commonly found in feed and foodstuff in temperate regions worldwide. Its synthesis usually takes place before harvest, but inappropriate storage conditions, such as high humidity, may also lead to an accumulation (Zinedine et al., 2007; Kuiper-Goodman et al., 1987). With a melting point between $161-163{ }^{\circ} \mathrm{C}$, ZEN is comparatively stable and withstands most processing steps in food production (Jackson \& Bullerman, 1999).

ZEN, which is hardly toxic $\left(\mathrm{LD}_{50}\right.$ (female rat) $\left.>10,000 \mathrm{mg} \mathrm{kg}^{-1}\right)$, binds to estrogen receptors and resembles 17ß-estradiol (Zinedine et al., 2007; Kuiper et al., 1998). Because of its powerful estrogenic effect on mammals and humans it is considered to be a nonsteroidal estrogen or mycoestrogen, rather than a mycotoxin (Bennett \& Klich, 2003). Especially in pigs, which are highly susceptible, but also other animals, ZEN may lead to hyperestrogenism, disrupted conception, abortion as well as suppression of the innate immunity (Marin et al., 2010; 
Kuiper-Goodman et al., 1987). Furthermore, it has been reported that ZEN leads to hyperestrogenic syndroms and precocious pubertal development in woman (Massart \& Saggese, 2010). Other studies described a carcinogenic (Yu et al., 2005; Ahamed et al., 2001) and genotoxic (Stopper et al., 2005; Lioi et al., 2004; Pfohl-Leszkowicz et al., 1995) effect of ZEN.

The stereoisomers $\alpha$-zearalenol ( $\alpha$-ZOL) and $\beta$-zearalenol $(\beta$-ZOL) are reductive metabolites of ZEN. $\alpha-Z O L$ is considered to be several times more estrogenic (Gromadzka et al, 2008; Hagler et al., 1979). Both isomers are also known to have cytotoxic effects in mammals, such as the induction of oxidative damage and apoptosis (Othmen et al., 2008). A semi-synthetic anabolic agent, based on the ZEN-related compound $\alpha$-zearalanol (zeranol) (Ralone ${ }^{\circledR}$, Ralgro ${ }^{\circledR}$ etc.) has been prohibited in the European Union since 1981, but is still in use in beef production in other countries. Even its illegal use as a sports drug in humans was reported (Thevis et al., 2011). The zeranol metabolites $\beta$-zearalanol (taleranol) and zearalanone were also reported to possess estrogenic properties (Leffers et al., 2001). Fig. 4.1 illustrates the structures of ZEN, its reductive metabolites $\alpha-Z O L$ and $\beta-Z O L$ and the human estrogen $17 \beta$-estradiol.

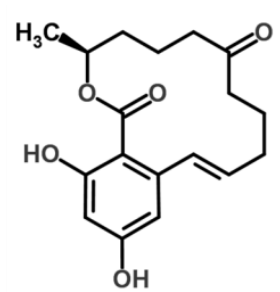

zearalenone<smiles>C[C@@H](O)CCC[C@H](O)CC/C=C/c1cc(O)cc(O)c1C(=O)O</smiles>

a-zearalenol



$\beta$-estradiol

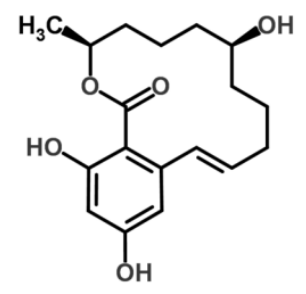

$\beta$-zearalenol
Fig. 4.1 Chemical structures of estrogenic zearalenone (ZEN), 17ß-estradiol and stereoisomers of zearalenol ( $\alpha$-zearalenol ( $\alpha$-ZOL), $\beta$-zearalenol ( $\beta$-ZOL))

The provisional maximum tolerable daily intake (PMTDI) of ZEN was documented to be 0.5 $\mu \mathrm{g} \mathrm{kg}^{-1}$ body weight (Zinedine et al., 2007). Because of the apparent health risks, the European Commission established maximum levels for ZEN in foodstuff (Commission Regulation (EC) no. 1126/2007). Hence, the maximum ZEN level in bread and other bakery products is determined to be $50 \mu \mathrm{g} \mathrm{kg}^{-1}$. 
The analysis of ZEN and its derivates is usually performed with liquid chromatography, combined with fluorescence detection (Tanaka et al., 1985) or mass spectrometry (Songsermsakul et al., 2006). Also enzyme-linked immunosorbent assays (ELISAs) for ZEN are still in use (Bennett et al., 1994). A clean-up of contaminated sample material (i.e. corn, foodstuff) using immunoaffinity columns (IAC) prior to chromatographical analysis, is considered as a suitable method for the reduction of undesired matrix effects (Erbs et al., 2007; Visconti \& Pascale, 1998).

Because the analysis by liquid chromatography is expensive and time-consuming, researchers attempted to develop a cheap bioassay for the detection of ZEN and other estrogenic compounds in foodstuff. Until now, several bioassays mainly based on the human estrogen receptors $\alpha$ and $\beta$ have been described (Winter et al., 2008; Bovee et al., 2004; Mitterbauer et al., 2003). These bioassays respond to physiological estrogens as well as to phyto- and mycoestrogens. Thus, they can not be used for a specific detection of ZEN and its derivates.

The mycoparasite Gliocladium roseum Bainier (syn. Clonostachys rosea) is a common biocontrol agent against phytopathogenic fungi, such as Botrytis cinerea, the causal agent of grey mould in fruits and vegetables (Morandi et al., 2000; Sutton et al., 1997). Beside other protective enzymes, G. roseum secretes a specific lactonase that converts ZEN to nonestrogenic cleavage products (el-Sharkawy \& Abul-Hajj, 1988).

The aim of the present study was to develop a sensitive and inexpensive high-throughput bioassay, based on a G. roseum zes2::gfp strain that carries a fusion of the ZEN sensing promoter element (zes2) with a reporter gene (green fluorescent protein, GFP), for the specific detection of ZEN and its most important metabolite $\alpha-Z O L$ in complex matrices, such as corn flour. 


\subsection{Materials and Methods}

\subsubsection{Recombinant Gliocladium roseum Strain and Spore Production}

A recombinant $G$. roseum strain (wild type $(w t)=$ DSM 62726 , obtained from Leibniz Institute DSMZ, Braunschweig, Germany), carrying a fusion of the zearalenone-sensing promotor (zes2) with a reporter gene (GFP, S65T, Acc. No. EF090408) was constructed by Dr. J. Utermark (Molecular Phytopathology and Mycotoxin Research, University of Goettingen, Germany). The process of Agrobacterium tumefaciens-mediated transformation is described in Utermark \& Karlovsky (2006). An activation of the ZEN-sensing promoter leads to fluorescence emission by the constructed zes $2:: g f p$ strain. The recombinant G. roseum strain and wt are listed in Tab. 4.1.

G. roseum spores were produced in a liquid mung bean medium, following a modified protocol of Bai \& Shaner (1996). For this purpose, $80 \mathrm{~g}$ of mung beans were transferred to $1 \mathrm{~L}$ of demineralized water. The water was boiled until all beans opened and, subsequently, filtered through cotton wool. Portions of $250 \mathrm{~mL}$ were distributed to Erlenmeyer flasks and autoclaved $\left(121^{\circ} \mathrm{C}, 20 \mathrm{~min}\right)$. Aliquots of $250 \mu \mathrm{L}$ G. roseum spore suspension or, respectively, grown mycelium on agarose plugs $(0.5 \mathrm{~cm} \times 0.5 \mathrm{~cm})$, were transferred to the prepared flasks. Cultures were incubated for 15 days at $25^{\circ} \mathrm{C}$ and dark conditions on a rotary shaker. Afterwards, suspensions were filtered through cotton wool and centrifuged at $2440 \mathrm{~g}$ for $10 \mathrm{~min}$. Supernatant was discarded and spores were re-dissolved in $500 \mu \mathrm{L}$ glycerol $(15 \%$ (v/v), Carl Roth, Karlsruhe, Germany). Suspensions were stored at $-70{ }^{\circ} \mathrm{C}$ and germination tests were performed frequently on potato dextrose agar (PDA, Carl Roth, Karlsruhe, Germany) to assess the viability of spores.

\subsubsection{Fungal Material for Stain Screening}

A set of 38 fungal strains, mainly Fusarium spp. (ZEN-producers and non-producers), were cultivated for a fungal strain screening (described in sections 4.2.13-4.2.16). An overview of fungal material is presented in Tab. 4.1. 
Tab. 4.1 Overview of fungal material

\begin{tabular}{|c|c|c|c|}
\hline Fungal species & Isolate No./ Code & Origin & Source \\
\hline \multirow[t]{3}{*}{ Fusarium avenaceum } & DSM 62161 & Dianthus caryophyllus, Germany & $A$ \\
\hline & CBS 121.73 & Dianthus caryophyllus, England & $\mathrm{B}$ \\
\hline & DSM 21724 & Beta vulgaris, Germany & $A$ \\
\hline F. culmorum & Fc 4.0 & unknown & $\mathrm{C}$ \\
\hline \multirow[t]{2}{*}{ F. equiseti } & O90 & unknown & $\mathrm{D}$ \\
\hline & $\mathrm{O} 20$ & unknown & $\mathrm{D}$ \\
\hline \multirow[t]{13}{*}{ F. graminearum } & FG210.1 & unknown & $\mathrm{C}$ \\
\hline & FG71 & unknown & $E$ \\
\hline & $102.3 \mathrm{wt}$ & unknown & $\mathrm{C}$ \\
\hline & FG 7-34 & Triticum aestivum, Australia & $E$ \\
\hline & FG 201.1 wt & unknown & C \\
\hline & $\mathrm{FG} 108.1 \mathrm{wt}$ & unknown & $\mathrm{C}$ \\
\hline & FG 104.2 wt & unknown & C \\
\hline & $\mathrm{FG} 203.1 \mathrm{wt}$ & unknown & $\mathrm{C}$ \\
\hline & $\mathrm{FG} 210$ & unknown & C \\
\hline & FG 106.2 wt & unknown & $\mathrm{C}$ \\
\hline & $\mathrm{FG} 211.1 \mathrm{wt}$ & unknown & $\mathrm{C}$ \\
\hline & $\mathrm{Fg} \mathrm{7-28} \mathrm{wt}$ & unknown & $E$ \\
\hline & $\mathrm{Fg} 7-29 \mathrm{wt}$ & unknown & $E$ \\
\hline \multirow[t]{2}{*}{ F. oxysporum } & DSM 2018 & Hordeum vulgare, Germany & $A$ \\
\hline & DSM 62291 & Citrus sinensis, Germany & $A$ \\
\hline \multirow[t]{2}{*}{ F. poae } & FS 41 & unknown & $\mathrm{F}$ \\
\hline & F448 & unknown & $\mathrm{F}$ \\
\hline \multirow[t]{4}{*}{ F. proliferatum } & DSM 764 & unknown & $A$ \\
\hline & DSM 62267 & Zea mays, Iran & $A$ \\
\hline & DSM 62261 & Cymbidium hybrid, Germany & A \\
\hline & DSM 63267 & Sansevieria trifasciata, Germany & $A$ \\
\hline \multirow[t]{2}{*}{ F. subglutinans } & CBS 215.76 & Zea mays, Germany & $\mathrm{B}$ \\
\hline & PK 2-17-7 & Zea mays, China & $\mathrm{G}$ \\
\hline \multirow[t]{2}{*}{ F. tricinctum } & $\mathrm{O} 63$ & unknown & $\mathrm{D}$ \\
\hline & O32 & unknown & D \\
\hline \multirow[t]{5}{*}{ F. verticillioides } & Fv lta 1 & unknown & $\mathrm{H}$ \\
\hline & FRC M-8114 & USA & 1 \\
\hline & DSM 62264 & Zea mays, Germany & $A$ \\
\hline & GF A & Zea mays, USA & J \\
\hline & GF F & Sorghum bicolor, USA & $\mathrm{J}$ \\
\hline \multirow[t]{2}{*}{ Gliocladium roseum } & wt (DSM 62726) & Kalanchoe sp., wilting plant, Germany & $A$ \\
\hline & zes2::gfp & recombinant strain of wt (DSM 62726) & C \\
\hline \multirow[t]{2}{*}{ Trichoderma harzianum } & T.s. & unknown & K \\
\hline & $\mathrm{T} 12$ & unknown & $\mathrm{L}$ \\
\hline
\end{tabular}

$\mathbf{A}=$ Leibniz Institute - DSMZ, Braunschweig, Germany, B = Fungal Biodiversity Centre, Utrecht, The Netherlands , C = Fungal strain collection, Plant Pathology, University of Goettingen, Germany, D = Institute for Sugar Beet Research, Goettingen, Germany, E = T. Miedaner, University of Hohenheim, Germany, $\mathbf{F}=$ A. Prodi, University of Bologna, Italy, $\mathbf{G}=$ P. Karlovsky, University of Goettingen, Germany, $\mathbf{H}=$ F. Cardinale, University of Turin, Italy, I = FRC Pennsylvania, USA, J = B. Tudzynski, University of Muenster, Germany, K = Sauter \& Stepper GmbH, Ammerbuch, Germany, $\mathbf{L}=$ University of Hannover, Germany 


\subsubsection{Plant Material from Field Trials}

Several cultivars of commercial hybrid maize were grown under field conditions. Maize ears were inoculated with spore suspensions of different $F$. graminearum strains at main flowering stage, as described by Reid et al. (1995). Maize ears were harvested at late maturing stage, dehusked and oven-dried. Afterwards, material was ground and homogenized. A representative set of samples was selected, based on quantitative real-time PCR analysis for $F$. graminearum (data not shown). Wheat material from field trails (partially inoculated with Fusarium spp.) was collected from different regions in Germany. The ear material was oven-dried, ground and homogenized as well. Similarly to maize, the selection of wheat samples followed species-specific PCR analysis (data not shown).

\subsubsection{General Setup of the G. roseum Bioassay and Measurement Procedure}

All experiments were carried out in liquid GM7 medium as published by Utermark \& Karlovsky (2007). Cultures of the G. roseum zes $2: \because g f p$ strain were grown in sterile flat-bottom 96-well microplates with lid (transparent, Sarstedt, Nuembrecht, Germany). For this purpose, $46 \mu \mathrm{L}$ liquid GM7 medium and $8 \mu \mathrm{L}$ spore suspension, with an adjusted spore concentration of 675000 spores $\mathrm{mL}^{-1}$, were transferred to each well. The microplates were centrifuged at $1627 \mathrm{~g}$ for $60 \mathrm{~s}$ to collect the suspension at the bottom and transferred to an autoclaved glass container with lid (further described as growing chamber, $20 \mathrm{~cm}$ diameter, $5 \mathrm{~cm}$ height). The microplate lid was opened to facilitate gas exchange. For maintenance of humidity in the growing chamber a Petri dish (60 mm diameter, Sarstedt, Nuembrecht, Germany) was filled with $20 \mathrm{~mL}$ sterile water and added to each container. The glass container was sealed with one layer of Parafilm ${ }^{\circledR} \mathrm{M}$ (American National Can, Greenwich, CT, USA). Cultures were incubated at $25^{\circ} \mathrm{C}$ in darkness. After $48 \mathrm{~h}$ of pre-growing, $6 \mu \mathrm{L}$ of ZEN analyte in methanol-water (MW) (40:60, v/v) were added to each well with a G. roseum zes2::gfp culture. Methanol content within $60 \mu \mathrm{L}$ total reaction volume per well was below $5 \%(\mathrm{v} / \mathrm{v})$ and, therefore, not considered as harmful for the fungus. Cultures were incubated again at $25{ }^{\circ} \mathrm{C}$ in darkness.

First fluorescence measurement (read) was carried out $24 \mathrm{~h}$ after addition of analyte. Further measurement steps followed in intervals of $12 \mathrm{~h}$. Cultures were covered during transport to protect the light sensitive assay. A Biotek FLx800 fluorescence microplate reader (Biotek, 
Bad Friedrichshall, Germany) was used to detect fluorescence emission, expressed as relative fluorescence unit (RFU), of the G. roseum zes $2:: g f p$ strain in exposition to ZEN. The machine was equipped with an optical filter set for excitation at $485 \pm 20 \mathrm{~nm}$ and emission at $528 \pm 20$ $\mathrm{nm}$. Temperature during measurements was set to $25^{\circ} \mathrm{C}$. The parameter of optical sensitivity, with respect to signal-to-noise ratio, was adjusted according to the manufacturers' instructions prior to the first measurements. Bottom readings were carried out to achieve the smallest distance between fluorescence sensor and growing culture. Blank readings of empty wells (bare plastic) were carried out to subtract the background of the plate. Analysis was performed with Gen5 ${ }^{\mathrm{TM}}$ microplate data collection \& analysis software (Biotek, Bad Friedrichshall, Germany).

The appearance of propagules and mycelium of G. roseum as well as the incubation chamber and the instrument for fluorescence detection are illustrated in Fig. 4.2. Furthermore, the general setup procedure with $48 \mathrm{~h}$ pre-culturing of the G. roseum zes $2:: g f p$ indicator strain, is illustrated in Fig. 4.3 A.

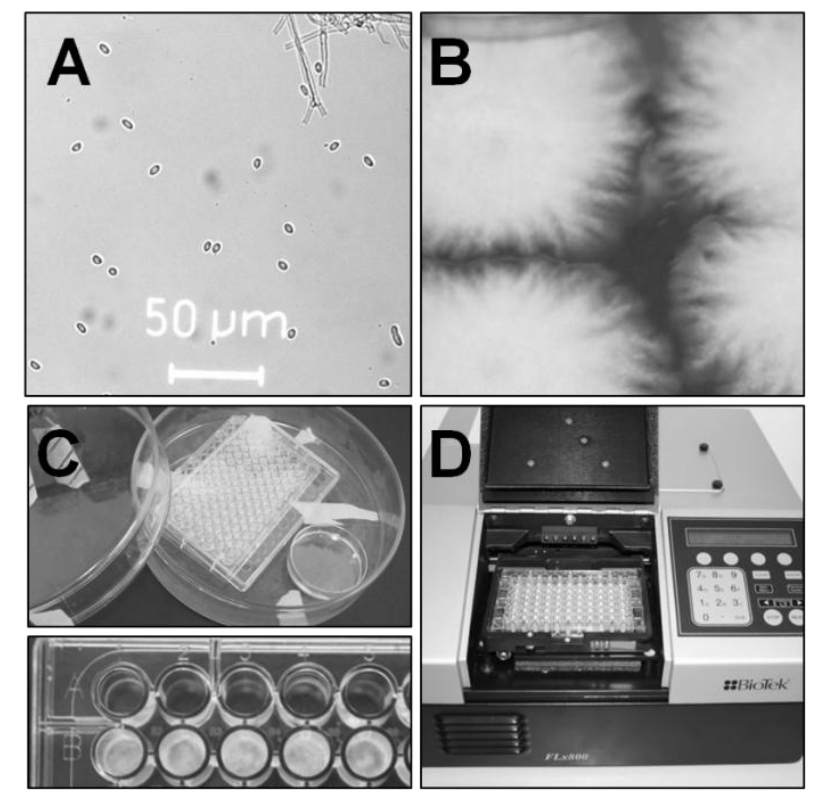

Fig. 4.2 Morphological traits of G. roseum and images of the bioassay for zearalenone (ZEN), (A) conidia of G. roseum, (B) growing culture of the G. roseum zes2::gfp strain on GM7 agarose medium, (C) glass incubator with microplate and growing cultures in wells (picture below), (D) fluorescence reader with microplate

\subsubsection{Assessment of Bioassay Kinetics and Detection Limits in General Procedure}

A ZEN standard row in concentrations of $1.0-27.5 \mu \mathrm{g} \mathrm{mL}^{-1}$ was prepared in MW (40:60, v/v, LC-MS grade, Th. Geyer, Renningen, Germany, double distilled water). The used ZEN Stock (10 $000 \mu \mathrm{g} \mathrm{ZEN} \mathrm{mL}{ }^{-1}$ ) was obtained from Fermentek (Jerusalem, Israel). The prepared standard row was transferred in 6 replications to cultures of the G. roseum zes $2:: g f p$ strain as 
described for the general setup procedure (section 4.2.4) (final concentrations in medium were in the range of $\left.0.1-2.75 \mu \mathrm{g} \mathrm{mL}^{-1}\right)$. Pure MW (40:60, v/v) was added in 12 replications as negative control. Fluorescence measurements were carried out at 24, 36 and $48 \mathrm{~h}$ after addition of the analyte. The time point of maximum response of G. roseum zes2::gfp strain towards ZEN, expressed as RFU, was selected as best time point for quantitative purposes. The limit of detection (LOD) was calculated as mean RFU of the negative control (zes2::gfp strain treated with MW only) plus 3 times standard deviation and the limit of quantification (LOQ) was determined as mean RFU of the negative control plus 9 times standard deviation.

\subsubsection{Evaluation of Assay Specifity to ZEN, its Reductive Metabolites and Other Estrogens}

$\beta$-Estradiol $(\beta$-EST) $(\geq 98 \%$, as powder, Sigma-Aldrich, Taufkirchen, Germany) was dissolved in pure methanol and, subsequently, diluted to concentrations of 5, 15 and $20 \mu \mathrm{g} \mathrm{mL}^{-1}$ in MW (40:60, v/v). Stereoisomers $\alpha$-zearalenol $(\alpha-Z O L)$ and $\beta$-zearalenol ( $\beta$-ZOL) (as powder, both obtained from Sigma-Aldrich, Taufkirchen, Germany) were dissolved in acetonitrile $(\mathrm{ACN})$ and diluted to concentrations of 10,20 and $30 \mu \mathrm{g} \mathrm{mL}^{-1}$ in MW (40:60, v/v). Samples were added in two independent replications to G. roseum zes $2:: g f p$ culture as described earlier (general setup, section 4.2.4). Final concentrations in medium were, therefore, $0.5,1.0,1.5$ and $2 \mu \mathrm{g} \beta$-EST ml ${ }^{-1}$ and 1.0, 2.0 and $3.0 \mu \mathrm{g} \alpha$-ZOL / $\beta-\mathrm{ZOL}$

$\mathrm{mL}^{-1}$. Furthermore, the fluorescence emission of $G$. roseum wt in exposition to ZEN was assessed. Pure ZEN standards in concentrations of 1.0, 2.0 and $3.0 \mu \mathrm{g} \mathrm{mL}^{-1}$ (in medium) were used as reference for a positive response. Fluorescence readings were carried after 24, 36 and $48 \mathrm{~h}$ as described earlier (section 4.2.4).

\subsubsection{Alternative Setup - A Procedure with Direct Exposure of G. roseum Spores to ZEN}

Aliquots of $60 \mu \mathrm{L}$ pure ZEN standards in concentrations between 0.05 and $4.0 \mu \mathrm{g} Z \mathrm{ZEN} \mathrm{mL}^{-1}$ in MW (80:20, v/v, 10 concentration steps) were transferred in 6 replications to a 96-well microplate. Pure MW (80:20, v/v) was added in 12 replications as negative control. The filled microplates were transferred to a glass bowl with lid (equipped with spacer to facilitate air exchange) and the organic solvent was evaporated under a fume hood. Thereafter, a volume of $52 \mu \mathrm{L}$ liquid GM7 medium was transferred to the dry residue. After 60 min of resting at 
room temperature, $8 \mu \mathrm{L}$ of a G. roseum zes $2: \because g f p$ spore suspension (675000 spores $\mathrm{mL}^{-1}$ ) were added to each well and plates were centrifuged for $60 \mathrm{~s}$ at $1627 \mathrm{~g}$. Cultures were incubated in a glass bowl at $25{ }^{\circ} \mathrm{C}$ in darkness. Measurement of fluorescence response started after $24 \mathrm{~h}$ and continued until $60 \mathrm{~h}$. The process of this alternative procedure, also described as direct exposure procedure, is illustrated in Fig. 4.3 B.

A)
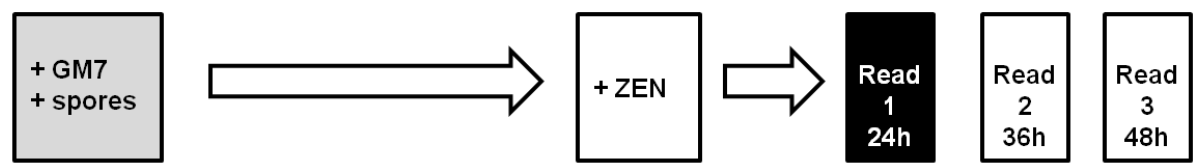

B)
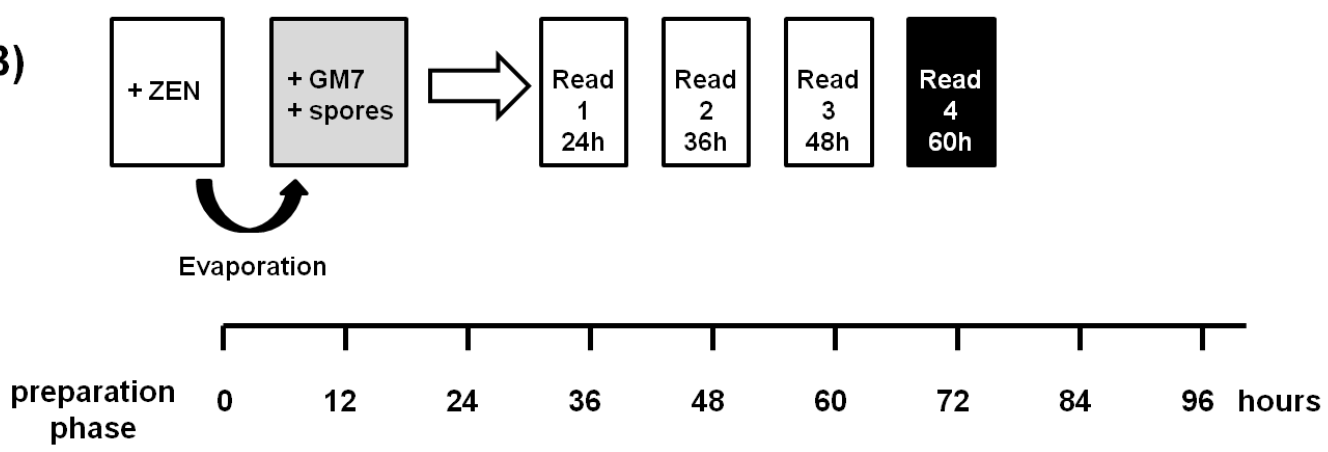

Fig. 4.3 Time schedule of assessed procedures for G. roseum zes2::gfp bioassay for zearalenone (ZEN) detection, (A) general procedure: fungal cultures were incubated for $48 \mathrm{~h}$ before ZEN containing analyte was added (section 4.2.4), (B) alternative procedure: spores of G. roseum zes2::gfp were immediately exposed to ZEN (section 4.2.7); favoured time points for fluorescence readings are labelled in black (selection of optimal time points for measurements are described in detail in results section)

\subsubsection{General Procedure with Solvent Extracts of Maize Field Samples After Clean-up with Immunoaffinity Columns (IAC)}

ZEN was extracted from contaminated maize material including a clean-up step with IAC (ZearaStar $^{\circledR}$, Romer Labs, Tulln, Austria, ZEN-capacity of $2000 \mathrm{ng}$ per column). At first, $10 \mathrm{~g}$ of homogenized maize flour were transferred to a $50 \mathrm{~mL}$ centrifuge tube (Sarstedt, Nuembrecht, Germany) and blended with $40 \mathrm{~mL}$ ACN-water (75:25, v/v, ACN was purchased from Th. Geyer, Renningen, Germany). Samples were homogenized and transferred to a shaker for one hour. After this, samples were centrifuged for $10 \mathrm{~min}$ at $7320 \mathrm{~g}$. To reduce the content of organic solvent, as required by IAC manufacturer, $4 \mathrm{~mL}$ of each supernatant were transferred to a new centrifuge tube and diluted with $36 \mathrm{~mL}$ PBS-buffer (phosphate buffered saline, $137 \mathrm{nM} \mathrm{NaCl}$ (Carl Roth, Karlsruhe, Germany), $2.7 \mathrm{mM} \mathrm{KCl}, 12 \mathrm{mM}$ total phosphate ( $\mathrm{HPO}_{4}{ }^{2-}$ and $\mathrm{H}_{2} \mathrm{PO}_{4}^{-}, \mathrm{Na}_{2} \mathrm{HPO}_{4}, \mathrm{KH}_{2} \mathrm{PO}_{4}$ ), $\mathrm{pH} 7.4$ was adjusted with $\mathrm{HCL}$, all chemicals, except $\mathrm{NaCl}$, were purchased from Merck, Darmstadt, Germany). ZEN concentrations in 
samples from artificial inoculated field trails were expected to be very high, but the capacity of employed IAC was limited to $2 \mu \mathrm{g}$. Thus, the dilution in PBS-buffer was repeated twice to obtain 3 dilution steps (1:10 (v/v), 1:100 (v/v) and 1:1000 (v/v)). Further clean-up was carried out according to the IAC-manufacturers' instructions. Samples were eluted from the IAC matrix with $1.5 \mathrm{~mL}$ methanol and subsequently evaporated to dryness in a vacuum concentrator $\left(35^{\circ} \mathrm{C}\right.$, overnight, Christ, Osterode, Germany). Dry residues were re-dissolved in $35 \mu \mathrm{L} \quad \mathrm{MW}$ (40:60, v/v). Reference measurements with high-performance liquid chromatography mass spectrometry (HPLC-MS, 1200 LC equipped with Mistral 510 column oven (Varian, Darmstadt, Germany) and Kinetex ${ }^{\circledR}$ C18 column (Phenomex Inc., Aschaffenburg, Germany)), were carried out with a 1:100 (v/v) dilution of each sample extract in MW (50:50, v/v).

For the purpose of quantification, dilution series from sample extracts were prepared to ensure the comparability of RFU values of unknown samples to the RFU values of the applied ZEN standard row. The final extracts were diluted 3 times in 1:2 (v/v) steps with MW (40:60, v/v). Hence, 4 subsamples were generated $\left(\mathrm{V} 0_{\mathrm{IAC}}=\right.$ undiluted extract, $\mathrm{V} 1_{\mathrm{IAC}}=1: 2$ dilution, $\mathrm{V} 2_{\mathrm{IAC}}=1: 4$ dilution, $\mathrm{V} 3_{\mathrm{IAC}}=1: 8$ dilution). Prior to use, extracts were stored at $-20{ }^{\circ} \mathrm{C}$. Each sample was transferred in two replications to G. roseum zes $2: \because g f p$ culture plates as described for the general setup (section 4.2.4). The process of sample preparation including IAC cleanup is illustrated in Fig. 4.4.

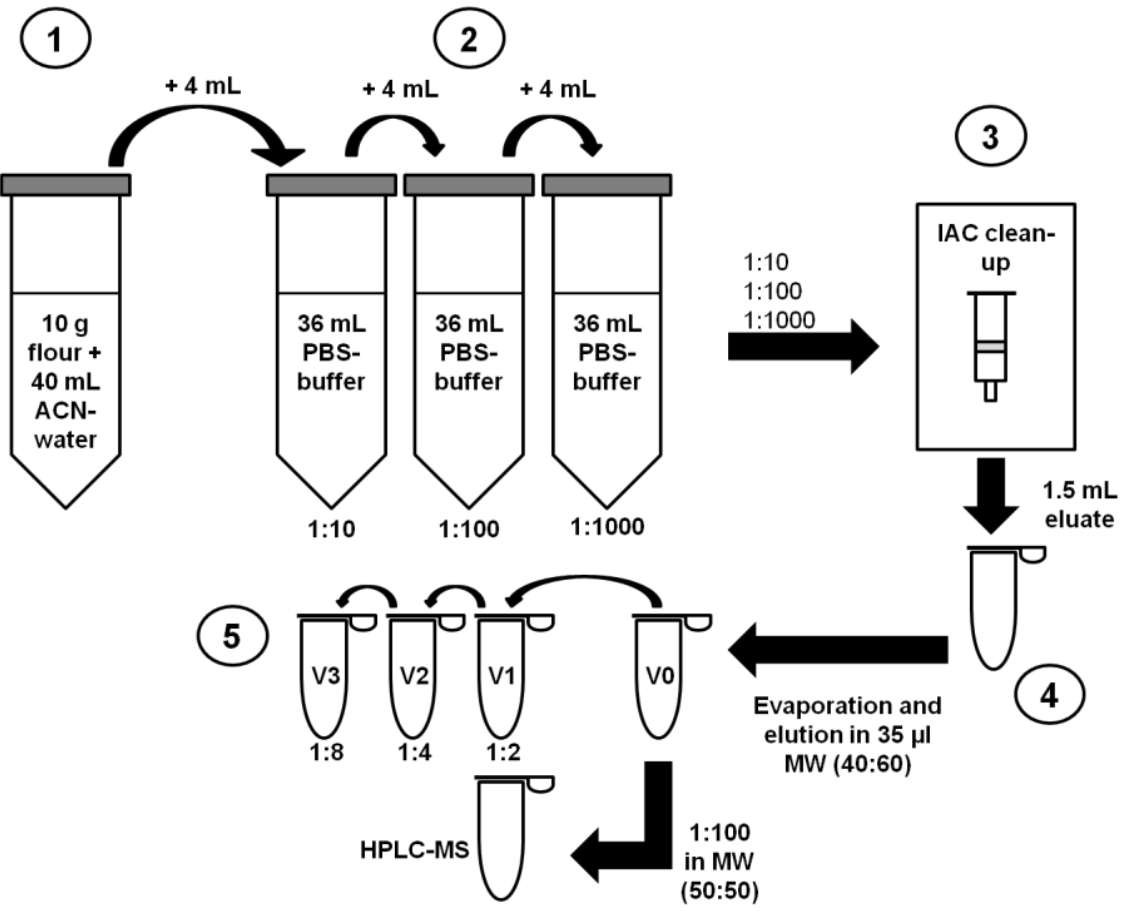

Fig. 4.4 Sample preparation from maize flour with immunoaffinity columns (IAC), preparation followed the order from step 1 to 5 , Abbreviations: $\mathrm{ACN}=$ acetonitrile, PBS = phosphate buffered saline, $\mathrm{MW}=$ methanolwater (a ratio of 40:60 $(\mathrm{v} / \mathrm{v})$ was used for the sample preparation for G.roseum zes2::gfp assay, a ratio of 50:50 $(\mathrm{v} / \mathrm{v})$ was used for the preparation of HPLC-MS reference samples 


\subsubsection{General Procedure with Extracts of Maize Field Samples After Conventional Solid-Phase Extraction (SPE)}

Conventional solid-phase extraction (SPE) with Bond Elut Mycotoxin columns (BE, Agilent, Waldbronn, Germany) was carried out as a second clean-up procedure of solvent extracts from maize field samples. At first, $2 \mathrm{~g}$ of homogenized maize flour were blended with $18 \mathrm{~mL}$ ACN-water (84:16, v/v). Samples were shaken and centrifuged as described for IAC clean-up (section 4.2.8). Cyclohexane (Carl Roth, Karlsruhe, Germany) was used for sample defatting. Afterwards, $4 \mathrm{~mL}$ of the lower phase were transferred to BE columns (see Fig. $4.12 \mathrm{~A}$ and B). The eluate was captured in $15 \mathrm{~mL}$ centrifuge tubes. Aliquots of $2.8 \mathrm{~mL}$ were transferred to new tubes and evaporated to dryness in a vacuum concentrator $\left(35^{\circ} \mathrm{C}\right.$, overnight $)$. The residues were re-dissolved in $40 \mu \mathrm{L}$ MW (40:60, v/v). Similarly to IAC clean-up, the final extracts were diluted in 1:2 (v/v) steps with $\mathrm{MW}(40: 60, \mathrm{v} / \mathrm{v})\left(\mathrm{V}_{\mathrm{BE}}=\right.$ undiluted sample, $\mathrm{V} 1_{\mathrm{BE}}=1: 2$ dilution, $\mathrm{V} 2_{\mathrm{BE}}=1: 4$ dilution).

ZEN extracts from maize material were added to the pre-cultured G. roseum zes $2: \because g f p$ strain as described for the general setup (section 4.2.4). Fluorescence readings were carried out at time points 24,36 and $48 \mathrm{~h}$ after addition of the analyte.

\subsubsection{Calculation of Matrix Correction Factors for IAC and Conventional SPE Clean- up of Maize Samples in General Procedure}

Non-contaminated maize material from field trials (confirmed as not contaminated by real-time PCR) was extracted and cleaned with both IAC and BE columns as described earlier (sections 4.2.8 and 4.2.9), including the 3 additional dilutions steps with PBS-buffer in IAC procedure. Prepared extracts were spiked with ZEN in concentrations between 0.0 and $40 \mu \mathrm{g} \mathrm{ml}^{-1}$. Each Spiked sample was transferred in 3 replications to cultures of the G. roseum zes2::gfp strain as described for the general setup (section 4.2.4). The matrix effect of these samples equals the effect of undiluted sample extracts (earlier described as V0 dilution). Pure ZEN standards in MW (40:60, v/v, same concentration range) were used as a reference. Fluorescence readings were carried out at time points 24,36 and $48 \mathrm{~h}$ after addition of analyte.

Matrix effects, or respectively, factors of matrix correction (cf, see formula below) were determined to enable the calculation of ZEN concentrations in field samples on the basis of 
ZEN standard rows in MW. For this purpose, the factors between mean RFU values of each ZEN concentration step in matrix and pure MW were calculated. Afterwards, the factors were averaged within a defined matrix background (IAC or conventional SPE) at a certain time point after addition of analyte $(\mathrm{t}=24,36$ or $48 \mathrm{~h})$. It was assumed that the calculated matrix effects for V0 (undiluted) were reduced by $50 \%$ with each 1:2 dilution step of the field samples (applied dilution steps IAC: $\mathrm{V} 0_{\text {IAC }}, \mathrm{V} 1_{\text {IAC }}, \mathrm{V} 2_{\text {IAC }}, V 3_{\text {IAC }}$; dilution steps for conventional SPE (BE): $\left.\mathrm{V} 0_{\mathrm{BE}}, \mathrm{V} 1_{\mathrm{BE}}, \mathrm{V} 2_{\mathrm{BE}}\right)$.

$$
\begin{aligned}
& \text { Matrix correction: } \mathrm{X}_{1 t}=\mathrm{X}_{0 t} / \mathrm{cf} t \\
& \mathrm{X}_{1 t}=\text { matrix corrected RFU at time point } t \\
& \mathrm{X}_{0 t}=\mathrm{RFU} \text { (blank value) at time point } t \\
& c f_{t}=\text { factor of matrix correction at time point } t
\end{aligned}
$$

\subsubsection{Bioassay with Extracts from Field Samples Employing Conventional SPE Clean- up and Alternative Procedure}

Extraction of ZEN from field samples of maize $(n=12)$ and wheat $(n=8)$ was carried out with ACN-water (84:16, v/v) followed by clean-up with BE columns as described earlier (section 4.2.9, no concentration under vacuum). ZEN standard rows in maize and wheat matrix were constructed (ZEN concentrations between 0.5 and $4.0 \mu \mathrm{g} \mathrm{ml}^{-1}$ ). HPLC-MS measurements of unknowns were carried out as reference. Aliquots of $60 \mu 1$ (sample extracts and standards) were added to the microplates and further procedure followed the instructions as given in section 4.2.7.

\subsubsection{Data Processing and Calculation of ZEN Concentration in Field Samples}

ZEN concentrations in field samples were calculated on the basis of ZEN standard rows in matrix (no correction) or MW (with matrix correction). For the quantification of ZEN in maize field samples after clean-up with conventional SPE or IAC (general setup with $48 \mathrm{~h}$ pre-growing of the fungus), the blank RFU values (raw data) were first corrected for matrix effects as given in the equation (see equation for matrix correction, 4.2.10). After this, ZEN concentrations were calculated according to the standard row in MW. Based on these data, the quantification of ZEN in maize flour was performed in consideration of the extraction process (i.e. volumes, dilutions, aliquots). The suitability of the G. roseum 
$z e s 2:: g f p$ assay for quantification of ZEN in complex matrices was evaluated on the basis of HPLC-MS reference measurements.

\subsubsection{Cultivation of Fungal Strains and Extraction of ZEN for Strain Screening}

Fungal strains were cultivated on rice medium. For this purpose, $3 \mathrm{~g}$ rice kernels (BAK Kardeşler $\mathrm{GmbH}$, confirmed as not contaminated with mycotoxins by HPLC-MS analysis) were transferred to $50 \mathrm{~mL}$ centrifuge tubes. A volume of $2 \mathrm{~mL}$ demineralized water was added to the kernels. Tubes were closed with cotton stoppers and autoclaved $\left(121^{\circ} \mathrm{C}\right.$, $20 \mathrm{~min})$. Selected fungal strains were pre-cultivated. For this purpose, diluted spore suspensions were transferred to PDA medium and incubated at $25{ }^{\circ} \mathrm{C}$. After $48 \mathrm{~h}$, single colonies were collected and transferred to new medium. After 2 days, overgrown discs with young fungal mycelium were replaced with a cork borer $(0.5 \mathrm{~cm}$ diameter, one disc per fungal strain) and transferred to the prepared rice substrate. Fungal cultures were incubated for 6 weeks at $22{ }^{\circ} \mathrm{C}$ in darkness (see Fig. $4.12 \mathrm{C}$ and D). After incubation, cultures were blended with $30 \mathrm{~mL}$ ACN-water (84:16, v/v) and shaken over night. Next day, samples were centrifuged for $10 \mathrm{~min}$ at $7320 \mathrm{~g}$. For each sample, an aliquot of $6 \mathrm{~mL}$ was collected from the clear supernatant and transferred to a new centrifuge tube. The extracts were blended with $1 \mathrm{x}$ volume cyclohexane to remove fatty acids and centrifuged again. A volume of $4 \mathrm{~mL}$ from the lower phase was transferred to BE column. The flow-through of $2.8 \mathrm{ml}$ was collected in a new tube and evaporated to dryness. Dry residues were dissolved in $400 \mu \mathrm{L} \mathrm{MW} \mathrm{(80:20,} \mathrm{v/v).}$ Each sample was diluted 1:10 (v/v) with MW (80:20, v/v) and stored at $-20{ }^{\circ} \mathrm{C}$ prior to use. HPLC-MS analysis was carried out as reference.

\subsubsection{Plate Layout for Fungal Strain Screening}

Fungal strain screening for ZEN production was carried out as described earlier for the alternative setup. The process included direct exposure of G. roseum zes $2: \because g f p$ strain to extracts without pre-growing of the fungus (section 4.2.7). Experiments were carried out in two independent replications (two microplates, $24 \mathrm{~h}$ time shift between replications). For this purpose, $60 \mu \mathrm{L}$ of each sample extract (undiluted and 1:10 diluted) and 3 negative controls (MW only) were transferred to the wells. Solvent was evaporated and zes $2:: g f p$ spores with GM7 medium were supplemented as described earlier. Measurements of fluorescence emission were carried out after 24, 36 and $48 \mathrm{~h}$ of incubation. 


\subsubsection{Use of Defatted Crude Extracts from in vitro Cultures for Fungal Strain Screening}

A selection of 9 defatted crude extracts from fungal cultures on rice medium was collected prior to BE clean-up (section 4.2.13). The extracts were obtained from cultures of 3 F. graminearum strains (FG210.1, FG71, FG211.1 wt), 2 strains of $F$. verticillioides (GFA, FRC M-8114) and one strain of each F. culmorum (Fc 4.0), F. proliferatum (DSM 62261), F. tricinctum (O63) and Trichoderma harzianum (T12). Further procedure followed the description as given in section 4.2.14.

\subsubsection{Data Evaluation for Fungal Strain Screening}

Strains were assigned to be ZEN-producers if fluorescence signal exceeds a signal-to-noise ratio of 20. The signal of noise or, respectively, background was defined as mean fluorescence signal of the negative control (treated with MW only). 


\subsection{Results}

\subsubsection{Characterization of the Bioassay}

\subsubsection{Response of G. roseum zes2::gfp Strain to ZEN and Other Estrogens}

So far, the available estrogen bioassays do not respond specifically to mycoestrogens. Hence, the response of the G. roseum zes $2:: g f p$ fusion strain towards $\beta$-EST and the zearalenol stereoisomers $\alpha$-ZOL and $\beta$-ZOL was tested. The emission of fluorescence by $G$. roseum $z e s 2:: g f p$ strain was upregulated upon exposure with ZEN and $\alpha-\mathrm{ZOL}$, but not other estrogenic compounds. As expected, G. roseum wild type revealed no fluorescence emission exceeding background signal. The same was observed for G. roseum zes $2: \because g f p$ strain exposed to MW only (negative control). The Response of G. roseum zes $2: \because g f p$ strain to selected estrogenic molecules is illustrated in Fig. 4.5.



Fig. 4.5 Fold change in fluorescence emission by G. roseum zes2::gfp strain relative to negative control in cultures exposed to different concentrations of estrogenic $\beta$-estradiol ( $\beta$ EST), $\alpha$-zearalenol ( $\alpha$-ZOL), $\beta$-zearalenol ( $\beta$-ZOL) and zearalenone $(Z E N)$; applied procedure followed general setup as described in section 4.2.4; measurements were carried out at time point $24 \mathrm{~h}$ after addition of analyte; interrupted horizontal threshold line presents fluorescence emission of G. roseum zes2::gfp strain treated with pure methanol-water (40:60, v/v) (negative control, equals 1); presented is one out of two data sets (both replications are in accordance), data illustrate the specificity of the bioassay for $\alpha-Z O L$ and ZEN 


\subsubsection{Assay Kinetics in General Procedure with Pre-Culturing of G. roseum}

The temporal development of fluorescence emission by G. roseum zes $2: \because g f p$ strain, as response to broad range of ZEN concentrations, was examined and limits of detection were assessed. Maximum response of the pre-cultured G. roseum zes $2:: g f p$ strain towards ZEN was detected at an early time point of $24 \mathrm{~h}$ after addition of the estrogenic mycotoxin. This was observed for all assessed concentrations of ZEN (below $2.5 \mu \mathrm{g} \mathrm{ml}^{-1}$ ). Fluorescence emission by zes $2:: g f p$ strain dropped continuously from $24 \mathrm{~h}$ on. Measurements were also performed at earlier time points than $24 \mathrm{~h}$, but these earlier readings resulted in a lower response to ZEN and a high variation within concentration steps (data not shown). The time point of maximum response $(24 \mathrm{~h})$ was determined as optimal time point for quantitative purposes. Fig. 4.6 illustrates the temporal development of fluorescence emission for four selected concentration steps.
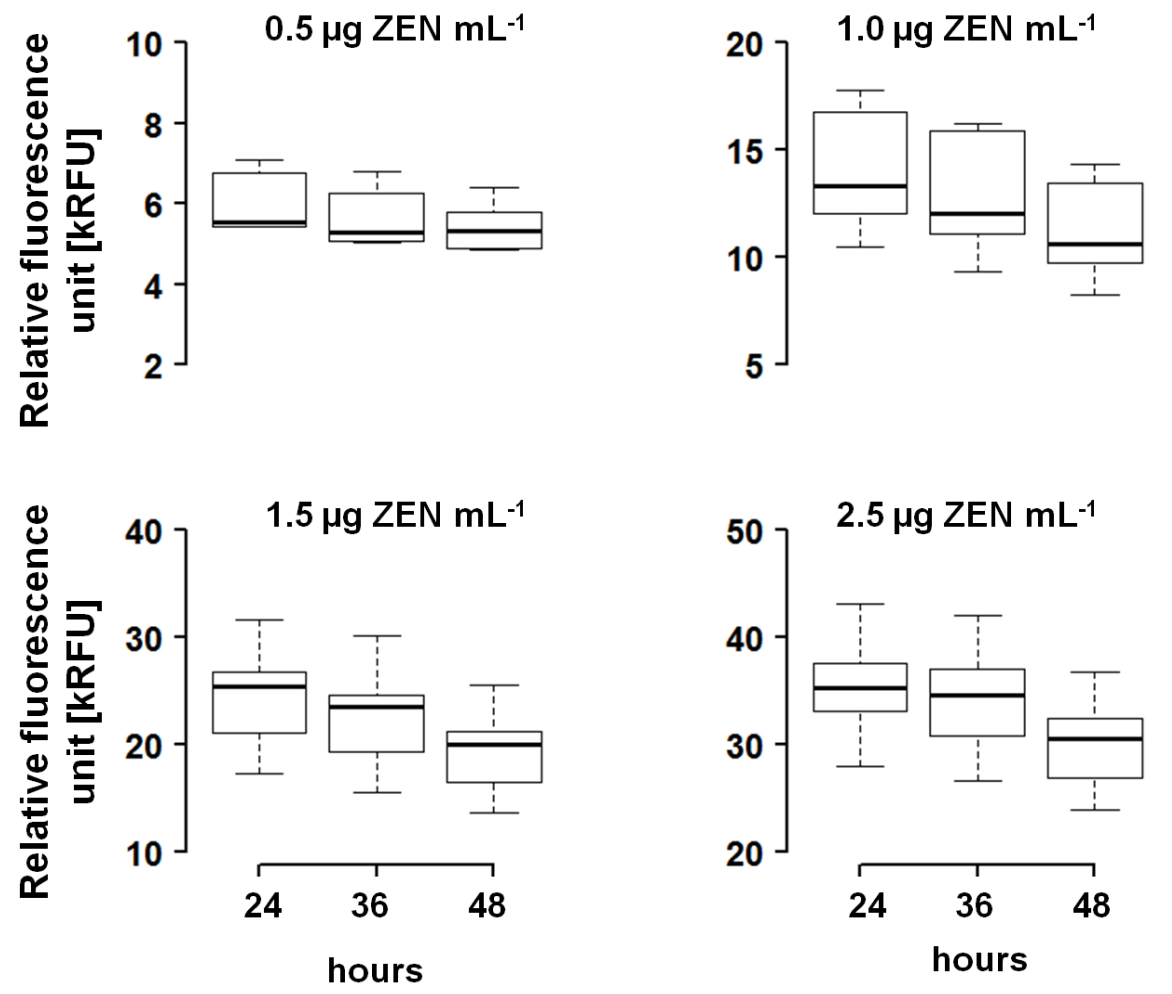

Fig. 4.6 Temporal response, expressed as fluorescence emission, of G. roseum zes2::gfp strain (general procedure, section 4.2.4) to zearalenone (ZEN) in concentrations of $0.5,1.0,1.5$, and $2.5 \mu \mathrm{g} \mathrm{mL}^{-1}$ in medium, revealed maximum response at 24 hours after addition of ZEN containing analyte, time point $24 \mathrm{~h}$ was favoured for further analysis, Q1= lower quartile (25\%), Q2 = median, Q3 = upper quartile (75\%), $\mathrm{n}=6$ 
The dose dependent response of G. roseum zes $2: \because g f p$ strain towards ZEN at the favoured time point of $24 \mathrm{~h}$ is illustrated in Fig. 4.7. Calculated limit of detection (LOD) was $0.05 \mu \mathrm{g} \mathrm{ZEN} \mathrm{ml}^{-1}$ in medium and limit of quantification (LOQ) was determined to be $0.13 \mu \mathrm{g} \mathrm{ZEN} \mathrm{ml} l^{-1}$ in medium.

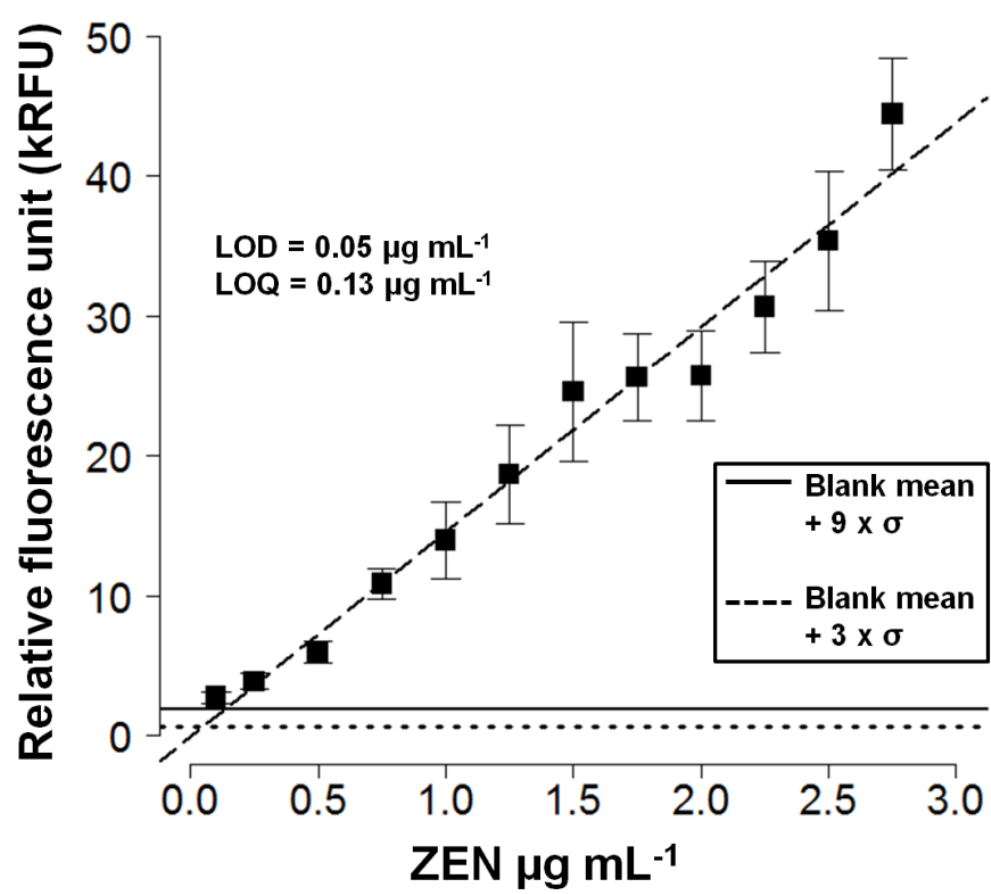

Fig. 4.7 Dose dependent response of pre-cultivated G. roseum zes2::gfp strain to zearalenone (ZEN) in medium, at time point $24 \mathrm{~h}$ after addition of ZEN analyte, revealed strong correlation $(r=0.99)$, handling of strain is described in general procedure (section 4.2.4), response is expressed as relative fluorescence unit (RFU) (mean \pm standard deviation (o')); calculated fluorescence thresholds for limit of detection (LOD, blank mean +3 times $\sigma$ ) and limit of quantification (LOQ, blank mean +9 times o') are illustrated as horizontal lines (see legend), LOD was $0.05 \mu \mathrm{g} Z E N \mathrm{~mL}^{-1}$ and LOQ was $0.13 \mu \mathrm{g} \mathrm{ZEN} \mathrm{mL}{ }^{-1}$, $\mathrm{n}=6$

The effect of maize matrix on the fluorescence response of pre-cultured G. roseum zes $2: \because g f p$ strain towards ZEN was assessed for the extraction with immunoaffinity columns (IAC) and conventional SPE clean-up (BE). The effects are illustrated in Fig. 4.8. The matrix effect was determined for 3 time points $(24 \mathrm{~h}, 36,48 \mathrm{~h})$ after addition of ZEN containing analyte. As described earlier, the extracts from plant material were diluted in several steps with PBSbuffer prior to IAC clean-up and, subsequently, concentrated under vacuum. No differences in matrix effect were found between these preparative dilution steps (see Fig. 4.8). Thus, the obtained matrix effects for IAC clean-up were combined. For the favoured time point of $24 \mathrm{~h}$ after addition of ZEN, the factor of matrix correction (cf) was determined to be 1.43 for IAC clean-up and 0.73 for conventional SPE clean-up. 
hours 24

36

48

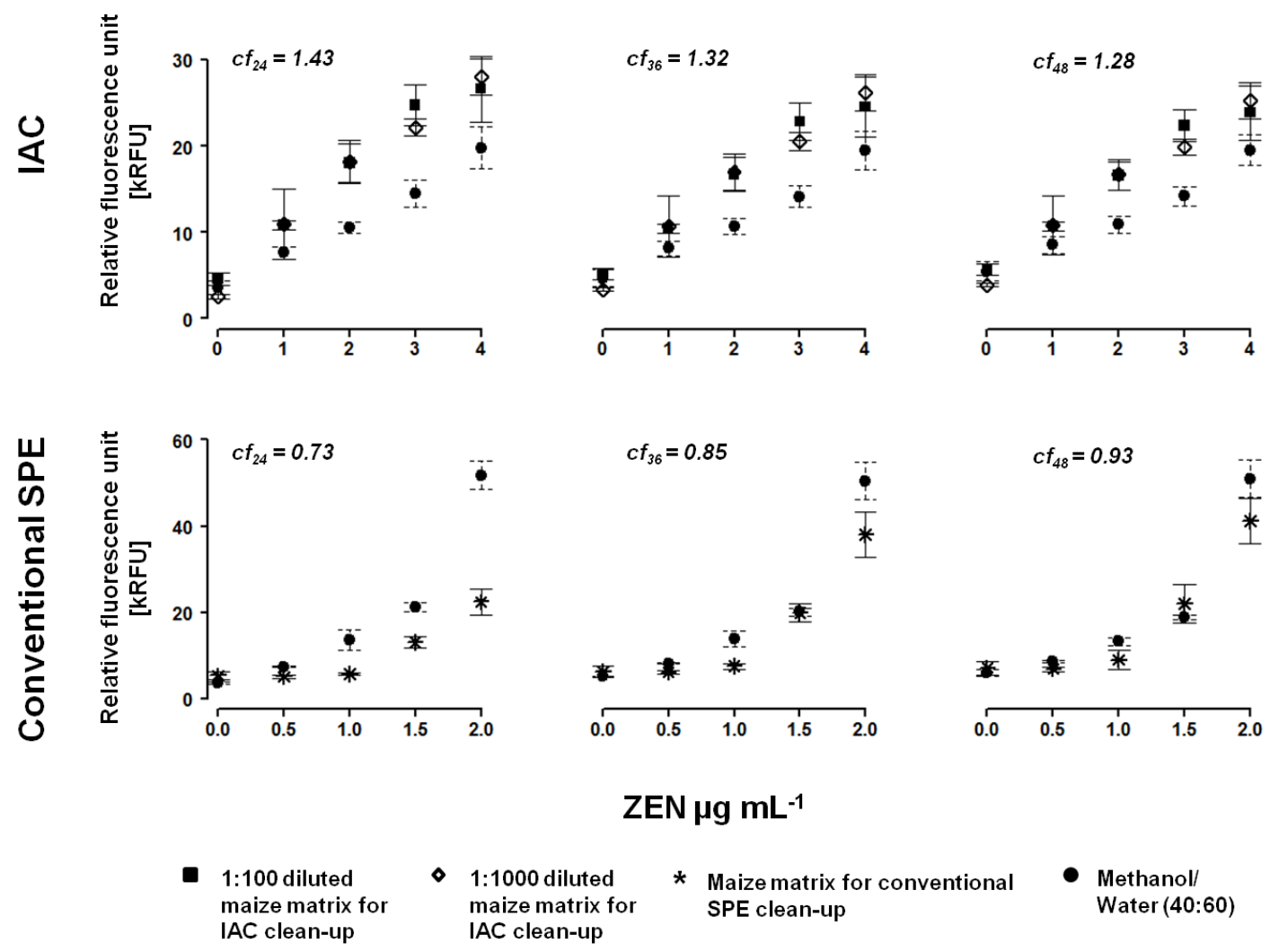

Fig. 4.8 Effect of maize matrix on fluorescence emission (given as mean relative fluorescence units (RFU) \pm standard deviation) of a G. roseum zes2::gfp culture (general procedure, section 4.2.4) exposed to zearalenone (ZEN) after clean-up with immunoaffinity columns (IAC) and conventional SPE (Bond Elut Mycotoxin) at time points (t) 24, 36 and 48 hours after addition of ZEN containing analyte; the matrix effect was assessed for undiluted samples (undiluted $=\mathrm{V} 0$ ), presented matrix factors fit to $\mathrm{VO}_{\mathrm{IAC}}$ or $\mathrm{V} 0_{\mathrm{BE}}$, factor of matrix correction at time point $\mathrm{t}\left(\mathrm{Cf}_{\mathrm{t}}\right)$ was calculated according to the formula given in section 4.2.10, $\mathrm{n}=3$

\subsubsection{Assay Kinetics in Alternative Procedure with Direct Exposure of G. roseum zes2::gfp Spores to ZEN}

The general procedure, including a pre-cultivation of G. roseum and addition of analyte after $48 \mathrm{~h}$, is comparatively time-consuming. Therefore, a simplified procedure (alternative procedure), comprising a direct exposure of G. roseum zes $2:: g f p$ spores to ZEN, was assessed. The highest fluorescence emission was observed at time point $60 \mathrm{~h}$ after initiation. As specified for the first procedure, the time point of maximum response was assured as optimum for quantitative purposes. The response of the G. roseum zes2::gfp strain (without preincubation) towards ZEN after 36, 48 and $60 \mathrm{~h}$ is presented in Fig. 4.9. Fluorescence readings were also performed at earlier time points, but before $24 \mathrm{~h}$, the fungal development was not visible and fluorescence signal was not exceeding background signal. For the 
alternative/direct exposure procedure, a linear response in fluorescence emission to ZEN was observed for a concentration range between $0.25 \mu \mathrm{g} \mathrm{mL}^{-1}$ and $2 \mu \mathrm{g} \mathrm{mL} \mathrm{m}^{-1}$. Under the tested conditions, a saturation of the recombinant strain with ZEN was observed for concentrations above $2 \mu \mathrm{g} \mathrm{mL}^{-1}$. Calculated LOD at time point $60 \mathrm{~h}$ was $0.01 \mu \mathrm{g} \mathrm{ZEN} \mathrm{ml}^{-1}$ in medium and LOQ was determined to be $0.04 \mu \mathrm{g} \mathrm{ZEN} \mathrm{mL}^{-1}$ in medium.

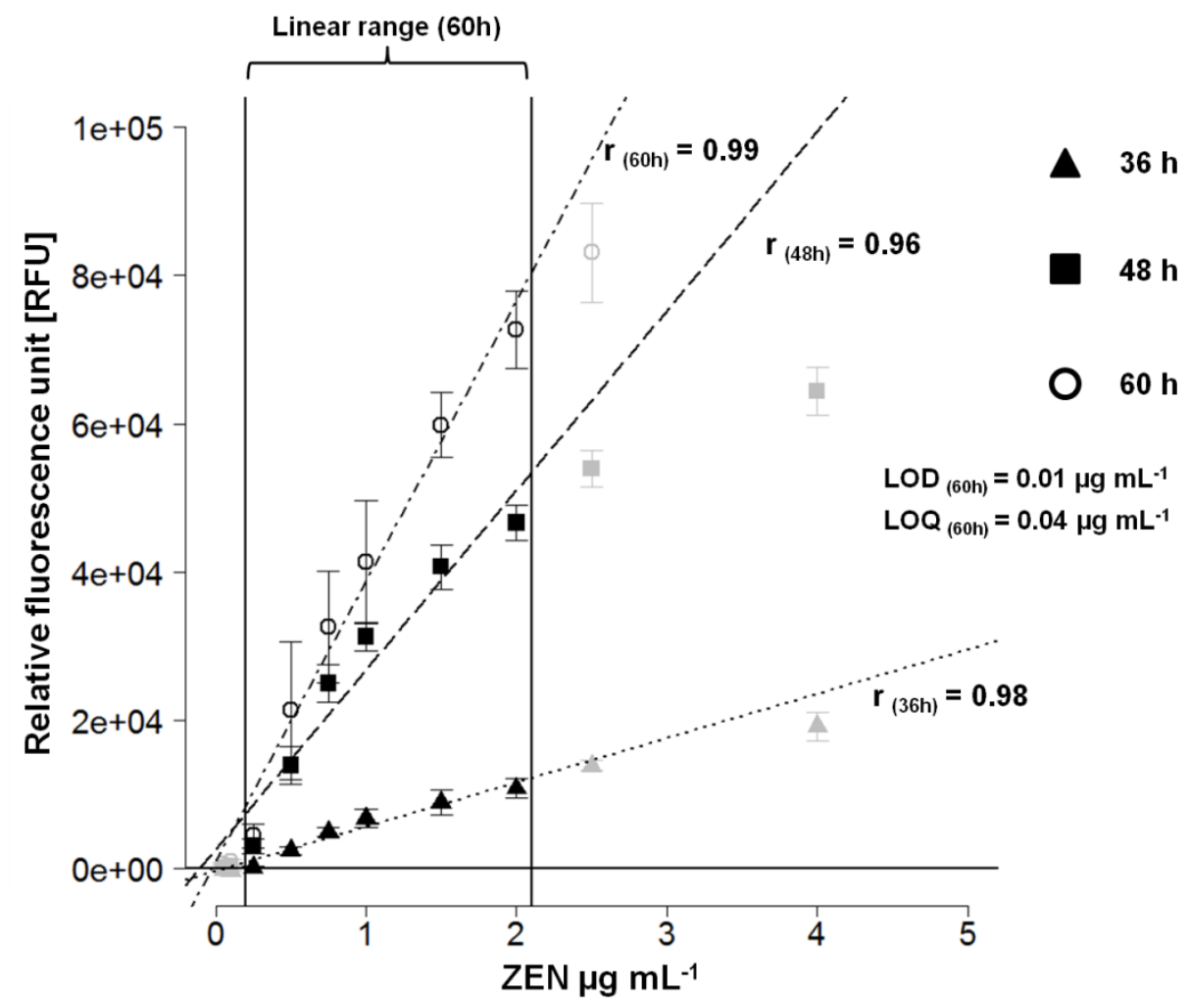

Fig. 4.9 Response of G. roseum zes2::gfp strain (alternative procedure, handling is described in section 4.2.7) after 36,48 and $60 \mathrm{~h}$ of exposition to zearalenone (ZEN), maximum response was observed at time point $60 \mathrm{~h}$, linear response to ZEN was observed in a range between $0.25 \mathrm{\mu g} \mathrm{mL}^{-1}$ and $2 \mu \mathrm{g} \mathrm{mL}^{-1}$ in medium, $\mathrm{n}=4$

\subsubsection{Quantification of ZEN in Field Samples}

The accuracy of the present bioassay for the detection of ZEN in complex matrices (operated in different modes), compared to expensive but precise HPLC-MS reference data was evaluated. HPLC-MS analysis revealed a broad spectrum of ZEN concentrations in the tested set of maize field samples $(n=17)$. ZEN concentrations were in the range between $0.9 \mathrm{mg} \mathrm{kg}^{-1}$ and $90 \mathrm{mg} \mathrm{kg}^{-1}$ dry matter. After clean-up with IAC columns, the predicted ZEN concentrations by G. roseum zes $2: \because g f p$ bioassay correlated strongly $(\mathrm{r}=0.85)$ with the results of liquid chromatography. The correlation is presented in Fig. 4.10. 

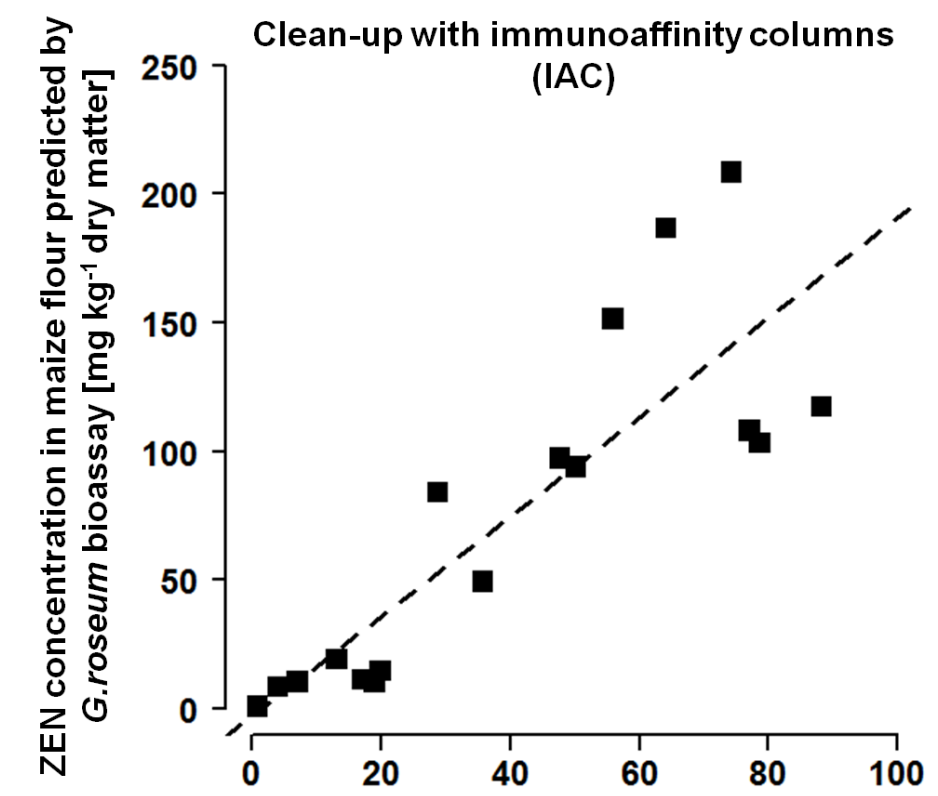

Fig.4.10 Strong correlation between zearalenone (ZEN) concentration in maize field samples (after clean-up with IAC columns) determined by G. roseum bioassay and by HPLC-MS ( $r=0.85$, coefficients of the linear model: intercept $=$ -2.64 , slope $=1.94), n=17$

ZEN concentration in maize flour determined by HPLC-MS [ $\mathrm{mg} \mathrm{kg}^{-1}$ dry matter]

After simpler clean-up with conventional SPE-columns (only 7 samples), the ZEN concentrations in contaminated maize field samples predicted by G. roseum zes $2:: g f p$ bioassay correlated only with $r=0.73$ with the determined ZEN concentrations by HPLC-MS (see Fig. 4.11).

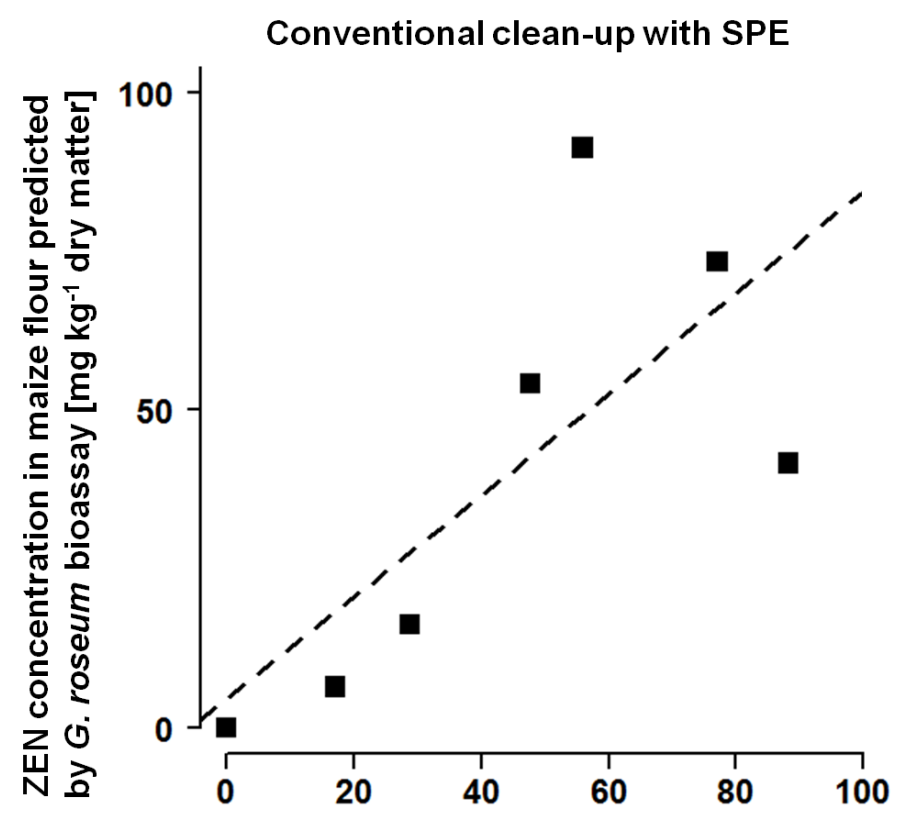

Fig. 4.11 Correlation between zearalenone (ZEN) concentration in maize field samples (after clean-up with conventional SPE columns, Bond Elut Mycotoxin) determined by G. roseum bioassay and by HPLC-MS $(r=$ 0.73 , coefficients of the linear model: intercept $=4.58$, slope $=$ $0.80), \mathrm{n}=7$

ZEN concentration in maize flour determined by HPLC-MS [mg kg-1 dry matter] 
Because of its time-saving properties, the alternative procedure, comprising a direct exposure of G. roseum zes $2:: g f p$ spores to ZEN, was tested for the assessment of ZEN in field samples of maize and wheat. Unexpectedly, the tested alternative procedure turned out to be improper for semi-quantitative purposes in context with these matrices because the fluorescence emission, as response of G. roseum zes $2:: g f p$ strain towards ZEN, exceeded background signal only for concentrations above $2 \mu \mathrm{g} \mathrm{ml}^{-1}$ in both undiluted matrices and only at late time points (> $60 \mathrm{~h}$ after addition of ZEN containing analyte). To reduce inhibitory matrix effects of maize and wheat, a clean-up of crude extracts with $1 \times$ volume ethyl acetate was tested $(\mathrm{pH}$ adjusted as required). This clean-up turned out to be not suitable for semi-quantitative purposes as well (data not shown).
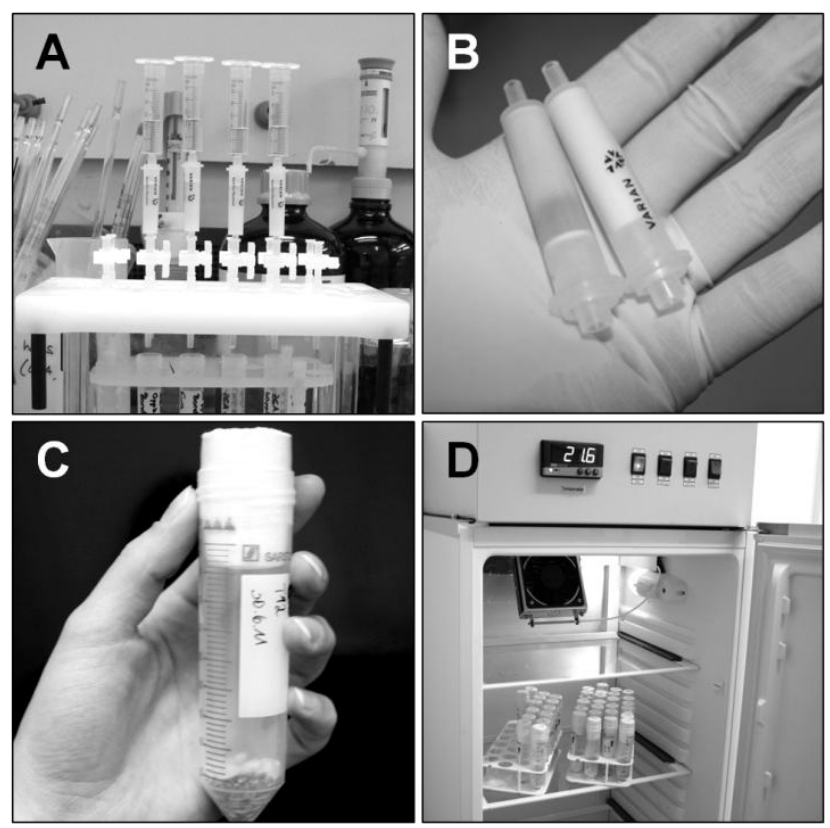

Fig. 4.12 Sample preparation for G. roseum bioassay, (A) conventional solidphase extraction (SPE) with Bond Elut Mycotoxin columns fixed to a vacuum chamber, (B) staining of SPE columns after use, left column was used for a sample, which was profoundly infected with F. graminearum, right column was used for a healthy maize sample, (C) in vitro culture on rice medium for fungal strain screening, cotton stoppers were used to facilitate gasexchange (D) incubator with space-saving strain cultures in centrifuge tubes

\subsubsection{Fungal Strain Screening}

The use of the present G. roseum bioassay for a qualitative high-throughput fungal strain screening for ZEN production was examined. According to HPLC-MS reference measurements, all tested fungal isolates $(n=38)$ were addressed properly to the group of ZEN producers and non-producers by G. roseum bioassay (after SPE clean-up). An overview of the assignment is given in Tab. 4.2. The fluorescence response of the recombinant strain towards ZEN in culture extracts at time point $36 \mathrm{~h}$ revealed a strong correlation with determined ZEN concentrations in extracts as determined by HPLC-MS $(r=0.93)$. The relation is presented in Fig. 4.13. 


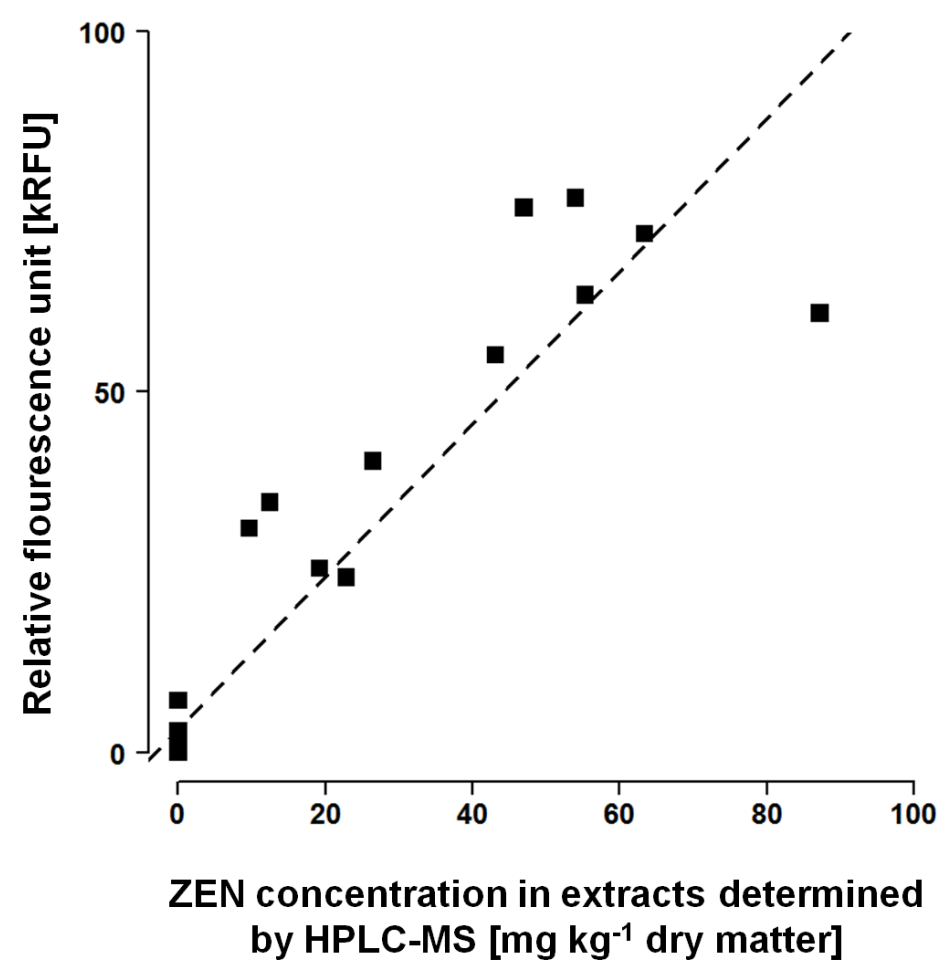

Fig. 4.13 Strong correlation between fluorescence response of G. roseum zes2::gfp strain towards zearalenone (ZEN) (expressed as relative fluorescence units, after SPE clean-up) and determined concentrations of ZEN in culture extracts with HPLC-MS $(r=0.93), n=38$

The response of the G. roseum zes2::gfp strain towards crude solvent extracts from in vitro cultures ( 9 selected isolates) was tested, to investigate the applicability of a simplified screening procedure without SPE clean-up. In this case as well, the assignment of fungal strains to the group of ZEN producers and non-producers, following the same criteria as described earlier, was possible from time point $36 \mathrm{~h}$ after initiation. 
Tab. 4.2 Assignment of 38 fungal strains to the group of zearalenone (ZEN) producers (labeled with '+') and non-producers (labeled with '-9) by G. roseum zes2::gfp bioassay and HPLC-MS

\begin{tabular}{|c|c|c|c|}
\hline \multirow[b]{2}{*}{ Fungal species } & \multirow[b]{2}{*}{ Isolate No./ Code* } & \multicolumn{2}{|c|}{$\begin{array}{c}\text { Identified as ZEN-producer }(+) \text { / no ZEN- } \\
\text { producer }(-)\end{array}$} \\
\hline & & Bioassay & HPLC-MS \\
\hline \multirow[t]{3}{*}{ Fusarium avenaceum } & DSM 62161 & - & - \\
\hline & CBS 121.73 & - & - \\
\hline & DSM 21724 & - & - \\
\hline F. culmorum & Fc 4.0 & + & + \\
\hline \multirow[t]{2}{*}{ F. equiseti } & O90 & - & - \\
\hline & $\mathrm{O} 20$ & - & - \\
\hline \multirow[t]{13}{*}{ F. graminearum } & FG210.1 & + & + \\
\hline & FG71 & + & + \\
\hline & $102.3 \mathrm{wt}$ & + & + \\
\hline & FG 7-34 & + & + \\
\hline & FG $201.1 \mathrm{wt}$ & - & - \\
\hline & FG 108.1 wt & + & + \\
\hline & FG 104.2 wt & + & + \\
\hline & FG 203.1 wt & + & + \\
\hline & FG 210 & + & + \\
\hline & FG 106.2 wt & + & + \\
\hline & $\mathrm{FG} 211.1 \mathrm{wt}$ & + & + \\
\hline & Fg 7-28 wt & + & + \\
\hline & $\mathrm{Fg} \mathrm{7-29} \mathrm{wt}$ & + & + \\
\hline \multirow[t]{2}{*}{ F. oxysporum } & DSM 2018 & - & - \\
\hline & DSM 62291 & - & - \\
\hline \multirow[t]{2}{*}{ F. poae } & FS 41 & - & - \\
\hline & F448 & - & - \\
\hline \multirow[t]{4}{*}{ F. proliferatum } & DSM 764 & - & - \\
\hline & DSM 62267 & - & - \\
\hline & DSM 62261 & - & - \\
\hline & DSM 63267 & - & - \\
\hline \multirow[t]{2}{*}{ F. subglutinans } & CBS 215.76 & - & - \\
\hline & PK 2-17-7 & - & - \\
\hline \multirow[t]{2}{*}{ F. tricinctum } & 063 & - & - \\
\hline & $\mathrm{O} 32$ & - & - \\
\hline \multirow[t]{5}{*}{ F. verticillioides } & Fv Ita 1 & - & - \\
\hline & FRC M-8114 & - & - \\
\hline & DSM 62264 & - & - \\
\hline & GF A & - & - \\
\hline & GF F & - & - \\
\hline \multirow[t]{2}{*}{ Trichoderma harzianum } & T.s. & - & - \\
\hline & $\mathrm{T} 12$ & - & - \\
\hline
\end{tabular}

* Isolate No. / Codes are specified in Tab. 4.1 


\subsection{Discussion}

A sensitive and specific bioassay for the detection of estrogenic ZEN and its derivates in maize field samples was described. The employed G. roseum zes2::gfp mutant selectively responded to the estrogenic mycotoxin ZEN and its most critical metabolite $\alpha$-ZOL (see Fig. 4.5). The response was dose-dependant (see Fig. 4.7), as shown by Utermark \& Karlovsky (2006), who described the use of a microplate reader for fluorescence measurements. The assay was suitable for a qualitative detection of ZEN in a broad concentration range (Fig. 4.7, Fig. 4.9) and for a quantitative evaluation of ZEN contamination of maize field samples in the range of $0.9 \mathrm{mg} \mathrm{kg}^{-1}$ to $90 \mathrm{mg} \mathrm{kg}^{-1}$. ZENconcentrations, determined with the help of the G. roseum bioassay and by HPLC-MS, correlated with $r=0.85$ (Fig. 4.10).

Clean-up of sample extracts and conditioning of the G. roseum zes $2: \because g f p$ strain are important for an accurate evaluation of toxin contamination. Our results indicate that conventional SPE columns are less suitable to reduce the inhibitory effect of maize matrix on the indicator strain. We assume that the maize matrix inhibited the growth of the G. roseum zes $2: \because g f p$ strain or the substrate accessibility was affected by matrix compounds. A clean-up step on IAC was more efficient than conventional SPE to reduce matrix effects. The beneficial use of an IAC clean-up for liquid chromatography analysis of ZEN was also reported by Erbs et al. (2007), Visconti \& Pascale (1998) and Scott \& Trucksess (1997). A new clean-up method, based on dynamic covalent hydrazine chemistry (DCHC) (Siegel et al., 2010), might also be useful in combination with the present bioassay. Comparison of direct exposure of the G. roseum zes $2:: g f p$ spores to ZEN (section 4.2.7) and pre-incubation of spores for $48 \mathrm{~h}$ before ZEN was added (section 4.2.4) showed that the latter was more suitable for the analysis of field sample extracts. We assume that the pre-incubation phase provides larger fungal surface due to the growth of young reactive hyphae (fungal development is visible from that time point on), which might fasten the recognition and, consequently, the fluorescence response to ZEN.

Beside the aforementioned semi-quantitative approach in field samples, we successfully applied the G. roseum zes $2:: g f p$ bioassay for a qualitative discrimination of ZEN-producing fungi from others. For this purpose, a conventional SPE clean-up, after solvent extraction of ZEN from fungal cultures (rice kernels as substrate), was sufficient. Preliminary results, based on a small number of samples, indicate that a SPE clean-up can actually be omitted for a qualitative screening on rice. Furthermore, the screening of fungal strains for ZEN production 
can be carried out without pre-incubation. Apart from the qualitative detection of ZEN in fungal cultures, we observed a strong correlation between fluorescence response of the G. roseum zes $2:: g f p$ bioassay after SPE clean-up and ZEN concentration determined by HPLC-MS ( $\mathrm{r}=0.93$, Fig. 4.13). Because extracts of maize matrix required IAC clean-up, these results indicate that rice extracts inhibit the $G$. roseum assay to a lower extent than maize.

This is the first report of a ZEN-bioassay, applicable for a screening of field samples that selectively corresponds to ZEN and its reductive metabolite $\alpha$-ZOL, but no other estrogenic compounds. All previously described bioassays for ZEN were not specific for Fusarium mycoestrogens, but responded to a broad spectrum of compounds, like genistein, $17 \beta$-estradiol and other hormonal substances (Winter et al., 2008; Bovee et al., 2004; Mitterbauer et al., 2003; Mayr et al., 1992; Welshons et al., 1990). Considering the fact that ZEN causes severe problems in animal production and the instance that the protein-source in animal food is usually based on legumes that release estrogens to a high extent, the application of such unspecific estrogen bioassays for a mycotoxin risk management in agriculture is questionable.

The described bioassay for ZEN can be accomplished with common laboratory equipment and consumables, such as chemicals for media, glassware and organic solvents. In comparison to common bench-top liquid chromatography systems, the costs of analysis are low. Hence, it might be suitable for the application in developing countries and as a risk assessment tool for non-governmental organizations. Under certain conditions (heating of the lid can be switched off), a real-time PCR cycler can be used instead of a microplate reader (Utermark \& Karlovsky, 2006), extending the applicability of the method to laboratories equipped for DNA analysis.

Apart from the quantitative and qualitative application for ZEN detection, the described bioassay could serve as a model approach for the specific detection of metabolites in biological systems by an employment of microorganisms with abilities to recognize and, respectively, transform or decompose these metabolites. Nevertheless, the approach requires a distinct knowledge about interactions, biological pathways and the molecular level. 


\section{Acknowledgements}

We acknowledge the working group of Prof. v. Tiedemann (Plant Pathology section, University of Goettingen, Germany) for the provision of fungal isolates and Dr. Sabine Nutz for the support with maize material from field trials.

\section{References}

Ahamed S, Foster JS, Bukovsky A, Wimalasena J (2001) Signal transduction through the ras/Erk pathway is essential for the mycoestrogen zearalenone-induced cellcycle progression in MCF-7 cells. Molecular Carcinogenesis 30:88-98.

Anonymus (2007) Commission regulation (EC) no. 1126/2007amending Regulation (EC) No $1881 / 2006$ setting maximum levels for certain contaminants in foodstuffs as regards Fusarium toxins in maize and maize products. Official Journal of the European Union 255:14-17.

Bai G-H, Shaner G (1996) Variation in Fusarium graminearum and cultivar resistance to wheat scab. Plant Disease 80:975-979.

Bennett GA, Nelsen TC, Miller BM (1994) Enzyme-linked immunosorbent assay for detection of zearalenone in corn, wheat, and pig feed: collaborative study. Journal of AOAC International 77:1500-1509.

Bennett JW, Klich M (2003) Mycotoxins. Clinical Microbiology Reviews 16:497-516.

Bottalico A, Perrone G (2002) Toxigenic Fusarium species and mycotoxins associated with head blight in small-grain cereals in Europe. European Journal of Plant Pathology 108:611-624.

Bovee TFH, Helsdingen RJR, Rietjens IMCM, et al. (2004) Rapid yeast estrogen bioassays stably expressing human estrogen receptors $\alpha$ and $\beta$, and green fluorescent protein: a comparison of different compounds with both receptor types. The Journal of Steroid Biochemistry and Molecular Biology 91:99-109.

Caldwell RW, Tuite J, Stob M, Baldwin R (1970) Zearalenone production by Fusarium species. Applied Microbiology 20:31-34.
Cha S-H, Kim S-H, Bischoff K, et al. (2012) Production of a highly group-specific monoclonal antibody against zearalenone and its application in an enzyme-linked immunosorbent assay. Journal of Veterinary Science 13:119.

D’Mello JPF, Placinta CM, Macdonald AMC (1999) Fusarium mycotoxins: a review of global implications for animal health, welfare and productivity. Animal Feed Science and Technology 80:183-205.

el-Sharkawy S, Abul-Hajj YJ (1988) Microbial cleavage of zearalenone. Xenobiotica 18:365-371.

Erbs M, Hartmann N, Bucheli TD (2007) Determination of the cross-reactivities for alpha-zearalenol, beta-zearalenol, zearalanone, alpha-zearalanol, and betazearalanol on three commercial immunoaffinity columns targeting zearalenone. Journal of AOAC International 90:1197-1202.

Gromadzka K, Waskiewicz A, Chelkowski J, Golinski P (2008) Zearalenone and its metabolites: occurrence, detection, toxicity and guidelines. World Mycotoxin Journal 1:209-220.

Hagler WM, Mirocha CJ, Pathre SV, Behrens JC (1979) Identification of the naturally occurring isomer of zearalenol produced by Fusarium roseum "Gibbosum" in rice culture. Applied and Environmental Microbiology 37:849-853.

Jackson LS, Bullerman LB (1999) Effect of processing on Fusarium mycotoxins. Advances in Experimental Medicine and Biology 459:243-261.

Kuiper GGJM (1998) Interaction of estrogenic chemicals and phytoestrogens with Estrogen Receptor. Endocrinology 139:4252-4263. 
Kuiper-Goodman T, Scott PM, Watanabe H (1987) Risk assessment of the mycotoxin zearalenone. Regulatory Toxicology and Pharmacology 7:253-306.

Leffers H, Naesby M, Vendelbo B, et al. (2001) Oestrogenic potencies of Zeranol, oestradiol, diethylstilboestrol, Bisphenol-A and genistein: implications for exposure assessment of potential endocrine disrupters. Human Reproduction 16:10371045.

Lioi M., Santoro A, Barbieri R, et al. (2004) Ochratoxin A and zearalenone: a comparative study on genotoxic effects and cell death induced in bovine lymphocytes. Mutation Research/Genetic Toxicology and Environmental Mutagenesis 557:19-27.

Logrieco A, Mulé G, Moretti A, Bottalico A (2002) Toxigenic Fusarium species and mycotoxins associated with maize ear rot in Europe. European Journal of Plant Pathology 108:597-609.

Marin DE, Taranu I, Burlacu R, Tudor DS (2010) Effects of zearalenone and its derivatives on the innate immune response of swine. Toxicon 56:956-963.

Massart F, Saggese G (2010) Oestrogenic mycotoxin exposures and precocious pubertal development. International Journal of Andrology 33:369-376.

Mayr U, Butsch A, Schneider S (1992) Validation of two in vitro test systems for estrogenic activities with zearalenone, phytoestrogens and cereal extracts. Toxicology 74:135149 .

McMullen M, Jones R, Gallenberg D (1997) Scab of wheat and barley: A re-emerging disease of devastating impact. Plant Disease 81:1340-1348.

Miller JD (2008) Mycotoxins in small grains and maize: Old problems, new challenges. Food Additives \& Contaminants: Part A 25:219-230.

Mitterbauer R, Weindorfer $\mathrm{H}$, Safaie $\mathrm{N}$, et al. (2003) A sensitive and inexpensive yeast bioassay for the mycotoxin zearalenone and other compounds with estrogenic Activity. Applied and Environmental Microbiology 69:805-811.
Morandi MAB, Sutton JC, Maffia LA (2000) Effects of host and microbial factors on development of Clonostachys rosea and control of Botrytis cinerea in rose. European Journal of Plant Pathology 106:439-448.

Othmen ZO-B, Golli EE, Abid-Essefi S, Bacha H (2008) Cytotoxicity effects induced by zearalenone metabolites, $\alpha$-zearalenol and $\beta$-zearalenol, on cultured Vero cells. Toxicology 252:72-77.

Pfohl-Leszkowicz A, Chekir-Ghedira L, Bacha H (1995) Genotoxicity of zearalenone, an estrogenic mycotoxin: DNA adduct formation in female mouse tissues. Carcinogenesis 16:2315-2320.

Reid LM, Hamilton R 1., Mather DE (1995) Effect of macroconidial suspension volume and concentration on expression of resistance to Fusarium graminearum in Maize. Plant Disease 79:461-466.

Scott PM, Trucksess MW (1997) Application of immunoaffinity columns to mycotoxin analysis. Journal of AOAC International 80:941-949.

Siegel D, Andrae K, Proske M, et al. (2010) Dynamic covalent hydrazine chemistry as a selective extraction and cleanup technique for the quantification of the Fusarium mycotoxin zearalenone in edible oils. Journal of Chromatography A 1217:2206-2215.

Songsermsakul P, Sontag G, Cichnamarkl M, et al. (2006) Determination of zearalenone and its metabolites in urine, plasma and faeces of horses by HPLC-APCI-MS. Journal of Chromatography B 843:252-261.

Stopper H, Schmitt E, Kobras K (2005) Genotoxicity of phytoestrogens. Mutation Research/Fundamental and Molecular Mechanisms of Mutagenesis 574:139-155.

Sutton JC, Li DW, Peng G, et al. (1997) Gliocladium roseum a versatile adversary of Botrytis cinerea in crops. Plant Disease 81:316-328.

Tanaka T, Hasegawa A, Yamamoto S, et al. (1988) Worldwide contamination of cereals by the Fusarium mycotoxins nivalenol, deoxynivalenol, and zearalenone. 1 . Survey of 19 countries. Journal of Agricultural and Food Chemistry 36:979983. 
Tanaka T, Hasegawa A, Matsuki Y, et al. (1985) Rapid and sensitive determination of zearalenone in cereals by highperformance liquid chromatography with fluorescence detection. Journal of Chromatography A 328:271-278.

Thevis M, Fußhoeller G, Schaenzer W (2011) Zeranol: doping offence or mycotoxin? A case-related study. Drug Testing and Analysis 3:777-783.

Urry WH, Wehrmeister HL, Hodge EB, Hidy PH (1966) The structure of zearalenone. Tetrahedron Letters 7:3109-3114.

Utermark J, Karlovsky P (2007) Role of zearalenone lactonase in protection of Gliocladium roseum from fungitoxic effects of the mycotoxin zearalenone. Applied and Environmental Microbiology 73:637-642.

Utermark J, Karlovsky P (2006) Quantification of green fluorescent protein fluorescence using real-time PCR thermal cycler. BioTechniques 41:150-154.

Visconti A, Pascale M (1998) Determination of zearalenone in corn by means of immunoaffinity clean-up and highperformance liquid chromatography with fluorescence detection. Journal of Chromatography A 815:133-140.

Welshons WV, Rottinghaus GE, Nonneman DJ, et al. (1990) A sensitive bioassay for detection of dietary estrogens in animal feeds. Journal of Veterinary Diagnostic Investigation 2:268-273.

Winter P, Nau H, Lampen A, Kamphues J (2008) Detection of estrogenically active substances in diets for sows by an in vitro bioassay supported by HPLC analysis. Journal of Animal Physiology and Animal Nutrition 92:337-344.

Yu Z, Zhang L, Wu D, Liu F (2005) Anti-apoptotic action of zearalenone in MCF-7 cells. Ecotoxicology and Environmental Safety 62:441-446.

Zinedine A, Soriano JM, Moltó JC, Mañes J (2007) Review on the toxicity, occurrence, metabolism, detoxification, regulations and intake of zearalenone: An oestrogenic mycotoxin. Food and Chemical Toxicology 45:1-18. 


\section{General Discussion}

The present study was concerned with two main objectives. The first aim was the development of a new approach for the fast recognition of Fusarium infections of maize and wheat under natural conditions. For this purpose, we investigated the use of volatile organic compounds (VOCs) as infection markers for a classification among 'infected' and 'noninfected' ears. The second aim was the development of a specific high-throughput bioassay for the detection of zearalenone (ZEN), an estrogenic mycotoxin produced by several Fusarium spp., in agricultural commodities. In this case, we employed a recombinant strain of the mycoparasite Gliocladium roseum that is able to detoxify ZEN by a specific lactonase.

VOCs are low-molecular carbons with high vapor pressure and a high structural diversity. Almost every organism (i.e. plants, animals, microbes) releases volatiles, which are more or less specific to that organism under a certain environmental and physical condition (i.e. light, temperature, nutrition) (Holopainen \& Gershenzon, 2010; Kesselmeier \& Staudt, 1999). It is accepted that plants, especially herbs or medicinal plants, have a characteristic volatile profile and their volatile emission is regulated by the developmental stage (i.e. flowering) and by the stress situation of the individual plant (Dudareva et al., 2004). In this context, it has been reported that maize releases some volatiles, such as $(E)$ - $\beta$-caryophyllene, upon attack by herbivores or fungi (Smith et al., 2012; Piesik et al., 2011). The release of plant VOCs upon biotic stress is often involved in direct or indirect plant defense (Soliman \& Badeaa, 2002; Kessler \& Baldwin, 2001； Daferera et al., 2000； Dicke et al., 1990； Turlings et al., 1990; Baratta et al., 1998). Depending on the developmental stage, also fungi produce characteristic volatiles that might be advantageous during infection and competition (Kramer \& Abraham, 2011; Linton \& Wright, 1993). In the past, such fungal volatile signatures (i.e. 3-octanone, 3-octanol) were used for the recognition of fungal spoilage in storages or under in vitro conditions (Magan \& Evans, 2000). Up to now, only few reports have focused on the volatile profile of fungal infections on live plants, for example Perkowski et al. (2008) and Girotti et al. (2012), who monitored volatiles from cereals infected with F.culmorum and F. graminearum. Both groups emphasized the role of 
trichodiene as infection marker. Until now, the VOC emission of Fusarium infected maize ears has not been investigated under in vivo conditions.

In the present study, the volatile spectra released from Fusarium infected plants were monitored in the greenhouse or climate chamber to exclude natural infections with Fusarium spp. that would normally appear in the field (Henriksen \& Elen, 2005; Placinta et al., 1999; Parry et al. 1995). Ear infections were carried out with toxin producing Fusarium species, occurring in maize (F. graminearum, F. verticillioides, F. subglutinans) and in wheat (F. graminearum, F. avenaceum, F. poae) all over the world (Dorn et al., 2009; Stenglein, 2009; Osborne \& Stein, 2007; Logrieco et al., 2002; Bottalico \& Perrone, 2002). An endpoint monitoring of volatile profiles (endpoint (maize) $=24 \mathrm{dpi}$, endpoint (wheat) $=21 \mathrm{dpi}$ ) as well as time series with several intervals between inoculation and endpoint were conducted. Time series were performed to assess the earliest timepoint for a detection of specific markers indicating fungal infection. A static (SPME) and/or a dynamic system (non-destructive OLS with internal standard calibration), each followed by GC-MS analysis, were employed for the samplings. Both, SPME and OLS, techniques are well described in the literature for the investigation of VOCs from fungi (Jeleń, 2003; Demyttenaere et al., 2003) and small-grain cereals and maize (Hiltpold \& Turlings, 2008; Jeleń et al., 2003). The non-exhaustive SPME/GC-MS method, applied without calibration, is considered as a qualitative up to semiquantitative method (Vas \& Vékey, 2004). Difficulties and constraints in context with the SPME procedure (i.e. selectivity of adsorbent, humidity and temperature adjustment, determination of an adequate exposure time) and its quantitative use were discussed by Matich et al. (1996), Ai (1997) and Ouyang \& Pawliszyn (2008). Considering these aspects, we normalized the procedure of sample collection for SPME as well as sample mass, extraction time and temperature during extraction process. Furthermore, we compared the results of normalized dynamic and non-normalized static sampling and received a high correlation for sesquiterpeoid compounds $(r=1)$. Hence, we concluded that matrix effects are of minor importance and, consequently, used the SPME/GC-MS approach for quantitative comparisons between volatile profiles of infected ears with Fusarium spp. (single and mixed infection) and of non-infected ears.

On commercial hybrid maize ears, infected with strains of $F$. graminearum, $F$. verticillioides and $F$. subglutinans or with a mixture of $F$. graminearum and $F$. verticillioides strains, we identified a set of 27 volatile biomarkers to distinguish between 'healthy' and 'infected' ears as well as between different species of Fusarium (time point 24 dpi). Numerous volatile 
markers, identified via SPME/GC-MS, were also detected by non-destructive OLS/GC-MS. However, the used adsorbent material, activated charcoal, was selective for some molecules and did not capture all substances that were detected by SPME/GC-MS. Multiple layer absorption, as described by Liu et al. (2008) and Brancaleoni et al., (1999), or the parallel use of different adsorbents could have solved the problem of selectivity. Our investigations on summer wheat ears, infected with $F$. graminearum, $F$. poae and $F$. avenaceum and mixtures of $F$. graminearum with $F$. poae or $F$. avenaceum, revealed a set of 13 volatile markers for infection (at $21 \mathrm{dpi}$ ). VOC collections in time series showed that the identified volatile markers were largely present from the earliest time points of our investigations on (4 dpi in maize and 7 dpi in wheat). Under field conditions, where infection procedures may be temporally inhibited by unfavorable conditions for fungal growth (i.e. temperature and humidity), it seems possible that markers appear later.

The identified volatile markers in maize and wheat included a number of common 5 to 8 carbon containing volatiles $\left(\mathrm{C}_{5}-\mathrm{C}_{8}\right)$. These VOCs are widespread among plants and microbes (Combet et al., 2006; Hatanaka, 1993; Kaminśki et al., 1974). Unsurprisingly, the $\mathrm{C}_{5}-\mathrm{C}_{8}$ group, detected from Fusarium infected plant material, included compounds of probable fungal origin, such as 3-octanone and 3-octanol. These volatiles have been reported for other fungal genera than Fusarium (Kaminśki et al., 1974), such as Penicillum (Chitarra et al., 2005), Aspergillus, Phialophora (Sunesson et al., 1995) and Myrothecium (Banerjee et al., 2010), and could, therefore, not serve as specific markers for a Fusarium infection. Moreover, it was stated that these volatiles are general indicators for fungal spoilage in domestic homes and edibles (Polizzi et al., 2012; Schnuerer et al. 1999). Hence, a detection of such 'mushroom' volatiles in the field or during harvest processes would generally indicate a fungal contamination. Surprisingly, we detected 1-octen-3-ol, described as typical fungal volatile by Kamiński et al. (1972) and Kaminśki et al. (1974), in non-infected wheat samples. This volatile was also found in the head-space of non-infected wheat ear material by Birkett et al. (2004) and in barley (Hordeum vulgare L.) (Cramer et al., 2005), confirming that it might be released by the plant as well.

Other VOCs of the $\mathrm{C}_{5}-\mathrm{C}_{8}$ group, such as 2-heptanol, were often described as typical plant volatiles, i.e. GLVs (Rostás et al., 2006; Hatanaka, 1993). As expected, these compounds were detected with highest concentrations in non-infected maize and wheat plants (ears, inoculated with water only). Our experiments revealed that the more severe the fungal infection was $(F$. graminearum single and mixed treatment showed highest aggressiveness on 
wheat and maize) and the more advanced the infection process was (checked in time series) the less abundant were typical plant volatiles in both, infected wheat and maize. We assume that the reduction of typical plant volatiles upon Fusarium infection might be caused by water and nutrient depletion in the ear, as described by Miedaner (1997), but also by the disruption of plant metabolic pathways due to phytotoxicity of fungal secondary metabolites (i.e. disruption of protein synthesis in eukaryotic cells by trichothecenes) (Desjardins \& Hohn, 1997).

Beside those common $\mathrm{C}_{5}-\mathrm{C}_{8}$ volatiles, we detected a de novo synthesis of sesquiterpenes after Fusarium infection in both, maize and wheat. Volatile sesquiterpenes were the predominant class among identified markers in infected hybrid maize ears (18 SQTs, $67 \%$ of marker compounds, identified by SPME/GC-MS), whereas the wheat marker set contained only two SQTs out of 13 compounds. A group of five SQTs was identified as major signals in chromatograms of infected maize ears (present $\leq 8 \mathrm{dpi}$ ). The group was not detected in noninfected ears and consisted of $\alpha$ - and $\beta$-selinene, $\beta$-macrocarpene, $\beta$-bisabolene and trichodiene. Selinene derivatives were described as major components in the essential oil of many plant species, such as Apium graveolens ( $\beta$-selinene, Momin et al., 2000; Rao et al., 2000), Callicarpa macrophylla (Singh et al., 2010), Leptospermum scoparium, Kunzea ericoides (Porter \& Wilkins, 1999) and Hypericum linarioides ( $\alpha$-selinene, Cakir et al., 2005). It has been reported that selinene exerts antimicrobial effects (Joshi, 2013; Vukovic et al., 2007) and its inhibitory effect on Fusarium spp. was discussed by Cakir et al. (2005). Thus, we assume that a de novo synthesis and release of selinene derivatives is part of the maize plant defense response against Fusarium infection. Apart from this, there are also evidences that $\alpha$ - and $\beta$-selinene might be of fungal origin, as reported by Polizzi et al. (2012) from Periconia britannica. The two compounds, $\beta$-macrocarpene and $\beta$-bisabolene, are volatile precursors in the synthesis of zealexins, a class of phytoalexins that was recently described by Huffaker et al. (2011) in young maize stems upon biotic stress. This indicates that both SQTs contribute to plant defense as well. In this context, we demonstrated the induction of nonvolatile zealexins A1 and A3 in Fusarium infected maize ears and, consequently, confirm the induction of this defense pathway. The last sesquiterpenoid of the major group of volatile markers from infected maize was trichodiene, the well known volatile precursor of trichothecenes (i.e. DON, NIV) (Demyttenaere et al., 2004; Jeleń et al., 1997). It was exclusively detected in $F$. graminearum infected maize but not in wheat ears, treated with the same species. This was surprising, because others reported trichodiene from wheat, 
contaminated with trichothecene producing Fusarium spp. (Perkowski et al., 2008). One option might be that the transformation of trichodiene to non-volatile products was efficient and, consequently, the release of the volatile intermediate was minimized. Another explanation might be that the release of trichodiene from wheat was limited to very early time points (present $\leq 7 \mathrm{dpi}$ ) that were not investigated in our study. Further terpenoid volatiles, released from infected maize were (+)-aromadendrene, (E)- $\beta$-farnesene and $\alpha$-muurolene, which were already reported in plants upon biotic stress (Sabater-Jara et al., 2010; Soković et al., 2009; Schnee et al., 2006; Bernasconi et al., 1998). Two sesquiterpenes, (+)-cycloisosativene and $\alpha$-ylangene, were released by non-infected maize ears at highest levels and were absent or found in minor concentrations in the headspace of Fusarium infected tissue (at $24 \mathrm{dpi}$ ). Time series from 4 to 24 dpi illustrated that these signals decreased quantitatively over time with disease progress in Fusarium infected ears (similar to $\mathrm{C}_{5}-\mathrm{C}_{8}$ plant VOCs). Both compounds were described in other plants than maize before and might also possess antimicrobial features (Lin et al., 2012; Chen et al., 2012; Hosni et al., 2011). The remaining 6 sesquiterpenoid compounds in maize and the two SQTs in infected wheat were not identified.

Among all volatile compounds, identified as markers for Fusarium infection of wheat and maize, the terpenoid compounds were the most infection specific. The described profiles of volatile markers in hybrid maize and in summer wheat offered the possibility to distinguish between infected and non-infected ears and the marker set in maize enabled to discriminate between the most important Fusarium species on maize, F. graminearum and F. verticillioides, the main causal agents of red and pink ear rot. The majority of volatile markers for Fusarium infection was detected at an early time point of 4 - 8 dpi. At this time stage, a visual detection of fusarioses in maize fields is hardly possible without mechanical injuring of the ear. Thus, we assume that specific volatile markers could serve as a tool for an early, non-destructive detection of fusarioses in the field. Apart from this, the field-detection of volatiles would immediately reveal information about the infection status, because further preparative steps are not necessary. In contrast to this, the common detection techniques for Fusarium infection and their toxic secondary metabolites, such as real-time PCR and HPLC-MS, require a laborious sample preparation and analysis. Nevertheless, the transfer of the described technology to the field requires portable detectors with adequate sensitivity. Until now, there are several devices available, such as E-noses that were already used for the prediction of fumonisin contamination in maize (Gobbi et al., 2011; Falasconi et al., 2005) or 
detection of fungal spoilage (Keshri et al., 1998), but also ion mobility spectrometers (IMS), which posses a high sensitivity (Kolakowski \& Mester, 2007; Borsdorf \& Eiceman, 2006). With calibrated portable detectors (i.e. incorporated in standard processes) the present technology could be used for field measurements and for the monitoring of harvest or post-harvest processes (i.e. storage, transport or feed production). A classification into 'healthy' and 'contaminated' by volatile profiling could serve as decision guidance for the application of pesticides as part of good agricultural practice and the selection of a suitable marketing strategy (i.e. human consumption, animal feed, energy use).

Our results from in vitro experiments with Fusarium spp. growing on autoclaved maize, wheat and rice kernels revealed no comparable VOC-profiles to greenhouse trials. The results indicate that volatile infection markers, applicable in the field, should be obtained under natural conditions (considering the volatile background of the live plant that releases relevant VOCs upon pathogen attack).

In future, it has to be clarified whether the described volatile biomarkers for Fusarium infection are constantly expressed among cultivars, as expected from our experiments with the dwarf maize variety, or if they are cultivar-specific. So far, an effect of the cultivar was reported for other agricultural and horticultural crops (i.e. potato, tomato, wine) (Vilanova et al., 2012; Berna et al., 2004; Duckham et al., 2002). Apart from this, also the intraspecies variation of the pathogen (i.e. mixture of released secondary metabolites, chemotypes) might affect the volatile spectra in infected crops. Of particular interest is the specificity of the described set of volatiles to an infection with Fusarium spp. As discussed earlier, our volatile markers included common fungal volatiles (i.e. 3-octanone) that would probably also appear upon infection with other fungal pathogens (i.e. Ustilago maydis in maize, Puccinia striiformis in wheat).

In addition to the analysis of VOCs, we monitored the mycotoxin contamination in infected plants by HPLC-MS. Hybrid maize samples were checked for fumonisins, DON, NIV and ZEN and summer wheat samples were analyzed for DON, NIV, ZEN, enniatins and BEA. Our analysis revealed that the concentrations of mycotoxins in infected ear material were exceptionally high in both, maize and wheat. This was observed especially for NIV and DON in F. graminearum infected wheat ears (single infection). In fields, naturally infected with Fusarium spp. (usually appearing as multiple species infection), it is not expected to find such high concentrations (Bottalico \& Perrone, 2002; Placinta et al., 1999). We found that the 
mycotoxin concentrations were considerably higher in single infection treatments than in mixed infection treatments and assume that the reduced mycotoxin levels in the mixed treatment are caused by interspecies competition. This aspect was also discussed by Xu et al. (2007) and Cooney et al. (2001) and is supported by our findings in wheat. In this case we reported a significant suppression of mycotoxin and biomass production by $F$. avenaceum, provided that the species grew together with $F$. graminearum.

Beside mycotoxins and zealexins, we analyzed a third class of non-volatile secondary metabolites in hybrid maize ears, the plant oxylipins. We found a significant up-regulation of these compounds upon pathogen attack (especially in F. graminearum infected ears). Oxylipins are known to possess antimicrobial properties and act as chemical defense agents close the site of pathogen attack (Goebel \& Feussner, 2009; Prost et al., 2005; Farmer et al., 2003; Blée, 2002). So far, it was emphasized that they have a signaling function in the plant (Eckardt, 2008). Since the oxylipin formation is initiated by multiple stress factors (Howe \& Schilmiller, 2002), it is not likely that their up-regulation is specific to Fusarium infection.

In the present study, we reported that a classification among infected and non-infected plants is possible based on the analysis of non-volatile secondary metabolites, such as toxins, zelaexins and oxylipins. Nevertheless, their analysis is time-consuming and expensive (i.e. due to sample preparation and costs of bench-top instruments), therefore, these compounds are not suitable for a broad application during industrial processes. In contrast to this, the monitoring of volatiles might be realized with calibrated portable devices, without impairing the plant or the product.

Apart from the detection of fungal presence on live plants by volatile analysis, we reported for the first time a sensitive and specific high-throughput bioassay for the detection of zearalenone and its most critical metabolite $\alpha$-ZOL in maize field samples. For this purpose, we employed a zes $2:: g f p$ mutant strain of G. roseum that selectively corresponds to the estrogenic mycotoxin. We observed a dose-dependant response (fluorescence emission of the mutant strain), which was also reported by Utermark \& Karlovsky (2006), who compared the use of a microplate reader and a conventional real-time PCR cycler for fluorescence measurements. The assay was suitable for a ZEN concentration range in field samples between $0.9 \mathrm{mg} \mathrm{kg}^{-1}$ to $90 \mathrm{mg} \mathrm{kg}^{-1}$. Quantitative results by HPLC-MS and zes $2: \because g f p$ bioassay revealed a high correlation $(\mathrm{r}=0.85)$. 
We optimized the extraction and clean-up process of ZEN from complex matrixes, such as maize flour from Fusarium contaminated ears. A clean-up step with immunoaffinity columns $\left(Z_{\text {ZearaStar }}{ }^{\circledR}\right.$ ) was more convenient to reduce matrix effects than simple SPE clean-up (Bond Elut Mycotoxin). Utilization of a novel clean-up method, the dynamic covalent hydrazine chemistry (DCHC), as described by Siegel et al. (2010), might also be useful in combination with the described ZEN bioassay and should be checked in future.

Apart from the semi-quantitative approach, we successfully applied the described bioassay for a qualitative discrimination of ZEN-producing fungi from others (ZEN was extracted from fungal cultures, mainly Fusarium spp., on rice medium). Compared to the aforementioned procedure for quantification, a simplified sample extraction and assay setup were sufficient to obtain a precise qualitative classification among fungal strains (i.e. pre-incubation of the $z e s 2: \because g f p$ strain could be omitted). Interestingly, we observed a strong correlation between fluorescence response of the G. roseum zes2::gfp bioassay and ZEN concentration in extracts from rice cultures determined by HPLC-MS $(r=0.93)$.

This is the first report of a bioassay that selectively corresponds to ZEN and its reductive metabolite $\alpha$-ZOL, because all known bioassays, advertised for the recognition of ZEN, respond to a broad spectrum of estrogenic compounds, like genistein, $17 \beta$-estradiol and other hormonal substances (Winter et al., 2008; Bovee et al., 2004; Mitterbauer et al., 2003; Mayr et al., 1992; Welshons et al., 1990).

The described zearalenone bioassay can be accomplished with common laboratory equipment and consumables. Compared to liquid chromatography the G. roseum ZEN bioassay is cheap, so it might be suitable for the application in developing countries, but also for nongovernmental organizations, who cannot afford expensive bench-top instruments. Under the condition that the heating can be switched off, the use of a conventional real-time PCR cycler, instead of a fluorescence microplate reader, is possible (Utermark \& Karlovsky, 2006).

The Gliocladium bioassay for ZEN, as described in the present study, could serve as a model approach for a detection of metabolites in biological systems by specific microbial recognition. Nevertheless, this concept requires a broad knowledge about biological interactions and, especially, the molecular level (i.e. identification of a target MO that specifically corresponds to a certain compound, decomposition of the analyte, expressed genes). 
As a whole, this is the first work emphasizing the use of a specific set of volatile biomarkers for the identification of Fusarium contamination in maize and wheat ears that was obtained under seminatural conditions (i.e. in the greenhouse). Volatile markers appeared early and the combination of VOCs allowed differentiating between distinct Fusarium species in maize. Furthermore, we reported for the first time a specific bioassay for the estrogenic Fusarium mycotoxin ZEN. The semi-quantitative assay is applicable for a high throughput-screening of field samples and selectively corresponds to ZEN and its reductive metabolite $\alpha$-ZOL, but no other estrogenic compounds.

Both methods, the volatile biomarker approach and the ZEN-bioassay, could serve as tools for the control of Fusarium and Fusarium related hazards to humans and animals (i.e. through precise sorting of agricultural commodities or target-oriented application of fungicides against Fusarium).

\section{References}

Ai J (1997) Solid Phase Microextraction for Quantitative Analysis in Nonequilibrium Situations. Analytical Chemistry 69:12301236.

Banerjee D, Strobel GA, Booth E, et al. (2010) An endophytic Myrothecium inundatum producing volatile organic compounds. Mycosphere 1:229-240.

Baratta MT, Dorman, HJD, Deans SG, et al. (1998) Antimicrobial and antioxidant properties of some commercial essential oils. Flavour and Fragrance Journal. 13:235-244.

Berna AZ, Lammertyn J, Saevels S, et al. (2004) Electronic nose systems to study shelf life and cultivar effect on tomato aroma profile. Sensors and Actuators B: Chemical 97:324-333.

Bernasconi ML, Turlings TCJ, Ambrosetti L, et al. (1998) Herbivore-induced emissions of maize volatiles repel the corn leaf aphid, Rhopalosiphum maidis. Entomologia Experimentalis et Applicata 87:133-142.

Birkett MA, Bruce TJA, Martin JL, et al. (2004) Responses of female orange wheat Blossom midge, Sitodiplosis mosellana, to wheat panicle volatiles. Journal of Chemical Ecology 30:1319-1328.
Blée E (2002) Impact of phyto-oxylipins in plant defense. Trends in Plant Science 7:315322 .

Borsdorf H, Eiceman GA (2006) Ion Mobility Spectrometry: Principles and Applications. Applied Spectroscopy Reviews 41:323375.

Bottalico A, Perrone G (2002) Toxigenic Fusarium species and mycotoxins associated with head blight in small-grain cereals in Europe. European Journal of Plant Pathology 108:611-624.

Bovee TFH, Helsdingen RJR, Rietjens IMCM, et al. (2004) Rapid yeast estrogen bioassays stably expressing human estrogen receptors $\alpha$ and $\beta$, and green fluorescent protein: a comparison of different compounds with both receptor types. The Journal of Steroid Biochemistry and Molecular Biology 91:99-109.

Brancaleoni E, Scovaventi M, Frattoni M, et al. (1999) Novel family of multi-layer cartridges filled with a new carbon adsorbent for the quantitative determination of volatile organic compounds in the atmosphere. Journal of Chromatography A 845:317-328. 
Cakir A, Kordali S, Kilic H, Kaya E (2005) Antifungal properties of essential oil and crude extracts of Hypericum linarioides Bosse. Biochemical Systematics and Ecology 33:245-256.

Chen X, Zhang Y, Zu Y, Yang L (2012) Chemical composition and antioxidant activity of the essential oil of Schisandra chinensis fruits. Natural Product Research 26:842-849.

Chitarra GS, Abee T, Rombouts FM, Dijksterhuis J (2005) 1-Octen-3-ol inhibits conidia germination of Penicillium paneum despite of mild effects on membrane permeability, respiration, intracellular $\mathrm{pH}$, and changes the protein composition. FEMS Microbiology Ecology 54:67-75.

Combet E, Henderson J, Eastwood DC, Burton KS (2006) Eight-carbon volatiles in mushrooms and fungi: properties, analysis, and biosynthesis. Mycoscience 47:317326.

Cooney JM, Lauren DR, Di Menna ME (2001) Impact of competitive fungi on Trichothecene production by Fusarium graminearum. Journal of Agricultural and Food Chemistry 49:522-526.

Cramer A-CJ, Mattinson DS, Fellman JK, Baik BK (2005) Analysis of Volatile Compounds from Various Types of Barley Cultivars. Journal of Agricultural and Food Chemistry 53:7526-7531.

Daferera DJ, Ziogas BN, Polissiou MG (2000) GCMS Analysis of Essential Oils from Some Greek Aromatic Plants and Their Fungitoxicity on Penicillium digitatum. Journal of Agricultural and Food Chemistry. 48:2576-2581.

Demyttenaere JC., Moriña RM, De Kimpe N, Sandra P (2004) Use of headspace solidphase microextraction and headspace sorptive extraction for the detection of the volatile metabolites produced by toxigenic Fusarium species. Journal of Chromatography A 1027:147-154.

Demyttenaere JCR, Moriña RM, Sandra P (2003) Monitoring and fast detection of mycotoxin-producing fungi based on headspace solid-phase microextraction and headspace sorptive extraction of the volatile metabolites. Journal of Chromatography A 985:127-135.

Desjardins AE, Hohn TM (1997) Mycotoxins in Plant Pathogenesis. Molecular PlantMicrobe Interactions 10:147-152.
Dicke M, Vanbeek TA, Posthumus MA, et al. (1990) Isolation and identification of volatile kairomone that affects acarine predatorprey interactions Involvement of host plant in its production. Journal of Chemical Ecology. 16:381-396.

Dorn B, Forrer H-R, Schuerch S, Vogelgsang S (2009) Fusarium species complex on maize in Switzerland: occurrence, prevalence, impact and mycotoxins in commercial hybrids under natural infection. European Journal of Plant Pathology 125:51-61.

Duckham SC, Dodson AT, Bakker J, Ames JM (2002) Effect of cultivar and storage time on the volatile flavor components of baked potato. Journal of Agricultural and Food Chemistry 50:5640-5648.

Dudareva N, Pichersky E, Gershenzon J (2004) Biochemistry of plant volatiles. Plant Physiology 135:1893-1902.

Eckardt NA (2008) Oxylipin signaling in plant stress responses. Plant Cell 20:495-497.

Falasconi M, Gobbi E, Pardo M, et al. (2005) Detection of toxigenic strains of Fusarium verticillioides in corn by electronic olfactory system. Sensors and Actuators B: Chemical 108:250-257.

Farmer EE, Alméras E, Krishnamurthy V (2003) Jasmonates and related oxylipins in plant responses to pathogenesis and herbivory. Current Opinion in Plant Biology 6:372378.

Girotti JR, Malbrán I, Lori GA, Juárez MP (2012) Early detection of toxigenic Fusarium graminearum in wheat. World Mycotoxin Journal. 5:143-152.

Gobbi E, Falasconi M, Torelli E, Sberveglieri G (2011) Electronic nose predicts high and low fumonisin contamination in maize cultures. Food Research International 44:992-999.

Goebel C, Feussner I (2009) Methods for the analysis of oxylipins in plants. Phytochemistry 70:1485-1503.

Hatanaka A (1993) The biogeneration of green odour by green leaves. Phytochemistry 34:1201-1218.

Henriksen B, Elen O (2005) Natural Fusarium Grain Infection Level in Wheat, Barley and Oat after Early Application of Fungicides and Herbicides. Journal of Phytopathology 153:214-220. 
Hiltpold I, Turlings TCJ (2008) Belowground Chemical Signaling in Maize: When Simplicity Rhymes with Efficiency. Journal of Chemical Ecology 34:628-635.

Holopainen JK, Gershenzon J (2010) Multiple stress factors and the emission of plant VOCs. Trends in plant science 15:176184.

Hosni K, Msaâda K, Ben Taârit M, et al. (2011) Volatile constituents of two Hypericum species from Tunisia. Natural Product Communications 6:1731-1734.

Howe GA, Schilmiller AL (2002) Oxylipin metabolism in response to stress. Current Opinion in Plant Biology 5:230-236.

Huffaker A, Kaplan F, Vaughan MM, et al. (2011) Novel acidic sesquiterpenoids constitute a dominant class of pathogen-induced phytoalexins in maize. Plant physiology 156:2082-2097.

Jeleń HH (2003) Use of solid phase microextraction (SPME) for profiling fungal volatile metabolites. Letters in Applied Microbiology 36:263-267.

Jeleń HH, Majcher M, Zawirska-Wojtasiak R, et al. (2003) Determination of geosmin, 2methylisoborneol, and a musty-earthy odor in wheat grain by SPME-GC-MS, profiling volatiles, and sensory analysis. Journal of Agricultural and Food Chemistry 51:7079-7085.

Jeleń HH, Latus-Ziętkiewicz D, Wąsowicz E, Kamiński E (1997) Trichodiene as a volatile marker for trichothecenes biosynthesis. Journal of microbiological methods 31:45-49.

Joshi RK (2013) Chemical constituents and antibacterial property of the essential oil of the roots of Cyathocline purpurea. Journal of Ethnopharmacology 145:621-625.

Kaminśki E, Stawicki S, Wąsowicz E (1974) Volatile flavor compounds produced by molds of Aspergillus, Penicillium, and Fungi imperfecti. Applied Microbiology 27:1001-1004.

Kamiński E, Libbey LM, Stawicki S, Wąsowicz E (1972) Identification of the predominant volatile compounds produced by Aspergillus flavus. Applied Microbiology 24:721-726.

Keshri G, Magan N, Voysey P (1998) Use of an electronic nose for the early detection and differentiation between spoilage fungi. Letters in Applied Microbiology 27:261264.
Kesselmeier J, Staudt M (1999) Biogenic volatile organic compounds (VOC): an overview on emission, physiology and ecology. Journal of atmospheric chemistry. 33:2388.

Kessler A, Baldwin IT (2001) Defensive Function of Herbivore-Induced Plant Volatile Emissions in Nature. Science. 291:21412144.

Kolakowski BM, Mester Z (2007) Review of applications of high-field asymmetric waveform ion mobility spectrometry (FAIMS) and differential mobility spectrometry (DMS). The Analyst 132:842.

Lin J, Dou J, Xu J, Aisa HA (2012) Chemical Composition, Antimicrobial and Antitumor Activities of the Essential Oils and Crude Extracts of Euphorbia macrorrhiza. Molecules 17:5030-5039.

Liu J, Li L, Ning Z, et al. (2008) Multi-layer Cartridges Filled with Multi-Walled Carbon Nanotubes for the Determination of Volatile Organic Compounds in Indoor Air. Analytical Sciences 24:515-519.

Logrieco A, Mulé G, Moretti A, Bottalico A (2002) Toxigenic Fusarium species and mycotoxins associated with maize ear rot in Europe. European Journal of Plant Pathology 108:597-609.

Magan N, Evans P (2000) Volatiles as an indicator of fungal activity and differentiation between species, and the potential use of electronic nose technology for early detection of grain spoilage. Journal of Stored Products Research 36:319-340.

Matich AJ, Rowan DD, Banks NH (1996) Solid Phase Microextraction for Quantitative Headspace Sampling of Apple Volatiles. Analytical Chemistry 68:4114-4118.

Mayr U, Butsch A, Schneider S (1992) Validation of two in vitro test systems for estrogenic activities with zearalenone, phytoestrogens and cereal extracts. Toxicology 74:135149.

Miedaner T (1997) Breeding wheat and rye for resistance to Fusarium diseases. Plant Breeding 116:201-220.

Mitterbauer R, Weindorfer $\mathrm{H}$, Safaie $\mathrm{N}$, et al. (2003) A Sensitive and inexpensive yeast bioassay for the mycotoxin zearalenone and other compounds with estrogenic activity. Applied and Environmental Microbiology 69:805-811. 
Momin RA, Ramsewak RS, Nair MG (2000) Bioactive compounds and 1,3-di[(cis)-9 octadecenoyl]-2-[(cis, cis ) $-9,12$ octadecadienoyl]glycerol from Apium graveolens L. seeds. Journal of Agricultural and Food Chemistry 48:3785-3788.

Osborne LE, Stein JM (2007) Epidemiology of Fusarium head blight on small-grain cereals. International Journal of Food Microbiology 119:103-108.

Ouyang G, Pawliszyn J (2008) A critical review in calibration methods for solid-phase microextraction. Analytica chimica acta 627:184-197.

Parry DW, Jenkinson P, McLeod L (1995) Fusarium ear blight (scab) in small grain cereals - a review. Plant Pathology 44:207-238.

Perkowski J, Buśko M, Chmielewski J, et al. (2008) Content of trichodiene and analysis of fungal volatiles (electronic nose) in wheat and triticale grain naturally infected and inoculated with Fusarium culmorum. International Journal of Food Microbiology 126:127-134.

Piesik D, Lemńczyk G, Skoczek A, et al. (2011) Fusarium infection in maize: Volatile induction of infected and neighboring uninfected plants has the potential to attract a pest cereal leaf beetle, Oulema melanopus. Journal of Plant Physiology 168:1534-1542.

Placinta C., D’Mello JP., Macdonald AM. (1999) A review of worldwide contamination of cereal grains and animal feed with Fusarium mycotoxins. Animal Feed Science and Technology 78:21-37.

Polizzi V, Adams A, De Saeger S, et al. (2012) Influence of various growth parameters on fungal growth and volatile metabolite production by indoor molds. The Science of the Total Environment 414:277-286.

Porter NG, Wilkins AL (1999) Chemical, physical and antimicrobial properties of essential oils of Leptospermum scoparium and Kunzea ericoides. Phytochemistry 50:407415.

Prost I, Dhont S, Rothe G, et al. (2005) Evaluation of the antimicrobial activities of plant oxylipins supports their involvement in defense against pathogens. Plant Physiology 139:1902-1913.
Rao LJM, Nagalakshimi S, Shankaracharya NB (2000) Studies on chemical and technological aspects of celery (Apium graveolens. Linn) seed. Journal of Food Science and Technology 37:631-635.

Rostás M, Ton J, Mauch-Mani B, Turlings TCJ (2006) Fungal infection reduces herbivoreinduced plant volatiles of maize but does not affect naive parasitoids. Journal of Chemical Ecology 32:1897-1909.

Sabater-Jara AB, Almagro L, Belchí-Navarro S, et al. (2010) Induction of sesquiterpenes, phytoesterols and extracellular pathogenesis-related proteins in elicited cell cultures of Capsicum annuum. Journal of Plant Physiology 167:1273-1281.

Schnee C, Koellner TG, Held M, et al. (2006) The products of a single maize sesquiterpene synthase form a volatile defense signal that attracts natural enemies of maize herbivores. Proceedings of the National Academy of Sciences 103:1129-1134.

Schnuerer J, Olsson J, Boerjesson T (1999) Fungal Volatiles as Indicators of Food and Feeds Spoilage. Fungal Genetics and Biology 27:209-217.

Siegel D, Andrae K, Proske M, et al. (2010) Dynamic covalent hydrazine chemistry as a selective extraction and cleanup technique for the quantification of the Fusarium mycotoxin zearalenone in edible oils. Journal of Chromatography A 1217:2206-2215.

Singh AK, Chanotiya CS, Yadav A, Kalra A (2010) Volatiles of Callicarpa macrophylla: a rich source of selinene isomers. Natural Product Communications 5:269-272.

Smith WEC, Shivaji R, Williams WP, et al. (2012) A maize line resistant to herbivory constitutively releases (E) -betacaryophyllene. Journal of Economic Entomology 105:120-128.

Soković M, Stojković D, Glamočlija J, et al. (2009) Susceptibility of pathogenic bacteria and fungi to essential oils of wild Daucus carota. Pharmaceutical Biology 47:38-43.

Soliman K., Badeaa R. (2002) Effect of oil extracted from some medicinal plants on different mycotoxigenic fungi. Food and Chemical Toxicology 40:1669-1675.

Stenglein SA (2009) Fusarium poae: a pathogen that needs more attention. Journal of Plant Pathology 91:25-36. 
Sunesson A, Vaes W, Nilsson C, et al. (1995) Identification of volatile metabolites from five fungal species cultivated on two media. Applied and Environmental Microbiology 61:2911-2918.

Turlings TCJ, Tumlinson JH, Lewis WJ (1990) Exploitation of Herbivore-Induced Plant Odors by Host-Seeking Parasitic Wasps. Science 250:1251-1253.

Utermark J, Karlovsky P (2006) Quantification of green fluorescent protein fluorescence using real-time PCR thermal cycler. BioTechniques 41:150-154.

Vas G, Vékey K (2004) Solid-phase microextraction: a powerful sample preparation tool prior to mass spectrometric analysis. Journal of Mass Spectrometry 39:233-254.

Vilanova M, Campo E, Escudero A, et al. (2012) Volatile composition and sensory properties of Vitis vinifera red cultivars from north west Spain: correlation between sensory and instrumental analysis. Analytica Vhimica Acta 720:104-111.

Vukovic N, Milosevic T, Sukdolak S, Solujic S (2007) Antimicrobial activities of essential Oil and methanol extract of Teucrium montanum. Evidence-Based Complementary and Alternative Medicine 4:17-20.

Welshons WV, Rottinghaus GE, Nonneman DJ, et al. (1990) A sensitive bioassay for detection of dietary estrogens in animal feeds. Journal of Veterinary Diagnostic Investigation 2:268-273.

Winter P, Nau H, Lampen A, Kamphues J (2008) Detection of estrogenically active substances in diets for sows by an in vitro bioassay supported by HPLC analysis. Journal of Animal Physiology and Animal Nutrition 92:337-344.

Xu X, Nicholson P, Ritieni A (2007) Effects of fungal interactions among Fusarium head blight pathogens on disease development and mycotoxin accumulation. International Journal of food Microbiology 119:67-71. 


\section{Summary}

Fusarium diseases occur worldwide in important agricultural crops and lead to the reduction of yield, seed quality and contamination of food commodities with toxic secondary metabolites, so called mycotoxins. The Fusarium head blight (FHB) of wheat and other smallgrain cereals and the Fusarium ear rot of maize are of particular importance.

In the present work, the use of volatile organic compounds (VOCs) for the detection of Fusarium infections of summer wheat and hybrid maize ears was investigated. Experiments were performed in the greenhouse. Maize ears were infected with $F$. graminearum, $F$. verticillioides and F. subglutinans. Wheat ears were infected with F. graminearum, $F$. avenaceum and $F$. poae. Apart from single infections, also pairwise mixed infections were conducted. A static procedure (solid-phase microextraction, SPME) and a dynamic procedure (open-loop-stripping, OLS) were used for the collection of volatiles. Analysis was performed by GC-MS. A non-parametric test (Kruskal-Wallis) was employed to identify specific volatile biomarkers for infection. A set of 27 volatile markers for Fusarium infection was found in maize. In this case, the combination of VOCs allowed to distinguish between $F$. graminearum and F. verticillioides infection. Wheat trials resulted in a set of 13 characteristic VOCs for Fusarium infection. The selected markers included common 5 to 8 carbon containing volatiles $\left(\mathrm{C}_{5}-\mathrm{C}_{8}\right)$ being widespread among plants and microbes, but also infection-specific sesquiterpenoid markers $\left(\mathrm{C}_{15} \mathrm{H}_{24}\right)$. Monitoring of the temporal VOCs emission revealed that volatile markers were largely present at early time points. Thus, in maize the detection was possible before symptoms were visible ( $4-8 \mathrm{dpi})$. Monitoring of volatile profiles with respect to marker compounds, i.e. with portable detectors in the field or in storage facilities, offers the possibility for a fast and non-destructive detection of Fusarium infections and concurrently the prediction of mycotoxin contamination.

The macrocyclic acid lactone zearalenone (ZEN), produced by several Fusarium spp., exerts estrogenic effects in animals and humans and occurs frequently in feed and foodstuff in the temperate regions. Especially pigs are highly susceptible. So far, several bioassays have been described for the detection of ZEN. The available assays are largely based on the human 
estrogen receptors $\alpha$ and $\beta$ and do not selectively correspond to ZEN, but to a broad spectrum of estrogens (i.e. genistein, 17ß-estradiol). This work is the first report of a specific bioassay for ZEN and its most critical metabolite $\alpha$-zearalenol $(\alpha-\mathrm{ZOL})$. The assay is based on a recombinant strain of the mycoparasite Gliocladium roseum (zes2::gfp) and allows a ZEN detection from field samples (i.e. contaminated maize). The sample preparation, including immunoaffinity clean-up, and conditioning of the indicator strain (i.e. pre-cultivation) were optimized. The assay was suitable for a qualitative detection of ZEN in a broad concentration range and for a quantitative evaluation of ZEN contamination in maize field samples in the range of $0.9 \mathrm{mg} \mathrm{kg}^{-1}$ to $90 \mathrm{mg} \mathrm{kg}^{-1}$. Furthermore, the assay was successfully applied for a qualitative fungal strain screening for ZEN production. The G. roseum zes $2: \because g f p$ bioassay can be accomplished with common laboratory equipment and might be suitable for the application in developing countries. 


\section{Zusammenfassung}

Phytopathogene Fusarium spp. treten weltweit in landwirtschaftlichen Kulturen auf und führen häufig zur Ertragsreduktion, Verschlechterung der Produktqualität sowie Kontaminationen der Erntegüter mit toxischen Sekundärmetaboliten, sog. Mykotoxinen. Die durch Fusarium spp. hervorgerufene partielle Taubährigkeit (FHB) des Weizens und anderer Getreidearten sowie die Fusarium Kolbenfäule an Mais sind aus ökonomischer Sicht von besonderer Bedeutung.

Im Rahmen dieser Arbeit wurde die Verwendung von volatilen organischen Verbindungen (VOCs) zur Detektion von Fusariosen an Sommerweizen und Hybridmais unter Gewächshausbedingungen untersucht. Maiskolben wurden mit F.graminearum, F. verticillioides und F. subglutinans infiziert, während Weizenähren mit Sporensuspensionen von $F$. graminearum, $F$. avenaceum und $F$. poae inokuliert wurden. Auch Mischinfektionen wurden durchgeführt. Für die Sammlung der VOCs wurde ein statisches Verfahren (Festphasenmikroextraktion, SPME) sowie ein dynamisches Verfahren (open-loop-stripping, OLS) eingesetzt. Die Analyse erfolgte in beiden Fällen mittels GC-MS. Ein nichtparametrischer Test (Kruskal-Wallis) wurde zur Identifikation von spezifischen volatilen Markern herangezogen. Auf diese Weise konnte an Mais ein Set aus 27 volatilen Biomarkern für die Infektion mit Fusarium spp. ermittelt werden. Die Kombination der VOCs ermöglichte hier die Unterscheidung zwischen Infektionen mit $F$. graminearum und $F$. verticillioides. An Weizen konnte ein Set aus 13 charakteristischen VOCs für den Fusarium Befall ermittelt werden. Die selektierten volatilen Marker beinhalteten sowohl einfache Moleküle mit 5 bis 8 Kohlenstoffatomen $\left(\mathrm{C}_{5}-\mathrm{C}_{8}\right)$, welche häufig von Pflanzen und Mikroorganismen emittiert werden, als auch infektionsspezifische Sesquiterpene $\left(\mathrm{C}_{15} \mathrm{H}_{24}\right)$. In Zeitreihenversuchen konnte gezeigt werden, dass ein Großteil der relevanten VOCs bereits nach kurzer Zeit emittiert wird. So waren in Mais volatile Biomarker detektierbar, bevor Symptome am Kolben erkennbar waren (4 - 8 Tage nach der Inokulation). Ein Monitoring von VOC-Profilen im Hinblick auf volatile Marker könnte eine schnelle und nicht-destruktive Detektion von Fusarium Infektionen (ggf. auch Risikoabschätzung zur Mykotoxinbelastung), z.B. im Feld oder Lager, ermöglichen. Hierfür stehen transportable Detektoren zur Verfügung. 
Das makrozyklische Lacton Zearalenon (ZEN) wird von mehreren Fusarium spp. produziert und besitzt eine östrogene Wirkung auf den menschlichen und tierischen Organismus. Schweine gelten diesbezüglich als besonders anfällig. ZEN wird in gemäßigten Klimazonen regelmäßig in Lebens- und Futtermitteln nachgewiesen. Bislang wurden zahlreiche Bioassays für die Detektion von ZEN beschrieben. Sie basieren meist auf den menschlichen Östrogenrezeptoren $\alpha$ und $\beta$ und reagieren unspezifisch auf eine Vielzahl von östrogenen Substanzen (z.B. Genistein, 17ß-Estradiol). Die vorliegende Arbeit beschreibt erstmalig ein Bioassay zur spezifischen Detektion von ZEN sowie dem kritischen Metabolit $\alpha$-Zearalenol $(\alpha-Z O L)$. Das Assay basiert auf einer zes2::gfp Mutante des Mykoparasiten Gliocladium roseum und ermöglicht eine Detektion von ZEN in Feldproben (z.B. kontaminierter Mais). Schritte zur Probenvorbereitung und Extraktion, einschließlich Aufreinigung mit Immunoaffinitätssäulen, sowie die Kultur des Inditaktorstammes wurden optimiert. Das Assay eignet sich für die qualitative Detektion von ZEN in einem weiten Konzentrationsbereich sowie für eine quantitative ZEN Bestimmung in kontaminierten Mais Feldproben im Bereich zwischen $0,9 \mathrm{mg} \mathrm{kg}^{-1}$ und $90 \mathrm{mg} \mathrm{kg}^{-1}$. Neben der Detektion in Feldproben, konnte das Bioassay erfolgreich für ein Screening von Pilzstämmen zur Identifikation von ZEN-Produzenten eingesetzt werden. Das hier beschriebene G. roseum zes2:gfp Bioassay kann mit einer einfachen Laborausstattung durchgeführt werden und eignet sich möglicherweise für die Anwendung in Entwicklungsländern. 


\section{Acknowledgements}

First of all, I would like to acknowledge my supervisor Prof. Dr. Petr Karlovsky, who provided the subject of this work and supported me throughout the course of the thesis with advice and encouragement. I thank him for the motivation and good discussion during the last three years.

Furthermore, I acknowledge the committee members Prof. Dr. Stefan Schuetz, as second examiner, and Prof. Dr. Andreas von Tiedemann.

My thank goes to the Federal Ministry of Education and Research for the funding of the project 'MycoSensExpert' and the German DLR for administrative support.

I would like to express my deepest thank to Dr. Richard Splivallo for his help, guidance and encouragement from the very beginning of my work in the lab. He put great effort into instructing me about VOC sampling and analysis. He always had an open ear, even during times, when a GC-MS trouble-shooting was the last thing we needed. Apart from this, I thank him very much for the check of my thesis.

I acknowledge Dr. Cornelia Herrfurth and her team (group leader: Prof. Dr. Ivo Feußner, Albrecht-von-Haller-Institute for Plant Sciences) for the helpful advice during GC-MS measurements and Dr. Tobias Koellner and Sandra Irmisch (Max Planck Institute for Chemical Ecology) for the opportunity to analyse my samples in Jena.

I am thankful to all members of the Karlovsky lab, in particular Heike Rollwage, Ruth Pilot, Nadine Reimann and Patricia Bartoschek, for their advice and technical support, especially during the first months. I thank our apprentices Sarah Heppner, Jacqueline Moser and Magdalena Boemeke for their technical assistance. I thank my colleagues Dr. Sabine Nutz for introducing me into the secrets of real-time PCR and Dr. Sasithorn Limsuwan (Joy), Katharina Doell and Dr. Philip Koessler for their support with toxin extraction and HPLC-MS measurements. I thank our $\mathrm{PhD}$ students and colleagues from abroad for all the culinary delights, especially the Chatterjees for demonstrating how spicy a meal could be, Husam for 
self-made hummus and Yi for sushi and some tasty snack that I cannot name anymore. It was a pleasure to meet you all and I wish you all the best for your future.

My sincere thanks go to the whole division for the good working atmosphere and support, especially to Mrs. Kistner for administrative support and patience and Mr. Hodyl for attending my plants so well.

I am thankful to my family for the support and motivation during the last years and my friends for all the fun, good discussion and opportunity to disconnect.

My deepest thank goes to my husband Johannes, just for being there. 


\section{Curriculum vitae}

Personal Information

Name:

E-mail:

\section{Work Experience}

Since Jan 2013

\section{Academic Education}

Oct 2009 - May 2013

Oct 2007 - Sep 2009

Oct 2005 - Jul 2007

Oct $2004-$ Sep 2005

\section{School}

Aug 1995 - Jun 2004

Aug 1991 - Jul 1995
Eva-Maria Becker (nee Kuhl)

evambec@gmail.com

DHD-Consulting GmbH, Hildesheim, Germany

$\mathrm{Ph} . \mathrm{D}$ program for Agricultural Science, Molecular Phytopathology and Mycotoxin Research Unit, Division of Plant Pathology and Plant Protection, Georg August University Goettingen, Germany

Master program, Agricultural sciences with emphasis on plant production, Georg August University Goettingen, Germany

Master thesis: 'Distribution of plant functional traits in a range of grassland formations differing in water and nitrogen availability - A comparison using the SynBioSys and BiolFlor databases' (M. Sc. with distinction)

Continuation of Bachelor program, Agricultural sciences with emphasis on plant production, Georg August University Goettingen, Germany

Agricultural sciences, Justus Liebig University Giessen, Germany

Grammar school

Elementary school 


\section{Eidesstattliche Erklärungen}

1. Hiermit erkläre ich, dass diese Arbeit weder in gleicher noch in ähnlicher Form bereits anderen Prüfungsbehörden vorgelegen hat.

Weiter erkläre ich, dass ich mich an keiner anderen Hochschule um einen Doktorgrad beworben habe.

Göttingen, den

(Unterschrift)

2. Hiermit erkläre ich eidesstattlich, dass diese Dissertation selbständig und ohne unerlaubte Hilfe angefertigt wurde.

Göttingen, den

(Unterschrift) 